UNIVERSITAT POLITĖCNICA DE VALĖNCIA

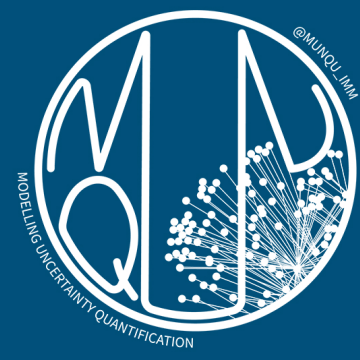

Advances on Uncertainty Quantification Techniques for Dynamical Systems: Theory and Modelling

$\mathrm{PhD}$. Thesis

PhD. Candidate

Clara Burgos Simón
Supervisors

Dr. Juan Carlos Cortés López Dr. Rafael Jacinto Villanueva Micó 



\section{Advances on Uncertainty Quantification Techniques for Dynamical Systems: Theory and Modelling}

Author: Clara Burgos Simón

Supervisors: Dr. Juan Carlos Cortés López

Dr. Rafael Jacinto Villanueva Micó 



\section{Declaration of Authorship}

Dr. Juan Carlos Cortés López and Rafael Jacinto Villanueva Micó, full professors at the Universitat Politècnica de València,

CERTIFY that the present PhD thesis entitled: Advances on Uncertainty Quantification Techniques for Dynamical Systems: Theory and Modelling has been performed under our supervision at the Instituto de Matemática Multidisciplinar in the Universitat Politècnica de València by Clara Burgos Simón. It constitutes her thesis dissertation to obtain the $\mathrm{PhD}$ degree in Mathematics with international mention.

In compliance with the current legislation, we authorize the presentation of this dissertation signing the present certificate.

Valencia - March 2021

Dr. Juan Carlos

Cortés López
Dr. Rafael Jacinto

Villanueva Micó 
Creativity is the ability to introduce order into the randomness of nature. 
A mis padres.

A mi hermana.

A mi número uno... 

La cuantificación de la incertidumbre está compuesta por una serie de métodos y técnicas computacionales cuyo objetivo principal es describir la aleatoriedad presente en problemas de diversa índole. Estos métodos son de utilidad en la modelización de procesos biológicos, físicos, naturales o sociales, ya que en ellos aparecen ciertos aspectos que no pueden ser determinados de manera exacta. Por ejemplo, la tasa de contagio de una enfermedad epidemiológica o el factor de crecimiento de un volumen tumoral dependen de factores genéticos, ambientales o conductuales. Estos no siempre pueden definirse en su totalidad y por tanto conllevan una aleatoriedad intrínseca que afecta en el desarrollo final. El objetivo principal de esta tesis es extender técnicas para cuantificar la incertidumbre en dos áreas de las matemáticas: el cálculo de ecuaciones diferenciales fraccionarias y la modelización matemática.

Las derivadas de orden fraccionario permiten modelizar comportamientos que las derivadas clásicas no pueden, como por ejemplo los efectos de memoria o la viscoelasticidad en algunos materiales. En esta tesis, desde un punto de vista teórico, se extenderá el cálculo fraccionario a un ambiente de incertidumbre, concretamente en el sentido de la media cuadrática. Se presentarán problemas de valores iniciales fraccionarios aleatorios. El cálculo de la solución, la obtención de las aproximaciones de la media y varianza de la solución y la aproximación de la primera función de densidad de probabilidad de la solución son conceptos que se abordarán en los próximos capítulos. Sin embargo, no siempre es sencillo obtener la solución exacta de un problema de valores iniciales fraccionario aleatorio. Por ello en esta tesis también se dedicará un capítulo para describir un procedimiento numérico que aproxime su solución.

Por otro lado, desde un punto de vista más aplicado, se desarrollan técnicas computacionales para cuantificar la incertidumbre en modelos matemáticos. Combinando estas técnicas junto con modelos matemáticos apropiados, se estudiarán problemas de dinámica biológica. En primer lugar, se determinará la cantidad de portadores de meningococo en España con un modelo de competencia de Lotka-Volterra fraccionario aleatorio. A continuación, el volumen de un tumor ma- 
mario se modelizará mediante un modelo logístico con incertidumbre. Finalmente ayudándonos de un modelo matemático que describe el nivel de glucosa en sangre de un paciente diabético, se pretende dar una recomendación de carbohidratos e insulina que se debe de ingerir para que el nivel de glucosa del paciente esté dentro de una banda de confianza saludable. Es importante subrayar que para poder realizar estos estudios se requieren datos reales, los cuales pueden estar alterados debido a los errores de medición o proceso que se han cometido para obtenerlos. Por este motivo, modelizar correctamente el problema junto con la incertidumbre en los datos es de vital importancia. 


\section{Resum}

La quantificació de la incertesa està composada per una sèrie de mètodes i tècniques computacionals, l'objectiu principal de les quals és descriure l'aleatorietat present en problemes de diversa índole. Aquests mètodes són d'utilitat en la modelització de processos biològics, físics, naturals o socials, ja que en ells apareixen certs aspectes que no poden ser determinats de manera exacta. Per exemple, la taxa de contagi d'una malaltia epidemiològica o el factor de creixement d'un volum tumoral depenen de factors genètics, ambientals o conductuals. Aquests no sempre poden definir-se íntegrament i per tant, comporten una aleatorietat intrínseca que afecta en el desenvolupament final. L'objectiu principal d'aquesta tesi doctoral és estendre tècniques per a quantificar la incertesa en dues àrees de les matemàtiques: el càlcul d'equacions diferencials fraccionàries i la modelització matemàtica.

Les derivades d'ordre fraccionari permeten modelitzar comportaments que les derivades clàssiques no poden, com per exemple, els efectes de memòria o la viscoelasticitat en alguns materials. En aquesta tesi, des d'un punt de vista teòric, s'estendrà el càlcul fraccionari a un ambient d'incertesa, concretament en el sentit de la mitjana quadràtica. Es presentaran problemes de valors inicials fraccionaris aleatoris. El càlcul de la solució, l'obtenció de les aproximacions de la mitjana i, la variància de la solució i l'aproximació de la primera funció de densitat de probabilitat de la solució són conceptes que s'abordaran en els pròxims capítols. No obstant això, no sempre és senzill obtindre la solució exacta d'un problema de valors inicials fraccionari aleatori. Per això en aquesta tesi també es dedicarà un capítol per a descriure un procediment numèric que aproxime la seua solució.

D'altra banda, des d'un punt de vista més aplicat, es desenvolupen tècniques computacionals per a quantificar la incertesa en models matemàtics. Combinant aquestes tècniques juntament amb models matemàtics apropiats, s'estudiaran problemes de dinàmica biològica. En primer lloc, es determinarà la quantitat de portadors de meningococ a Espanya amb un model de competència de LotkaVolterra fraccionari aleatori. A continuació, el volum d'un tumor mamari es modelitzará mitjançant un model logístic amb incertesa. Finalment ajudant-nos d'un model matemàtic que descriu el nivell de glucosa en sang d'un pacient diabètic, 
es pretén donar una recomanació de carbohidrats i insulina que s'ha d'ingerir perquè el nivell de glucosa del pacient estiga dins d'una banda de confiança saludable. És important subratllar que per a poder realitzar aquests estudis es requereixen dades reals, els quals poden estar alterats a causa dels errors de mesurament o per la forma en que s'han obtés. Per aquest motiu, modelitzar correctament el problema juntament amb la incertesa en les dades és de vital importància. 
Uncertainty quantification collects different methods and computational techniques aimed at describing the randomness in real phenomena. These methods are useful in the modelling of different processes as biological, physical, natural or social, since they present some aspects that can not be determined exactly. For example, the contagious rate of a epidemiological disease or the growth factor of a tumour volume depend on genetic, environmental or behavioural factors. They may not always be fully described and therefore involve uncertainties that affects on the final result. The main objective of this $\mathrm{PhD}$ thesis is to extend techniques to quantify the uncertainty in two mathematical areas: fractional calculus and mathematical modelling.

Fractional derivatives allow us to model some behaviours that classical derivatives cannot, such as memory effects or the viscoelasticity of some materials. In this $\mathrm{PhD}$ thesis, from a theoretical point of view, fractional calculus is extended into the random framework, concretely in the mean square sense. Initial value problems will be studied. The calculus of the analytic solution, approximations for the mean and for the variance and the computation of the first probability density function are concepts we deal with them thought the following chapters. Nevertheless, it is not always possible to obtain the analytic solution of an initial value problem. Therefore, in this dissertation a chapter is addressed to describe a numerical procedure to approximate the solution for an initial value problem.

On the other hand, from a modelling point of view, computational techniques to quantify the uncertainty in mathematical models are developed. Merging these techniques with appropriate mathematical models, problems of biological dynamics are studied. Firstly, the carriers of meningococcus in Spain are determined using a competition Lotka-Volterra random fractional model. Then, the volume of breast tumours is modelled by a random logistic model. Finally, taking advantage of a mathematical model which describes the glucose level of a diabetic patient, a recommendation of insulin shots and carbohydrate intakes is proposed to a patient in order to maintain her/his glucose level in a healthy confidence range. An important observation is that to carry out these studies real data is required and 
they may include uncertainties contained in the measurements on the process to perform the corresponding study. This it is the reason why it is crucial to properly model the problem taking also into account the randomness of the data. 


\section{Contents}

Abstract ix

Contents $\quad$ Xv

1 General Introduction 1

2 Riemann-Liouville and Caputo operators in mean square sense: solving a random linear fractional differential equation $\quad 7$

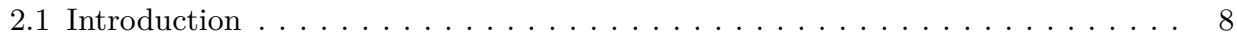

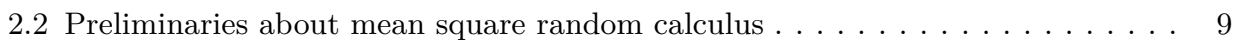

2.3 Mean square random fractional differential and integral operators . . . . . . . . 15

2.4 Solving the random linear fractional differential equation by the mean square

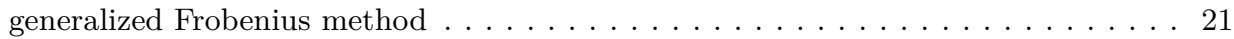

2.5 Computing approximations of the mean, the variance, the covariance and the cross-covariance functions of the solution stochastic process . . . . . . . . . . . 29

2.6 Numerical examples . . . . . . . . . . . . . . . . . . . . . . 33

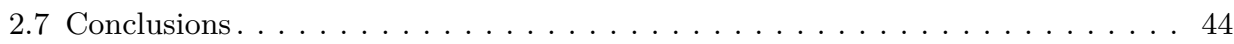

3 Solving high order random mean square fractional linear differential equations 45

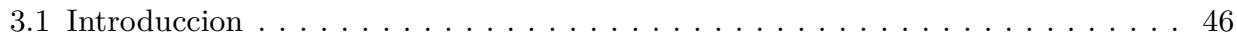

3.2 Introducing a key class of random variables $\ldots \ldots \ldots \ldots \ldots$

3.3 Mean square convergence of the random generalized power series solution . . . 53

3.4 Numerical examples . . . . . . . . . . . . . . . . . . . . . . . 63

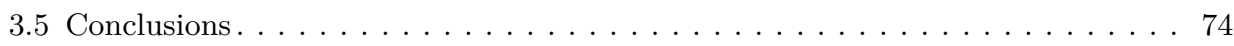


4 A full probabilistic solution of the random linear fractional differential equation

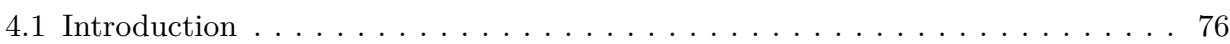

4.2 Main result: Computing approximations of the 1-PDF of the solution stochastic

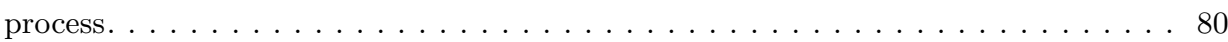

4.3 Numerical examples . . . . . . . . . . . . . . . . . . . . 86

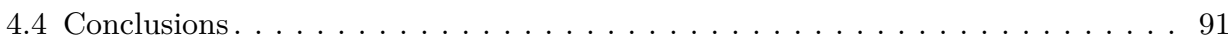

5 Solving a random non-autonomous linear fractional differential equation 93

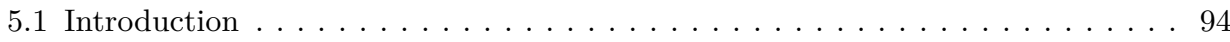

5.2 Constructing a mean square convergent random generalized power series solution and approximating its main statistical properties . . . . . . . . . . . . . 94

5.3 Numerical examples . . . . . . . . . . . . . . . . . . . . . . . . . . . . . 99

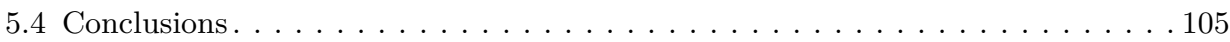

6 Solving high order random non-autonomous linear fractional differential equations: generalizing Airy differential equation 107

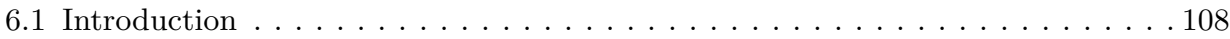

6.2 Constructing the solution stochastic process . . . . . . . . . . . . . . . 109

6.3 Probabilistic properties of the solution stochastic process . . . . . . . . . . 114

6.4 Approximating the probability density function via the Principle of Maximum

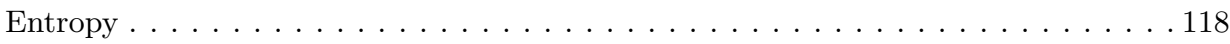

6.5 Numerical examples . . . . . . . . . . . . . . . . . . . . . . . 121

6.6 Conclusions . . . . . . . . . . . . . . . . . . . . . . . . . . . . 134

7 Mean square convergent numerical solutions of random fractional $\begin{array}{ll}\text { differential equations } & 137\end{array}$

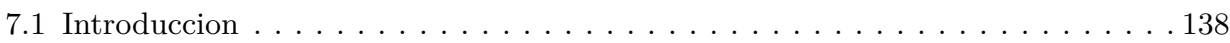


7.2 Relation between the random fractional differential and integral equations . . . 139

7.3 Numerical approximations to the random fractional IVP . . . . . . . . . . . . 143

7.4 Error analysis . . . . . . . . . . . . . . . . . . . . . . . . . . . . . 144

7.5 Statistical moments of the numerical approximations . . . . . . . . . . . . 147

7.6 Numerical examples . . . . . . . . . . . . . . . . . . . . . . . . . . . . . . . 149

7.7 Conclusions. . . . . . . . . . . . . . . . . . . . . . . . . . . . . . 159

8 Studying the evolution over the next few years of the meningococcal genogroup W carriers in Spain 161

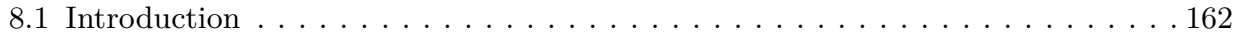

8.2 Lotka-Volterra competition model and SCS epidemilogical transmission . . . . . 165

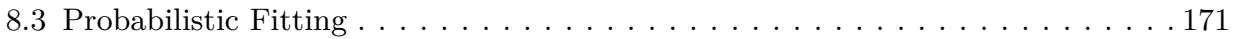

8.4 Results . . . . . . . . . . . . . . . . . . . . . . . . . . . . . . 177

8.5 Conclusions. . . . . . . . . . . . . . . . . . . . . . . . . . . . . . . 182

9 Modelling breast tumor growth by a randomized logistic model 185

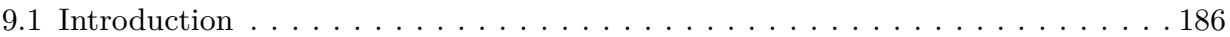

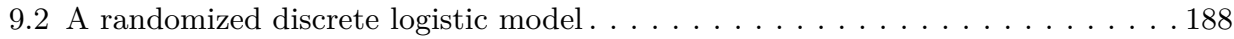

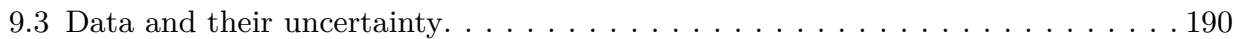

9.4 Statistical distribution of the model parameters. . . . . . . . . . . . . . . . 194

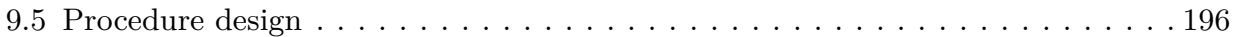

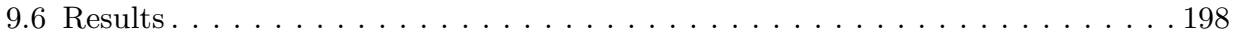

9.7 Conclusions . . . . . . . . . . . . . . . . . . . . . . . . . . . . . 203

10 A random computational procedure to recommend insulin and carbohydrates intakes to diabetic patients 205

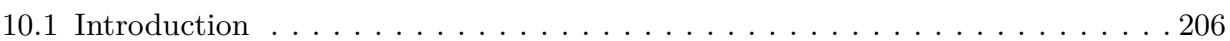

10.2 Model description . . . . . . . . . . . . . . . . . . . . . . . . . . 209 


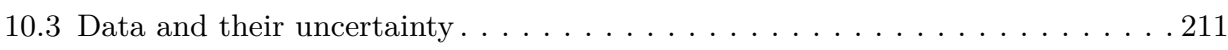

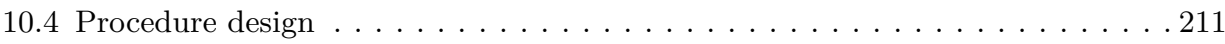

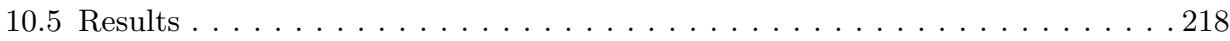

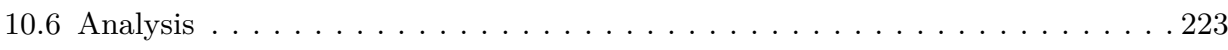

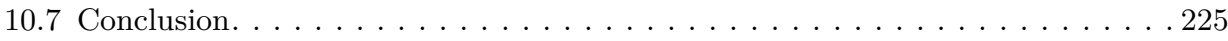

11 General Conclusions $\quad 227$

$\begin{array}{ll}\text { Bibliography } & 229\end{array}$ 


\section{List of Figures}

2.1 Approximations of the mean (top) and the standard deviation (bottom) of the solution SP to the random IVP (2.20) with $\alpha=0.7$ using different orders of truncations $M$ over the time intervals $[0,5]$ and $[0,10]$ in the context of Example 2.2. . . . . . . . 36

2.2 Correlation coefficient function $\rho_{Y_{M}}(t, s)$ of the approximation $Y_{M}(t)$ of order $M=20$ of the solution SP $Y(t)$ to the random IVP (2.20) with $\alpha=0.7$ over the time domain $(t, s) \in[0,5] \times[0,5]$ in the context of Example 2.2. . . . . . . . . . . . . . . 38

2.3 Approximations of the mean (top) and the standard deviation (bottom) of the solution SP to the random IVP (2.20) varying the fractional differentiation parameter $\alpha=\{0.1,0.2, \ldots, 0.9,1\}$ taking as order of truncation $M=20$ over the time interval $[0,5]$ in the context of Example 2.2. . . . . . . . . . . . . . .

3.1 Approximations of the mean (left) and the standard deviation (right) of the solution SP to the random fractional IVP (3.1) with $\alpha=0.3$ (Case I) taking different orders of truncation $M$ over the time interval $[0,5]$ in the context of Example $3.5 \ldots \ldots \ldots$

3.2 Approximations of the mean (left) and the standard deviation (right) of the solution SP to the random fractional IVP (3.1) with $\alpha=0.7$ (Case I) taking different orders of truncation $M$ over the time interval $[0,8]$ in the context of Example 3.5 . . . . . 66

3.3 Approximations of the mean (left) and the standard deviation (right) of the solution SP to the random fractional IVP (3.1) with $\alpha=0.6$ (Case I) taking different orders of truncation $M$ over the time interval $[0,30]$ in the context of Example 3.6. . . . . . . 68 
3.4 Approximations of the mean (left) and the standard deviation (right) of the solution SP to the random fractional IVP (3.1) with $\alpha=0.5$ (Case I) using different orders of truncation $M$ over the time intervals $[0,60]$ and $[0,30]$, respectively in the context of Example 3.6. 70

3.5 Relative errors, given in (3.32) and (3.33), of the approximations of the mean (left) and the standard deviation (right) of the solution SP to the random fractional IVP (3.1) with $\alpha=0.5$ (Case I) using different orders of truncation $M$ over the time intervals $[0,60]$ and $[0,30]$, respectively, in the context of Example 3.6. For the sake of clarity, in both plots, we present a zoom around of the end-points $t=50$ and $t=25$ of the convergence regions for the approximations of the mean and standard deviation, respectively. . . . . . . . . 70

3.6 Approximations of the mean (left) and the standard deviation (right) of the solution SP to the random fractional IVP (3.1) with $\alpha=1.2$ (Case II) using different orders of truncation $M$ over the time intervals $[0,8]$ and $[0,5]$, respectively in the context of Example

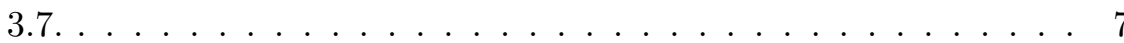

3.7 Approximations of the correlation coefficient associated to the solution SP to the random fractional IVP (3.1) with $\alpha=1.2$ (Case II) taking as order of truncation $M=20$ in the context of Example 3.7. 73

4.1 Plots of the 1-PDF, $f_{1}^{M}(y, t)$, given by (4.14), for different truncations $M$ at the time instants $\hat{t}=0.1$ (left), $\hat{t}=1$ (center) and $\hat{t}=5$ (right) in Example 4.1. . . . . . . . . . . . . . .

4.2 Plots of approximations $\mu_{y^{M}}(t)$ (left) and $\sigma_{y^{M}}^{2}(t)$ (right), given by (4.23) and (4.24), respectively, to the exact mean and variance of the solution SP to random fractional IVP (4.3), respectively, for different values of the truncation order $M$ in the context of Example $4.1 \ldots \ldots \ldots \ldots \ldots \ldots \ldots$ 
4.3 Plots of the 1-PDF, $f_{1}^{M}(y, t)$, given by (4.14), for different truncations $M$ at the time instants $\hat{t}=0.05$ (left), $\hat{t}=0.2$ (centre) and $\hat{t}=1$ (right) in Example 4.2. . . . . . . . . . . . . . .

4.4 Plots of approximations $\mu_{y^{M}}(t)$ (left) and $\sigma_{y^{M}}^{2}(t)$ (right), given by (4.23) and (4.24), respectively, to the exact mean and variance of the solution SP to random fractional IVP (4.3), respectively, for different values of the truncation order $M$ in the context of Example

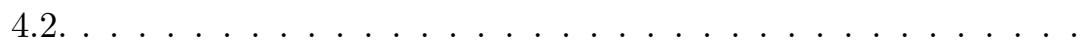

5.1 Approximations of the mean and the standard deviation of the solution SP to the random IVP (5.1) using different orders of truncations $M$ in Case I $(p<\alpha)$ described in the context of Example 6.1. Notice that the approximations corresponding to $M=12$ and $M=14$, for the mean and the standard deviation, match on the whole time interval $t \in[0,15]$, thus showing convergence. . . . . . . 101

5.2 Approximations of the mean and the standard deviation of the solution SP to the random IVP (5.1) using different orders of truncations $M$ in Case II $(p=\alpha)$ described in the context of Example 6.1. On the left side, we have delineated the domain of convergence for the mean and the standard deviation plotting a vertical line. On the right side, we show a zoom on a piece of the domain of convergence, for the sake of clarity. Observe that the approximations corresponding to $M=4,6,8,10$ match for the mean, while this same fact happens when $M=9,10$, in the case of the standard deviation.

6.1 Approximations of the mean $\left(\mathbb{E}\left[Y_{M}(t)\right]\right)$ and the variance $\left(\mathbb{V}\left[Y_{M}(t)\right]\right)$ of the solution SP to the random IVP (6.1) using different orders of truncations $M$ over the interval $t \in[0,5]$ in the context of Example

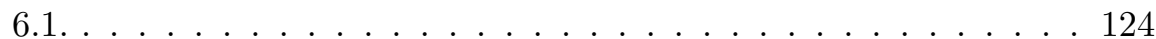


6.2 Approximations of the mean $\left(\mathbb{E}\left[Y_{M}(t)\right]\right)$ and the variance $\left(\mathbb{V}\left[Y_{M}(t)\right]\right)$ of the solution SP to the random IVP (6.1) using different orders of truncations $M$ over the interval $t \in[0,9]$ in the context of Example $6.1 \ldots \ldots \ldots \ldots \ldots \ldots \ldots$

6.3 Approximate PDF, $f_{Y_{M}(t)}(y, t)$, of the solution SP $Y(t)$ to random fractional IVP (6.1) with order of the fractional order $\alpha=1.9$ in the context of Example 6.1 using the PME for the approximate solution SP $Y_{M}(t)$ with $M=30$ and $0 \leq t \leq 9 \ldots \ldots 128$

6.4 Approximations of the mean $\left(\mathbb{E}\left[Y_{M}(t)\right]\right)$ and the standard deviation $\left(\sigma\left[Y_{M}(t)\right]\right)$ of the solution SP to the random IVP (6.1) using different orders of truncations $M$ in the interval $t \in[0,5]$ in the context of Example 6.2. . . . . . . . . . . . . . . . 13

6.5 Surface of the correlation coefficient of the solution SP, defined in (6.27), with $M=6$ over the domain $(t, s) \in[0,5] \times[0,5]$ in the context of Example 6.2. . . . . . . . . . . . . . . 132

7.1 Approximate 1-PDF for the random fractional IVP (7.1) obtained via the combination of the PME and the random numerical scheme. Example 7.1. . . . . . . . . . . . . . . . . . . 153

7.2 Graphical comparison between the exact 1-PDF given in (7.33) and its approximations obtained via PME at different times $t=$ $0.1,0.2,0.3,0.4$. Example 7.1. . . . . . . . . . . . . 155

7.3 Approximate 1-PDF for the IVP (7.34) using PME combined wit the approximations of the mean and the variance obtained via the random fractional numerical scheme. . . . . . . . . . . . . . 159

8.1 Diagram of Susceptible-Carrier-Susceptible (SCS) model in Eq. (8.1).167 
8.2 $95 \%$ CI of the solution SP of the percentage of carriers given by model (8.2). The gray lines represents the percentiles 2.5 and 97.5 and the red one, the mean, for each month, $t$, from Dec 2011 until Dec 2021. . . . . . . . . . . . . . . . . . . 178

8.3 Probability density functions for the parameters $\beta$ (blue line) and $\gamma$ (red line) of model (8.2). Notice that there are non-zero probabilities for negative values of these parameters, although in any reasonable epidemiological model they are considered positive. This feature is a consequence of the kernel technique and these negative unrealistic values can be overlooked. . . . . . . . . . . . . . . 179

8.4 Confidence intervals of the solution SP for the percentage of meningococcus W-135 computed by the numerical scheme given in Eq. (8.13). The gray lines represents the percentiles 2.5 and 97.5 and the red line, the mean, for each time, $t$, from Dec 2011 until Dec 2020. . . 180

8.5 PDFs for the parameters of the Lotka-Volterra model. The red line corresponds to the parameter $N_{1}$ and the blue line to the fractional derivative order, $\alpha$. The short piece of negative values of the $\alpha$ parameter that appears in the plot, is a feature of the application of the so-called kernel technique used to construct the PDFs. This piece must been neglected since $0<\alpha<1$. . . . . . . . . . . . . 181

8.6 Probability of having 5\% of carriers of Men W-135 or more in Spain. Prediction from Dec 2011 until Dec 2021. . . . . . . . . . . . 181

9.1 PDF of each sampled data using the PME at the days $\tilde{n} \in\{0,16,30,33,43,48\}$. The red points represent the values $\tilde{m}_{1, \tilde{n}}$ given in Table 9.1. . . . . 193

9.2 PDFs of random model parameters $A$ and $B$ of the randomized discrete logistic model described by equations (9.10) and (9.11), respectively. .................... 200 
9.3 Comparison between the PDF of each sampled data (red dashed lines) and the PDF of the fitting randomized logistic model (blue lines) at the days $\tilde{n} \in\{0,16,30,33,43,48\}$. In the horizontal axis of each panel, the red point represents the sampled data (it corresponds to column $\tilde{m}_{1, \tilde{n}}$ in Table 9.1) and the blue point represents the mean or expectation obtained via the PDF (blue curves). . . . . . 201

9.4 Representation of the PDF, $f_{X_{n}}$, of the random discrete logistic model (9.6) for different days $n=0,1, \ldots, 50$. Red points represent the sampled values of tumor volume described in the column $\tilde{m}_{1, \tilde{n}}$ of Table 9.1 and green points represent the mean of the distribution defined by the blue curves. . . . . . . . . . . . . . . . . 202

10.1 According to the expression (10.8), we have: (a) $\tau$ is zero if the level of glucose returned by the model, red dot, lie inside the CI95\%, blue dots. (b) $\tau$ is the minimum distance from the level of glucose returned by the model, red dot, and the CI95\%, blue dots, if they are not inside CI95\%. In this case, the distance between the red dot to the Perc 2.5 blue dot. . . . . . . . . . . . . . . . . 213

10.2 Fitness function $F_{E}$ measures the difference between the model output mean and CI95\% (red dashed line and red shadowed band) and the data mean and CI95\% (blue dashed line and blue shadowed band). The selection algorithm find sets of model parameter values in such a way that the red and the blue bands are as much similar as possible. . . . . . . . . . . . . . . 2 215 
10.3 Patient 1. Three situations: (a) and (b) high levels of glucose; (c) and (d) medium levels of glucose; (e) and (f) low levels of glucose. On the left of the vertical grey line, the calibration with uncertainty. On the right of the grey vertical line, the prediction for the best scenario. The horizontal red dashed lines correspond to the healthy levels of glucose $\mathrm{U}=160$ and $\mathrm{L}=90$. On the left column, the $k=100$ selected model outputs in the calibration procedure and the $k=100$ simulations over the next 4 hours for the maximum time in range scenario. On the right column, the same but only the mean and the $95 \%$ confidence intervals. . . . . . . . . . . . 220

10.4 The same as Figure 10.3 but for Patient 2. . . . . . . . . . . . 221

10.5 Probability to remain within the healthy levels of glucose $[70 \mathrm{mg} / \mathrm{dL}$, $180 \mathrm{mg} / \mathrm{dL}$ ] over the time, in the 6 studied cases. Green lines show the probability to be inside the healthy levels of glucose $[70,180]$; blue lines stand for the probability to reach levels of glucose greater than 180; red lines show the probability to reach lower levels of glucose than 70 . . . . . . . . . . . . . . . . . 224 



\section{List of Tables}

2.1 Theoretical values for the order of truncation $M$ using different values of the admissible error $\epsilon>$ in the context of Example 2.2. These theoretical values are compared with those, denoted by $\hat{M}$, obtained directly from our numerical computations. . . . . . . . . 4

2.2 Relative error for the mean $\mathrm{RE}($ Mean $)(t ; M)$ computed by $(2.55)$ for different values of $t$ and $M$ in the context of Example 2.3. . . .

2.3 Relative error for the standard deviation $\operatorname{RE}(\mathrm{SD})(t ; M)$ computed by (2.56) for different values of $t$ and $M$ in the context of Example

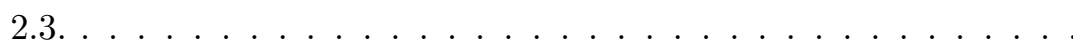

3.1 Some important families of RVs that satisfy inequality (3.5). . . . 52

3.2 Numerical values of the relative error (3.32) corresponding to the approximations of the mean of the solution SP to the random IVP (3.1) with $\alpha=0.3$ (Case I) at different values of $t$ and $M$ in the context of Example 3.5. . . . . . . . . . . . . . .

3.3 Numerical values of the relative error (3.33) corresponding to the standard deviation of the solution SP to the random IVP (3.1) with $\alpha=0.3$ (Case I) at different values of $t$ and $M$ in the context of Example 3.5. . . . . . . . . . . . . . . . .

3.4 Numerical values of the relative error (3.32) corresponding to the mean of the solution SP to the random IVP (3.1) with $\alpha=0.7$ (Case I) at different values of $t$ and $M$ in the context of Example 3.5. 66

3.5 Numerical values of the relative error (3.33) corresponding to the standard deviation of the solution SP to the random IVP (3.1) with $\alpha=0.7$ (Case I) at different values of $t$ and $M$ in the context of Example 3.5. . . . . . . . . . . . . . . . . 66 
3.6 Numerical values of the relative error (3.32) corresponding to the mean of the SP to the random IVP (3.1) with $\alpha=0.6$ (Case I) in different values of $t$ and $M$ in the context of Example 3.6. . . . . . 68

3.7 Numerical values of the relative error (3.33) corresponding to the standard deviation of the SP to the random IVP (3.1) with $\alpha=0.6$ (Case I) in different values of $t$ and $M$ in the context of Example 3.6. 68

4.1 Values of error $e_{M}(\hat{t})$ defined in (4.21) at the time instants $\hat{t}=$ $\{0.1,1,5\}$ in Example 4.1. The truncation order $M$ has been com-

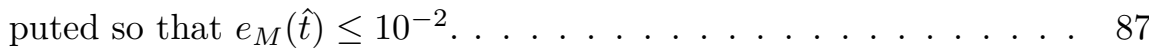

4.2 Values of errors defined in (4.22) to quantify the quality of approximations $\mu_{y^{M}}(t)$ and $\sigma_{y^{M}}^{2}(t)$, given by (4.23) and (4.24), respectively, to the exact mean and variance of the solution SP to random fractional IVP (4.3), respectively, for different values of the truncation order $M$ in the context of Example 4.1. The truncation order $M$ has been computed so that $e_{M}(\hat{t}) \leq 10^{-2}$. . . . . . . . . . . . 88

4.3 Values of error $e_{M}(\hat{t})$ defined in (4.21) at the time instants $\hat{t}=$ $\{0.05,0.2,1\}$ in Example 4.2. The truncation order $M$ has been computed so that $e_{M}(\hat{t}) \leq 10^{-2}$. . . . . . . . . . . . . 89

4.4 Values of errors defined in (4.22) to quantify the quality of approximations $\mu_{y^{M}}(t)$ and $\sigma_{y^{M}}^{2}(t)$, given by (4.23) and (4.24), respectively, to the exact mean and variance of the solution SP to random fractional IVP (4.3), respectively, for different values of the truncation order $M$ in the context of Example 4.2. The truncation order $M$ has been computed so that $e_{M}(\hat{t}) \leq 10^{-2}$. . . . . . . . . . . . 90

5.1 Cell counts $y_{i}$ of Rhodobacter Capsulatus anaerobic photosynthetic bacteria at the time instants $t_{i}$ (data retrieved from [110]). Values of the deterministic fitting $\left(y_{i}^{\text {det. }}\right)$ and random fractional fitting $y_{i}^{\text {random }}$. Goodness-of-fit measure for both approaches: MAPE (Mean Absolute Percentage Error). Example 5.2. . . . . . . . . . . . . . 105 
6.1 Values of the expectation of the solution SP of the random fractional IVP (6.1) computed by (6.15) with $M=10$ in the context of Example 6.1. ..................... 123

6.2 Values of the variance of the solution SP to the random fractional IVP (6.1) computed by (6.17) with $M=10$ in the context of Example 6.1. .................... 123

6.3 Relative error for the mean, given by (6.25), at different time instants for different orders of truncation $M$ in the context of Example 6.1. 125

6.4 Relative error for the variance, given by (6.26), at different time instants for different orders of truncation $M$ in the context of Example 6.1.

6.5 Values of $\lambda_{0}, \lambda_{1}, \lambda_{2}$ that solve the non-linear system of equations (6.24) (with $m_{1}$ and $m_{2}$ given in the corresponding columns) and determine the PDF $g(y)$ (and thus $f_{Y_{M}(t)}(y, t)$ ) given by $(6.22)$ for $M=30$ at different times instants $t=0,1,2, \ldots, 9$. This function approximates the $\operatorname{PDF} f_{Y(t)}(y, t)$ of the solution SP to random fractional IVP (6.1) for the order of the fractional derivative $\alpha=$ 1.9 in the context of Example 6.1. In the last column we give the probability that the solution lies in the interval $\left[y_{1}, y_{2}\right]$ being $\left.y_{1}=\mathbb{E}\left[Y_{M}(t)\right]-\sqrt{\mathbb{V}\left[Y_{M}(t)\right]}\right]$ and $\left.y_{2}=\mathbb{E}\left[Y_{M}(t)\right]+\sqrt{\mathbb{V}\left[Y_{M}(t)\right]}\right]$.

6.6 Relative error for the mean, given by (6.25), at different time instants for different orders of truncation $M$ in the context of Example 6.2. 129

6.7 Relative error for the variance, given by (6.26), at different time instants for different orders of truncation $M$ in the context of Example 6.2.

6.8 Relative error for the mean, given by (6.25), at different time instants for different orders of truncation $M$ in the context of Example 6.3. 133 
6.9 Relative error for the variance, given by (6.26), at different time instants for different orders of truncation $M$ in the context of Example 6.3. . . . . . . . . . . . . . . . . . 133

7.1 Approximations of the mean calculated by expressions (7.22) and (7.30) for different values of $n$ and different time instants $t$. Example

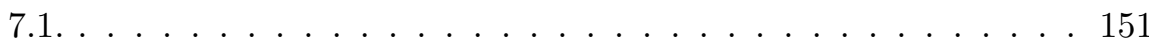

7.2 Approximations of the variance calculated by expressions (7.24) and (7.31) for different values of $n$ and different time instants $t$. Example 7.1. . . . . . . . . . . . . . . . . . . 152

7.3 Absolute errors for the approximations of the mean collected in Table 7.1. Example 7.1. . . . . . . . . . . . . . . . . . . . . . . . . 152

7.4 Absolute errors for the approximations of the variance collected in Table 7.2. Example 7.1. . . . . . . . . . . . . . . . . . . . . . 152

7.5 Values of parameters $\lambda_{0}, \lambda_{1}$ and $\lambda_{2}$ that, according to (6.22), determine the approximate 1-PDF using PME to the random fractional IVP (7.1) at different time instants $t=0.1,0.2,0.3,0.4$. Example 7.1.153

7.6 Values of the error (via the 2-norm) between the exact 1-PDF, given in (7.33), and its approximations obtained via PME at different times $t=0.1,0.2,0.3,0.4$. Example 7.1. . . . . . . . . . . . 154

7.7 Approximations for the mean calculated by expressions (7.22) and (7.35) for different values of $n$ at different time instants. Example 7.2.157

7.8 Approximations for the variance calculated by expressions (7.24) and (7.36) for different values of $n$ at different time instants. Example 7.2.158

7.9 Absolute errors for the mean. Example 7.2. . . . . . . . . . . . 158

7.10 Absolute error for the variance. Example 7.2. . . . . . . . . . . 158

7.11 Values of the parameters $\lambda_{0}, \lambda_{1}$ and $\lambda_{2}$ to construct the approximate 1-PDF using PME for the IVP (7.34) at different $t=0.2,0.4,0.6,0.8 .158$ 
8.1 Sample size, number and percentage of susceptible carriers of any meningococcus in Spain in Dec 2011 and Dec 2012. . . . . . . . . . 166

8.2 Percentage of carriers of the genotype Men W-135 in Spain in Dec 2011 and Dec 2012 among the carriers. . . . . . . . . . . . 166

8.3 95\% CI of the data surveys using the probability mass function of Beta-Binomial distributions given by $(8.14)-(8.17)$. . . . . . . . 174

8.4 95\% CI of the data surveys using the probability mass function of Beta-Binomial distributions given by $(8.20)-(8.21)$. . . . . . . . 175

8.5 Model fitting to $N$ samples of the data's probability distributions. 176

8.6 Probability that the percentage of carriers of Men W-135 be greater than $5 \%$. . . . . . . . . . . . . . . . 180

9.1 Volume of breast tumor cells using xenograft technique at different days, [123] $\left(\tilde{m}_{1, \tilde{n}}\right)$ together with the assigned variance $\left(\tilde{\sigma}_{\tilde{n}}^{2}\right)$ and second moment $\left(\tilde{m}_{2, \tilde{n}}\right)$. . . . . . . . . . . . . . . . . . 191

9.2 Values of $\lambda_{0}^{\tilde{n}}, \lambda_{1}^{\tilde{n}}$ and $\lambda_{2}^{\tilde{n}}$ obtained solving the system of nonlinear equations given in (9.8) the different values of $\tilde{n}$. . . . . . . . . . 192

9.3 Values of parameters $\lambda_{1}^{A}, \lambda_{2}^{A}, a_{2}, \lambda_{1}^{B}, \lambda_{2}^{B}, b_{2}$ that minimize the fitness function FF using Particle Swarm Optimization algorithm. . . . . 199

10.1 Units of the model variables, data of the patient and parameters. . 210

10.2 Minute when the insulin should be administered, which quantity and, after 15 minutes, the amount of carbohydrates the patient should eat for his/her best recommendation. . . . . . . . . . . . . . 219

10.3 Patient 1. Mean and CI95\% of the model parameter values in the situations HL (high level of glucose), LL (low level of glucose), IL (levels of glucose inside the healthy range). . . . . . . . . . . . 222 
10.4 Patient 2. Mean and CI95\% of the model parameter values in the situations HL (high level of glucose), LL (low level of glucose), IL (levels of glucose inside the healthy range). . . . . . . . . . 222 


\section{Chapter}

\section{General Introduction}

Uncertainty quantification is an emerging area in mathematics whose main goal is determining information about the uncertainty in the outputs of a mathematical system (usually formulated via dynamical systems), from the available information about the randomness in the inputs. In the setting of mathematical modelling, uncertainty quantification seeks for better explaining the model answer taking into account the variability often met in natural and physical phenomena, $[47,66$, $23,44]$.

This $\mathrm{PhD}$ thesis focusses on developing new uncertainty quantification techniques in two mathematical areas, fractional calculus and mathematical modelling. On the one hand, in Chapters 2, 3, 4, 5, 6 and 7, some foundations of random fractional calculus are extended using the mean square (m.s.) calculus for random variables and stochastic processes. On the other and, in Chapters 8, 9, 10, new computational techniques to deal with uncertainty quantification are developed in the setting of three models describing specific biological dynamics.

The ubiquity of differential equations to model successfully real problems in different areas as Biology, Physics, Economics, Epidemiology, etc., is well-known. When 
they are applied to describe the dynamics of physical phenomena on the basis of sampled data, the parameters in the model formulation (coefficients, forcing term, initial/boundary conditions) need to be established. This is usually done by assigning a nominal or averaged value (deterministic) to each model parameter.

Nowadays, it is widely accepted that the behaviour of many physical phenomena is governed by chance. Thus, it is not appropriate to describe them assigning deterministic values for the model parameters, but also considering the randomness into their physical formulations. In this regard, it is well-known that the trajectory of a rocket is determined by the randomness of the initial speed; the electric power exhibits visible changes that behave irregularly; the value of assets in financial markets is often very volatile; these are just few examples where it is reasonable to consider uncertainty. From this point of view, it is natural to take advantage of the powerful effectiveness of deterministic differential equations for describing physical phenomena and interpreting the parameters of the model as random variables (RVs) or stochastic processes (SPs) rather than constants and functions, respectively.

Two classes of differential equations where uncertainties are involved in their formulation are often distinguished, namely, Stochastic Differential Equations (SDEs) and Random Differential Equations (RDEs). In dealing with SDEs, the uncertainty is forced by a SP whose sample behaviour is quite irregular, such as the Wiener process, whose trajectories are nowhere differentiable. In this case, the underlying probabilistic pattern is Gaussian. The analytic and numerical treatment of SDEs requires the so-called Itô-Doob Calculus [101, 97, 83]. Some interesting applications based on this approach are reported in $[49,92,126]$, for instance.

RDEs appear as natural generalizations of their deterministic counterpart, namely deterministic differential equations, since they are just formulated by randomizing their parameters. This is advantageous on both theoretical and practical levels. Some recent contributions about RDEs are [53, 122, 69]. It is important to point out that there are different approaches to deal with RDEs, but in these pages we will follow the so-called mean square (m.s.) approach [109]. This approach is based upon a strong stochastic type-convergence, termed m.s. convergence, whose main advantage is that the results established in m.s. sense are also valid in other 
important types of stochastic convergences, namely, convergence in probability and convergence in distribution. From a theoretical point of view, solving RDEs following the m.s. approach, takes advantage of powerful classical Newton-Leibniz Calculus. Indeed, in this context the probabilistic concepts of m.s. continuity, differentiability, integrability of a SP can be characterized in terms of classical continuity, differentiability, integrability to its associated correlation function, which is a two-dimensional deterministic function [109, 119, 95]. Additionally, the m.s. convergence possesses a distinctive property, which will be used along this $\mathrm{PhD}$ thesis (see Proposition 2.10), that makes it especially suitable to construct reliable approximations of the mean and variance of the solution SP of RDEs. Some recent papers where RDEs are studied using the m.s. calculus are [59, 96, 81], for instance.

Apart from considering uncertainty in differential equations, mathematical modelling may be improved when non-integer order derivatives are also introduced. This kind of derivatives are commonly named as fractional derivatives. Over the last few decades, deterministic fractional differential equations are having an important impact on both the theory and applications of mathematics. Despite the physical meaning of the fractional derivative is still under discussion, fractional differential equations are gaining influence in mathematical modelling because their success in describing phenomena having a microscopic complex behaviour whose macroscopic dynamics can not be properly modelled using the classical deterministic derivative $[82,58]$.

Some areas where deterministic fractional differential equations have demonstrated to be useful tools include Viscoelasticity Materials, Fluid Flows, Solute Transport, etc., [82]. Many authors attribute the success of fractional differential equations to the fact that many of the physical processes related to complex systems possess non-local dynamics involving long-memory in time, and the fractional integral and fractional derivative operators do have some of these characteristics [82, 58]. So it is reasonable to introduce fractional derivatives in the random model formulation. Moreover, fractional derivatives allow more flexibility when fitting the solution of a random fractional differential equation (RFDE) to sample data $[82,79,77]$. 
This leads to the emergent and attractive combination of fractional calculus and SDEs or RDEs, where two powerful tools, namely Fractional Calculus and Itô Stochastic/m.s. Random Calculus, are merged. Some recent contributions dealing with interesting problems related to fractional SDEs and fractional RDEs include $[49,92,65,9]$ and $[89,88]$, respectively. In [89, 88] interesting existence and uniqueness results, based on the so-called sample path and $\mathrm{L}^{p}$-approaches, for initial value problems formulated through fractional RDEs have been presented. While a number of contributions have dealt with fractional SDEs, see for example $[65,9]$, to the best of our knowledge there is a gap in the study regarding fractional RDEs. Along this $\mathrm{PhD}$ thesis we will focus on Random Fractional Differential Equations (RFDEs) in m.s. sense only. Chapters 2, 5, 4, 3, 6 and 7 are mainly based on solving some types of RFDEs.

From the modelling point of view, the combination of computational techniques to quantify the uncertainty with mathematical models formulated by fractional differential or difference equations, allow us to have a reliable description of the real problem under study, since fractional derivatives can describe physical processes related to complex systems involving memory effects and non-local dynamics that classical derivatives cannot. To show this applicability, in this dissertation we also focus on study new computational techniques to quantify uncertainty in mathematical models formulated via fractional derivatives (Chapter 8).

To develop new computational strategies to quantify the uncertainty, it is necessary to test them in mathematical models. However, computing the fractional derivatives included in these mathematical formulations requires computational resources and sometimes this fact limits the proper evaluation of the algorithm. Thus, it is always recommended to test previously the new techniques in models where classical derivatives (non-fractional) are involved. That is the reason why in the two last Chapters in this $\mathrm{PhD}$ thesis we focus on developing techniques to quantify the uncertainty in models defined by non-fractional derivatives (Chapters 9 and 10). In future works, we aim at extending these techniques to fractional models with randomness.

\section{Thesis outline}


This PhD thesis is structured in ten chapters. To follow the connecting thread along this dissertation, we write a motivation at the beginning of each chapter.

Firstly, Chapter 2 is devoted to extend the main deterministic fractional operators in the random framework, concretely the Riemann-Liouville integral and the Caputo derivative. Moreover, a random linear fractional differential equation, where the order of the derivative, $\alpha$, lies in $[0,1[$, is solved. The solution is expressed by a random generalized power series. Conditions on the random input parameters to guarantee the convergence in the m.s. sense of the power series solution are established. Also, closed expressions for the mean and for the variance for the solution SP are obtained. In Chapter 3, we study the same random linear differential equation, but in this case the order of the derivative $\alpha$ lies in $[0,2[$. More general conditions to guarantee the convergence in m.s. sense are given. Addressing the same random linear fractional differential equation, in Chapter 4 the first probability density function of the solution SP where $\alpha$ lies in $[0,1[$ is computed and conditions to guarantee the convergence are established. The computation of the first probability density function is advantageous. From this function we can obtain a complete description of the solution SP, since for it one can straightforwardly compute one-dimensional moments, in particular, the mean and the variance, as well as to determine the probability that the solution lies in an interval of specific interest.

Chapters 5 and 6 are devoted to study a random non-autonomous linear fractional differential equation where the order of the derivative, $\alpha$, lies in $[0,1[$ and $[0,+\infty[$, respectively. Solutions given by random generalized power series are obtained and mild conditions to guarantee the convergence in m.s. sense are established. Moreover expressions for the mean and for the variance of the solution SP are computed. An approximation of the first probability density function of the solution $\mathrm{SP}$ is also addressed in Chapter 6 using Principle of Maximum Entropy.

In all the mentioned chapters, so far, the exact solution for random fractional differential equations have been obtained, however it is not always possible. Thus, like in the deterministic setting, numerical methods to compute approximate solutions are required. Chapter 7 is devoted to extend the classical fractional Euler forward-like method into the random framework, concretely in m.s. sense. 
To illustrate the applicability of random fractional calculus in mathematical modelling, Chapter 8 is devoted to study probabilistically the outbreaks of meningococcus W-135 infections in Spain combining a discrete Susceptible-Carrier-Susceptible epidemiological model and a fractional Lotka-Volterra competition model. A confidence interval is constructed to predict the carriers of meningococcus $\mathrm{W}-135$ among the Spanish population and the probability to achieve a $5 \%$ of the carriers is also computed, since, according with the physicians, exceeding this percentage may be concerning.

Nevertheless, obtaining a confidence interval is not always enough if we want to have a complete probabilistic description of the phenomena under study. In Chapter 9 , we focus on developing a computational approach to obtain the first probability density function for a randomized non-fractional logistic model to describe the breast tumour growth by combining the so called Random Variable Transformation method and the Principle of Maximum Entropy. In future contributions we plan to extend this technique to the fractional framework.

Finally, Chapter 10, focusses on describing a computational approach to quantify the uncertainty in real data and in the corresponding model that aims at describing it. Specifically, in this chapter we will apply this technique to diabetes. A recommendation of insulin shots and carbohydrates intakes to a patient is given in order to maintain her/his glucose level in a healthy range. 


\section{Chapter}

\section{Extending the deterministic}

Riemann-Liouville and Caputo operators to the random framework: a mean square approach with applications to solve a random linear fractional differential equation

This chapter extends both the deterministic fractional Riemann-Liouville integral and the Caputo fractional derivative to the random framework using the mean square random calculus. Characterizations and sufficient conditions to guarantee

the existence of both fractional random operators are given. Assuming mild conditions on the random input parameters, the solution of the general random fractional linear differential equation, whose fractional order of the derivative is $\alpha \in] 0,1]$, is constructed. The approach is based on a mean square chain rule together with the random Frobenius method. Closed form expressions to construct reliable approximations for the mean and for the variance of the solution stochastic process are also given. Several examples illustrating the theoretical results are included. 


\subsection{Introduction}

As indicated in Chapter 1, the extension of fractional calculus into the random framework generates a suitable tool in mathematical modelling. On the one hand, the concept of fractional derivative improves the modelling of some physical phenomena, as material viscoelasticity or memory effects. On the other hand, the uncertainty presented in some measurements is required in the modelling process to get exhaustive results. In the first chapters of this $\mathrm{PhD}$ thesis we will focus on generalizing some concepts of fractional calculus into the random framework, specifically in the m.s. sense.

The goal of this chapter is twofold. Firstly, to extend some important concepts and results that belong to the deterministic fractional calculus to the random framework using the so-called m.s. approach. Secondly, to show some applications of the m.s. random fractional calculus to solve fractional differential equations with uncertainties.

This chapter is organized as follows. Section 2.2 contains the main results related to the so-called $\mathrm{L}^{2}$-random calculus, also termed m.s. calculus that will be required throughout this chapter. In Section 2.3, we extend the concept of the fractional Riemann-Liouville integral and fractional Caputo derivative to the m.s. random calculus. Characterizations of these two important random fractional operators, in terms of the correlation function of the involved second-order stochastic process, are explicitly given. Section 2.4 is addressed to show how the random Frobenius power series method can be applied to successfully solve the complete random linear fractional differential equation under very general hypotheses and assuming randomness in all its input parameters (initial condition, forcing term and diffusion coefficient). General explicit formulas for computing accurate approximations of the mean, variance and covariance functions of the solution stochastic process to the complete random linear fractional differential equation are given in Section 2.5. Section 2.6 is devoted to exhibit several illustrative examples. Conclusions are drawn in Section 2.7. 


\subsection{Preliminaries about mean square random calculus}

For the sake of completeness, henceforth we will summarize the main definitions and results that will be used throughout this chapter. A comprehensive survey of them can be found in [7, Chapter 1], [109, Chapter 4] and [119]. A complex random variable $(\mathrm{RV}), X: \Omega \rightarrow \mathbb{C}$, defined on a complete probability space $(\Omega, \mathfrak{F}, \mathbb{P})$ is said to be of order $p \geq 1$ ( $p$-RV, for short), if $\mathbb{E}\left[|X|^{p}\right]<+\infty$, where $\mathbb{E}[\cdot]$ denotes the expectation operator. The space $\mathrm{L}^{p}(\Omega)$ of all $p$-RVs endowed with the norm

$$
\|X\|_{p}=\left(\mathbb{E}\left[|X|^{p}\right]\right)^{1 / p},
$$

is a Banach space [7, page 9]. The convergence in $\mathrm{L}^{p}(\Omega)$, usually called convergence in $p$-th mean, it is naturally inferred by the $p$-norm (2.1), i.e., a sequence of RVs $\left\{X_{n}: n \geq 0\right\}$ in $\mathrm{L}^{p}(\Omega)$ is said to be $p$-th mean convergent to $X \in \mathrm{L}^{p}(\Omega)$ if, and only if, $\left\|X_{n}-X\right\|_{p} \underset{n \rightarrow+\infty}{\longrightarrow} 0$.

Given $\emptyset \neq \mathcal{U} \subset \mathbb{R}$, a family of RVs indexed by $u \in \mathcal{U}, X(u) \equiv\{X(u): u \in \mathcal{U}\}$ is called a stochastic process (SP). Throughout this document, we will take $\mathcal{U}=$ $\left[0,+\infty\left[\right.\right.$. If $\mathbb{E}\left[|X(u)|^{p}\right]<+\infty$ for each $u \in \mathcal{U}$, then $X(u)$ is said to be a $p$-stochastic process ( $p$-SP, for short). The definitions of $p$-continuity, $p$-differentiability and $p$-integrability of $p$-SPs in $\mathrm{L}^{p}(\Omega)$-spaces are the usual ones derived from the $p$-norm (2.1) in Banach spaces.

A significant case corresponds to $p=2$. In fact, it can be seen that $\left(\mathrm{L}^{2}(\Omega),\|\cdot\|_{2}\right)$ is a Hilbert space defined by

$$
\mathrm{L}^{2}(\Omega)=\left\{X: \Omega \longrightarrow \mathbb{R}:\left(\mathbb{E}\left[X^{2}\right]\right)^{1 / 2}<+\infty\right\}, \quad\|X\|_{2}=\left(\mathbb{E}\left[X^{2}\right]\right)^{1 / 2},
$$

with the inner product $\langle X, Y\rangle=\mathbb{E}[X Y], X, Y \in \mathrm{L}^{2}(\Omega)$.

The convergence associated to 2-norm is usually referred to as m.s. convergence. Hereinafter, $X_{n} \underset{n \rightarrow+\infty}{\stackrel{\text { m.s. }}{\longrightarrow}} X$ will denote a sequence, $\left\{X_{n}: n \geq 0\right\}$, of RVs in $\mathrm{L}^{2}(\Omega)$ such that is m.s. convergent to $X$ as $n \rightarrow+\infty$. 
Chapter 2. Riemann-Liouville and Caputo operators in mean square sense: solving a random linear fractional differential equation

As it shall see later, the Schwarz's inequality

$$
\mathbb{E}[|X Y|] \leq\|X\|_{2}\|Y\|_{2}, \quad X, Y \in \mathrm{L}^{2}(\Omega),
$$

will be used extensively throughout the chapter. Another important inequality that will be subsequently applied is the Jensen's inequality. If $f: \mathbb{R} \longrightarrow \mathbb{R}$ is a convex mapping and $X$ is a RV, then

$$
f(\mathbb{E}[X]) \leq \mathbb{E}[f(X)]
$$

provided all the above involved moments exist.

Although m.s. convergence is an important type of stochastic convergence, some basic operational rules do not fulfil unless additional hypotheses are assumed. Now, we prove a result in this respect that will be required later.

Proposition 2.1 Let $X$ be a bounded $R V$ in $\mathrm{L}^{2}(\Omega)$, i.e., there exist constants $x_{1}$ and $x_{2}$ such that $x_{1} \leq X(\omega) \leq x_{2}$, for all $\omega \in \Omega$, and let us assume that $Z_{n}$ converges in the m.s. sense to $Z$. Then, $X Z_{n} \underset{n \rightarrow \infty}{\stackrel{m . s .}{\longrightarrow}} X Z$.

Proof. Let $\hat{x}=\max \left\{\left|x_{1}\right|,\left|x_{2}\right|\right\}<+\infty$, and observe that

$$
\begin{aligned}
0 & \leq\left(\left\|X Z_{n}-X Z\right\|_{2}\right)^{2}=\mathbb{E}\left[|X|^{2}\left|Z_{n}-Z\right|^{2}\right] \leq|\hat{x}|^{2} \mathbb{E}\left[\left|Z_{n}-Z\right|^{2}\right] \\
& =|\hat{x}|^{2}\left(\left\|Z_{n}-Z\right\|_{2}\right)^{2} \stackrel{[n \rightarrow+\infty]}{\longrightarrow} 0,
\end{aligned}
$$

since $\left\{Z_{n}\right\}$ converges in the m.s. sense to $Z$ as $n \rightarrow+\infty$. Then, the result is proved.

2-RVs are met in the most of physical problems including randomness where usually the variability (variance) is finite. Moreover, the particular concept of 2-stochastic process (2-SP) is also required when RDE are involved. Recall that given $\mathcal{U} \subset \mathbb{R}$, then $X(t)$ is called a 2 -SP if $X(t) \equiv\{X(t): t \in \mathcal{U}\}$ is a 2 -RV for every $t \in \mathcal{T}$.

As we shall see below, the concept of m.s. differentiability of a 2-SP will be required for introducing the random m.s. fractional Caputo derivative. We recall that a 2-SP $X(t)$ is m.s. differentiable at $t_{0} \in \mathcal{U}$, with m.s. derivative $X^{\prime}\left(t_{0}\right)$, if 


$$
\lim _{h \rightarrow 0}\left\|\frac{X\left(t_{0}+h\right)-X\left(t_{0}\right)}{h}-X^{\prime}\left(t_{0}\right)\right\|_{2}=0 \quad t_{0}, t_{0}+h \in \mathcal{U} .
$$

If $X^{\prime}(t)$ exists for $t \in \mathcal{U}$, it is also a 2-SP. Higher order m.s. derivatives, denoted by $\frac{\mathrm{d}^{n} X(u)}{\mathrm{d} u^{n}} \equiv X^{(n)}(u), n \geq 1$, are defined analogously. The next result provides information of the m.s. derivative of the product of a deterministic function with a SP.

Theorem 2.1 [109] If $f(t)$ is a deterministic differentiable function at $t_{0}$ and the 2-SP $X(t)$ is m.s. differentiable at $t_{0}$, then the 2 -SP $X(t)=f(t) X(t)$ is m.s. differentiable at $t_{0}$ and its m.s. derivative is given by $U^{\prime}(t)=f\left(t_{0}\right) X^{\prime}\left(t_{0}\right)+$ $f^{\prime}\left(t_{0}\right) X\left(t_{0}\right)$.

When two o more RVs are involved, statistical dependence is an important matter. The following property will be used through this chapter.

Proposition 2.2 [63, page 92] Let $f, g: \mathbb{R} \longrightarrow \mathbb{R}$ be measurable mappings and $X, Y: \Omega \longrightarrow \mathbb{R}$ independent $R V s$. Then, $f(X)$ and $g(Y)$ are independent $R V$ s and $\mathbb{E}[f(X) g(Y)]=\mathbb{E}[f(X)] \mathbb{E}[g(Y)]$, provided the above expectations exist.

Furthermore, to deal with statistical dependence it is convenient to introduce the definition of correlation function. If $X(u)$ is a 2-SP, then for each $u_{1}, u_{2}>0$, the two-dimensional deterministic function $\Gamma_{X}\left(u_{1}, u_{2}\right)=\mathbb{E}\left[X\left(u_{1}\right) X\left(u_{2}\right)\right]$ is called the correlation function associated to $X(u)$. The correlation function $\Gamma_{X}\left(u_{1}, u_{2}\right)$ of a 2-SP $X(u)$ always exists since

$$
\left|\Gamma_{X}\left(u_{1}, u_{2}\right)\right|=\left|\mathbb{E}\left[X\left(u_{1}\right) X\left(u_{2}\right)\right]\right| \leq \mathbb{E}\left[\left|X\left(u_{1}\right) X\left(u_{2}\right)\right|\right] \leq\left\|X\left(u_{1}\right)\right\|_{2}\left\|X\left(u_{2}\right)\right\|_{2}<+\infty .
$$

Notice that in the latter expression, we have applied first the Jensen inequality (2.4) with $f(x)=|x|$, which is a convex function, and secondly, the Schwarz's inequality (2.3). Finally, since $X\left(u_{1}\right), X\left(u_{2}\right) \in \mathrm{L}^{2}(\Omega)$, hence the norms $\left\|X\left(u_{1}\right)\right\|_{2}$ and $\left\|X\left(u_{2}\right)\right\|_{2}$ are finite. 
Chapter 2. Riemann-Liouville and Caputo operators in mean square sense: solving a random linear fractional differential equation

We point out that many m.s. properties, such as $\|\cdot\|_{2}$-continuity, $\|\cdot\|_{2}$-differentiability and $\|\cdot\|_{2}$-integrability of a 2-SP, say $X(u)$, can be directly characterized through its correlation function $\Gamma_{X}\left(u_{1}, u_{2}\right)$ [109, Chapter 4].

Apart from the correlation function, other important functions that will be used in the subsequent sections to study the statistical dependence of the involved RVs are the covariance function, $\mathbb{C}_{X}\left(u_{1}, u_{2}\right)$ of 2-SP $X(u)$, and the cross-covariance function, $\mathbb{C}_{X, Y}\left(u_{1}, u_{2}\right)$, of two second-order SPs $X(u)$ and $Y(u)$. These functions are defined by

$$
\begin{aligned}
\mathbb{C}_{X}\left(u_{1}, u_{2}\right) & =\Gamma_{X}\left(u_{1}, u_{2}\right)-\mathbb{E}\left[X\left(u_{1}\right)\right] \mathbb{E}\left[X\left(u_{2}\right)\right], \\
\mathbb{C}_{X, Y}\left(u_{1}, u_{2}\right) & =\mathbb{E}\left[X\left(u_{1}\right) Y\left(u_{2}\right)\right]-\mathbb{E}\left[X\left(u_{1}\right)\right] \mathbb{E}\left[Y\left(u_{2}\right)\right],
\end{aligned}
$$

respectively.

We have seen that the m.s. derivative of a 2-SP, say $X(u)$, and its higher order ones, if they exist are also 2-SPs. It can be shown that their correlation functions are determined simply in terms of the correlation function of $X(u)$ [109, page 97]. Specifically, if $X(u)$ is $n$-times m.s. differentiable, then

$$
\Gamma_{X^{(n)}}\left(u_{1}, u_{2}\right)=\frac{\partial^{2 n} \Gamma_{X}\left(u_{1}, u_{2}\right)}{\partial u_{1}^{n} \partial u_{2}^{n}} .
$$

The following result gives a characterization of the existence of the m.s. Riemann integral of a 2-SP, in terms of the existence of a two-dimensional integral involving the correlation function of the 2-SP.

Proposition 2.3 ([109, Theorem 4.5.1]) Let $g(u, w)$ be a deterministic Riemann integrable function on the real interval $u \in[c, d]$, for every $w \in \mathcal{W} \subset \mathbb{R}$, and let $X(u)$ be a 2-SP. Then, the 2-SP defined by

$$
Y(w)=\int_{c}^{d} g(u, w) X(u) \mathrm{d} u, \quad w \in \mathcal{W},
$$


exists if and only if, the deterministic double Riemann integral

$$
\int_{c}^{d} \int_{c}^{d} g\left(u_{1}, w\right) g\left(u_{2}, w\right) \Gamma_{X}\left(u_{1}, u_{2}\right) \mathrm{d} u_{1} \mathrm{~d} u_{2}
$$

exists and is finite.

The following consequence of the previous proposition will be used later.

Remark 2.1 In the particular case that $w=d \in \mathcal{W} \subset \mathbb{R}$ in Proposition 2.3, the $R V$

$$
Y \equiv Y(d)=\int_{c}^{d} g(u, d) X(u) \mathrm{d} u
$$

is well-defined as a 2-RV, if and only if, the deterministic double Riemann integral

$$
\int_{c}^{d} \int_{c}^{d} g\left(u_{1}, d\right) g\left(u_{2}, d\right) \Gamma_{X}\left(u_{1}, u_{2}\right) \mathrm{d} u_{1} \mathrm{~d} u_{2}
$$

exists and is finite.

A key result, that will be used in this chapter to construct a random generalized power series solution to the random fractional linear differential equation with a random initial condition, is the following chain rule [46]. This rule allows us to compute the m.s. derivative of a 2-SP resulting from the composition of a differentiable deterministic function and a m.s. differentiable 2-SP.

Theorem 2.2 Let $g$ be a deterministic continuous function on $\left[a_{1}, a_{2}\right]$ such that $g^{\prime}(t)$ exists and is finite at some point $t \in\left[a_{1}, a_{2}\right]$. If $\{X(v): v \in \mathcal{V}\}$ is a 2-SP such that

i) The interval $\mathcal{V}$ contains the range of $g, g\left(\left[a_{1}, a_{2}\right]\right) \subset \mathcal{V}$.

ii) $X(v)$ is m.s. differentiable at the point $g(t)$.

iii) The m.s. derivative of $X(v), \frac{\mathrm{d} X(v)}{\mathrm{d} v}$, is m.s. continuous on $\mathcal{V}$. 
Chapter 2. Riemann-Liouville and Caputo operators in mean square sense: solving a random linear fractional differential equation

Then, the 2-SP, $X(g(t))$, is m.s. differentiable at $t$ and the m.s. derivative is given by

$$
\frac{\mathrm{d} X(g(t))}{\mathrm{d} t}=\left.\frac{\mathrm{d} X(v)}{\mathrm{d} v}\right|_{v=g(t)} g^{\prime}(t) .
$$

Also connected with the previous result and, as it shall be seen later, we will require to apply the m.s. derivative of a random power series in order to formally construct the solution of the random fractional linear differential equation with a random initial condition. For this purpose we will use the following result:

Proposition 2.4 [45, page 1260] Let $\mathcal{V} \subset \mathbb{R}$ be an interval, $m \geq m_{0} \geq 0$ a non-negative integer and $\left\{U_{m}(v): v \in \mathcal{V}, m \geq m_{0}\right\}$ be a sequence of 2-SPs such that

i) $U_{m}(v)$ is m.s. differentiable on $\mathcal{V}$.

ii) The m.s. derivative, $U_{m}^{\prime}(v)$, is m.s. continuous on $\mathcal{V}$.

iii) $U(v)=\sum_{m \geq m_{0}} U_{m}(v)$ is m.s. convergent on $\mathcal{V}$.

iv) $\sum_{m \geq m_{0}} U_{m}^{\prime}(v)$ is m.s. uniformly convergent on $\mathcal{V}$.

Then, the 2-SP, $U(v)$, is m.s. differentiable at every $v \in \mathcal{V}$ and

$$
U^{\prime}(v)=\sum_{m \geq m_{0}} U_{m}^{\prime}(v)
$$

Apart of the key results described above with the differentiability in m.s. sense of 2-SP, special deterministic functions as Euler gamma and beta functions will be required in the following chapters. They are defined as

$$
\begin{gathered}
\Gamma(\alpha):=\int_{0^{+}}^{\infty} \mathrm{e}^{-v} v^{\alpha-1} \mathrm{~d} v, \quad \alpha>0, \\
\mathrm{~B}\left(\alpha_{1}, \alpha_{2}\right):=\int_{0}^{1} v^{\alpha_{1}-1}(1-v)^{\alpha_{2}-1} \mathrm{~d} v, \quad \alpha_{1}, \alpha_{2}>0,
\end{gathered}
$$


respectively. They are related by the following well-known relationship

$$
\mathrm{B}\left(\alpha_{1}, \alpha_{2}\right)=\frac{\Gamma\left(\alpha_{1}\right) \Gamma\left(\alpha_{2}\right)}{\Gamma\left(\alpha_{1}+\alpha_{2}\right)}, \quad \alpha_{1}, \alpha_{2}>0 .
$$

The so-called duplication formula of the deterministic gamma function

$$
\Gamma(\alpha+1)=\alpha \Gamma(\alpha), \quad \alpha>0,
$$

will also be required later. Although these functions and relationships can be extended for $\alpha, \alpha_{1}$ and $\alpha_{2}$ lying in the whole complex plane except the negative integers, here they will only be applied when $\alpha>0, \alpha_{1}>0$ and $\alpha_{2}>0$. Also, the following asymptotic approximation to the gamma function will be need later [60, page 227]

$$
\Gamma(x+1) \approx x^{x} \mathrm{e}^{-x} \sqrt{2 \pi x}, \quad x \rightarrow+\infty .
$$

Notice that this approximation is just a generalization of the celebrated Stirling's formula.

We conclude this section by stating a technical result related to the convergence of double series that will be applied to develop the numerical examples exhibited in Section 2.6.

Proposition 2.5 [93, Lemma 9.1, Chapter 9] A double series $\sum_{m \geq m_{0}} \sum_{n \geq n_{0}} a_{m n}$ is absolutely convergent if and only if the following conditions hold

(i) There are $\left(m_{0}, n_{0}\right) \in \mathbb{N} \times \mathbb{N}$ and $\alpha_{0}>0$ such that $\sum_{m=m_{0}}^{M} \sum_{n=n_{0}}^{N}\left|a_{m n}\right| \leq$ $\alpha_{0}$ for all $M \geq m_{0}, N \geq n_{0}$.

(ii) Each row-series and each column-series are absolutely convergent.

\subsection{Mean square random fractional differential and integral opera- tors}

This section is addressed to introduce the random Riemann-Liouville fractional integral and the random Caputo fractional derivative in the m.s. sense. As it shall 
Chapter 2. Riemann-Liouville and Caputo operators in mean square sense: solving a random linear fractional differential equation

see later, both random fractional operators extend their deterministic counterparts. Their definitions are based on the random m.s. calculus. Firstly, we give the definition of the m.s. random Riemann-Liouville fractional integral.

Definition 2.1 Let $\mathcal{D}=[a, b],-\infty<a<b<+\infty$, be a finite interval of the real line, $\mathbb{R}$. Let $\{X(t): t \in \mathcal{D}\}$ be a 2 -SP. The random mean square (left-sided) Riemann-Liouville fractional integral of $X(t), \mathrm{J}_{a+}^{\alpha} X$, of order $\alpha>0$ is defined by

$$
\left(\mathrm{J}_{a+}^{\alpha} X\right)(t):=\frac{1}{\Gamma(\alpha)} \int_{a}^{t}(t-u)^{\alpha-1} X(u) \mathrm{d} u, \quad t \in \mathcal{D}=[a, b],
$$

where $\Gamma(\alpha)$ denotes the deterministic gamma function given in (2.8).

Notice that the integral that appears in the right-hand side of (2.13) is understood in the m.s. Riemann integral sense introduced in Section 2.2.

Remark 2.2 Analogously to Definition 2.1, we can define the random m.s. (rightsided) Riemann-Liouville fractional integral of a 2-SP, $X(t)$, as

$$
\left(\mathrm{J}_{b-}^{\alpha} X\right)(t):=\frac{1}{\Gamma(\alpha)} \int_{t}^{b}(u-t)^{\alpha-1} X(u) \mathrm{d} u .
$$

Throughout this chapter, the random m.s. (left-sided) Riemann-Liouville fractional integral will be used only.

Keeping the notation of Definition 2.1, and applying Remark 2.1 with the following identification $d=t \in \mathcal{D}$ an arbitrary but fixed number and $g(u, d)=$ $(d-u)^{\alpha-1} / \Gamma(\alpha)$, one deduces the following characterization of the existence of the random m.s. (left-sided) Riemann-Liouville fractional integral of a 2-SP $\{X(t): t \in \mathcal{D}\}$.

Proposition 2.6 Let $\{X(t): t \in \mathcal{D}\}$ be a 2 -SP with correlation function $\Gamma_{X}(\cdot, \cdot)$. Then, its random m.s. (left-sided) Riemann-Liouville fractional integral, denoted by $\left(\mathrm{J}_{a+}^{\alpha} X\right)(t), \alpha>0$, exists in the m.s. sense if, and only if the following deterministic 
double Riemann integral

$$
\int_{a}^{t} \int_{a}^{t}\left(t-u_{1}\right)^{\alpha-1}\left(t-u_{2}\right)^{\alpha-1} \Gamma_{X}\left(u_{1}, u_{2}\right) \mathrm{d} u_{1} \mathrm{~d} u_{2}
$$

exists and is finite for each $t \in \mathcal{D}$.

Now we give a sufficient condition in order to guarantee the existence of random m.s. (left-sided) Riemann-Liouville fractional integral, $\left(\mathrm{J}_{a+}^{\alpha} X\right)(t)$.

Proposition 2.7 Let $\alpha>0$ and $\{X(t): t \in \mathcal{D}\}$ be a 2-SP such as

$$
\int_{a}^{t}(t-u)^{\alpha-1}\|X(u)\|_{2} \mathrm{~d} u<+\infty
$$

Then, the random m.s. (left-sided) Riemann-Liouville fractional integral $\left(\mathrm{J}_{a+}^{\alpha} X\right)(t)$ exists.

Proof. By Proposition 2.6, it is enough showing that the double deterministic integral (2.14) is absolutely convergent. This follows by applying inequality (2.5) and Fubini's theorem

$$
\begin{aligned}
& \int_{a}^{t} \int_{a}^{t}\left|\left(t-u_{1}\right)^{\alpha-1}\left(t-u_{2}\right)^{\alpha-1} \Gamma_{X}\left(u_{1}, u_{2}\right)\right| \mathrm{d} u_{1} \mathrm{~d} u_{2} \\
\leq & \int_{a}^{t} \int_{a}^{t}\left(t-u_{1}\right)^{\alpha-1}\left(t-u_{2}\right)^{\alpha-1}\left\|X\left(u_{1}\right)\right\|_{2}\left\|X\left(u_{2}\right)\right\|_{2} \mathrm{~d} u_{1} \mathrm{~d} u_{2} \\
= & \left(\int_{a}^{t}\left(t-u_{1}\right)^{\alpha-1}\left\|X\left(u_{1}\right)\right\|_{2} \mathrm{~d} s\right)\left(\int_{a}^{t}\left(t-u_{2}\right)^{\alpha-1}\left\|X\left(u_{2}\right)\right\|_{2} \mathrm{~d} u_{2}\right) \\
= & \left(\int_{a}^{t}(t-u)^{\alpha-1}\|X(u)\|_{2} \mathrm{~d} u\right)^{2}<+\infty .
\end{aligned}
$$

Notice that in the last step we have used hypothesis (2.15).

Apart from the fractional Riemann-Liouville integral, in the deterministic scenario it is also useful the concept of fractional derivative. In the subsequent development we introduce the definition of the random (left-sided) fractional Caputo derivative, in the m.s. sense. Thus, we firstly give a characterization of its existence, and secondly, a sufficient condition in order to guarantee its existence, in the m.s. sense. 
Chapter 2. Riemann-Liouville and Caputo operators in mean square sense: solving a random linear fractional differential equation

Definition 2.2 Let $\mathcal{D}=[a, b],-\infty<a<b<\infty$, be a finite interval of the real line $\mathbb{R}$. Let $\{X(t): t \in \mathcal{D}\}$ be a 2-SP. The random mean square (left-sided) Caputo fractional derivative of $X(t),\left({ }^{C} D_{a+}^{\alpha} X\right)(t)$, of order $\alpha>0$ is defined by

$$
\left({ }^{C} D_{a+}^{\alpha} X\right)(t):=\left(\mathrm{J}_{a+}^{n-\alpha} X^{(n)}\right)(t)=\frac{1}{\Gamma(n-\alpha)} \int_{a}^{t}(t-u)^{n-\alpha-1} X^{(n)}(u) \mathrm{d} u,
$$

where $n=-[-\alpha]$, being $[\cdot]$ the integer part function and, $X^{(n)}(t)$ denotes the $n$-th m.s. derivative of the 2-SP $X(t)$.

Naturally, the integral that appears in the right-hand side of (2.16) is a m.s. Riemann integral.

Remark 2.3 Analogously to Definition 2.2, the random m.s. (right-sided) Caputo fractional derivative of a $2-S P\{X(t): t \in \mathcal{D}=[a, b]\},-\infty<a<b<\infty$, is defined as

$$
\left({ }^{C} D_{b-}^{\alpha} X\right)(t):=\left(\mathrm{J}_{b-}^{n-\alpha} X^{(n)}\right)(t)=\frac{1}{\Gamma(n-\alpha)} \int_{t}^{b}(u-t)^{n-\alpha-1} X^{(n)}(u) \mathrm{d} u .
$$

Applying Proposition 2.6 to the 2-SP $X^{(n)}(t)$ and using the relationship (2.7), one straightforwardly gets the following characterization on the existence of the random m.s. Caputo fractional derivative of a 2-SP, $X(t)$, that is $n$-times m.s. differentiable.

Proposition 2.8 Let $\{X(t): t \in \mathcal{D}\},-\infty<a<b<\infty$, be a 2-SP n-times differentiable with correlation function $\Gamma_{X}(\cdot, \cdot)$. Then, its (left-sided) Caputo fractional derivative, $\left({ }^{C} D_{a+}^{\alpha} X\right)(t), \alpha>0$, exists in the m.s. sense if, and only if, the following deterministic double Riemann integral

$$
\int_{a}^{t} \int_{a}^{t}\left(t-u_{1}\right)^{n-\alpha-1}\left(t-u_{2}\right)^{n-\alpha-1} \frac{\partial^{2 n} \Gamma_{X}\left(u_{1}, u_{2}\right)}{\partial u_{1}^{n} \partial u_{2}^{n}} \mathrm{~d} u_{1} \mathrm{~d} u_{2}
$$

exists and is finite. 
On the one hand, if we assume that the 2-SP $\{X(t): t \in \mathcal{D}\}$ is $n$-times m.s. differentiable, then applying (2.5) to its $n$-th m.s. derivative, $X^{(n)}(t)$, which is also a 2-SP, one gets

$$
\Gamma_{X^{(n)}}\left(u_{1}, u_{2}\right) \leq\left\|X^{(n)}\left(u_{1}\right)\right\|_{2}\left\|X^{(n)}\left(u_{2}\right)\right\|_{2} .
$$

On the other hand, using an analogous reasoning that was exhibited in the proof of Proposition 2.7 yields

$$
\begin{aligned}
& \int_{a}^{t} \int_{a}^{t}\left|\left(t-u_{1}\right)^{n-\alpha-1}\left(t-u_{2}\right)^{n-\alpha-1} \Gamma_{X^{(n)}}\left(u_{1}, u_{2}\right)\right| \mathrm{d} u_{1} \mathrm{~d} u_{2} \\
\leq & \int_{a}^{t} \int_{a}^{t}\left(t-u_{1}\right)^{n-\alpha-1}\left(t-u_{2}\right)^{n-\alpha-1}\left\|X^{(n)}\left(u_{1}\right)\right\|_{2}\left\|X^{(n)}\left(u_{2}\right)\right\|_{2} \mathrm{~d} u_{1} \mathrm{~d} u_{2} \\
= & \left(\int_{a}^{t}(t-u)^{n-\alpha-1}\left\|X^{(n)}(u)\right\|_{2} \mathrm{~d} u\right)^{2} .
\end{aligned}
$$

Then, the following result has been established:

Proposition 2.9 Let $\alpha>0$ and $\{X(t): t \in \mathcal{D}\}$ be a 2-SP n-times m.s. differentiable such that

$$
\int_{a}^{t}(t-u)^{n-\alpha-1}\left\|X^{(n)}(u)\right\|_{2} \mathrm{~d} u<+\infty .
$$

Then, the random (left-sided) Caputo fractional derivative, $\left({ }^{C} D_{a+}^{\alpha} X\right)(t)$, exists.

Example 2.1 Let $X(t)=A t^{\alpha m}, t \in[0, T], T>0,0<\alpha<1, m>0$, and assume that $A$ is a bounded $R V$ (hence $A \in \mathrm{L}^{2}(\Omega)$ ). Then, $X(t)$ is a 2-SP

$$
\left(\|X(t)\|_{2}\right)^{2}=\mathbb{E}\left[A^{2} t^{2 \alpha m}\right]=t^{2 \alpha m} \mathbb{E}\left[A^{2}\right]<+\infty,
$$

since $\mathrm{E}\left[A^{2}\right]=\left(\|A\|_{2}\right)^{2}<+\infty$. Moreover, $X(t)$ is m.s. differentiable and its m.s. derivative is given by $X^{\prime}(t)=\alpha m A t^{\alpha m-1}$,

$$
\begin{aligned}
& \left(\left\|\frac{X(t+h)-X(t)}{h}-X^{\prime}(t)\right\|_{2}\right)^{2}=\mathbb{E}\left[\left(\frac{A(t+h)^{\alpha m}-A t^{\alpha m}}{h}-\alpha m A t^{\alpha m-1}\right)^{2}\right] \\
& \quad=\mathbb{E}\left[A^{2}\right]\left(\frac{(t+h)^{\alpha m}-t^{\alpha m}}{h}-\alpha m t^{\alpha m-1}\right) \stackrel{h \rightarrow 0}{\longrightarrow} 0,
\end{aligned}
$$


Chapter 2. Riemann-Liouville and Caputo operators in mean square sense: solving a random linear fractional differential equation

since $\mathbb{E}\left[A^{2}\right]<+\infty$ and $g(t)=t^{\alpha m}$ is a deterministic differentiable function whose derivative is $\alpha m t^{\alpha m-1}$. Finally, according to Proposition 2.9 with $n=1, a=0$, we need to check the following deterministic integral

$$
\int_{0}^{t}(t-u)^{-\alpha}\left\|\alpha m A u^{\alpha m-1}\right\|_{2} \mathrm{~d} u=\alpha m\|A\|_{2} \int_{0}^{t}(t-u)^{-\alpha} u^{\alpha m-1} \mathrm{~d} u
$$

is convergent. Since $\|A\|_{2}<+\infty$, it is enough to show that the last integral is finite. To this end, let us make the change of variable: $u=v t$, then using the definition of the beta function (see (2.9)) and its relationship with the gamma function (see (2.10)), one gets

$$
\begin{aligned}
& \int_{0}^{t}(t-u)^{-\alpha} u^{\alpha m-1} \mathrm{~d} u=t^{\alpha(m-1)} \int_{0}^{1} v^{\alpha m-1}(1-v)^{-\alpha} \mathrm{d} v \\
= & t^{\alpha(m-1)} B(\alpha m, 1-\alpha)=t^{\alpha(m-1)} \frac{\Gamma(\alpha m) \Gamma(1-\alpha)}{\Gamma(\alpha(m-1)+1)}<+\infty .
\end{aligned}
$$

Moreover the value of the random m.s. Caputo fractional derivative of $X(t)=A t^{\alpha m}$ is given by

$$
\begin{aligned}
\left({ }^{C} D_{0^{+}}^{\alpha} X\right)(t) & =\frac{1}{\Gamma(1-\alpha)} \int_{0}^{t}(t-u)^{-\alpha} \alpha m A u^{\alpha m-1} \mathrm{~d} u \\
& =\frac{\alpha m A}{\Gamma(1-\alpha)} \int_{0}^{t}(t-u)^{-\alpha} u^{\alpha m-1} \mathrm{~d} u .
\end{aligned}
$$

Observe that the commutation between the m.s. integral and the $R V A$ that we have done in the last step is legitimated because $A$ is a bounded RV (see Proposition 2.1). Finally, substituting expression (2.17) into (2.18) and using property (2.11), one gets the following closed expression for the random (left-sided) Caputo fractional derivative of $X(t)$

$$
\begin{aligned}
\left({ }^{C} D_{0^{+}}^{\alpha} X\right)(t) & =\frac{\alpha m A}{\Gamma(1-\alpha)} t^{\alpha(m-1)} \int_{0}^{1} v^{\alpha m-1}(1-v)^{-\alpha} \mathrm{d} v \\
& =A \frac{\Gamma(\alpha m+1)}{\Gamma(\alpha(m-1)+1)} t^{\alpha(m-1)} .
\end{aligned}
$$




\subsection{Solving the random linear fractional differential equation by the mean square generalized Frobenius method}

This section is devoted to construct a solution SP to the following random fractional linear differential initial value problem (IVP)

$$
\left\{\begin{aligned}
\left({ }^{C} D_{0^{+}}^{\alpha} Y\right)(t)-\lambda Y(t) & =\gamma, \quad t>0, \quad 0<\alpha \leq 1 \\
Y(0) & =\beta_{0}
\end{aligned}\right.
$$

where $\beta_{0}, \lambda$ and $\gamma$ are RVs defined in a common complete probability space $(\Omega, \mathcal{F}, \mathbb{P})$ satisfying certain conditions to be specified later (see hypotheses H1-H2). The solution SP will be constructed by extending the Frobenius method to RFDEs using the random Caputo fractional derivative, that we have previously introduced. The aforementioned extension will be done using the m.s. random calculus.

We will seek a solution SP to the random IVP (2.20) of the form

$$
Y(t)=\sum_{m \geq 0} X_{m} t^{\alpha m}, \quad 0 \leq t \leq T, T>0
$$

imposing that it satisfies the RFDE. Notice that coefficients of series $Y(t)$ have been denoted by $X_{m}$ instead of $Y_{m}$. This fact will be apparent later. As expression (2.21) is a generalized random power series, in order to take advantage of the m.s. random calculus for standard random power series

$$
X(v)=\sum_{m \geq 0} X_{m} v^{m}, \quad 0 \leq v \leq T^{\alpha}, T>0,
$$

let us consider the following expression for the random fractional derivative of the generalized random power series (2.21), in terms of the standard random power series (2.22),

$$
\left({ }^{C} D_{0^{+}}^{\alpha} Y\right)(t)=\left({ }^{C} D_{0^{+}}^{\alpha} X\right)\left(t^{\alpha}\right)=\left(\mathrm{J}_{0^{+}}^{1-\alpha} Z\right)(t)
$$

where $Z \equiv Z(t)=\left(X\left(t^{\alpha}\right)\right)^{\prime}$ denotes the m.s. derivative of the SP $X(t)$ compounded with the deterministic function $t^{\alpha}$. Observe that in agreement with $(2.16)$, the 
Chapter 2. Riemann-Liouville and Caputo operators in mean square sense: solving a random linear fractional differential equation

notation $\left(\mathrm{J}_{0^{+}}^{1-\alpha} Z\right)(t)$ in $(2.23)$ stands for the the Caputo fractional derivative of $Z(t)$ with $a=0$ and $n=1$.

Let us assume that for $t$ fixed in $[0, T]$ the following conditions C1-C2 fulfill:

C1 : $X(v)$, given by $(2.22)$, is a m.s. differentiable at $v=t^{\alpha}$. Moreover,

$$
X^{\prime}\left(t^{\alpha}\right)=\sum_{m \geq 1} m X_{m} t^{\alpha(m-1)} .
$$

C2 : $\frac{\mathrm{d} X(v)}{\mathrm{d} v}$ is m.s. continuous on $v \in\left[0, T^{\alpha}\right]$.

As $0<\alpha \leq 1$, it follows that $\mathcal{V}=\left[0, T^{\alpha}\right]$ contains the range of $g(t)=t^{\alpha}$, i.e., $g\left(\left[a_{1}, a_{2}\right]\right)=g([0, T])=\left[0, T^{\alpha}\right] \subseteq\left[0, T^{\alpha}\right]$. Then, by Theorem $2.2 X(g(t))$ is m.s. differentiable at $t$ and its mean square derivative is given by

$$
Z(t):=Y^{\prime}(t)=\left(X\left(t^{\alpha}\right)\right)^{\prime}=\alpha t^{\alpha-1} X^{\prime}\left(t^{\alpha}\right) .
$$

Therefore, substituting (2.25) into (2.23) and taking into account (2.16), one gets

$$
\begin{aligned}
\left({ }^{C} D_{0^{+}}^{\alpha} Y\right)(t) & =\left(\mathrm{J}_{0^{+}}^{1-\alpha} Z\right)(t)=\frac{1}{\Gamma(1-\alpha)} \int_{0^{+}}^{t}(t-u)^{-\alpha}\left(\alpha u^{\alpha-1} X^{\prime}\left(u^{\alpha}\right)\right) \mathrm{d} u \\
& =\frac{1}{\Gamma(1-\alpha)} \int_{0^{+}}^{t}(t-u)^{-\alpha}\left(\alpha u^{\alpha-1} \sum_{m \geq 1} m X_{m} u^{\alpha(m-1)}\right) \mathrm{d} u \\
& =\frac{1}{\Gamma(1-\alpha)} \int_{0^{+}}^{t}(t-u)^{-\alpha}\left(\sum_{m \geq 1} \alpha m X_{m} u^{\alpha m-1}\right) \mathrm{d} u .
\end{aligned}
$$

We will further assume that the following condition is satisfied

C3 : The random generalized power series $\sum_{m \geq 1} m X_{m} t^{\alpha m-1}$ is m.s. uniformly convergent on the domain $0 \leq t \leq T$. 
Then, the integral and the infinite sum that appear in (2.26) can be commuted, and applying the results shown in Example 2.1, expression (2.26) can be written as

$$
\begin{aligned}
\left({ }^{C} D_{0^{+}}^{\alpha} Y\right)(t) & =\frac{1}{\Gamma(1-\alpha)} \sum_{m \geq 1}\left(\alpha m X_{m} \int_{0^{+}}^{t}(t-u)^{-\alpha} u^{\alpha m-1} \mathrm{~d} u\right) \\
& =\sum_{m \geq 1}\left(X_{m} \frac{\Gamma(\alpha m+1)}{\Gamma(\alpha(m-1)+1)} t^{\alpha(m-1)}\right) \\
& =\sum_{m \geq 0}\left(X_{m+1} \frac{\Gamma(\alpha(m+1)+1)}{\Gamma(\alpha m+1)} t^{\alpha m}\right) .
\end{aligned}
$$

It is important to point out that conditions C1-C3 will be checked once the RVs $X_{m}$, that define the random power series $(2.22)$, are determined for the random IVP (2.20). With this goal and using the Frobenius method, we impose that the random generalized power series solution (2.21) satisfies the RFDE given in (2.20). Substituting formally expressions (2.21) and (2.27) into (2.20), one gets

$$
X_{1} \Gamma(\alpha+1)-\lambda X_{0}-\gamma+\sum_{m \geq 1}\left(X_{m+1} \frac{\Gamma(\alpha(m+1)+1)}{\Gamma(\alpha m+1)}-\lambda X_{m}\right) t^{\alpha m}=0 .
$$

Therefore, a candidate solution SP of the form (2.21) to the random IVP (2.20) can be constructed if the coefficients $X_{m}$ are chosen so that they satisfy the following recurrence

$$
X_{1}=\frac{\lambda \beta_{0}+\gamma}{\Gamma(\alpha+1)}, \quad X_{m+1}=\frac{\lambda \Gamma(\alpha m+1)}{\Gamma(\alpha(m+1)+1)} X_{m}, \quad m \geq 1,
$$

where, we have used that the initial condition is $Y(0)=X_{0}=\beta_{0}$. The recursive application of this relationship yields

$$
X_{m}=\frac{\lambda^{m} \beta_{0}+\lambda^{m-1} \gamma}{\Gamma(\alpha m+1)}, \quad m \geq 1, \quad X_{0}=\beta_{0} .
$$


Chapter 2. Riemann-Liouville and Caputo operators in mean square sense: solving a random linear fractional differential equation

Summarizing, a candidate random generalized power series solution to the IVP (2.20) is given by

$$
Y(t)=X\left(t^{\alpha}\right), \quad X(v)=\sum_{m \geq 0} X_{m, 1} v^{m}+\sum_{m \geq 1} X_{m, 2} v^{m},
$$

where

$$
\left\{\begin{array}{l}
X_{m, 1}=\frac{\lambda^{m} \beta_{0}}{\Gamma(\alpha m+1)}, \\
X_{m, 2}=\frac{\lambda^{m-1} \gamma}{\Gamma(\alpha m+1)},
\end{array}\right.
$$

that is,

$$
Y(t)=\sum_{m \geq 0} \frac{\lambda^{m} \beta_{0}}{\Gamma(\alpha m+1)} t^{\alpha m}+\sum_{m \geq 1} \frac{\lambda^{m-1} \gamma}{\Gamma(\alpha m+1)} t^{\alpha m} .
$$

Remark 2.4 The so-called Mittag-Leffler function

$$
E_{\alpha, \nu}(z)=\sum_{m \geq 0} \frac{z^{m}}{\Gamma(\alpha m+\nu)}, \quad z \in \mathbb{R}, \alpha, \nu \geq 0,
$$

plays a key role in the investigation of deterministic fractional differential equations. Looking at the expression (2.29), which is a random generalized power series, it is suggested a strong connection with the Mittag-Leffler function and the solution found using the random generalized Frobenius technique, namely, $Y(t)=\beta_{0} E_{\alpha, 1}\left(\lambda t^{\alpha}\right)+\gamma t^{\alpha} E_{\alpha, \alpha+1}\left(\lambda t^{\alpha}\right)$. Notice that the study previously performed provides sufficient conditions on the $R V \lambda$ in order to extend the Mittag-Leffler function to the random framework since it is well-defined in the Banach space $\left(\mathrm{L}^{2}(\Omega),\|\cdot\|_{2}\right)$ introduced in $(2.2)$.

So far, we have formally constructed a random generalized power series solution to the random IVP (2.20), which is given by (2.29). Henceforth, we will prove that it is really a rigorous solution by checking that conditions $\mathbf{C 1 - C 3}$ hold. This will be done for a rich enough class of RVs, denoted by $\mathfrak{C}$, that contains significant RVs and that enables us to construct accurate approximations for another important RVs that do not belong to the class $\mathfrak{C}$. These issues will be discussed later. 
Definition 2.3 $A R V, X$, is said to belong to the class $\mathfrak{C}$ if, and only if, there exist positive constants $L>0$ and $H>0$ such that

$$
\mathbb{E}\left[|X|^{m}\right] \leq L H^{m}<+\infty, \quad \forall m \geq 0 .
$$

The inequality (2.31) is derived from the condition that the absolute moments with respect to to the origin of the $X$ growths exponentially.

Remark 2.5 Notice that condition (2.31) is equivalent to

$$
\mathbb{E}\left[|X|^{m}\right]=\mathcal{O}\left(H^{m}\right), \quad H>0, m \geq 0,
$$

where $\mathcal{O}(\cdot)$ stands for the Landau symbol. Observe that every $R V$ of class $\mathfrak{C}$ is a 2-RV.

Remark 2.6 It is important to point out that this class of RVs has already been used successfully to deal with the analysis of some classical (non-fractional) RDEs [42, 31]. As it is shown in [42], bounded RVs belong to the class $\mathfrak{C}$. Hence, relevant RVs such as binomial, hypergeometric, uniform, trapezoidal, beta, $\lambda$-distributed [30], etc., are of class $\mathfrak{C}$. While important unbounded RVs like Poisson, Exponential, Gaussian, etc. can be approximated by truncating appropriately their domain, that is, using bounded RVs.

Now, we are going to legitimate the conditions C1-C3 we have imposed to formally construct the random generalized power solution (2.29). Hereinafter, we will assume the following hypotheses:

H1 : The input data $\beta_{0}, \gamma$ and $\lambda$ are independent 2-RVs.

H2 : $\lambda$ belongs to the class $\mathfrak{C}$ introduced in Definition 2.3.

Observe that hypothesis $\mathbf{H 2}$ entails that

$$
\left\|\beta_{0}\right\|_{2}<+\infty, \quad\|\gamma\|_{2}<+\infty, \quad\left\|\lambda^{m}\right\|_{2}<\sqrt{L} H^{m}<+\infty, \forall m \geq 0,
$$


Chapter 2. Riemann-Liouville and Caputo operators in mean square sense: solving a random linear fractional differential equation

being $L$ and $H$ the positive constants introduced in Definition 2.3. The above bound for $\lambda^{m}$ follows from the definition of the $\|\cdot\|_{2}$-norm given in (2.2) and (2.32)

$$
\left\|\lambda^{m}\right\|_{2}=\sqrt{\mathbb{E}\left[|\lambda|^{2 m}\right]} \leq \sqrt{L H^{2 m}}=\sqrt{L} H^{m}<+\infty, \quad \forall m \geq 0 .
$$

To check condition $\mathbf{C} 1$ we will apply Proposition 2.4 to the two series defined in (2.28). Specifically, for the first series in (2.28) we apply Proposition 2.4 with the following identification: $m_{0}=0, U_{m}(v)=X_{m, 1} v^{m}$. Firstly, we prove that, for each $m \geq 0$ fixed, $X_{m, 1}(v):=X_{m, 1} v^{m}=\left(\lambda^{m} \beta_{0}\right) /(\Gamma(\alpha m+1)) v^{m}$ is m.s. differentiable at $v=t^{\alpha}$, being $X_{m, 1}^{\prime}\left(t^{\alpha}\right)=m \lambda^{m} \beta_{0} v^{\alpha(m-1)} / \Gamma(\alpha m+1)$ its is m.s. derivative. Indeed, observe that for every $v$ such that $0<v \leq T, T>0$, one gets

$$
\begin{aligned}
0 & <\left\|\frac{X_{m, 1}\left(t^{\alpha}+h\right)-X_{m, 1}\left(t^{\alpha}\right)}{h}-X_{m, 1}^{\prime}\left(t^{\alpha}\right)\right\|_{2} \\
& \stackrel{(\mathrm{I})}{=}\left\|\lambda^{m}\right\|_{2}\left\|\beta_{0}\right\|_{2} \frac{1}{\Gamma(\alpha m+1)}\left|\frac{\left(t^{\alpha}+h\right)^{m}-t^{\alpha m}}{h}-m t^{\alpha(m-1)}\right| \\
& \stackrel{\text { (II) }}{\leq} \sqrt{L} H^{m}\left\|\beta_{0}\right\|_{2} \frac{1}{\Gamma(\alpha m+1)}\left|\frac{\left(t^{\alpha}+h\right)^{m}-t^{\alpha m}}{h}-m t^{\alpha(m-1)}\right| \underset{h \rightarrow 0}{\longrightarrow} 0,
\end{aligned}
$$

where in the step (I) we have applied the hypothesis $\mathbf{H} 1$ of statistical independence of RVs $\beta_{0}$ and $\lambda$ together with Proposition 2.2 using the definition of the $\|\cdot\|_{2}$-norm in terms of the expectation operator; in step (II) we have directly used (2.33) and, finally for the last limit we have used that the deterministic function $h(v)=v^{m}$ is differentiable at $v=t^{\alpha}$ and that $\beta_{0}$ is a $2-\mathrm{RV}$, hence $\left\|\beta_{0}\right\|_{2}<+\infty$.

Secondly, we need to prove that for each $m \geq 1$ fixed, $X_{m, 1}^{\prime}(v)=m \lambda^{m} \beta_{0} v^{m-1} / \Gamma(\alpha m+$ 1 ) is m.s. continuous at $v=t^{\alpha}$. This can be checked by following an analogous reasoning to the one exhibited in (2.34).

$$
\begin{aligned}
0 & <\left\|X_{m, 1}^{\prime}\left(t^{\alpha}+h\right)-X_{m, 1}^{\prime}\left(t^{\alpha}\right)\right\|_{2} \\
& =\left\|\lambda^{m}\right\|_{2}\left\|\beta_{0}\right\|_{2} \frac{m}{\Gamma(\alpha m+1)}\left|\left(t^{\alpha}+h\right)^{m-1}-t^{\alpha(m-1)}\right| \\
& \leq \sqrt{L} H^{m}\left\|\beta_{0}\right\|_{2} \frac{\alpha m}{\Gamma(\alpha m+1)}\left|\left(t^{\alpha}+h\right)^{m-1}-t^{\alpha(m-1)}\right| \underset{h \rightarrow 0}{\longrightarrow} 0, \quad t \leq T, T>0,
\end{aligned}
$$


where in the last step we have applied that the deterministic function $v^{m-1}$ is continuous at $t^{\alpha}$. Thirdly, we shall check that the random power series $\sum_{m \geq 0} X_{m, 1}(v)=$ $\sum_{m \geq 0} X_{m, 1} v^{m}$ is m.s. convergent for every $v: 0<v \leq T^{\alpha}$. To do that we will majorize the deterministic series $\sum_{m \geq 0}\left\|X_{m, 1}(v)\right\|_{2}=\sum_{m \geq 0}\left\|X_{m, 1}\right\|_{2} v^{m}$ by a convergent series. With this goal, let us observe that

$$
\begin{aligned}
\left\|X_{m, 1}\right\|_{2} v^{m} & =\left\|\frac{\lambda^{m} \beta_{0}}{\Gamma(\alpha m+1)}\right\|_{2} v^{m} \\
& \leq \sqrt{L} H^{m}\left\|\beta_{0}\right\|_{2} \frac{v^{m}}{\Gamma(\alpha m+1)}:=\delta_{m}(v), \quad 0<v \leq T^{\alpha}, T>0 .
\end{aligned}
$$

Then, using the test ratio for numerical series together with the asymptotic approximation of the gamma function given in (2.12), one gets

$$
\begin{aligned}
\lim _{m \rightarrow+\infty} \frac{\delta_{m+1}(v)}{\delta_{m}(v)} & =H\left(\lim _{m \rightarrow+\infty} \frac{\Gamma(\alpha m+1)}{\Gamma(\alpha(m+1)+1)}\right) v \\
& =H\left(\lim _{m \rightarrow+\infty} \frac{(\alpha m)^{\alpha m} \mathrm{e}^{-\alpha m} \sqrt{2 \pi \alpha m}}{(\alpha(m+1))^{\alpha(m+1)} \mathrm{e}^{-\alpha(m+1)} \sqrt{2 \pi \alpha(m+1)}}\right) v \\
& =H\left(\lim _{m \rightarrow+\infty}\left(\frac{m}{m+1}\right)^{\alpha m} \frac{1}{(\alpha(m+1))^{\alpha}} \sqrt{\frac{m}{m+1}}\right) \mathrm{e}^{\alpha} v \\
& =H\left(\lim _{m \rightarrow+\infty} \frac{1}{(\alpha(m+1))^{\alpha}} \sqrt{\frac{m}{m+1}}\right) v=0,
\end{aligned}
$$

where we have used that

$$
\lim _{m \rightarrow+\infty}\left(\frac{m}{m+1}\right)^{\alpha m}=\lim _{m \rightarrow+\infty}\left(\frac{1}{1+\frac{1}{m}}\right)^{\alpha m}=\lim _{m \rightarrow+\infty}\left(1+\frac{1}{m}\right)^{-\alpha m}=\mathrm{e}^{-\alpha} .
$$

This proves the m.s. convergence of the random power series $\sum_{m \geq 0} X_{m, 1} v^{m}$ defined in (2.28) for every $v$ in $0<v \leq T^{\alpha}$. Fourthly, we shall prove the m.s. uniform convergence of the random power series $\sum_{m \geq 0} X_{m, 1}^{\prime}(v)=\sum_{m \geq 1} m X_{m, 1} v^{m-1}$, being $X_{m, 1}=\lambda^{m} \beta_{0} / \Gamma(\alpha m+1)$ on the domain $0<v \leq T^{\alpha}, T>0$. As the reasoning is analogous to the one exhibited in $(2.35)-(2.36)$, we just show it 
directly

$$
\left\|m X_{m, 1}\right\|_{2} v^{m-1}=m\left\|\frac{\lambda^{m} \beta_{0}}{\Gamma(\alpha m+1)}\right\|_{2} v^{m-1} \leq m \sqrt{L} H^{m}\left\|\beta_{0}\right\|_{2} \frac{T^{m-1}}{\Gamma(\alpha m+1)}:=\hat{\delta}_{m},
$$

and

$$
\lim _{m \rightarrow+\infty} \frac{\hat{\delta}_{m+1}}{\hat{\delta}_{m}}=H\left(\lim _{m \rightarrow+\infty} \frac{1}{(\alpha(m+1))^{\alpha}} \sqrt{\frac{m+1}{m}}\right) T=0 .
$$

All this justifies that the random power series $\sum_{m \geq 0} X_{m, 1} v^{m}$ is m.s. differentiable at $v=t^{\alpha}$. By using similar arguments, one can prove that the second power series $\sum_{m \geq 1} X_{m, 2} t^{m}$ in (2.28) is m.s. differentiable at $v=t^{\alpha}$. Both conclusions allows us to affirm that the random power series $X(v)$, defined in (2.28), satisfies condition C1. As a consequence, by applying Proposition 2.4 the m.s. derivative (2.24) assumed in $\mathbf{C} 1$ is legitimated. Based upon similar arguments, it can be shown that $X(v)$ also satisfies conditions $\mathbf{C 2}$ and $\mathbf{C 3}$.

Summarizing, the following result has been proved.

Theorem 2.3 Let us consider the random fractional linear differential IVP

$$
\left\{\begin{array}{cl}
\left({ }^{C} D_{0^{+}}^{\alpha} Y\right)(t)-\lambda Y(t) & =\gamma, \quad t>0,0<\alpha \leq 1, \\
Y(0) & =\beta_{0},
\end{array}\right.
$$

where the input data satisfy the following hypotheses:

H1: The input data $\beta_{0}, \gamma$ and $\lambda$ are independent RVs.

H2 : The input data $\beta_{0}, \gamma$ and $\lambda$ are 2-RVs, and there exist positive constants $L>0$ and $H>0$ such that

$$
\mathbb{E}\left[|\lambda|^{m}\right] \leq L H^{m}<+\infty, \quad \forall m \geq 0 .
$$

Then,

$$
Y(t)=\sum_{m \geq 0} \frac{\lambda^{m} \beta_{0}}{\Gamma(\alpha m+1)} t^{\alpha m}+\sum_{m \geq 1} \frac{\lambda^{m-1} \gamma}{\Gamma(\alpha m+1)} t^{\alpha m},
$$

is a m.s. solution to the IVP that converges for all $t>0$. 


\subsection{Computing approximations of the mean, the variance, the co- variance and the cross-covariance functions of the solution stochastic process}

So far we have provided sufficient conditions in order to guarantee the m.s. convergence of the solution SP defined by the random generalized power series (2.29). However, from a practical point of view this infinite series needs to be truncated to keep computationally feasible. This motivates the consideration of following finite sum (see (2.28)-(2.29))

$Y_{M}(t)=\sum_{m=0}^{M} X_{m, 1} t^{\alpha m}+\sum_{m=1}^{M} X_{m, 2} t^{\alpha m}=\sum_{m=0}^{M} \frac{\lambda^{m} \beta_{0}}{\Gamma(\alpha m+1)} t^{\alpha m}+\sum_{m=1}^{M} \frac{\lambda^{m-1} \gamma}{\Gamma(\alpha m+1)} t^{\alpha m}$.

From this expression we will compute approximations of both the mean and variance/standard deviation functions of the solution SP given in (2.29). The following property of the m.s. convergence will play a key role to legitimate the computation of approximations.

Proposition 2.10 [109, Th 4.4.3] Let $\left\{X_{M}: M \geq 0\right\}$ and $\left\{Z_{N}: N \geq 0\right\}$ be two sequences of 2-RVs such that $X_{M} \stackrel{[M \rightarrow+\infty]}{m . s .} \longrightarrow X$ and $Z_{N} \stackrel{[N \rightarrow+\infty]}{m . s .} \longrightarrow Z$.

$$
\begin{gathered}
\mathbb{E}\left[X_{M} Z_{N}\right] \underset{M, N \rightarrow+\infty}{\longrightarrow} \mathbb{E}[X Z], \\
\operatorname{Cov}\left[X_{M}, Z_{N}\right] \underset{M, N \rightarrow+\infty}{\longrightarrow} \operatorname{Cov}[X, Z] .
\end{gathered}
$$

Firstly, let us observe that taking $t \in \mathbb{R}$ arbitrary but fixed, and using the following identification $X_{M} \equiv Y_{M}(t)$ for all $M \geq 0$, being $Y_{M}(t)$ the partial sum defined in (2.37) and $Z_{N} \equiv 1$ for all $N \geq 0$ in Proposition 2.10, then one deduces

$$
\mathbb{E}\left[Y_{M}(t)\right] \stackrel{[M \rightarrow+\infty]}{\longrightarrow} \mathbb{E}[Y(t)]
$$

since we have proved the m.s. convergence of $Y_{M}(t)$ for every $t \in \mathbb{R}$. Likewise, applying Proposition 2.10 with $M \equiv N$ and $X_{M}=Z_{N} \equiv Y_{M}(t)$ for all $M, N \geq 0$, being $Y_{M}(t)$ the partial sum defined in (2.37), and taking into account that 
Chapter 2. Riemann-Liouville and Caputo operators in mean square sense: solving a random linear fractional differential equation

$\mathbb{V}\left[Y_{M}(t)\right]=\mathbb{E}\left[\left(Y_{M}(t)\right)^{2}\right]-\left(\mathbb{E}\left[Y_{M}(t)\right]\right)^{2}$ together with (2.38), one gets

$$
\mathbb{V}\left[Y_{M}(t)\right] \stackrel{[M \rightarrow+\infty]}{\longrightarrow} \mathbb{V}[Y(t)] .
$$

Expressions (2.38) and (2.39) legitimize that the approximations $\mathbb{E}\left[Y_{M}(t)\right]$ and $\mathbb{V}\left[Y_{M}(t)\right]$ of the mean and the variance, respectively, constructed by $Y_{M}(t)$ given in (2.37) will converge to the corresponding exact values. At this point, we want to emphasize that this distinctive property of m.s. convergence is what has really justified the use of this strong type of convergence in our study against alternative stochastic convergences like almost surely convergence, convergence in probability and convergence in distribution, which do not have such key property. Below, we shall provide expressions for $\mathbb{E}\left[Y_{M}(t)\right]$ and $\mathbb{V}\left[Y_{M}(t)\right]$. With this goal, let us take the expectation operator and using its linearity property together with the hypothesis H1 of independence for the input RVs $\beta_{0}, \gamma$ and $\lambda$ and Proposition 2.2, one gets:

$$
\mathbb{E}\left[Y_{M}(t)\right]=\mathbb{E}\left[\beta_{0}\right] \sum_{m=0}^{M} \frac{\mathbb{E}\left[\lambda^{m}\right]}{\Gamma(\alpha m+1)} t^{\alpha m}+\mathbb{E}[\gamma] \sum_{m=1}^{M} \frac{\mathbb{E}\left[\lambda^{m-1}\right]}{\Gamma(\alpha m+1)} t^{\alpha m} .
$$

As $\mathbb{V}\left[Y_{M}(t)\right]=\mathbb{E}\left[\left(Y_{M}(t)\right)^{2}\right]-\left(\mathbb{E}\left[Y_{M}(t)\right]\right)^{2}$, in order to compute the variance of (2.37) it is enough to determine an expression of $\mathbb{E}\left[\left(Y_{M}(t)\right)^{2}\right]$ in terms of the statistical moments of the input RVs $\beta_{0}, \gamma$ and $\lambda$. To achieve this goal, let us consider the following development

$$
\begin{aligned}
& \mathbb{E}\left[\left(Y_{M}(t)\right)^{2}\right]=\mathbb{E}\left[\left(\sum_{m=0}^{M} \frac{\lambda^{m} \beta_{0}}{\Gamma(\alpha m+1)} t^{\alpha m}+\sum_{m=1}^{M} \frac{\lambda^{m-1} \gamma}{\Gamma(\alpha m+1)} t^{\alpha m}\right)^{2}\right] \\
& =\mathbb{E}\left[\left(\beta_{0}\right)^{2}\right]\left(\sum_{m=0}^{M} \frac{\mathbb{E}\left[\lambda^{2 m}\right]}{\Gamma^{2}(\alpha m+1)} t^{2 \alpha m}+2 \sum_{m=1}^{M} \sum_{n=0}^{m-1} \frac{\mathbb{E}\left[\lambda^{m+n}\right]}{\Gamma(\alpha m+1) \Gamma(\alpha n+1)} t^{\alpha(m+n)}\right) \\
& +\mathbb{E}\left[\gamma^{2}\right]\left(\sum_{m=1}^{M} \frac{\mathbb{E}\left[\lambda^{2(m-1)}\right]}{\Gamma^{2}(\alpha m+1)} t^{2 \alpha m}+2 \sum_{m=2}^{M} \sum_{n=1}^{m-1} \frac{\mathbb{E}\left[\lambda^{m+n-2}\right]}{\Gamma(\alpha m+1) \Gamma(\alpha n+1)} t^{\alpha(m+n)}\right) \\
& \quad+2 \mathbb{E}\left[\beta_{0}\right] \mathbb{E}[\gamma] \sum_{m=0}^{M} \sum_{n=1}^{M} \frac{\mathbb{E}\left[\lambda^{m+n-1}\right]}{\Gamma(\alpha m+1) \Gamma(\alpha n+1)} t^{\alpha(m+n)},
\end{aligned}
$$


where the hypothesis $\mathbf{H 1}$ has been applied.

If we choose the input RVs $\beta_{0}, \gamma$ and $\lambda$ satisfying the hypotheses $\mathbf{H 1}-\mathbf{H 2}$, then since we have proved the unconditional m.s. convergence over the whole real line of the random generalized power series SP (2.29), it is guaranteed that the approximations of the mean and the variance of the solution $\mathrm{SP}, Y(t)$, to the random IVP (2.20), given by (2.40)-(2.41), will converge to the corresponding exact values for every $t \in \mathbb{R}$.

We finish this section by giving further probabilistic properties of the solution SP, $Y(t)$. These properties will also be constructed from the truncated series (2.37). First, we will calculate an approximation of the cross-covariance function of the solution SP. With this aim let us consider $M, N \geq 1, t, s \in \mathbb{R}$ and the following development based on the properties of the cross-covariance operator together with the expression (2.37)

$$
\begin{aligned}
\mathbb{C}_{Y_{M}, Y_{N}}(t, s)= & \sum_{m=0}^{M} \sum_{n=0}^{N} \operatorname{Cov}\left[X_{m, 1}, X_{n, 1}\right] t^{\alpha m} s^{\alpha n} \\
& +\sum_{m=0}^{M} \sum_{n=1}^{N} \operatorname{Cov}\left[X_{m, 1}, X_{n, 2}\right] t^{\alpha m} s^{\alpha n} \\
& +\sum_{m=1}^{M} \sum_{n=0}^{N} \operatorname{Cov}\left[X_{m, 2}, X_{n, 1}\right] t^{\alpha m} s^{\alpha n} \\
& +\sum_{m=1}^{M} \sum_{n=1}^{N} \mathbb{C o v}\left[X_{m, 2}, X_{n, 2}\right] t^{\alpha m} s^{\alpha n}
\end{aligned}
$$

where each one of the four covariances that appear in the last double sum can be computed in terms of the input data. For example, taking into account (2.28), the hypothesis $\mathbf{H 1}$ of independence of $\mathrm{RVs} \beta_{0}, \gamma$, and $\lambda$, one gets

$$
\operatorname{Cov}\left[X_{m, 1}, X_{n, 1}\right]=\frac{\mathbb{E}\left[\lambda^{m+n}\right] \mathbb{E}\left[\left(\beta_{0}\right)^{2}\right]-\mathbb{E}\left[\lambda^{m}\right] \mathbb{E}\left[\lambda^{n}\right]\left(\mathbb{E}\left[\beta_{0}\right]\right)^{2}}{\Gamma(\alpha m+1) \Gamma(\alpha n+1)}
$$

In an analogous manner, it can be seen that

$$
\operatorname{Cov}\left[X_{m, 1}, X_{n, 2}\right]=\frac{\left(\mathbb{E}\left[\lambda^{m+n-1}\right]-\mathbb{E}\left[\lambda^{m}\right] \mathbb{E}\left[\lambda^{n-1}\right]\right) \mathbb{E}\left[\beta_{0}\right] \mathbb{E}[\gamma]}{\Gamma(\alpha m+1) \Gamma(\alpha n+1)},
$$


Chapter 2. Riemann-Liouville and Caputo operators in mean square sense: solving a random linear fractional differential equation

$$
\operatorname{Cov}\left[X_{m, 2}, X_{n, 1}\right]=\frac{\left(\mathbb{E}\left[\lambda^{m+n-1}\right]-\mathbb{E}\left[\lambda^{m-1}\right] \mathbb{E}\left[\lambda^{n}\right]\right) \mathbb{E}\left[\beta_{0}\right] \mathbb{E}[\gamma]}{\Gamma(\alpha m+1) \Gamma(\alpha n+1)},
$$

and

$$
\operatorname{Cov}\left[X_{m, 2}, X_{n, 2}\right]=\frac{\mathbb{E}\left[\lambda^{m+n-2}\right] \mathbb{E}\left[\gamma^{2}\right]-\mathbb{E}\left[\lambda^{m-1}\right] \mathbb{E}\left[\lambda^{n-1}\right](\mathbb{E}[\gamma])^{2}}{\Gamma(\alpha m+1) \Gamma(\alpha n+1)}
$$

Particularizing the expression $\mathbb{C}_{Y_{M}, Y_{N}}(t, s)$ when

- $s=t$, one obtains the covariance of the random approximations of order $M$ and $N$ at the time instant $t$ of the solution SP.

- $M=N$, one obtains the covariance of the random approximation of order $M$ at the two time instants $t$ and $s$ of the solution SP.

- $M=N$ and $s=t$, one obtains the variance of the random approximation of order $M$ at the time instant $t$ of the solution SP. This expression is equivalent 
to the one determined by (2.40)-(2.41). Specifically,

$$
\begin{aligned}
\mathbb{V}\left[Y_{M}(t)\right]= & \mathbb{E}\left[\left(\beta_{0}\right)^{2}\right] \sum_{m=0}^{M} \sum_{n=0}^{M} \frac{\mathbb{E}\left[\lambda^{m+n}\right]}{\Gamma(\alpha m+1) \Gamma(\alpha n+1)} t^{\alpha(m+n)} \\
& -\left(\mathbb{E}\left[\beta_{0}\right]\right)^{2}\left(\sum_{m=0}^{M} \frac{\mathbb{E}\left[\lambda^{m}\right]}{\Gamma(\alpha m+1)} t^{\alpha m}\right)\left(\sum_{n=0}^{M} \frac{\mathbb{E}\left[\lambda^{n}\right]}{\Gamma(\alpha n+1)} t^{\alpha n}\right) \\
& +\mathbb{E}\left[\beta_{0}\right] \mathbb{E}[\gamma] \sum_{m=0}^{M} \sum_{n=1}^{M} \frac{\left(\mathbb{E}\left[\lambda^{m+n-1}\right]\right)}{\Gamma(\alpha m+1) \Gamma(\alpha n+1)} t^{\alpha(m+n)} \\
& -\mathbb{E}\left[\beta_{0}\right] \mathbb{E}[\gamma]\left(\sum_{m=0}^{M} \frac{\mathbb{E}\left[\lambda^{m}\right]}{\Gamma(\alpha m+1)} t^{\alpha m}\right)\left(\sum_{n=1}^{M} \frac{\mathbb{E}\left[\lambda^{n-1}\right]}{\Gamma(\alpha n+1)} t^{\alpha n}\right) \\
& +\mathbb{E}\left[\beta_{0}\right] \mathbb{E}[\gamma] \sum_{m=1}^{M} \sum_{n=0}^{M} \frac{\mathbb{E}\left[\lambda^{m+n-1}\right]}{\Gamma(\alpha m+1) \Gamma(\alpha n+1)} t^{\alpha(m+n)} \\
& -\mathbb{E}\left[\beta_{0}\right] \mathbb{E}[\gamma]\left(\sum_{m=1}^{M} \frac{\mathbb{E}\left[\lambda^{m-1}\right]}{\Gamma(\alpha m+1)} t^{\alpha m}\right)\left(\sum_{n=0}^{M} \frac{\mathbb{E}\left[\lambda^{n}\right]}{\Gamma(\alpha n+1)} t^{\alpha n}\right) \\
& \left.+\mathbb{E}\left[\gamma^{2}\right] \sum_{m=1}^{M} \sum_{n=1}^{M} \frac{\mathbb{E}\left[\lambda^{m+n-2}\right]}{\Gamma(\alpha m+1) \Gamma(\alpha n+1)} t^{\alpha(m+n)} t_{n=1}^{M} \frac{\mathbb{E}\left[\lambda^{n-1}\right]}{\Gamma(\alpha n+1) t^{n}} t^{\alpha n}\right) \\
& -(\mathbb{E}[\gamma])^{2}\left(\sum_{m=1}^{M} \frac{\mathbb{E}\left[\lambda^{m-1}\right]}{\Gamma(\alpha m+1)} t^{\alpha m}\right)\left(\sum_{n=1}^{M}\right)
\end{aligned}
$$

\subsection{Numerical examples}

This section is devoted to show two examples in order to illustrate all the theoretical results previously established. In particular, through the subsequent examples we want to highlight two key features of our study. Firstly, the method works successfully when $\lambda$ is a RV that belongs to the class $\mathfrak{C}$ introduced in Definition 2.3. Specifically, in the first example (Example 2.2) we will consider that $\lambda$ is a bounded RV, thus it belongs to the class $\mathfrak{C}$ (see Remark 2.6). Secondly, the technique can also be applied to obtain reliable approximations when $\lambda$ is an unbounded RV and it is approximated by an appropriate truncated (thus bounded) RV. This approach is very useful from a practical standpoint since explicit closed 
Chapter 2. Riemann-Liouville and Caputo operators in mean square sense: solving a random linear fractional differential equation

expressions for the statistical absolute moments of many RVs are not available. In such cases, checking condition (2.31) is either very difficult or simply impossible. This issue will be illustrated in the second example (Example 2.3). Additionally, in the Example 2.2 we will further check that the convergence of the mean and the variance (equivalently, the standard deviation) take place over the whole real line, i.e., for every $t \in \mathbb{R}$. Although this fact is already known from the theoretical results previously established, we think that the analysis is very instructive.

Example 2.2 Let us consider the random fractional IVP (2.20) where $\beta_{0}$ and $\gamma$ are 2-RVs such that

$$
\mathbb{E}\left[\beta_{0}\right]=\mathbb{E}[\gamma]=\frac{1}{2}, \quad \mathbb{V}\left[\beta_{0}\right]=\mathbb{V}[\gamma]=\frac{1}{2} .
$$

Observe that, for the sake of generality instead of fixing specific probability distributions for the RVs $\beta_{0}$ and $\gamma$, we have only specified values of their mean and variance. Hence, bounded and unbounded $R V$ s like a uniform $R V$ on the interval $\left[\frac{1-\sqrt{6}}{2}, \frac{1+\sqrt{6}}{2}\right]$; a gamma $R V$ of parameters $\left(r_{1} ; r_{2}\right)=(1 / 2 ; 1)$, and a Gaussian $R V$ of parameters $\left(\mu ; \sigma^{2}\right)=(1 / 2 ; 1 / 2)$, are allowed to play the role of both $R V s$, for example. Furthermore, we will assume that $\lambda$ has a beta distribution of parameters $\left(b_{1} ; b_{2}\right)=(3 / 4 ; 1)$, i.e., $\lambda \sim B e(3 / 4 ; 1)$, hence $\lambda$ is a bounded $R V$ on the interval $[0,1]$ and, as a consequence, it belongs to the class $\mathfrak{C}$ introduced in Definition 2.3 (see Remark 2.6). We will assume that all the input data $\beta_{0}, \gamma$ and $\lambda$ are statistically independent RVs. Therefore, hypotheses $\mathbf{H 1 - H 2}$ hold and it is then guaranteed that the approximation $Y_{M}(t)$, defined in (2.37) via a random generalized power series, will converge in the m.s. sense to the exact solution SP, $Y(t)$. Accordingly, both the mean and the variance (or equivalently, the standard deviation) of the solution SP, $Y(t)$, to the random IVP (2.20) can be approximated using the expressions given in (2.40)-(2.41).

In Figure 2.1, we have plotted the approximations of the mean and the standard deviation of the solution SP to the random IVP (2.20) with $\alpha=0.7$ using different orders of truncations $M \in\{6,7,8,9,10,12,15,17,20\}$. For the sake of clarity in the graphical representation, we have shown the results over two different time intervals $[0,5]$ and $[0,10]$. From these plots, we observe that in order to get better approximations over larger intervals the order of truncation $M$ must be higher. 
It is known from our previous theoretical development (see Section 2.5) that these approximations of order $M$ for the mean and the standard deviation will converge all over the whole real line as $M \rightarrow+\infty$. Nevertheless, it is instructive to check this general result in the context of this example. With this aim, below we shall check this fact. Firstly, let us recall that the explicit value of higher moments of the beta distribution of parameters $\left(b_{1} ; b_{2}\right)$

$$
\mathbb{E}\left[\lambda^{m}\right]=\prod_{n=0}^{m-1} \frac{b_{1}+n}{b_{1}+b_{2}+n}, \quad \lambda \sim B e\left(b_{1} ; b_{2}\right),
$$

satisfies the following first-order recurrence relationship

$$
\mathbb{E}\left[\lambda^{m+1}\right]=\frac{b_{1}+m}{b_{1}+b_{2}+m} \mathbb{E}\left[\lambda^{m}\right]
$$

Let us denote by

$$
e_{m}^{1}(t):=\frac{\mathbb{E}\left[\lambda^{m}\right]}{\Gamma(\alpha m+1)} t^{\alpha m}
$$

the general term of the first deterministic series that defines the approximation of order $M$ for the expectation of the solution SP (see (2.40)). Then, applying the ratio test for numerical series and using (2.44) and (2.36), one gets

$$
\begin{aligned}
\lim _{m \rightarrow+\infty} \frac{e_{m+1}^{1}(t)}{e_{m}^{1}(t)} & =\lim _{m \rightarrow+\infty} \frac{\mathbb{E}\left[\lambda^{m+1}\right]}{\mathbb{E}\left[\lambda^{m}\right]} \frac{\Gamma(\alpha m+1)}{\Gamma(\alpha(m+1)+1)} t^{\alpha} \\
& =\lim _{m \rightarrow+\infty}\left(\frac{b_{1}+m}{b_{1}+b_{2}+m}\right) \lim _{m \rightarrow+\infty}\left(\frac{1}{(\alpha(m+1))^{\alpha}} \sqrt{\frac{m}{m+1}}\right) t^{\alpha}=0
\end{aligned}
$$

for every $t \in \mathbb{R}$ arbitrary but fixed. Following an analogous calculation, it can be seen the second sum in (2.40) converges over the whole real line as $M \rightarrow+\infty$. Observe that the above reasoning proves the convergence of the approximation for the mean given in (2.40) not only for the particular choice $\lambda \sim B e(3 / 4 ; 1)$ but for any values $b_{1}$ and $b_{2}$ of the parameters to the beta distribution.

In order to check the convergence of the approximation of the variance over the whole real line in the context of this example, we will use the representation given in (2.42). Therefore, we must justify the convergence, of the several series that 

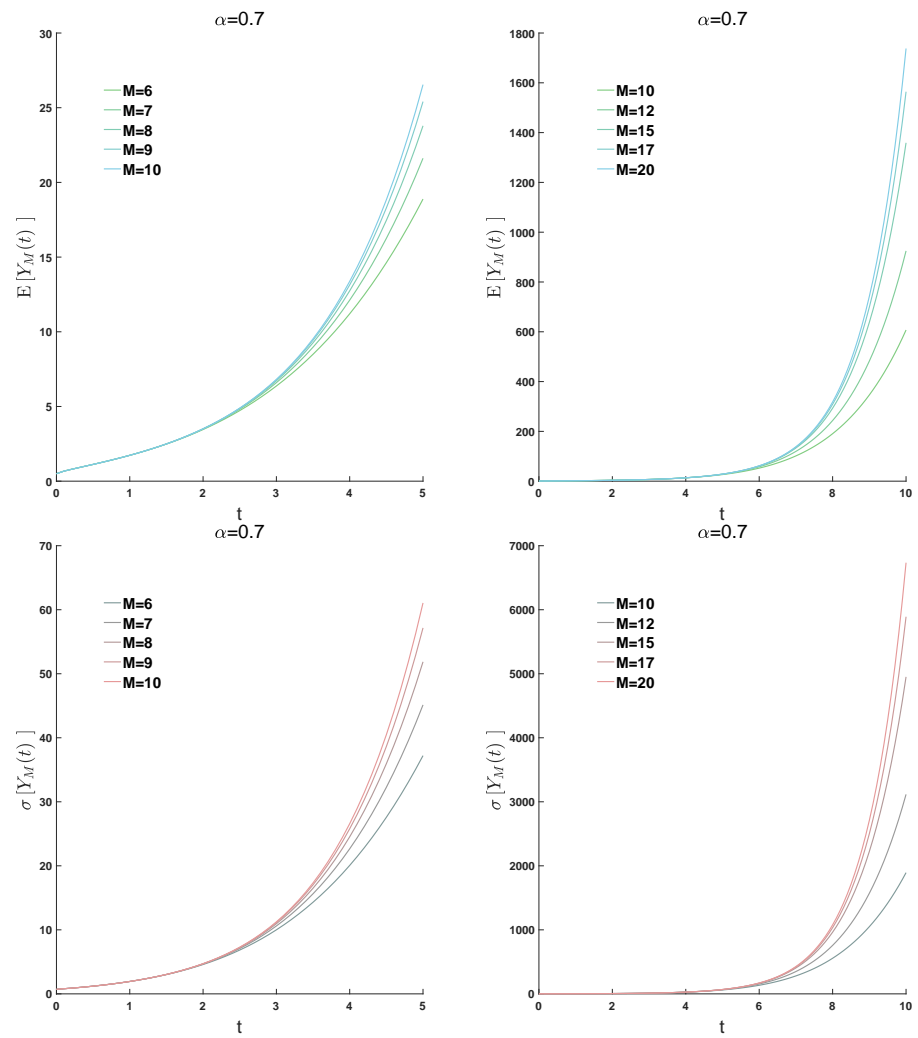

Figure 2.1: Approximations of the mean (top) and the standard deviation (bottom) of the solution SP to the random IVP (2.20) with $\alpha=0.7$ using different orders of truncations $M$ over the time intervals $[0,5]$ and $[0,10]$ in the context of Example 2.2. 
appear in (2.42), for all $t \in \mathbb{R}$. However, essentially there are two different types of such series, namely, single and double series. We shall prove the convergence of the double series by applying Proposition 2.5. Let us define the general term

$$
a_{m n}=\frac{\mathbb{E}\left[\lambda^{m+n}\right]}{\Gamma(\alpha m+1) \Gamma(\alpha n+1)} t^{\alpha(m+n)}, \quad m \geq 0=m_{0}, n \geq 0=n_{0}, t \in \mathbb{R} \text { fixed } .
$$

Since $\lambda$ has a beta distribution, observe that $\mathbb{E}\left[\lambda^{m+n}\right]=\int_{0}^{1} \lambda^{m+n} f_{\lambda}(\lambda) \mathrm{d} \lambda \leq$ $\int_{0}^{1} f_{\lambda}(\lambda) \mathrm{d} \lambda=1$, being $f_{\lambda}(\lambda)$ the PDF of $\lambda$. Then, for $t \in \mathbb{R}$ fixed one gets

$$
\begin{aligned}
\sum_{m=0}^{M} \sum_{n=0}^{N}\left|a_{m n}\right| & =\sum_{m=0}^{M} \sum_{n=0}^{N} \frac{\mathbb{E}\left[\lambda^{m+n}\right]}{\Gamma(\alpha m+1) \Gamma(\alpha n+1)}|t|^{\alpha(m+n)} \\
& \leq \sum_{m=0}^{M} \sum_{n=0}^{N} \frac{|t|^{\alpha m}}{\Gamma(\alpha m+1)} \frac{|t|^{\alpha n}}{\Gamma(\alpha n+1)} \\
& =\left(\sum_{m=0}^{M} \frac{|t|^{\alpha m}}{\Gamma(\alpha m+1)}\right)\left(\sum_{n=0}^{N} \frac{|t|^{\alpha n}}{\Gamma(\alpha n+1)}\right) \\
& \leq\left(\sum_{m \geq 0} \frac{|t|^{\alpha m}}{\Gamma(\alpha m+1)}\right)\left(\sum_{n \geq 0} \frac{|t|^{\alpha n}}{\Gamma(\alpha n+1)}\right) \\
& =\left(E_{\alpha, 1}\left(|t|^{\alpha}\right)\right)^{2}:=\alpha_{0}>0, \quad \forall M \geq m_{0}=0, \forall n \geq n_{0}=0
\end{aligned}
$$

where in the last step we have used (2.30). Therefore, condition (i) of Proposition 2.5 holds. For the symmetry of the general term $a_{m n}$, it is sufficient to check condition (ii) of Proposition 2.5 for the rows, for instance. Let us take $n=\hat{n} \geq 0$ arbitrary but fixed, and let us consider the infinite series

$$
\sum_{m \geq 0} \hat{a}_{m}(t), \quad \hat{a}_{m}(t):=\frac{\mathbb{E}\left[\lambda^{m+\hat{n}}\right]}{\Gamma(\alpha m+1) \Gamma(\alpha \hat{n}+1)}|t|^{\alpha(m+\hat{n})} .
$$

Since

$$
\begin{aligned}
& \lim _{m \rightarrow+\infty} \frac{\hat{a}_{m+1}(t)}{\hat{a}_{m}(t)}=\lim _{m \rightarrow+\infty} \frac{\mathbb{E}\left[\lambda^{m+\hat{n}+1}\right]}{\mathbb{E}\left[\lambda^{m+\hat{n}}\right]} \frac{\Gamma(\alpha m+1) \Gamma(\alpha \hat{n}+1)}{\Gamma(\alpha m+\alpha+1) \Gamma(\alpha \hat{n}+1)} \frac{|t|^{\alpha(m+\hat{n}+1)}}{|t|^{\alpha(m+\hat{n})}} \\
& =\left(\lim _{m \rightarrow+\infty} \frac{b_{1}+m+\hat{n}}{b_{1}+b_{2}+m+\hat{n}}\right)\left(\lim _{m \rightarrow+\infty} \frac{1}{(\alpha(m+1))^{\alpha}} \sqrt{\frac{m}{m+1}}\right)|t|^{\alpha}=0, \quad \forall t \in \mathbb{R},
\end{aligned}
$$


Chapter 2. Riemann-Liouville and Caputo operators in mean square sense: solving a random linear fractional differential equation

where we have used (2.36) and (2.44). The convergence of the second kind of infinite series, which are of the following type $\sum_{m \geq 0}\left(\mathbb{E}\left[\lambda^{m}\right]\right) /(\Gamma(\alpha m+1)) t^{\alpha m}$, follows using the same argument showed in (2.45)-(2.46).

In order to complete the probabilistic description of the solution SP to the fractional IVP (2.20), in Figure 2.2 we have represented the correlation coefficient function of the approximation of order $M$

$$
\rho_{Y_{M}}(t, s)=\frac{\mathbb{C}_{Y_{M}, Y_{M}}(t, s)}{\sqrt{\mathbb{V}\left[Y_{M}(t)\right] \times \sqrt{\mathbb{V}\left[Y_{M}(s)\right]}}} .
$$

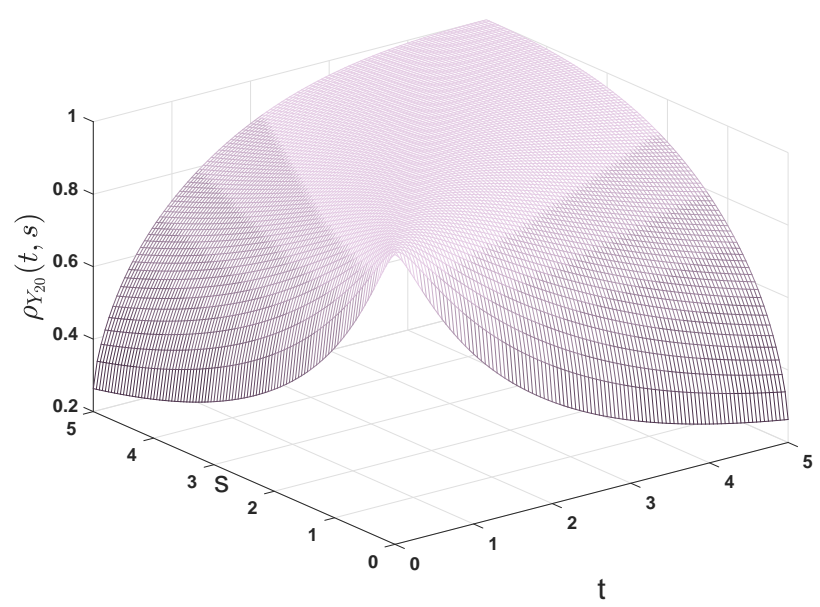

Figure 2.2: Correlation coefficient function $\rho_{Y_{M}}(t, s)$ of the approximation $Y_{M}(t)$ of order $M=20$ of the solution SP $Y(t)$ to the random IVP $(2.20)$ with $\alpha=0.7$ over the time domain $(t, s) \in[0,5] \times[0,5]$ in the context of Example 2.2.

In Figure 2.3, we have represented the approximations of the mean and standard deviation of the solution SP for different values of the differentiation parameters $\alpha=\{0.1,0.2, \ldots, 0.9,1\}$ taking as order of truncation $M=20$ over the time interval $[0,5]$. The plot of the mean provides a nice picture of the manner the solution SP varies as the fractional differentiation parameter changes from 0.1 to 1. It is interesting to observe that the value of $\alpha=1$ corresponds to the classical first derivative. Thus, in that case the plot shows the mean of solution SP to the 
classical random IVP associated to (2.20), i.e.,

$$
\left\{\begin{array}{cl}
Y^{\prime}(t)-\lambda Y(t) & =\gamma, \quad t>0 \\
Y(0) & =\beta_{0} .
\end{array}\right.
$$
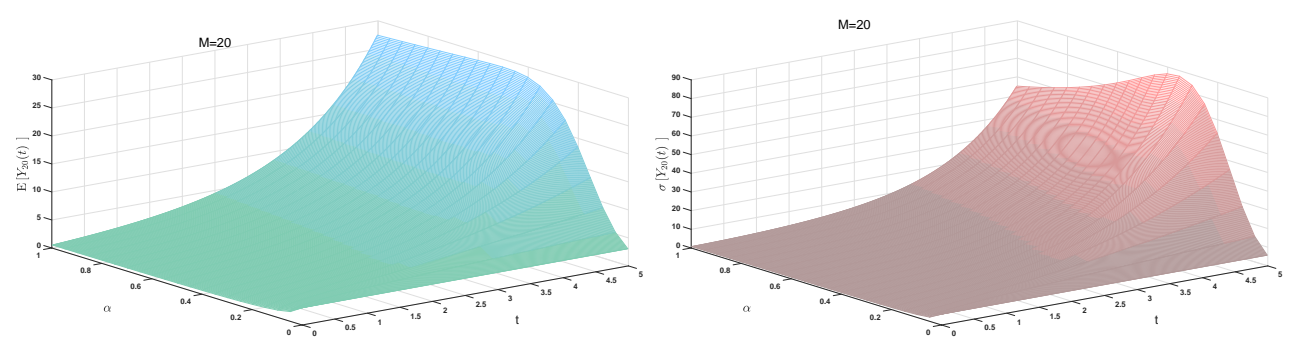

Figure 2.3: Approximations of the mean (top) and the standard deviation (bottom) of the solution SP to the random IVP (2.20) varying the fractional differentiation parameter $\alpha=\{0.1,0.2, \ldots, 0.9,1\}$ taking as order of truncation $M=20$ over the time interval $[0,5]$ in the context of Example 2.2.

We finish this example exhibiting a critical analysis about the computation of the order of truncation $M$ required so that, given an admissible error $\epsilon>0$, the finite numerical series approximation of the mean, given in (2.38), is uniformly bounded by $\epsilon$ in a bounded domain. Our next critical reflection can also be extended to the standard deviation. Let $b_{0}=\left|\mathbb{E}\left[\beta_{0}\right]\right|$ and $c=|\mathbb{E}[\gamma]|$ and assume that

$$
\exists q \in(0,1): H|t|^{\alpha}<q,
$$


Chapter 2. Riemann-Liouville and Caputo operators in mean square sense: solving a random linear fractional differential equation

being $H$ the positive constant associated to the $R V \lambda$, that is assumed to satisfy condition (2.31). Observe that applying (2.38), (2.31) and (2.47), one gets

$$
\begin{aligned}
\left|\mathbb{E}[Y(t)]-\mathbb{E}\left[Y_{M}(t)\right]\right| & =\left|\mathbb{E}\left[\beta_{0}\right] \sum_{m=M+1}^{\infty} \frac{\mathbb{E}\left[\lambda^{m}\right]}{\Gamma(\alpha m+1)} t^{\alpha m}+\mathbb{E}[\gamma] \sum_{m=M+1}^{\infty} \frac{\mathbb{E}\left[\lambda^{m-1}\right]}{\Gamma(\alpha m+1)} t^{\alpha m}\right| \\
& \leq \sum_{m=M+1}^{\infty} \frac{b_{0} \mathbb{E}\left[|\lambda|^{m}\right]+c \mathbb{E}\left[|\lambda|^{m-1}\right]}{\Gamma(\alpha m+1)}|t|^{\alpha m} \\
& \leq \sum_{m=M+1}^{\infty} \frac{b_{0} L H^{m}+c L H^{m-1}}{\Gamma(\alpha m+1)}|t|^{\alpha m} \\
& =\left(b_{0}+\frac{c}{H}\right) L \sum_{m=M+1}^{\infty} \frac{\left(H|t|^{\alpha}\right)^{m}}{\Gamma(\alpha m+1)} \\
& \leq\left(b_{0}+\frac{c}{H}\right) L \sum_{m=M+1}^{\infty} \frac{q^{m}}{\Gamma(\alpha m+1)} \\
& =\left(b_{0}+\frac{c}{H}\right) L \sum_{m=M+1}^{\infty} q^{m}=\left(b_{0}+\frac{c}{H}\right) L \frac{q^{M+1}}{1-q}
\end{aligned}
$$

Therefore, given an admissible error $\epsilon>0$, if we take the order of truncation so that

$$
M \geq\left[(\ln (q))^{-1} \ln \left(\frac{\epsilon(1-q) H}{\left(b_{0} H+c\right) L}\right)-1\right]+1,
$$

where $[\cdot]$ stands for the ceiling function, then it is guaranteed that $\left|\mathbb{E}[Y(t)]-\mathbb{E}\left[Y_{M}(t)\right]\right|<$ $\epsilon, \forall|t|<(q / H)^{1 / \alpha}$. In Table 2.1, we show the theoretical values for the order of truncation $M$ computed by (2.49) taking the same numerical data used to construct the approximations of the mean that we have plotted in Figure 2.1, i.e., $\alpha=0.7$, $b_{0}=c=1 / 2$. Besides, observe that the values $L=H=1$ and $H$ satisfy condition (2.31). Indeed, they can be easily deduced since for the beta $R V \lambda, \mathbb{E}\left[|\lambda|^{m}\right] \leq 1$ for all $m \geq 0$. The figures collected in Table 2.1 have been determined over the domain (2.47) with $q=0.9$, i.e.,

$$
0<|t|<\left(\frac{0.9}{1}\right)^{1 / 0.7}=0.8602648
$$


for different admissible errors $\epsilon>0$. In Table 2.1 we also compare the theoretical values of $M$ with those, denoted by $\hat{M}$, obtained from directed computations. Specifically, $\hat{M}$ has been computed as the first value so that $\left|\mathbb{E}\left[Y_{\hat{M}}(t)\right]-\mathbb{E}\left[Y_{\hat{M}-1}(t)\right]\right|<$ $\epsilon, \forall 0<|t|<0.8602648$, for a given value of $\epsilon>0$. We can observe that the values of $M$ are very conservative estimates. The determination of the order of truncation $M$ given in (2.49) has been based on majorizing the error by a geometric series (see (2.48)). This restricts the analysis to the domain (2.47) (or equivalently to (2.50)), which is contained within the unit interval $(0,1)$. Using appropriate bounds for the remainder of Mittag-Lefller type-series (see (2.30)), $\sum_{m \geq M+1} z^{m} / \Gamma(\alpha m+\nu)$, the above analysis can be carried out for the complementary domain of (2.47). Such appropriate bounds can be found from the results shown in [60, Chapter 4]. Although, interesting from a theoretical standpoint, these results have a limited value in practice, as it has already been pointed out.

\begin{tabular}{|c|c|c|c|c|c|}
\hline & $\epsilon=10^{-3}$ & $\epsilon=10^{-4}$ & $\epsilon=10^{-5}$ & $\epsilon=10^{-6}$ & $\epsilon=10^{-7}$ \\
\hline$M$ & 87 & 109 & 131 & 152 & 174 \\
\hline$\hat{M}$ & 8 & 10 & 11 & 12 & 14 \\
\hline
\end{tabular}

Table 2.1: Theoretical values for the order of truncation $M$ using different values of the admissible error $\epsilon>$ in the context of Example 2.2. These theoretical values are compared with those, denoted by $\hat{M}$, obtained directly from our numerical computations.

Example 2.3 In Remark 2.6 it has been pointed out that the truncation method is a useful technique to approximate unbounded RVs [87, Chapter 5]. In practice, this approach is preferable that checking condition (2.31) for particular probability distributions assigned to the $R V \lambda$. Indeed, this latter idea could be even unaffordable since there are RVs, such as binomial RVs, for which a closed expression for all their statistical moments are not available. Motivated by fact, this example has been devised to illustrate the capability of the proposed method to compute reliable approximations of the mean and the standard deviations of the solution SP to the random IVP (2.20), in the case that the $R V \lambda$ is unbounded but it is approximated by means of appropriate truncation. With this aim, let us assume that $\lambda$ is an exponential $R V$ of mean $1 / \lambda_{0}$, i.e., $\lambda \sim \operatorname{Exp}\left(\lambda_{0}\right)$ and let us consider its probabilistic approximation using the truncation method. We thus approximate the exponential $R V \lambda$ by means of another exponential $R V$, say $\hat{\lambda} \sim \operatorname{Exp}\left(\hat{\lambda}_{0}\right)$, defined on the finite 
Chapter 2. Riemann-Liouville and Caputo operators in mean square sense: solving a random linear fractional differential equation

interval $[0, a], a>0$, so that both $R V s, \lambda$ and $\hat{\lambda}$, have the same mean

$$
\frac{1}{\lambda_{0}}=\mathbb{E}[\lambda]=\mathbb{E}[\hat{\lambda}]
$$

The PDF of $R V \hat{\lambda}$ is

$$
f_{\hat{\lambda}}(\hat{\lambda})=\frac{\hat{\lambda}_{0} \exp \left(-\hat{\lambda}_{0} \hat{\lambda}\right)}{\int_{0}^{a} \hat{\lambda}_{0} \exp \left(-\hat{\lambda}_{0} \hat{\lambda}\right) \mathrm{d} \hat{\lambda}}, \quad 0 \leq \hat{\lambda} \leq a .
$$

Now, we determine the value of the parameter $\hat{\lambda}_{0}$ satisfying condition (2.51)

$$
\frac{1}{\lambda_{0}}=\frac{\int_{0}^{a} \hat{\lambda} \hat{\lambda}_{0} \exp \left(-\hat{\lambda}_{0} \hat{\lambda}\right) \mathrm{d} \hat{\lambda}}{\int_{0}^{a} \hat{\lambda}_{0} \exp \left(-\hat{\lambda}_{0} \hat{\lambda}\right) \mathrm{d} \hat{\lambda}}
$$

In our numerical experiments we have taken $a=10$ and $\lambda_{0}=2$. Thus, according to (2.53) $\lambda_{0}$ is the root of the following nonlinear equation

$$
1-\exp \left(-10 \hat{\lambda_{0}}\right)=\left(1-\exp \left(-10 \hat{\lambda}_{0}\right)\left(1+10 \hat{\lambda}_{0}\right)\right) \hat{\lambda}_{0} .
$$

Using a numerical iterative method it can be checked that $\hat{\lambda}_{0}=1.9999999175537901$ is the solution of (2.54). In order to demonstrate the reliability of the approximations obtained for the mean and the standard deviation using the approach previously described, we have computed the relative error for the mean, RE(Mean), and for the standard deviation, $R E(S D)$. These relative errors have been calculated using the following expressions

$$
\begin{aligned}
& R E(\text { Mean })=R E(\text { Mean })(t ; M)=\frac{\left|\mathbb{E}\left[\hat{Y}_{M}(t)\right]-\mathbb{E}[Y(t)]\right|}{\mathbb{E}[Y(t)]}, \\
& R E(S D)=R E(S D)(t ; M)=\frac{\left|\sqrt{\mathbb{V}\left[\hat{Y}_{M}(t)\right]}-\sqrt{\mathbb{V}[Y(t)]}\right|}{\sqrt{\mathbb{V}[Y(t)]}}
\end{aligned}
$$

where $\mathbb{E}\left[\hat{Y}_{M}(t)\right]$ and $\mathbb{V}\left[\hat{Y}_{M}(t)\right]$ are the approximation of the mean and the variance, respectively, of the solution $S P Y(t)$ at the time point $t$ using the expression (2.40) and (2.42), respectively, with $\alpha=0.7, \mathbb{E}\left[\beta_{0}\right]=\mathbb{E}[\gamma]=0.5$, as in the Example 
2.1 (see (2.43)), and using the bounded $R V, \hat{\lambda} \sim \operatorname{Exp}\left(\hat{\lambda}_{0}=1.9999999175537901\right.$ defined on the finite interval $[0,6]$. Therefore, the higher moments of $\hat{\lambda}$, that appear in (2.40), have been computed by $\mathbb{E}\left[\hat{\lambda}^{m}\right]=\int_{0}^{6} \hat{\lambda}^{m} f_{\hat{\lambda}}(\hat{\lambda}) \mathrm{d} \hat{\lambda}$, being $f_{\hat{\lambda}}(\hat{\lambda})$ defined in (2.52). While the exact mean and variance of $Y(t)$ in (2.55) and (2.56), denoted by $\mathbb{E}[Y(t)]$ and $\mathbb{V}[Y(t)]$, respectively, have been computed using (2.40) and (2.42) by taking $\lambda \sim \operatorname{Exp}(\hat{\lambda}=2)$ and $M=20$, for which the numerical stabilization of approximations has been checked to be exact up to the nine first decimals digits.

In Tables 2.2 and 2.3 we show the numerical results for both relative errors. From the figures collected in these tables we can see that the approximations for the mean and standard deviations obtained using the proposed truncated method are very accurate. As it is expected, these approximations improve as $M$ increases for $t$ fixed, while the accuracy decreases as $t$ departs from the origin for $M$ fixed.

\begin{tabular}{|c|c|c|c|c|c|}
\hline $\mathrm{RE}($ Mean)(t;M) & $t=0.1$ & $t=0.3$ & $t=0.5$ & $t=0.7$ & $t=0.9$ \\
\hline $\mathrm{M}=5$ & $2.7235 \cdot 10^{-5}$ & $2.9732 \cdot 10^{-3}$ & $3.6638 \cdot 10^{-2}$ & $3.1118 \cdot 10^{-1}$ & $8.4798 \cdot 10^{-1}$ \\
\hline $\mathrm{M}=7$ & $1.3773 \cdot 10^{-6}$ & $7.4927 \cdot 10^{-4}$ & $2.0926 \cdot 10^{-2}$ & $2.7211 \cdot 10^{-1}$ & $8.2723 \cdot 10^{-1}$ \\
\hline $\mathrm{M}=10$ & $1.6241 \cdot 10^{-8}$ & $1.2648 \cdot 10^{-4}$ & $1.077 \cdot 10^{-2}$ & $2.2707 \cdot 10^{-1}$ & $7.8983 \cdot 10^{-1}$ \\
\hline $\mathrm{M}=12$ & $3.6711 \cdot 10^{-9}$ & $4.4072 \cdot 10^{-5}$ & $7.2343 \cdot 10^{-3}$ & $1.9819 \cdot 10^{-1}$ & $7.5255 \cdot 10^{-1}$ \\
\hline $\mathrm{M}=15$ & $5.1902 \cdot 10^{-9}$ & $9.9181 \cdot 10^{-6}$ & $3.7974 \cdot 10^{-3}$ & $1.4757 \cdot 10^{-1}$ & $6.4953 \cdot 10^{-1}$ \\
\hline
\end{tabular}

Table 2.2: Relative error for the mean $\mathrm{RE}(\mathrm{Mean})(t ; M)$ computed by $(2.55)$ for different values of $t$ and $M$ in the context of Example 2.3.

\begin{tabular}{|c|c|c|c|c|c|}
\hline $\mathrm{RE}(\mathrm{SD})(\mathrm{t} ; \mathrm{M})$ & $t=0.1$ & $t=0.3$ & $t=0.5$ & $t=0.7$ & $t=0.9$ \\
\hline $\mathrm{M}=5$ & $8.2462 \cdot 10^{-5}$ & $4.3389 \cdot 10^{-1}$ & $9.9813 \cdot 10^{-1}$ & $9.9960 \cdot 10^{-1}$ & $9.9999 \cdot 10^{-1}$ \\
\hline $\mathrm{M}=7$ & $6.4702 \cdot 10^{-6}$ & $3.9683 \cdot 10^{-1}$ & $9.9741 \cdot 10^{-1}$ & $9.9991 \cdot 10^{-1}$ & $9.9999 \cdot 10^{-1}$ \\
\hline $\mathrm{M}=10$ & $1.8467 \cdot 10^{-7}$ & $6.3619 \cdot 10^{-1}$ & $9.9424 \cdot 10^{-1}$ & $9.9953 \cdot 10^{-1}$ & $9.9992 \cdot 10^{-1}$ \\
\hline $\mathrm{M}=12$ & $1.4535 \cdot 10^{-8}$ & $3.8055 \cdot 10^{-1}$ & $9.8742 \cdot 10^{-1}$ & $9.9826 \cdot 10^{-1}$ & $9.9959 \cdot 10^{-1}$ \\
\hline $\mathrm{M}=15$ & $6.0470 \cdot 10^{-9}$ & $3.3668 \cdot 10^{-1}$ & $9.4612 \cdot 10^{-1}$ & $9.8411 \cdot 10^{-1}$ & $9.9352 \cdot 10^{-1}$ \\
\hline
\end{tabular}

Table 2.3: Relative error for the standard deviation $\operatorname{RE}(\mathrm{SD})(t ; M)$ computed by $(2.56)$ for different values of $t$ and $M$ in the context of Example 2.3.

Finally, in the context of this example it is worthy to point out that there is an alternative way to truncate the r.v. $\lambda \sim \operatorname{Exp}\left(\lambda_{0}\right)$. Namely, instead of imposing that the expectations of $\lambda$ and of its approximation $\hat{\lambda}$ match, an alternative manner to truncate $\lambda$ is to take an exponential r.v. defined on a bounded interval, say $\mathcal{I}$, but keeping both r.v.'s $\lambda$ and $\hat{\lambda}$ the same parameter $\lambda_{0}$, so that the length of $\mathcal{I}$ be large enough to guarantee the probability on $\mathcal{I}$ be close to 1 (the total probability). 
Chapter 2. Riemann-Liouville and Caputo operators in mean square sense: solving a random linear fractional differential equation

This approach has been used in example 13 of [42]. Although this is an alternative manner to truncate the r.v. $\lambda \sim \operatorname{Exp}\left(\lambda_{0}\right)$, in the context of our example we have checked that both approaches lead to similar numerical results.

\subsection{Conclusions}

In the first part of this chapter we have extended to the random framework the deterministic Riemann-Liouville integral and Caputo derivative. This extension has been done in the Banach space $\left(\mathrm{L}^{2}(\Omega),\|\cdot\|_{2}\right)$ of the RVs and SPs of secondorder, i.e., having finite variance. This condition is often met for the majority of physical phenomena. An important advantage of the aforementioned extension is that it remains valid for other Banach spaces $\left(\mathrm{L}^{p}(\Omega),\|\cdot\|_{p}\right), p \geq 2$. Furthermore, an additional benefit of our approach is that our results have been established using a strong stochastic convergence, namely the m.s. convergence. Therefore, our results are also valid when using another type of weaker stochastic convergences, such that the convergence in probability and in distribution, which are used in many contexts. In the second part of the chapter, we have taken advantage of the results established in the first part together with a m.s. chain rule for differentiating second-order SPs, to construct a solution SP of the general random linear fractional differential equation assuming mild conditions of the random inputs (initial condition, forcing term and diffusion coefficient). Furthermore, we have given general explicit expressions for constructing reliable approximations of the mean, variance and covariance of the solution SP. Finally, we have illustrated our main theoretical findings and the potentiality of our approach through two examples.

\section{Chapter published}

The results of this chapter have been published in [24]. With regard to this paper, the $\mathrm{PhD}$ candidate has contributed by working in its complete development with more emphasis on the theoretical results (definition of random fractional operators, construction of a convergent solution) and preparing the numerical examples. 


\section{Chapter}

\section{Solving high order random mean square fractional linear differential equations by generalized power series: analysis and computing}

This chapter extends, in two directions, the results presented in the previous chapter. In Chapter 2, a mean square random generalized power series solution has been constructed in the case that the order of the Caputo derivative lies on the

interval ]0,1] and assuming that the diffusion coefficient belongs to a class, $\mathfrak{C}$. However, significant families of unbounded random variables, such as Gaussian and Exponential, for example, do not fall into class $\mathfrak{C}$. Now, in this contribution we first enlarge the class of random variables to which the diffusion coefficient belongs and we prove that the constructed random generalized power series solution is mean square convergent too. Secondly, we construct a mean square random generalized power series solution in the case that $\alpha$ parameter lies on the larger interval ]0,2]. It is particularly enlightening, the numerical study of the convergence of the approximations to the mean and the standard deviation of the solution stochastic process in terms of $\alpha$ parameter and on the type of the probability distribution chosen for the diffusion coefficient. 


\subsection{Introduccion}

In this chapter we deal with the following random fractional initial value problem (IVP)

$$
\left\{\begin{array}{cl}
\left({ }^{C} D_{0^{+}}^{\alpha} Y\right)(t)-\lambda Y(t) & =\gamma, \quad t>0, \quad 0<\alpha \leq 2, \\
Y^{(j)}(0) & =\beta_{j}, \quad 0 \leq j \leq-[-\alpha]-1, j \in \mathbb{N}
\end{array}\right.
$$

where $\mathbb{N}$ and [.] denote the set of positive integers and the integer part function, respectively and $\left({ }^{C} D_{0^{+}}^{\alpha} Y\right)(t)$ denotes the fractional Caputo derivative of order $\alpha$ described in (2.16). Observe that IVP (3.1) refers to two different IVPs by a compact notation. If $\alpha \in] 0,1]$, the IVP (3.1) just has got the initial condition $Y(0)=\beta_{0}$ and it corresponds to the same $\operatorname{IVP}(2.20)$. If $\left.\left.\alpha \in\right] 1,2\right]$, the $\operatorname{IVP}(3.1)$ has got two initial conditions, $Y(0)=\beta_{0}$ and $Y^{\prime}(0)=\beta_{1}$. Henceforth, we will assume that input data $\gamma$ and $\lambda$ are independent real 2-RVs.

In the previous Chapter, we have constructed a m.s. convergent random generalized power series solution to the random IVP (3.1) in the case that the order of the fractional derivative lies in $0<\alpha \leq 1$ (IVP (2.20)). Taking advantage of the key results described in equations (2.38) and (2.39) related to m.s. convergence, approximations of the mean, $\mathbb{E}[Y(t)]$, and of the variance, $\mathbb{V}[Y(t)]$, of the solution SP $Y(t)$ are also computed. These results were established assuming the conditions $\mathbf{H 1}$ and $\mathbf{H 2}$ described in Chapter 2 (page 25). The set of RVs satisfying $\mathbf{H 2}$ are said to make up the class $\mathfrak{C}$ described in Definition 2.3.

As it is indicated in Remark 2.6 of Chapter 2, hypothesis $\mathbf{H 2}$ is fulfilled for bounded RVs. Hence, the results established in Chapter 2 are applicable when the role of random input $\lambda$ is played by RVs such as, Binomial, Hypergeometric, Uniform, Trapezoidal, Beta, etc. Unfortunately, important unbounded RVs, such as Poisson, Exponential, or Gaussian RVs fail to satisfy hypothesis H2. To overcome this drawback, in Chapter 2 one proposes to use the so-called truncation technique [87, Chapter 5]. This approach permits to approximate unbounded RVs, say $X$ by bounded RVs, $\hat{X}$, resulting from the truncation of $X$. In this manner, RV $\hat{X}$ is bounded and thereby hypothesis $\mathbf{H 2}$ is met. Nevertheless, if in the random fractional IVP (3.1) $\lambda$ input is an unbounded RV, say a mean-zero Gaussian RV 
with arbitrary variance $\sigma^{2}>0$, then under the approach proposed in Remark 2.6, the original problem is not really addressed but approximating. As a consequence, approximation errors coming from the truncation procedure are introduced. Motivated by the previous exposition, in this chapter we improve the results established in Chapter 2. First, we will study the random general linear fractional differential equation in the case that the order of the fractional derivative $\alpha$ lies on the larger interval ] 0,2$]$ instead of assuming that $\alpha \in] 0,1]$. We point out that if $\alpha$ lies on an interval of the form $1<\alpha \leq 2$, then two initial conditions must be handled and the construction of the random generalized power series requires a more intricate process. Secondly, we will propose an alternative condition to hypothesis $\mathbf{H 2}$ (page 25 ), which involves the $\lambda$ random input. As it shall be seen later, this new condition permits the consideration of important unbounded RVs, such that Gaussian and Exponential, avoiding the introduction of errors coming from the application of truncation technique. Furthermore, it shall be demonstrated that the random generalized power series (3.15) is still m.s. convergent under our new hypotheses. Then according to equations (2.38) and (2.39), expressions (2.40) and (2.42) can be applied to compute reliable approximations for both the mean and the variance of the solution SP $Y(t)$ to the random fractional IVP (3.1) with $\alpha_{0}=1$. Additionally, it is important to stress that the new condition is also satisfied by bounded RVs, thus the results established in Chapter 2 are fully retained in this Chapter.

The chapter is organized as follows. In Section 3.2 we introduce a class of RVs that will play the role of diffusion coefficient $\lambda$ in the random IVP (3.1). By means of different examples, we show that this class contains all bounded RVs and significant unbounded RVs as well. The solution SP to random IVP (3.1) is constructed by a random generalized power series whose m.s. convergence is studied in Section 3.3. This analysis is divided in two cases depending of the order of the fractional derivative $\alpha$ : Case I corresponds to $\alpha \in] 0,1]$ while Case II deals with $\alpha \in] 1,2$ ]. In Section 3.4 we show several examples where our main theoretical findings are illustrated. Conclusions are drawn in Section 3.5. 


\subsection{Introducing a key class of random variables}

In the next section, we shall construct a random generalized power series solution to IVP (3.1). This section is devoted to introduce a class of RVs that will allow us to enlarge, with respect to the previous Chapter 2, the family of input data playing the role of the diffusion coefficient $\lambda$ in the IVP (3.1) for which the random generalized power series solution is m.s. convergent.

Hereinafter we will assume that $\lambda$ is a second-order RV such that

$$
\exists \eta, \mathcal{H}>0, p \geq 0:\left\|\lambda^{m}\right\|_{2} \leq \eta \mathcal{H}^{m-1}((m-1) !)^{p}, \quad \forall m \geq m_{0} \geq 1, \quad m, m_{0} \in \mathbb{Z}^{+} .
$$

The class of all RVs satisfying condition (3.2) will be denoted by $\hat{\mathfrak{C}}$. Observe that the latter condition contains as a particular case condition $\mathbf{H 2}$ of Chapter 2, since it is obtained by taking $p=0$ and $\eta=L / \mathcal{H}>0$ in (3.2), i.e., $\mathfrak{C} \subset \hat{\mathfrak{C}}$. As a consequence, the results that will be presented in this chapter generalize the ones given in Chapter 2.

As it will be seen later, condition (3.2) is very useful to prove the m.s. convergence of the random generalized power series to be constructed, however it may not be easy to check whether it is satisfied by specific families of RVs. This is the reason why we now introduce the following condition (3.3) that, in practice, is easier to check than (3.2) and, as it will be shown below, it entails condition (3.2). Motivated by this fact, let us assume that $\lambda$ is a second-order RV such that

$$
\exists p \geq 0: \frac{\left\|\lambda^{m+1}\right\|_{2}}{\left\|\lambda^{m}\right\|_{2}}=\mathcal{O}\left(m^{p}\right), \quad \forall m: m \geq m_{0} \geq 1, \quad m, m_{0} \text { integers },
$$

where $\mathcal{O}(\cdot)$ denotes the Landau's symbol. By definition of $\mathcal{O}(\cdot)$, condition (3.3) means

$$
\exists \mathcal{H}, p \geq 0:\left\|\lambda^{m+1}\right\|_{2} \leq \mathcal{H} m^{p}\left\|\lambda^{m}\right\|_{2}, \quad \forall m: m \geq m_{0} \geq 1, \quad m, m_{0} \text { integers. }
$$

Observe that it is sufficient this inequality to be fulfilled for $m_{0}$ large enough. As $\lambda \in \mathrm{L}^{2}(\Omega),\|\lambda\|_{2}<+\infty$ and let $\eta$ be a finite positive number so that $\eta \geq\|\lambda\|_{2}$. Without loss of generality, hereinafter let us assume that $m_{0}=1$. Then, using a 
recursive argument in (3.4) one gets

$$
\begin{aligned}
\left\|\lambda^{m+1}\right\|_{2} & \leq \mathcal{H} m^{p}\left\|\lambda^{m}\right\|_{2} \\
& \leq \mathcal{H}^{2}(m(m-1))^{p}\left\|\lambda^{m-1}\right\|_{2} \\
& \leq \mathcal{H}^{3}(m(m-1)(m-2))^{p}\left\|\lambda^{m-2}\right\|_{2} \\
& \vdots \\
& \leq \eta \mathcal{H}^{m}(m !)^{p}, \quad \forall m \geq 1 \text { integer. }
\end{aligned}
$$

Summarizing, condition (3.3) (or equivalently, (3.4)) entails

H2: The moments of RV $\lambda$ satisfy

$$
\exists \eta, \mathcal{H}>0, p \geq 0:\left\|\lambda^{m}\right\|_{2} \leq \eta \mathcal{H}^{m-1}((m-1) !)^{p}, \quad \forall m \geq 1 \text { integer }
$$

being $\eta \geq\|\lambda\|_{2}$ finite. Now, we introduce important families of RVs satisfying condition (3.3) (or equivalently (3.4)) and hence condition (3.5) too.

Example 3.1 Let $\lambda$ be a bounded $R V$. Then there exist real constants $l_{1}$ and $l_{2}$ with $l_{1}<l_{2}$ such that $\mathbb{P}\left[\left\{\omega \in \Omega: l_{1}(\omega)<\lambda(\omega) \leq l_{2}(\omega)\right\}\right]=1$. Observe that clearly $\lambda$ is a second-order $R V$, i.e. $\lambda \in \mathrm{L}^{2}(\Omega)$. Let us assume, without loss of generality, that $\lambda$ is an absolutely continuous $R V$ being $f_{\lambda}(\lambda)$ its probability density function. If $\hat{l}=\max \left\{1,\left|l_{1}\right|,\left|l_{2}\right|\right\} \geq 1$, then

$$
\left\|\lambda^{m}\right\|_{2}=\left(\mathbb{E}\left[\lambda^{2 m}\right]\right)^{1 / 2}=\left(\int_{l_{1}}^{l_{2}} \lambda^{2 m} f_{\lambda}(\lambda) \mathrm{d} \lambda\right)^{1 / 2} \leq \hat{l}^{m}\left(\int_{l_{1}}^{l_{2}} f_{\lambda}(\lambda) \mathrm{d} \lambda\right)^{1 / 2}=\hat{l}^{m}
$$

where in the last step we have applied that $\int_{l_{1}}^{l_{2}} f_{\lambda}(\lambda) \mathrm{d} \lambda=1$ since $f_{\lambda}(\lambda)$ is a probability density function. Therefore, (3.5) is satisfied for $\eta=\hat{l}, \mathcal{H}=\hat{l}^{m-1}$ and $p=0$. If $\hat{l}=\max \left\{\left|l_{1}\right|,\left|l_{2}\right|\right\} \leq 1$ instead, it is clear that $\left\|\lambda^{m}\right\|_{2} \leq 1$ and taking $\eta=\mathcal{H}=1$ and $p=0$, condition (3.5) also holds. The previous reasoning is also valid if $\lambda$ is a discrete $R V$. As a consequence, any truncated $R V$ as well as important bounded RVs such as Binomial, Hypergeometric, Uniform, Beta, Triangular, etc., satisfy condition (3.5). 
It is important to point out that any bounded RV satisfies (3.5), so in practice, any unbounded RV can be adequately truncated so that the truncated (hence bounded) RV behaves approximately as the original unbounded RV, that is, approximately preserving its main probabilistic information like mean, variance, etc. A way to do that, which is supported by Markov-Chebyshev type inequalities, is to consider an interval with centre the mean and radius a multiple, say $k>0$, of the standard deviation of the unbounded RV [87, Chapter 5]. Taking $k$ large enough, most of the probability mass is captured and the mean and standard deviation of the original unbounded RV are then approximated. Alternatively, one can construct a truncated parametric distribution preserving a number of statistical moments (mean, variance, etc.) so that the new (bounded) distribution approximates quite well the original unbounded distribution, this approach is based upon the matching moment method [87, Chapter 5]. However, approximation errors coming from the truncation procedure can be introduced.

Example 3.2 Let $\lambda$ be a Gaussian $R V$ with zero mean, $\mu=0$, and arbitrary finite variance, $\sigma^{2}>0$, i.e. $\lambda \sim N\left(0 ; \sigma^{2}\right)$. Hence, $\lambda \in \mathrm{L}^{2}(\Omega)$. It is known that (see [99], for instance)

$$
\mathbb{E}\left[\lambda^{n}\right]=\left\{\begin{array}{cl}
\frac{n !}{2^{\frac{n}{2}}\left(\frac{n}{2}\right) !} \sigma^{n} & \text { if } n \text { is even }, \\
0 & \text { if } n \text { is odd }
\end{array}\right.
$$

then, by the definition of the 2-norm (see (2.2)) one gets

$$
\frac{\left\|\lambda^{m+1}\right\|_{2}}{\left\|\lambda^{m}\right\|_{2}}=\frac{\left(\mathbb{E}\left[\lambda^{2(m+1)}\right]\right)^{1 / 2}}{\left(\mathbb{E}\left[\lambda^{2 m}\right]\right)^{1 / 2}}=\sigma \sqrt{\frac{(2 m+2)(2 m+1)}{2(m+1)}}=\mathcal{O}\left(m^{1 / 2}\right) .
$$

Therefore, condition (3.3) is satisfied for $p=1 / 2$. Following the reasoning exhibited to deduce condition (3.5), it is straightforward to derive that this condition is fulfilled for $\mathcal{H}=\sigma \sqrt{2}, p=1 / 2$ and $\eta=\sigma>0$. 
Example 3.3 Let $\lambda$ be an Exponential $R V$ of parameter, $\nu>0$, i.e. $\lambda \sim \operatorname{Exp}(\nu)$. Hence, $\lambda \in \mathrm{L}^{2}(\Omega)$. It is known that (see [99], for instance)

$$
\mathbb{E}\left[\lambda^{m}\right]=\frac{m !}{\nu^{m}}, \quad m \geq 0,
$$

then

$$
\frac{\left\|\lambda^{m+1}\right\|_{2}}{\left\|\lambda^{m}\right\|_{2}}=\frac{\left(\mathbb{E}\left[\lambda^{2(m+1)}\right]\right)^{1 / 2}}{\left(\mathbb{E}\left[\lambda^{2 m}\right]\right)^{1 / 2}}=\frac{1}{\nu} \sqrt{(2 m+2)(2 m+1)}=\mathcal{O}(m) .
$$

Therefore, condition (3.3) is satisfied for $p=1$. Moreover, condition (3.5) holds for $\mathcal{H}=2 / \nu, p=1$ and $\eta=\sqrt{2} / \nu>0$.

Example 3.4 Let $\lambda$ be a Weibull $R V$ of parameters $a>0$ and $b>0$, i.e. $\lambda \sim$ $W e(a ; b)$. It is known that (see [99], for instance)

$$
\mathbb{E}\left[\lambda^{m}\right]=a^{m} \Gamma\left(1+\frac{m}{b}\right), \quad m \geq 0,
$$

being $\Gamma(\cdot)$ the classical gamma function. Using the definition of the 2-norm and (3.11), one gets

$$
\frac{\left\|\lambda^{m+1}\right\|_{2}}{\left\|\lambda^{m}\right\|_{2}}=\frac{\left(\mathbb{E}\left[\lambda^{2(m+1)}\right]\right)^{1 / 2}}{\left(\mathbb{E}\left[\lambda^{2 m}\right]\right)^{1 / 2}}=a \sqrt{\frac{\Gamma\left(1+\frac{2 m+2}{b}\right)}{\Gamma\left(1+\frac{2 m}{b}\right)}} .
$$

As condition (3.3) must be satisfied for $m \geq m_{0} \geq 1$ integer, then taking $m_{0}$ large enough and using Stirling's formula

$$
\Gamma(x+1) \approx x^{x} \mathrm{e}^{-x} \sqrt{2 \pi x}, \quad x \rightarrow+\infty,
$$


one obtains the following asymptotic relationship

$$
\begin{aligned}
\frac{\Gamma\left(1+\frac{2(m+1)}{b}\right)}{\Gamma\left(1+\frac{2 m}{b}\right)} & \approx \frac{\left(\frac{2(m+1)}{b}\right)^{\frac{2(m+1)}{b}} \mathrm{e}^{-\frac{2(m+1)}{b}} \sqrt{2 \pi \frac{2(m+1)}{b}}}{\left(\frac{2 m}{b}\right)^{\frac{2 m}{b}} \mathrm{e}^{-\frac{2 m}{b}} \sqrt{2 \pi \frac{2 m}{b}}} \\
& \approx\left(\frac{m+1}{m}\right)^{\frac{2 m}{b}}\left(\frac{2(m+1)}{b}\right)^{\frac{2}{b}} e^{-\frac{2}{b}} \sqrt{\frac{m+1}{m}} \\
& \approx\left(\frac{2 m}{b}\right)^{\frac{2}{b}},
\end{aligned}
$$

where in the last step we have used that $\left(\frac{m+1}{m}\right)^{\frac{2 m}{b}} \stackrel{m \rightarrow+\infty}{\longrightarrow} \mathrm{e}^{\frac{2}{b}}$. Then, substituting (3.14) in (3.12) one deduces

$$
\frac{\left\|\lambda^{m+1}\right\|_{2}}{\left\|\lambda^{m}\right\|_{2}} \approx a \sqrt{\left(\frac{2 m}{b}\right)^{2 / b}}=\mathcal{O}\left(m^{\frac{1}{b}}\right) .
$$

As a consequence, $\lambda \sim W e(a ; b)$ satisfies condition $(3.3)$ for $p=1 / b>0$ and also, condition (3.5) and (3.4) are satisfied taking $\mathcal{H}=a(2 / b)^{1 / b}$ and $\eta=a \sqrt{\Gamma(1+2 / b)}$.

In Table 3.1, we collect the families of RVs that satisfy the inequality (3.5) described in Examples 3.1, 3.2, 3.3 and 3.4 indicating the value of involved parameters $p, \mathcal{H}$ and $\eta$.

Table 3.1: Some important families of RVs that satisfy inequality (3.5).

\begin{tabular}{lccc}
\hline Distribution & $p$ & $\mathcal{H}$ & $\eta$ \\
\hline \hline Bounded & 0 & 1 & 1 \\
\hline Gaussian $\sim \mathrm{N}\left(0 ; \sigma^{2}\right)$ & $1 / 2$ & $\sigma \sqrt{2}$ & $\sigma$ \\
\hline Exponential $\sim \operatorname{Exp}(\lambda)$ & 1 & $2 / \lambda$ & $\sqrt{2} / \lambda$ \\
\hline Weibull $\sim \operatorname{We}(a ; b)$ & $1 / b$ & $a(2 / b)^{1 / b}$ & $a \sqrt{\Gamma(1+2 / b)}$ \\
\hline
\end{tabular}




\subsection{Mean square convergence of the random generalized power series solution}

This section is devoted to construct a random generalized power series solution to the IVP (3.1) and then proving its m.s. convergence. Finally, we will give closed-form expressions for the approximations of the mean, the variance and the covariance functions of the solution SP.

The analysis will be split in two cases: Case I corresponding to $0<\alpha \leq 1$ and Case II corresponding to $1<\alpha \leq 2$. The former is strongly related to the results established in Chapter 2, hence it will be discussed taking advantage of such previous findings. In particular, as the representation of the solution SP is just the one shown in Chapter 2, here we will focus on the analysis of the m.s. convergence assuming that the diffusion coefficient $\lambda$ satisfies condition $\hat{\mathbf{H}} \mathbf{2}$ (see expression (3.2)) instead of $\mathbf{H 2}$, described in Chapter 2 (page 25). As Case II involves the two RVs $\beta_{0}$ and $\beta_{1}$ through initial conditions, it will be assumed the following hypothesis:

$\hat{\mathbf{H}} 1$ : Inputs $\beta_{0}, \beta_{1}, \gamma$ and $\lambda$ are (mutually) independent second-order RVs,

instead of $\mathbf{H 1}$. As it shall be seen later, the study of Case II will require further analysis.

\subsubsection{Case I: $0<\alpha \leq 1$}

In accordance with Chapter 2, it is known that the solution SP to the random fractional IVP (3.1) $0<\alpha \leq 1$ is given by

$$
Y(t)=\sum_{m=0}^{+\infty} \frac{\lambda^{m} \beta_{0}}{\Gamma(\alpha m+1)} t^{\alpha m}+\sum_{m=1}^{+\infty} \frac{\lambda^{m-1} \gamma}{\Gamma(\alpha m+1)} t^{\alpha m} .
$$

In this section we will establish sufficient conditions in order to guarantee the m.s. convergence of this random generalized power series assuming that input data $\beta_{0}$, $\gamma$ and $\lambda$ satisfy hypotheses $\mathbf{H} \mathbf{1}$ and $\hat{\mathbf{H}} \mathbf{2}$. This will be done just for the first series in (3.15), since the proof for the second series can be done analogously. 
Let us observe that for $0<\alpha \leq 1$ and $t>0$ one gets

$$
\left\|\frac{\lambda^{m} \beta_{0}}{\Gamma(\alpha m+1)} t^{\alpha m}\right\|_{2}=\frac{\left\|\lambda^{m}\right\|_{2}\left\|\beta_{0}\right\|_{2}}{\Gamma(\alpha m+1)} t^{\alpha m} \leq \frac{\eta H^{m-1}((m-1) !)^{p}\left\|\beta_{0}\right\|_{2}}{\Gamma(\alpha m+1)} t^{\alpha m}=: \delta_{m}(t),
$$

where probabilistic independence between $\mathrm{RVs} \lambda^{m}$ and $\beta_{0}$ (justified by hypothesis $\mathbf{H 1}$ and Proposition 2.2, see also [63, page 92]) and hypothesis $\hat{\mathbf{H}} \mathbf{2}$ have been applied. Down below, we obtain sufficient conditions for the m.s. absolute convergence of first series in (3.15) using the D'Alembert or ratio test

$$
\begin{aligned}
\lim _{m \rightarrow+\infty} \frac{\delta_{m+1}(t)}{\delta_{m}(t)} & =\mathcal{H} t^{\alpha} \lim _{m \rightarrow+\infty}\left(m^{p} \frac{\Gamma(\alpha m+1)}{\Gamma(\alpha(m+1)+1)}\right) \\
& =\mathcal{H}\left(\frac{t}{\alpha}\right)^{\alpha} \lim _{m \rightarrow+\infty} \frac{m^{p}}{(m+1)^{\alpha}} \\
& =\left\{\begin{array}{cl}
0 & \text { if } 0 \leq p<\alpha, \forall t>0, \\
\mathcal{H}\left(\frac{t}{\alpha}\right)^{\alpha} & \text { if } 0 \leq p=\alpha, \forall t>0 .
\end{array}\right.
\end{aligned}
$$

Observe that in the second earlier step we have used the Stirling's formula (3.13) to conclude

$$
\begin{aligned}
\lim _{m \rightarrow+\infty} \frac{\Gamma(\alpha m+1)}{\Gamma(\alpha(m+1)+1)} & =\lim _{m \rightarrow+\infty} \frac{(\alpha m)^{\alpha m} \mathrm{e}^{-\alpha m} \sqrt{2 \pi \alpha m}}{(\alpha(m+1))^{\alpha(m+1)} \mathrm{e}^{-\alpha(m+1)} \sqrt{2 \pi \alpha(m+1)}} \\
& =\frac{1}{\alpha^{\alpha}} \lim _{m \rightarrow+\infty} \frac{1}{(m+1)^{\alpha}} .
\end{aligned}
$$

As a consequence of the previous development together with equations (2.38) and (2.39), the following result has been established

Theorem 3.1 Let us consider the random fractional IVP (3.1) with $0<\alpha \leq 1$ and assume that the inputs data $\beta_{0}, \gamma$ and $\lambda$ are RVs satisfying hypotheses $\mathbf{H 1}$ and $\hat{\mathbf{H} 2}$. If $p \geq 0$ and $\alpha \in] 0,1]$ are so that $p<\alpha$, then the random generalized power series $Y(t)$ given by (3.15) is a m.s. solution to the IVP (3.1) for all $t \geq 0$. While, if $p=\alpha$, then $Y(t)$ is a m.s. solution to the IVP (3.1) over the domain $t: 0 \leq t<\alpha / \mathcal{H}^{\frac{1}{\alpha}}$. Furthermore, the approximations of the mean and the variance (or standard deviation) given by (2.40) and (2.42) will also converge at least over the domains previously specified for the m.s. convergence. 
Remark 3.1 The rigorous construction of solution SP (3.15) would require to check some technical hypotheses. This analysis has been omitted here because it follows an analogous development to one exhibited in Chapter 2, but using the new hypothesis $\hat{\mathbf{H}} \mathbf{2}$ for $\lambda$ instead of $\mathbf{H 2}$.

Remark 3.2 The above result provides sufficient conditions to guarantee the m.s. convergence of the random generalized power series solution (3.15) to the random fractional IVP (3.1) assuming mild hypotheses that include a wide range of $R V$, namely all bounded RVs and significant unbounded RVs such as Gaussian and Weibull, for instance. It is interesting to observe that our m.s. convergence analysis depends on parameter $p$ associated to the diffusion $R V \lambda$ (see expression (3.5)) and on the order of the fractional derivative $\alpha \in] 0,1]$. In Theorem 3.1 we have shown that the random generalized power series (3.15) is m.s. unconditionally convergent for all $t \geq 0$ provided $p<\alpha$, while the domain of m.s. convergence becomes smaller when $p=\alpha$, specifically $t: 0 \leq t<\alpha / \mathcal{H}^{\frac{1}{\alpha}}$. Thus, in this latter case the domain depends on both the constant $\mathcal{H}$ associated to hypothesis $\hat{\mathbf{H} 2}$ (see expression (3.5)) and on the order of the fractional derivative $\alpha \in] 0,1]$. This issue will be analyzed deeper throughout the examples exhibited in the next section.

\subsubsection{Case II: $1<\alpha \leq 2$}

This section is devoted to construct a solution SP to random IVP (3.1) when $1<\alpha \leq 2$. This solution is then constructed by means of a random generalized power series. We will prove the m.s. convergence of this series under mild conditions. Finally, we will provide approximations of the mean, the variance, the covariance and the cross-covariance function of the solution SP.

The solution SP will be sought by combining the random Frobenius method and a m.s. chain rule for differentiating second-order SPes, that has been established in [46]. As our subsequent development follows in broad outline that of ideas exhibited in Chapter 2, it will be presented in a direct manner. The solution SP 
$Y(t)$ will be constructed in the following form

$$
Y(t)=Y_{1}(t)+Y_{2}(t), \quad\left\{\begin{array}{l}
Y_{1}(t)=\sum_{m \geq 0} X_{m} t^{\alpha m}, \\
Y_{2}(t)=\sum_{m \geq 0}^{m} Y_{m} t^{\alpha m+1} .
\end{array}\right.
$$

In order to apply Frobenius method, first we need to obtain the m.s. Caputo derivative of order $\alpha$ to $Y_{1}(t)$ and $Y_{2}(t)$. To this end, we define $\hat{Y}_{1}(t)=\sum_{m \geq 0} X_{m} t^{m}$, hence $Y_{1}(t)=\hat{Y}_{1}\left(t^{\alpha}\right)$. According to Chapter 2, the random m.s. Caputo derivative is given by

$$
\left({ }^{C} D_{0^{+}}^{\alpha} Y_{1}\right)(t)=\left({ }^{C} D_{0^{+}}^{\alpha} \hat{Y}_{1}\right)\left(t^{\alpha}\right)=\left(J_{0^{+}}^{2-\alpha} Z\right)(t), \quad 1<\alpha \leq 2,
$$

where $Z(t)=\left(\hat{Y}_{1}\left(t^{\alpha}\right)\right)^{\prime \prime}$. To compute $Z(t)$, we will apply twice the m.s. chain rule [46, Theorem 2.1.] with the following identification: $Y(t) \equiv \hat{Y}_{1}(t)$ and $g(t)=t^{\alpha}$. To legitimate this step, we need to assume that taking the second-order SPs $\hat{Y}_{1}(t)$ and $\hat{Y}_{1}^{\prime}(t)$ satisfy the following conditions D1-D4 (observe that $g(t)$ satisfies the hypotheses of [46, Theorem 2.1.]):

D1: $\hat{Y}_{1}(t)$ is m.s. differentiable at $v=t^{\alpha}$. Moreover,

$$
\hat{Y}_{1}^{\prime}\left(t^{\alpha}\right)=\sum_{m \geq 1} m X_{m} t^{\alpha(m-1)}
$$

D2: $\hat{Y}_{1}^{\prime}(t)$ is a m.s. differentiable at $v=t^{\alpha}$. Moreover,

$$
\hat{Y}_{1}^{\prime \prime}\left(t^{\alpha}\right)=\sum_{m \geq 2} m(m-1) X_{m} t^{\alpha(m-2)} .
$$

D3: $\frac{\mathrm{d} \hat{Y}_{1}(v)}{\mathrm{d} v}$ is m.s. continuous on $\left.v \in\right] 0,+\infty[$.

D4: $\frac{\mathrm{d}^{2} \hat{Y}_{1}(v)}{\mathrm{d}^{2} v}$ is m.s. continuous on $\left.v \in\right] 0,+\infty[$. 
In that case

$$
\begin{aligned}
Z(t)= & {\left[\left(\hat{Y}_{1}\left(t^{\alpha}\right)\right)^{\prime}\right]^{\prime}=\left[\left.\alpha t^{\alpha-1} \hat{Y}_{1}^{\prime}(v)\right|_{v=t^{\alpha}}\right]^{\prime} } \\
= & \left.\alpha(\alpha-1) t^{\alpha-2} \hat{Y}_{1}^{\prime}(v)\right|_{v=t^{\alpha}}+\left.\alpha t^{\alpha-1} \alpha t^{\alpha-1} \hat{Y}_{1}^{\prime \prime}(v)\right|_{v=t^{\alpha}} \\
= & \left.\alpha(\alpha-1) t^{\alpha-2} \hat{Y}_{1}^{\prime}(v)\right|_{v=t^{\alpha}}+\left.\alpha^{2} t^{2 \alpha-2} \hat{Y}_{1}^{\prime \prime}(v)\right|_{v=t^{\alpha}} \\
= & \alpha(\alpha-1) \sum_{m \geq 0}(m+1) X_{m+1} t^{\alpha(m+1)-2} \\
& +\alpha^{2} \sum_{m \geq 0}(m+2)(m+1) X_{m+2} t^{\alpha(m+2)-2}
\end{aligned}
$$

Observe that, we have applied Property (4.126) of [109, page 96] to compute the m.s. derivative of the product of the deterministic function $\alpha t^{\alpha-1}$ and the second-order SP $\hat{Y}_{1}^{\prime}\left(t^{\alpha}\right)$.

In order to legitimate the computation of the m.s. Caputo derivative $\left({ }^{C} D_{0^{+}}^{\alpha} Y_{1}\right)(t)$, we further assume that conditions D5 and D6 described below hold.

D5: The random generalized power series $\sum_{m \geq 0}(m+1) X_{m+1} t^{\alpha(m+1)-2}$ is m.s. uniformly convergent on $t>0$,

D6: The random generalized power series $\sum_{m \geq 0}(m+2)(m+1) X_{m+2} t^{\alpha(m+2)-2}$ is m.s. uniformly convergent on $t>0$,

Then,

$$
\begin{aligned}
& \left({ }^{C} D_{0^{+}}^{\alpha} Y_{1}\right)(t)=\left(J_{0^{+}}^{2-\alpha} Z\right)(t) \\
& =J_{0^{+}}^{2-\alpha}\left(\alpha(\alpha-1) \sum_{m \geq 0}(m+1) X_{m+1} t^{\alpha(m+1)-2}\right. \\
& \left.\quad+\alpha^{2} \sum_{m \geq 0}(m+2)(m+1) X_{m+2} t^{\alpha(m+2)-2}\right) \\
& =\alpha(\alpha-1) \sum_{m \geq 0}(m+1) X_{m+1} J_{0^{+}}^{2-\alpha}\left(t^{\alpha(m+1)-2}\right)
\end{aligned}
$$




$$
\begin{aligned}
& +\alpha^{2} \sum_{m \geq 0}(m+2)(m+1) X_{m+2} J_{0^{+}}^{2-\alpha}\left(t^{\alpha(m+2)-2}\right) \\
& =\alpha(\alpha-1) \sum_{m \geq 0}(m+1) X_{m+1}\left(\frac{1}{\Gamma(2-\alpha)} \int_{0}^{t}(t-u)^{1-\alpha} u^{\alpha(m+1)-2} \mathrm{~d} u\right) \\
& +\alpha^{2} \sum_{m \geq 0}(m+2)(m+1) X_{m+2}\left(\frac{1}{\Gamma(2-\alpha)} \int_{0}^{t}(t-u)^{1-\alpha} u^{\alpha(m+2)-2} \mathrm{~d} u\right) \\
& =\alpha(\alpha-1) \sum_{m \geq 0}(m+1) \frac{\Gamma(\alpha(m+1)-1)}{\Gamma(\alpha m+1)} X_{m+1} t^{\alpha m} \\
& +\alpha^{2} \sum_{m \geq 0}(m+2)(m+1) \frac{\Gamma(\alpha(m+2)-1)}{\Gamma(\alpha(m+1)+1)} X_{m+2} t^{\alpha(m+1)} \\
& =\alpha(\alpha-1) \Gamma(\alpha-1) X_{1}+\sum_{m \geq 1} \alpha(\alpha-1)(m+1) \frac{\Gamma(\alpha(m+1)-1)}{\Gamma(\alpha m+1)} X_{m+1} t^{\alpha m} \\
& +\alpha^{2} \sum_{m \geq 0}(m+2)(m+1) \frac{\Gamma(\alpha(m+2)-1)}{\Gamma(\alpha(m+1)+1)} X_{m+2} t^{\alpha(m+1)} \\
& =\Gamma(\alpha+1) X_{1}+\sum_{m \geq 0} \alpha(\alpha-1)(m+2) \frac{\Gamma(\alpha(m+2)-1)}{\Gamma(\alpha(m+1)+1)} X_{m+2} t^{\alpha(m+1)} \\
& +\sum_{m \geq 0} \alpha^{2}(m+2)(m+1) \frac{\Gamma(\alpha(m+2)-1)}{\Gamma(\alpha(m+1)+1)} X_{m+2} t^{\alpha(m+1)} \\
& =\Gamma(\alpha+1) X_{1}+\sum_{m \geq 0}(\alpha-1+\alpha(m+1)) \alpha(m+2) \frac{\Gamma(\alpha(m+2)-1)}{\Gamma(\alpha(m+1)+1)} X_{m+2} t^{\alpha(m+1)} \\
& =\Gamma(\alpha+1) X_{1}+\sum_{m \geq 0}(\alpha(m+2)-1) \alpha(m+2) \frac{\Gamma(\alpha(m+2)-1)}{\Gamma(\alpha(m+1)+1)} X_{m+2} t^{\alpha(m+1)} \\
& =\Gamma(\alpha+1) X_{1}+\sum_{m \geq 0} y \frac{\Gamma(\alpha(m+2)+1)}{\Gamma(\alpha(m+1)+1)} X_{m+2} t^{\alpha(m+1)} \\
& =\Gamma(\alpha+1) X_{1}+\sum_{m \geq 1} \frac{\Gamma(\alpha(m+1)+1)}{\Gamma(\alpha m+1)} X_{m+1} t^{\alpha m} \\
& =\sum_{m \geq 0} \frac{\Gamma(\alpha(m+1)+1)}{\Gamma(\alpha m+1)} X_{m+1} t^{\alpha m}
\end{aligned}
$$

where we have used the reproductive property of gamma function, $\Gamma(\gamma+1)=\gamma \Gamma(\gamma)$, $\gamma>0$ 
Now, we compute the random m.s. Caputo derivative of $Y_{2}(t)$. Note that by the definition of m.s. Caputo derivative (see Chapter 2) one gets

$$
\left({ }^{C} D_{0^{+}}^{\alpha} Y_{2}\right)(t)=\left(J_{0^{+}}^{2-\alpha} Y_{2}^{\prime \prime}\right)(t)=\left(J_{0^{+}}^{2-\alpha}\left(Y_{2}^{\prime}\right)^{\prime}\right)(t)=\left({ }^{C} D_{0^{+}}^{\alpha-1} Y_{2}^{\prime}\right)(t) \text {. }
$$

As $1<\alpha \leq 2$, and $Y_{2}^{\prime}(t)=\sum_{m \geq 0}(\alpha m+1) Y_{m} t^{\alpha m}$, we can recast $\left.\left.\hat{\alpha}=\alpha-1 \in\right] 0,1\right]$, $\hat{Y}_{m}=(\alpha m+1) Y_{m}$ and compute the random m.s. Caputo derivative of order $\hat{\alpha}$ of $\sum_{m \geq 0} \hat{Y}_{m} t^{\alpha m}$. Using the same argument shown in (2.25) one obtains

$$
\left({ }^{C} D_{0^{+}}^{\alpha} Y_{2}\right)(t)=\sum_{m \geq 0} Y_{m+1} \frac{\Gamma(\alpha(m+1)+2)}{\Gamma(\alpha m+2)} t^{\alpha m+1}
$$

Once we have obtained the m.s. Caputo derivative of both series given in (3.16), we need to compute their coefficients $X_{m}$ and $Y_{m}$. This can be done by substituting the expressions of the Caputo derivative of $Y_{1}(t)$ and $Y_{2}(t)$, given by (3.19) and (3.20), respectively, in random IVP $(3.1)$ taking into account that $\left({ }^{C} D_{0^{+}}^{\alpha} Y\right)(t)=$ $\left({ }^{C} D_{0^{+}}^{\alpha} Y_{1}\right)(t)+\left({ }^{C} D_{0^{+}}^{\alpha} Y_{2}\right)(t)$. This yields

$$
\begin{aligned}
\sum_{m \geq 0} & \frac{\Gamma(\alpha(m+1)+1)}{\Gamma(\alpha m+1)} X_{m+1} t^{\alpha m}-\lambda \sum_{m \geq 0} X_{m} t^{\alpha m} \\
& +\sum_{m \geq 0} \frac{\Gamma(\alpha(m+1)+2)}{\Gamma(\alpha m+2)} Y_{m+1} t^{\alpha m+1}-\lambda \sum_{m \geq 0} Y_{m} t^{\alpha m+1}=\gamma,
\end{aligned}
$$

thus

$$
\begin{aligned}
\Gamma(\alpha+1) X_{1}-\lambda X_{0} & +\sum_{m \geq 1}\left(\frac{\Gamma(\alpha(m+1)+1)}{\Gamma(\alpha m+1)} X_{m+1}-\lambda X_{m}\right) t^{\alpha m} \\
& +\sum_{m \geq 0}\left(\frac{\Gamma(\alpha(m+1)+2)}{\Gamma(\alpha m+2)} Y_{m+1}-\lambda Y_{m}\right) t^{\alpha m+1}=\gamma
\end{aligned}
$$

If the following recurrences for coefficients $X_{m}$

$$
X_{1}=\frac{\lambda X_{0}+\gamma}{\Gamma(\alpha+1)}, \quad X_{m+1}=\frac{\lambda \Gamma(\alpha m+1)}{\Gamma(\alpha(m+1)+1)} X_{m}, \quad m \geq 1
$$


and $Y_{m}$

$$
Y_{m+1}=\frac{\lambda \Gamma(\alpha m+2)}{\Gamma(\alpha(m+1)+2)} Y_{m}, \quad m \geq 0
$$

are satisfied, then it is guaranteed that the relationship (3.22) hold. Taking into account the initial conditions $Y(0)=X_{0}=\beta_{0}$ and $Y^{\prime}(0)=Y_{0}=\beta_{1}$, and using recurrences (3.23) and (3.24) one gets

$$
X_{m}=\frac{\lambda^{m} \beta_{0}+\lambda^{m-1} \gamma}{\Gamma(\alpha m+1)}, \quad Y_{m}=\frac{\lambda^{m} \beta_{1}}{\Gamma(\alpha m+2)}, \quad m \geq 1 .
$$

Therefore, a candidate solution SP to the random IVP (3.1) with $1<\alpha \leq 2$ is given by

$$
Y(t)=\sum_{m \geq 0} X_{m, 1} t^{\alpha m}+\sum_{m \geq 1} X_{m, 2} t^{\alpha m}+\sum_{m \geq 0} Y_{m} t^{\alpha m+1}
$$

where

$$
X_{m, 1}=\frac{\lambda^{m} \beta_{0}}{\Gamma(\alpha m+1)}, \quad X_{m, 2}=\frac{\lambda^{m-1} \gamma}{\Gamma(\alpha m+1)}, \quad Y_{m}=\frac{\lambda^{m} \beta_{1}}{\Gamma(\alpha m+2)} .
$$

Observe that for convenience, the general term of series $X_{m} t^{\alpha m}$ has been split in two pieces. So far, we have constructed a formal solution SP to random IVP (3.1) and now, assuming that input RVs satisfy hypotheses $\hat{\mathbf{H}} \mathbf{1}$ and $\hat{\mathbf{H}} \mathbf{2}$, we need to check that conditions D1-D6 hold. As this can be done by taking the same steps shown in detail in [24], they will be skipped here. The analysis of m.s. convergence of (3.25)-(3.26) can be carried out as shown in Case I since the involved series are identical and/or very similar, hence we omit this discussion.

To compute approximations for the mean of the solution SP $Y(t)$, we first consider the truncation of order, say $M$, of the infinite series (3.25)-(3.26), i.e.,

$$
Y_{M}(t)=\sum_{m=0}^{M} X_{m, 1} t^{\alpha m}+\sum_{m=1}^{M} X_{m, 2} t^{\alpha m}+\sum_{m=0}^{M} Y_{m} t^{\alpha m+1},
$$


and then, we take the expectation operator. Using independence of $\beta_{0}, \beta_{1}, \gamma$ and $\lambda$ (see $\hat{\mathbf{H}} \mathbf{1})$, one obtains

$$
\begin{aligned}
\mathbb{E}\left[Y_{M}(t)\right] & =\mathbb{E}\left[\beta_{0}\right] \sum_{m=0}^{M} \frac{\mathbb{E}\left[\lambda^{m}\right]}{\Gamma(\alpha m+1)} t^{\alpha m}+\mathbb{E}[\gamma] \sum_{m=1}^{M} \frac{\mathbb{E}\left[\lambda^{m-1}\right]}{\Gamma(\alpha m+1)} t^{\alpha m} \\
& +\mathbb{E}\left[\beta_{1}\right] \sum_{m=0}^{M} \frac{\mathbb{E}\left[\lambda^{m}\right]}{\Gamma(\alpha m+2)} t^{\alpha m+1} .
\end{aligned}
$$

Instead of providing approximations for the variance (or standard deviation) function of $Y(t)$, we will give more general approximations. Indeed, our first step will obtain approximations of the cross-covariance function of $Y(t), \mathbb{C}_{Y_{M}, Y_{N}}(t, s)$, by considering two different truncations $Y_{M}(t)$ and $Y_{N}(s)$ at the points $t$ and $s$, respectively,

$$
\begin{aligned}
\mathbb{C}_{Y_{M}, Y_{N}}(t, s)= & \sum_{m=0}^{M} \sum_{n=0}^{N} \mathbb{C o v}\left[X_{m, 1}, X_{n, 1}\right] t^{\alpha m} s^{\alpha n} \\
& +\sum_{m=0}^{M} \sum_{n=1}^{N} \operatorname{Cov}\left[X_{m, 1}, X_{n, 2}\right] t^{\alpha m} s^{\alpha n} \\
& +\sum_{m=0}^{M} \sum_{n=0}^{N} \mathbb{C o v}\left[X_{m, 1}, Y_{n}\right] t^{\alpha m} s^{\alpha n+1} \\
& +\sum_{m=1}^{M} \sum_{n=0}^{N} \mathbb{C o v}\left[X_{m, 2}, X_{n, 1}\right] t^{\alpha m} s^{\alpha n} \\
& +\sum_{m=1}^{M} \sum_{n=1}^{N} \mathbb{C o v}\left[X_{m, 2}, X_{n, 2}\right] t^{\alpha m} s^{\alpha n} \\
& +\sum_{m=1}^{M} \sum_{n=0}^{N} \mathbb{C o v}\left[X_{m, 2}, Y_{n}\right] t^{\alpha m} s^{\alpha n+1} \\
& +\sum_{m=0}^{M} \sum_{n=0}^{N} \mathbb{C o v}\left[Y_{m}, Y_{n}\right] t^{\alpha m+1} s^{\alpha n+1} \\
& +\sum_{m=0}^{M} \sum_{n=1}^{N} \mathbb{C o v}\left[Y_{m}, X_{n, 2}\right] t^{\alpha m+1} s^{\alpha n}
\end{aligned}
$$




$$
+\sum_{m=0}^{M} \sum_{n=1}^{N} \operatorname{Cov}\left[Y_{m}, Y_{n}\right] t^{\alpha m+1} s^{\alpha n+1}
$$

where $\mathbb{C o v}[\cdot, \cdot]$ denotes the covariance operator. Applying hypothesis $\hat{\mathbf{H}} \mathbf{1}$, each covariance can be expressed in terms of data as follows

$$
\begin{aligned}
\operatorname{Cov}\left[X_{m, 1}, X_{n, 1}\right] & =\frac{\mathbb{E}\left[\lambda^{m+n}\right] \mathbb{E}\left[\left(\beta_{0}\right)^{2}\right]-\mathbb{E}\left[\lambda^{m}\right] \mathbb{E}\left[\lambda^{n}\right]\left(\mathbb{E}\left[\beta_{0}\right]\right)^{2}}{\Gamma(\alpha m+1) \Gamma(\alpha n+1)}, \\
\operatorname{Cov}\left[X_{m, 1}, X_{n, 2}\right] & =\frac{\left(\mathbb{E}\left[\lambda^{m+n-1}\right]-\mathbb{E}\left[\lambda^{m}\right] \mathbb{E}\left[\lambda^{n-1}\right]\right) \mathbb{E}\left[\beta_{0}\right] \mathbb{E}[\gamma]}{\Gamma(\alpha m+1) \Gamma(\alpha n+1)}, \\
\operatorname{Cov}\left[X_{m, 1}, Y_{n}\right] & =\frac{\left(\mathbb{E}\left[\lambda^{m+n}\right]-\mathbb{E}\left[\lambda^{m}\right] \mathbb{E}\left[\lambda^{n}\right]\right) \mathbb{E}\left[\beta_{0}\right] \mathbb{E}\left[\beta_{1}\right]}{\Gamma(\alpha m+1) \Gamma(\alpha n+2)}, \\
\operatorname{Cov}\left[X_{m, 2}, X_{n, 1}\right] & =\frac{\left(\mathbb{E}\left[\lambda^{m+n-1}\right]-\mathbb{E}\left[\lambda^{m-1}\right] \mathbb{E}\left[\lambda^{n}\right]\right) \mathbb{E}\left[\beta_{0}\right] \mathbb{E}[\gamma]}{\Gamma(\alpha m+1) \Gamma(\alpha n+1)} \\
\operatorname{Cov}\left[X_{m, 2}, X_{n, 2}\right] & =\frac{\mathbb{E}\left[\lambda^{m+n-2}\right] \mathbb{E}\left[\gamma^{2}\right]-\mathbb{E}\left[\lambda^{m-1}\right] \mathbb{E}\left[\lambda^{n-1}\right](\mathbb{E}[\gamma])^{2}}{\Gamma(\alpha m+1) \Gamma(\alpha n+1)}, \\
\operatorname{Cov}\left[X_{m, 2}, Y_{n}\right] & =\frac{\left(\mathbb{E}\left[\lambda^{m+n-1}\right]-\mathbb{E}\left[\lambda^{m-1}\right] \mathbb{E}\left[\lambda^{n}\right]\right) \mathbb{E}[\gamma] \mathbb{E}\left[\beta_{1}\right]}{\Gamma(\alpha m+1) \Gamma(\alpha n+2)}, \\
\operatorname{Cov}\left[Y_{m}, X_{n, 1}\right] & =\frac{\left(\mathbb{E}\left[\lambda^{m+n}\right]-\mathbb{E}\left[\lambda^{m}\right] \mathbb{E}\left[\lambda^{n}\right]\right) \mathbb{E}\left[\beta_{0}\right] \mathbb{E}\left[\beta_{1}\right]}{\Gamma(\alpha m+2) \Gamma(\alpha n+1)}, \\
\operatorname{Cov}\left[Y_{m}, X_{n, 2}\right] & =\frac{\left(\mathbb{E}\left[\lambda^{m+n-1}\right]-\mathbb{E}\left[\lambda^{m}\right] \mathbb{E}\left[\lambda^{n-1}\right]\right) \mathbb{E}[\gamma] \mathbb{E}\left[\beta_{1}\right]}{\Gamma(\alpha m+2) \Gamma(\alpha n+1)}, \\
\mathbb{C o v}\left[Y_{m}, Y_{n}\right] & =\frac{\mathbb{E}\left[\lambda^{m+n}\right] \mathbb{E}\left[\beta_{1}^{2}\right]-\mathbb{E}\left[\lambda^{m}\right] \mathbb{E}\left[\lambda^{n}\right]\left(\mathbb{E}\left[\beta_{1}\right]\right)^{2}}{\Gamma(\alpha m+2) \Gamma(\alpha n+1)} .
\end{aligned}
$$

If we take $M=N$ in (3.29), we then obtain the covariance function, $\mathbb{C}_{Y_{M}}(t, s)$, of the approximation $Y_{M}(t)$, while its variance function is derived taking $t=s$ in the covariance function, i.e.,

$$
\mathbb{C}_{Y_{M}}(t, s)=\mathbb{C}_{Y_{M}, Y_{M}}(t, s), \quad \mathbb{V}\left[Y_{M}(t)\right]=\mathbb{C}_{Y_{M}}(t, t) .
$$

Summarizing the following result has been established 
Theorem 3.2 Let us consider the random fractional IVP (3.1) with $1<\alpha \leq 2$ and assume that the inputs data $\beta_{0}, \beta_{1}, \gamma$ and $\lambda$ are RVs satisfying hypotheses $\hat{\mathbf{H}} \mathbf{1}$ and $\hat{\mathbf{H} 2}$. If $p \geq 0$ and $\alpha \in] 1,2]$ are so that $p<\alpha$, then the random generalized power series $Y(t)$ given by (3.25)-(3.26) is a m.s. solution to the IVP (3.1) for all $t \geq 0$. While, if $p=\alpha$, then $Y(t)$ is a m.s. solution to the IVP (3.1) over the domain $t: 0 \leq t<\alpha / \mathcal{H}^{\frac{1}{\alpha}}$. Furthermore, the approximations of the mean and the variance (or standard deviation) given by (3.28) and (3.29)-(3.31), respectively, will also converge at least over the domains previously specified for the m.s. convergence.

Similar comments to the ones contained in Remark 3.2 can now be made with respect to the intervals of convergence to the mean and the variance determined in Theorem 3.2.

\subsection{Numerical examples}

This section is devoted to illustrate, through a variety of examples, the results established in Theorems 3.1 and 3.2. Particularly we investigate, through examples, if the domain of convergence of the mean of the solution SP to the random fractional IVP (3.1) can be enlarger with respect the one inferred from the m.s. convergence. This issue will be discussed through the approximations for statistical moments given in Section 3.3. The examples have been devised to take into consideration both bounded and unbounded RVs for the diffusion coefficient $\lambda$. In the examples, the accuracy of the approximations of the mean and standard deviation will be measured using the following relative errors ( $\mathrm{RE}$ ) between consecutive approximations of order $M$ and $M+1$, using different values of $M$ and different time instants $t$,

$$
\begin{gathered}
\operatorname{RE}(\text { Mean })(t ; M)=\left|\frac{\mathbb{E}\left[Y_{M+1}(t)\right]-\mathbb{E}\left[Y_{M}(t)\right]}{\mathbb{E}\left[Y_{M}(t)\right]}\right|, \\
\operatorname{RE}(\operatorname{Sd})(t ; M)=\left|\frac{\sqrt{\mathbb{V}\left[Y_{M+1}(t)\right]}-\sqrt{\mathbb{V}\left[Y_{M}(t)\right]}}{\sqrt{\mathbb{V}\left[Y_{M}(t)\right]}}\right| .
\end{gathered}
$$

Here, $\mathbb{E}\left[Y_{M}(t)\right]$ and $\mathbb{V}\left[Y_{M}(t)\right]$ are given by expressions (2.40) and (2.42), in Case I, and by (3.29)-(3.31), in Case II, respectively. 
Example 3.5 This example illustrates Case I, corresponding to $\alpha \in] 0,1]$, when diffusion coefficient $\lambda$ is a bounded $R V$. Let us consider the random fractional IVP (3.1), where

- $\beta_{0}$ is an Exponential RV of mean $1 / 5$ and variance $1 / 25$, i.e., $\beta_{0} \sim \operatorname{Exp}(5)$.

- $\gamma$ is a Gaussian $R V$ with zero mean and unit standard deviation, $\gamma \sim N(0 ; 1)$.

- $\lambda$ is a Beta $R V$ of mean $2 / 5$ and variance $1 / 25, \lambda \sim B e(2 ; 3)$.

We will also assume that $\beta_{0}, \gamma$ and $\lambda$ are independent $R V$ s. Since $\lambda$ is a bounded $R V$ (it lies on the interval ]0,1[), by Example 3.1 we know that $\lambda$ satisfies hypothesis H2. Also, clearly all these input data are second-order RVs because they have finite variance. As a consequence, hypothesis $\hat{\mathbf{H}} \mathbf{1}$ also holds and Theorem3.1 can be applied. Observe that the parameter $p$ associated to $\lambda$ is $p=0$ (see Example 3.1). According to Theorem 3.1 the solution $Y(t)$, given by (3.15), is m.s. convergent for all $t \geq 0$. Therefore, the expectation and the variance (or equivalently, the standard deviation) of $Y(t)$, which are given by (2.40) and (2.42), respectively, will also converge for all $t \geq 0$, independently of the order $\alpha \in] 0,1]$ of the fractional derivative. This conclusion is illustrated in Figure $3.1(\alpha=0.3)$ and in Figure 3.2 $(\alpha=0.7)$ over the time intervals $0 \leq t \leq 5$ and $0 \leq t \leq 8$, respectively, using different orders of truncation $M$. Observe that both values of $\alpha \in] 0,1]$, hence they correspond to Case I. From both graphical representations we observe that the approximations of the mean and the standard deviation converge over the whole interval. Moreover, these approximations improve as $M$ increases.

In Tables 3.2 and 3.3 we have collected the figures of relative errors of the approximations of the mean and standard deviation defined in (3.32) and (3.33), respectively. Both tables correspond to $\alpha=0.3$. We observe that for $t$ fixed both errors decrease as $M$ increases, while for a fixed truncation order $M$ these errors increase as $t$ departs from the origin $t=0$. An analogous analysis corresponding to $\alpha=0.7$ is shown in Tables 3.4 and 3.5. In these tables, the numerical results are only shown in several points placed near the right-end of the interval $0 \leq t \leq 8$ in order to better observe how evolves that error and to account for its maximum value. Specifically, we have listed the relative errors for $t=4,5,6,7,8$, just to be clearer. 

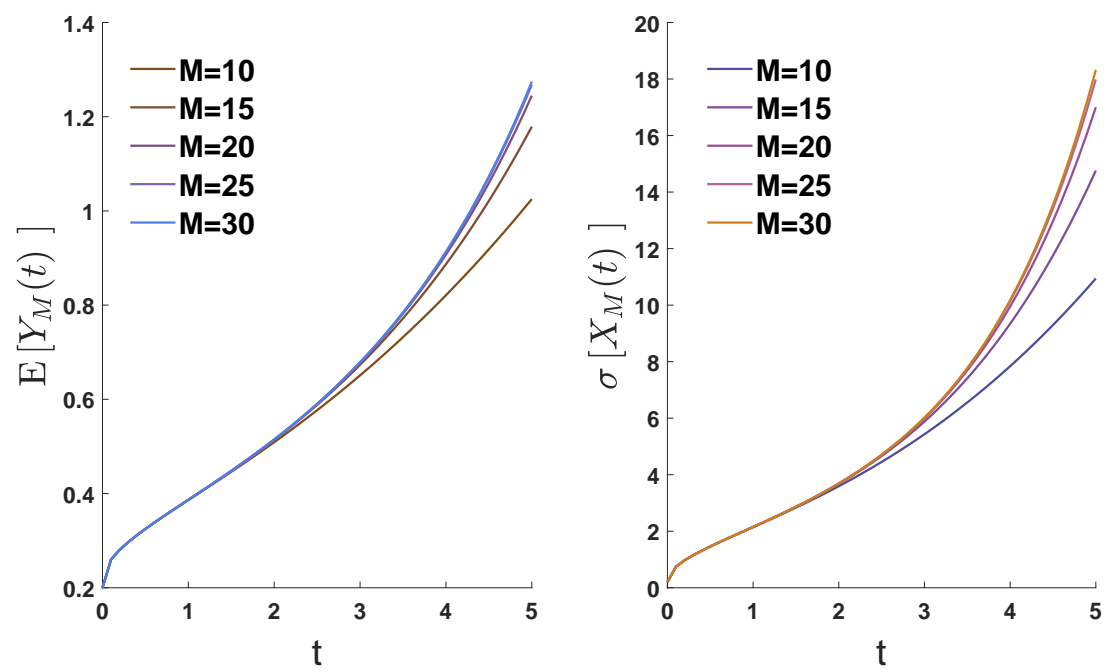

Figure 3.1: Approximations of the mean (left) and the standard deviation (right) of the solution SP to the random fractional IVP (3.1) with $\alpha=0.3$ (Case I) taking different orders of truncation $M$ over the time interval $[0,5]$ in the context of Example 3.5.

\begin{tabular}{|c|c|c|c|c|c|}
\hline RE (Mean) $(t ; M)$ & $t=1$ & $t=2$ & $t=3$ & $t=4$ & $t=5$ \\
\hline$M=15$ & $4.079521 \mathrm{e}-05$ & $6.947839 \mathrm{e}-04$ & $3.297477 \mathrm{e}-03$ & $9.189631 \mathrm{e}-03$ & $1.905735 \mathrm{e}-02$ \\
\hline$M=20$ & $1.419749 \mathrm{e}-06$ & $6.827955 \mathrm{e}-05$ & $5.907660 \mathrm{e}-04$ & $2.484655 \mathrm{e}-03$ & $6.938988 \mathrm{e}-03$ \\
\hline$M=25$ & $4.034508 \mathrm{e}-08$ & $5.487145 \mathrm{e}-06$ & $8.709276 \mathrm{e}-05$ & $5.606024 \mathrm{e}-04$ & $2.154161 \mathrm{e}-03$ \\
\hline$M=40$ & $3.261758 \mathrm{e}-13$ & $1.003650 \mathrm{e}-09$ & $9.874694 \mathrm{e}-08$ & $2.315692 \mathrm{e}-06$ & $2.412006 \mathrm{e}-05$ \\
\hline$M=50$ & $0.000000 \mathrm{e}-32$ & $1.570471 \mathrm{e}-12$ & $5.214614 \mathrm{e}-10$ & $2.898630 \mathrm{e}-08$ & $5.896433 \mathrm{e}-07$ \\
\hline
\end{tabular}

Table 3.2: Numerical values of the relative error (3.32) corresponding to the approximations of the mean of the solution SP to the random IVP (3.1) with $\alpha=0.3$ (Case I) at different values of $t$ and $M$ in the context of Example 3.5.

\begin{tabular}{|c|c|c|c|c|c|}
\hline $\mathrm{RE}(\mathrm{Sd})(t ; M)$ & $t=1$ & $t=2$ & $t=3$ & $t=4$ & $t=5$ \\
\hline$M=15$ & $9.638948 \mathrm{e}-05$ & $1.757966 \mathrm{e}-03$ & $8.611555 \mathrm{e}-03$ & $2.338490 \mathrm{e}-02$ & $4.545151 \mathrm{e}-02$ \\
\hline$M=20$ & $3.514650 \mathrm{e}-06$ & $1.914465 \mathrm{e}-04$ & $1.802353 \mathrm{e}-03$ & $7.588380 \mathrm{e}-03$ & $1.970048 \mathrm{e}-02$ \\
\hline$M=25$ & $1.032923 \mathrm{e}-07$ & $1.650448 \mathrm{e}-05$ & $2.962502 \mathrm{e}-04$ & $1.971703 \mathrm{e}-03$ & $7.119996 \mathrm{e}-03$ \\
\hline$M=40$ & $8.883801 \mathrm{e}-13$ & $3.424193 \mathrm{e}-09$ & $4.063098 \mathrm{e}-07$ & $1.050714 \mathrm{e}-05$ & $1.086227 \mathrm{e}-04$ \\
\hline$M=50$ & $0.000000 \mathrm{e}-32$ & $5.631090 \mathrm{e}-12$ & $2.312461 \mathrm{e}-09$ & $1.452893 \mathrm{e}-07$ & $3.004525 \mathrm{e}-06$ \\
\hline
\end{tabular}

Table 3.3: Numerical values of the relative error (3.33) corresponding to the standard deviation of the solution SP to the random IVP (3.1) with $\alpha=0.3$ (Case I) at different values of $t$ and $M$ in the context of Example 3.5. 

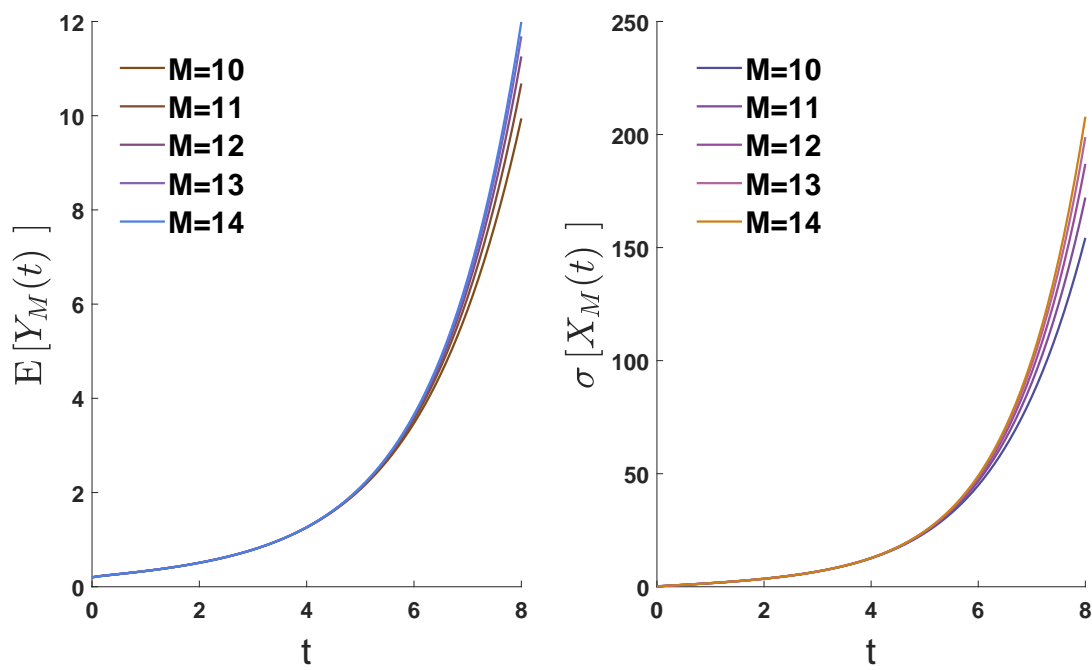

Figure 3.2: Approximations of the mean (left) and the standard deviation (right) of the solution SP to the random fractional IVP (3.1) with $\alpha=0.7$ (Case I) taking different orders of truncation $M$ over the time interval $[0,8]$ in the context of Example 3.5.

\begin{tabular}{|c|c|c|c|c|c|}
\hline RE(Mean) $(\mathrm{t} ; \mathrm{M})$ & $t=4$ & $t=5$ & $t=6$ & $t=7$ & $t=8$ \\
\hline$M=11$ & $2.834329 \mathrm{e}-03$ & $9.606890 \mathrm{e}-03$ & $2.328737 \mathrm{e}-02$ & $4.504716 \mathrm{e}-02$ & $7.455311 \mathrm{e}-02$ \\
\hline$M=12$ & $1.349031 \mathrm{e}-03$ & $5.309681 \mathrm{e}-03$ & $1.442739 \mathrm{e}-02$ & $3.044101 \mathrm{e}-02$ & $5.379721 \mathrm{e}-02$ \\
\hline$M=13$ & $6.168547 \mathrm{e}-04$ & $2.827171 \mathrm{e}-03$ & $8.649223 \mathrm{e}-03$ & $2.001286 \mathrm{e}-02$ & $3.797259 \mathrm{e}-02$ \\
\hline$M=14$ & $2.714154 \mathrm{e}-04$ & $1.451050 \mathrm{e}-03$ & $5.014414 \mathrm{e}-03$ & $1.278055 \mathrm{e}-02$ & $2.616524 \mathrm{e}-02$ \\
\hline$M=20$ & $9.626787 \mathrm{e}-07$ & $1.310833 \mathrm{e}-05$ & $9.664635 \mathrm{e}-05$ & $4.614136 \mathrm{e}-04$ & $1.592402 \mathrm{e}-03$ \\
\hline
\end{tabular}

Table 3.4: Numerical values of the relative error (3.32) corresponding to the mean of the solution SP to the random IVP (3.1) with $\alpha=0.7$ (Case I) at different values of $t$ and $M$ in the context of Example 3.5.

\begin{tabular}{|c|c|c|c|c|c|}
\hline $\mathrm{RE}(\mathrm{Sd})(t ; M)$ & $t=4$ & $t=5$ & $t=6$ & $t=7$ & $t=8$ \\
\hline$M=11$ & $4.974931 \mathrm{e}-03$ & $1.682744 \mathrm{e}-02$ & $3.957739 \mathrm{e}-02$ & $7.319679 \mathrm{e}-02$ & $1.155156 \mathrm{e}-01$ \\
\hline$M=12$ & $2.444194 \mathrm{e}-03$ & $9.685880 \mathrm{e}-03$ & $2.561774 \mathrm{e}-02$ & $5.156699 \mathrm{e}-02$ & $8.645137 \mathrm{e}-02$ \\
\hline$M=13$ & $1.149708 \mathrm{e}-03$ & $5.353075 \mathrm{e}-03$ & $1.601557 \mathrm{e}-02$ & $3.533860 \mathrm{e}-02$ & $6.335321 \mathrm{e}-02$ \\
\hline$M=14$ & $5.188710 \mathrm{e}-04$ & $2.842382 \mathrm{e}-03$ & $9.660322 \mathrm{e}-03$ & $2.350868 \mathrm{e}-02$ & $4.535487 \mathrm{e}-02$ \\
\hline$M=20$ & $2.058083 \mathrm{e}-06$ & $2.984451 \mathrm{e}-05$ & $2.239663 \mathrm{e}-04$ & $1.045773 \mathrm{e}-03$ & $3.432793 \mathrm{e}-03$ \\
\hline
\end{tabular}

Table 3.5: Numerical values of the relative error (3.33) corresponding to the standard deviation of the solution SP to the random IVP (3.1) with $\alpha=0.7$ (Case I) at different values of $t$ and $M$ in the context of Example 3.5. 
Example 3.6 This example illustrates Case I, corresponding to $\alpha \in] 0,1]$, when diffusion coefficient $\lambda$ is an unbounded $R V$. Let us consider the random fractional $\operatorname{IVP}(3.1)$, where

- $\beta_{0}$ is a Gamma $R V$ of mean $1 / 5$ and variance $1 / 25$, i.e. $\beta_{0} \sim G a(1 ; 1 / 5)$.

- $\gamma$ is a Beta $R V$ of mean $1 / 4$ and variance $1 / 50$, $\lambda \sim \operatorname{Be}(67 / 32 ; 201 / 32)$.

- $\lambda$ is a Gaussian $R V$ with zero mean and standard deviation $1 / 10, \gamma$ $N\left(0 ;(1 / 10)^{2}\right)$.

We will also assume that $\beta_{0}, \gamma$ and $\lambda$ are independent $R V$ s. Observe that in this example $\lambda$ is an unbounded $R V$ and, according to Example 3.2, it satisfies hypothesis $\hat{\mathbf{H} 2}$ with $p=1 / 2, \mathcal{H}=\sqrt{2} / 10$ and $\eta=1 / 10$. Hypothesis $\mathbf{H 1}$ also fulfils because all input data are assumed to be independent and they have finite variance. Therefore, according to Theorem 3.1, the random generalized power series solution $Y(t)$, given by (3.15), is m.s. convergent in a domain that depends on the relationship between $p=1 / 2$ and $\alpha$. In this example, we will only consider the Case $I$, thus $\alpha \in] 0,1]$. Specifically, for $\alpha \in] 1 / 2,1]$, that is, when $p<\alpha, Y(t)$ is m.s. convergent for all $t \geq 0$, and, as a consequence, the approximations (2.40) and (2.42) for the mean and the variance (or standard deviation), respectively, will also converge for all $t \geq 0$. While if $\alpha=p=1 / 2, Y(t)$ is m.s. convergent over the domain $0 \leq t<25$. Notice that the right-end value of this interval corresponds to $\alpha / \mathcal{H}^{1 / \alpha}$. In this case, it is guaranteed that the approximations of both the mean and the variance will converge, at least, in this same interval $0 \leq t<25$, although this domain could be larger. This question will be further discussed later.

Firstly, we illustrate the former finding in Figure 3.3 where we have taken $\alpha=0.6$ (Case I) as the fractional order of the derivative. In this graphical representation, we have plotted approximations of the mean and the standard deviation over the time interval $0 \leq t \leq 30$ using different orders of truncation $M$. In Tables 3.6 and 3.7 the numerical values of relative errors, defined in (3.32) and (3.33), at some selected values are shown. From these figures we can conclude the proposed method gives good and reliable approximations. 


\begin{tabular}{|c|c|c|c|c|c|}
\hline $\mathrm{RE}(\operatorname{mean})(t ; M)$ & $t=6$ & $t=7$ & $t=8$ & $t=9$ & $t=10$ \\
\hline$M=15$ & $1.787302 \mathrm{e}-06$ & $6.526650 \mathrm{e}-06$ & $1.986069 \mathrm{e}-05$ & $5.253359 \mathrm{e}-05$ & $1.242889 \mathrm{e}-04$ \\
\hline$M=20$ & $3.228105 \mathrm{e}-08$ & $1.871873 \mathrm{e}-07$ & $8.502444 \mathrm{e}-07$ & $3.201927 \mathrm{e}-06$ & $1.038967 \mathrm{e}-05$ \\
\hline$M=25$ & $3.122633 \mathrm{e}-10$ & $2.875344 \mathrm{e}-09$ & $1.949542 \mathrm{e}-08$ & $1.045336 \mathrm{e}-07$ & $4.652778 \mathrm{e}-07$ \\
\hline$M=40$ & $8.693692 \mathrm{e}-16$ & $3.250538 \mathrm{e}-14$ & $7.327634 \mathrm{e}-13$ & $1.134049 \mathrm{e}-11$ & $1.302890 \mathrm{e}-10$ \\
\hline$M=50$ & $0.000000 \mathrm{e}-32$ & $0.000000 \mathrm{e}-32$ & $3.626644 \mathrm{e}-16$ & $1.478768 \mathrm{e}-14$ & $3.211297 \mathrm{e}-13$ \\
\hline
\end{tabular}

Table 3.6: Numerical values of the relative error (3.32) corresponding to the mean of the SP to the random IVP (3.1) with $\alpha=0.6$ (Case I) in different values of $t$ and $M$ in the context of Example 3.6.

\begin{tabular}{|c|c|c|c|c|c|}
\hline $\mathrm{RE}(\mathrm{Sd})(t ; M)$ & $t=6$ & $t=7$ & $t=8$ & $t=9$ & $t=10$ \\
\hline$M=15$ & $5.637725 \mathrm{e}-05$ & $2.633044 \mathrm{e}-04$ & $9.870608 \mathrm{e}-04$ & $3.057817 \mathrm{e}-03$ & $7.947836 \mathrm{e}-03$ \\
\hline$M=20$ & $1.702671 \mathrm{e}-06$ & $1.447403 \mathrm{e}-05$ & $9.378429 \mathrm{e}-05$ & $4.811486 \mathrm{e}-04$ & $1.975614 \mathrm{e}-03$ \\
\hline$M=25$ & $4.642409 \mathrm{e}-08$ & $7.069181 \mathrm{e}-07$ & $7.818432 \mathrm{e}-06$ & $6.610486 \mathrm{e}-05$ & $4.333418 \mathrm{e}-04$ \\
\hline$M=40$ & $5.354437 \mathrm{e}-13$ & $4.653621 \mathrm{e}-11$ & $2.484909 \mathrm{e}-09$ & $9.011051 \mathrm{e}-08$ & $2.327453 \mathrm{e}-06$ \\
\hline$M=50$ & $0.000000 \mathrm{e}-32$ & $5.305710 \mathrm{e}-14$ & $8.013567 \mathrm{e}-12$ & $7.473559 \mathrm{e}-10$ & $4.663530 \mathrm{e}-08$ \\
\hline
\end{tabular}

Table 3.7: Numerical values of the relative error (3.33) corresponding to the standard deviation of the SP to the random IVP (3.1) with $\alpha=0.6$ (Case I) in different values of $t$ and $M$ in the context of Example 3.6.
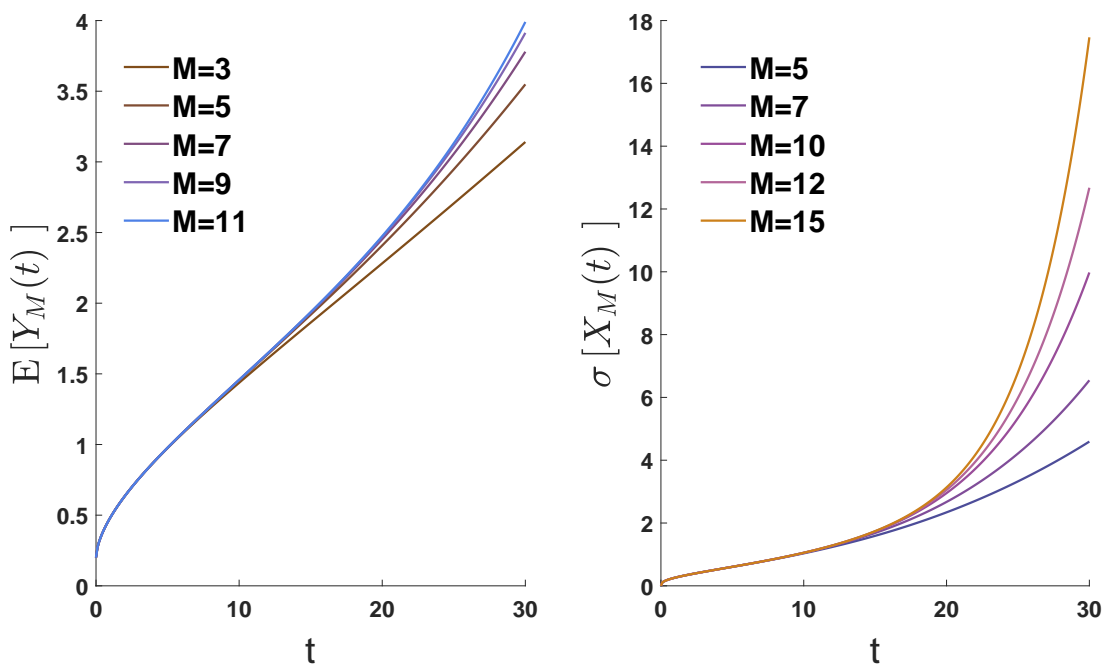

Figure 3.3: Approximations of the mean (left) and the standard deviation (right) of the solution SP to the random fractional IVP (3.1) with $\alpha=0.6$ (Case I) taking different orders of truncation $M$ over the time interval $[0,30]$ in the context of Example 3.6. 
Secondly, we show and analyze the results obtained in the case that $p=\alpha=1 / 2$. On the left-side of Figure 3.4 we have plotted the approximations of the mean over the time interval $0 \leq t \leq 60$ for different values of $M$, while the approximations of the standard deviation have been represented over a shorter interval, namely $0 \leq t \leq 30$ (see right-side of Figure 3.4). This is an important point in our analysis regarding the case where m.s. convergence takes place in a bounded interval, i.e. when $p=\alpha=1 / 2$ (see Theorem 3.1). Observe that, according to this theorem, the approximations of the mean and the variance (standard deviation) of the solution have the same domain of convergence. This domain is inferred from the one where m.s. convergence takes place. If we revise the proof of Theorem 3.1, we can realize that it provides a sufficient condition for m.s. convergence which relies upon the construction of a convergent majorizing series. Although the result is fair general, it does not guarantee the domain of m.s. convergence of the solution $S P Y(t)$ (and hence of the approximations of its mean and variance), could be larger. In order to illustrate this issue, now we will show, with input data of our example, that the approximation of the mean converges over the larger time interval $0 \leq t<50$, while the approximation of the variance converges over the time interval $0 \leq t<25$. Therefore, this is in fully agreement with the numerical results exhibited in Figure 3.4. Additionally, we have computed and plotted the relatives errors (3.32) and (3.33) of approximations for the mean and the standard deviation. The graphical representation of these errors are shown in Figure 3.5 using different orders of truncation $M=50,60,70,80,90$. For the sake of clarity in this plot we have included a zoom of around the critical points $t=50$ (for the mean) and $t=25$ (for the standard deviation). From this plot, we clearly observe that divergence of approximations of the mean and the standard deviation occur after the critical points $t=50$ and $t=25$, respectively.

As expected, the interval of convergence of the standard deviation matches the one inferred from the analysis of the m.s. convergence. While the interval of convergence to the mean is larger. Now, we justify this latter numerical result using analytic arguments. This fact is intuitive since m.s. convergence involves information of the second order moment (which is related to the variance/standard deviation) rather than first order moment (related to the mean). To completely support this intuition, we now prove that the interval of convergence of the deterministic series 

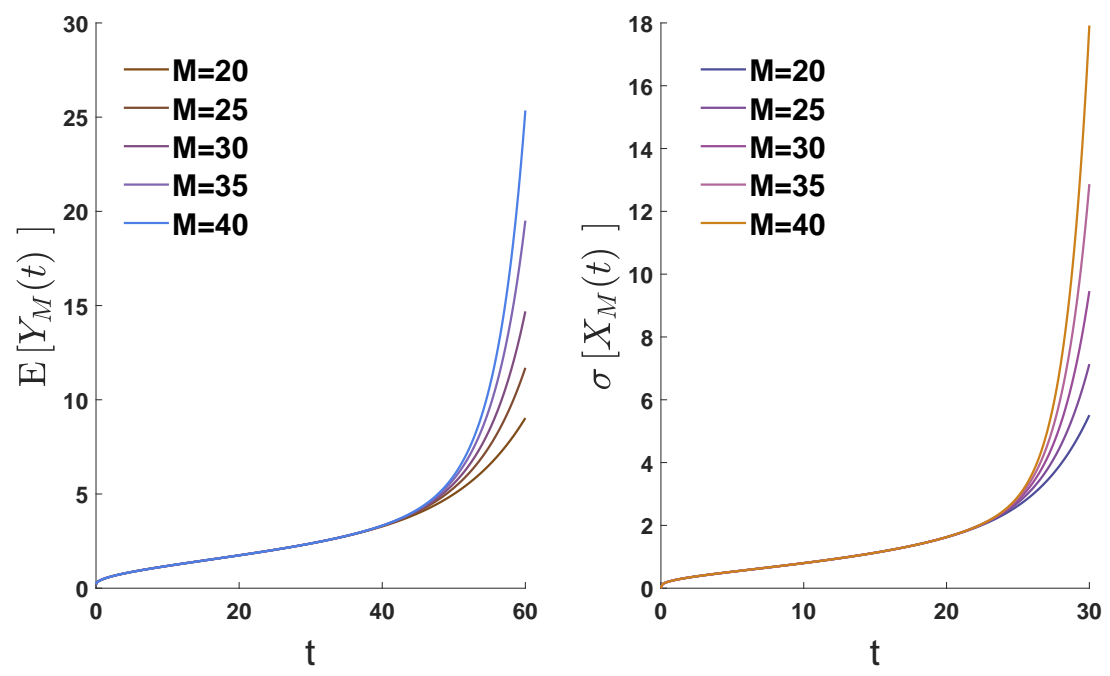

Figure 3.4: Approximations of the mean (left) and the standard deviation (right) of the solution SP to the random fractional IVP (3.1) with $\alpha=0.5$ (Case I) using different orders of truncation $M$ over the time intervals $[0,60]$ and $[0,30]$, respectively in the context of Example 3.6 .
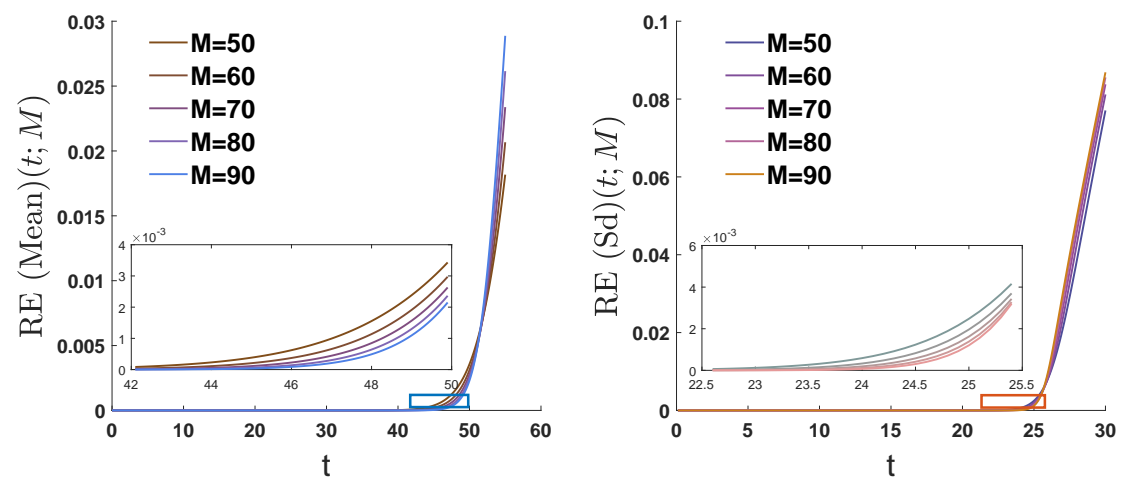

Figure 3.5: Relative errors, given in (3.32) and (3.33), of the approximations of the mean (left) and the standard deviation (right) of the solution SP to the random fractional IVP (3.1) with $\alpha=0.5$ (Case I) using different orders of truncation $M$ over the time intervals $[0,60]$ and $[0,30]$, respectively, in the context of Example 3.6. For the sake of clarity, in both plots, we present a zoom around of the end-points $t=50$ and $t=25$ of the convergence regions for the approximations of the mean and standard deviation, respectively. 
that provides approximations for the mean, given by (2.40) with $M \rightarrow+\infty$, is exactly $0 \leq t<50$. To this end, its sufficient to study the first series defined in (2.40), since the analysis of the second series is similar. Taking into account expression (3.7) for the statistical moments of $R V \lambda$, it is clear that series is made up only of non-negative terms for all $m \geq 0$, and it has the following form

$$
\sum_{m \geq 0} \hat{\delta}_{m}(t), \quad \hat{\delta}_{m}(t):=\frac{\mathbb{E}\left[\lambda^{2 m}\right]}{\Gamma(2 \alpha m+1)} t^{2 \alpha m},
$$

Using the Stirling's approximation (3.13) and applying the ratio test, observe that

$$
\begin{aligned}
& \lim _{m \rightarrow+\infty} \frac{\hat{\delta}_{m+1}(t)}{\hat{\delta}_{m}(t)}=\lim _{m \rightarrow+\infty} \frac{(2 m+2)(2 m+1) \sigma^{2}}{2(m+1)} \frac{\Gamma(2 \alpha m+1)}{\Gamma(2 \alpha(m+1)+1)} t^{2 \alpha} \\
& =\frac{\sigma^{2} t^{2 \alpha}}{2} \lim _{m \rightarrow+\infty}\left(\frac{(2 m+2)(2 m+1)}{m+1}\right) \\
& \cdot\left(\lim _{m \rightarrow+\infty} \frac{\Gamma(2 \alpha m+1)}{\Gamma(2 \alpha(m+1)+1)}\right) \\
& =\frac{\sigma^{2} t^{2 \alpha}}{2} \lim _{m \rightarrow+\infty}\left(\frac{(2 m+2)(2 m+1)}{m+1}\right) \\
& \cdot\left(\lim _{m \rightarrow+\infty} \frac{(2 \alpha m)^{2 \alpha m} \mathrm{e}^{-2 \alpha m} \sqrt{2 \pi(2 \alpha m)}}{(2 \alpha(m+1))^{2 \alpha(m+1)} \mathrm{e}^{-2 \alpha(m+1)} \sqrt{2 \pi(2 \alpha(m+1))}}\right) \\
& =\frac{\sigma^{2} t^{2 \alpha}}{2} \lim _{m \rightarrow+\infty}\left(\frac{(2 m+2)(2 m+1)}{m+1}\right)\left(\lim _{m \rightarrow+\infty} \frac{1}{(2 \alpha(m+1))^{2 \alpha}}\right) \\
& =\frac{\sigma^{2} t^{2 \alpha}}{2(2 \alpha)^{2 \alpha}} \lim _{m \rightarrow+\infty}\left(\frac{(2 m+2)(2 m+1)}{(m+1)^{2 \alpha+1}}\right) \\
& =\left\{\begin{array}{ccc}
0 & \text { if } & \alpha>1 / 2, \\
+\infty & \text { if } & \alpha<1 / 2, \\
2 \sigma^{2} t & \text { if } & \alpha=1 / 2 .
\end{array}\right.
\end{aligned}
$$

Therefore, according to the ratio test, if $\alpha<1 / 2$ the domain of convergence of the first series of (2.40) (and hence of the full series (2.40)) is $t>0$; if $\alpha>1 / 2$ there is no convergence for all $t>0$, and if $\alpha=1 / 2$ the domain of convergence is $0<t<1 /\left(2 \sigma^{2}\right)$. Thus, in this latter case if $\sigma=1 / 10$, such as it has been chosen in our numerical experiments, the domain of convergence of the series (2.40) (with 
$M \rightarrow+\infty)$, defining the approximations of the mean is $0 \leq t<50$. This fully agrees with the results shown in Figure 3.4 and Figure 3.5.

Example 3.7 This example illustrates Case II, corresponding to $\alpha=1.2 \in] 1,2]$, when diffusion coefficient $\lambda$ is an unbounded $R V$. Let us consider the random fractional IVP (3.1), where

- $\beta_{0}$ and $\beta_{1}$ are Gamma RVs of mean $1 / 2$ and variance $1 / 2$, i.e. $\beta_{0} \sim$ $G a(1 / 2 ; 1)$.

- $\gamma$ is a Gaussian $R V$ of mean $1 / 2$ and variance $1 / 2, \lambda \sim N\left(1 / 2 ;(\sqrt{2} / 2)^{2}\right)$.

- $\lambda$ is an Exponential $R V$ with mean $1 / 6$ and variance $1 / 36, \lambda \sim \operatorname{Exp}(6)$.

We assume that all input data $\beta_{0}, \beta_{1}, \gamma$ and $\lambda$ are mutually independent $R V s$. Hence, hypothesis $\hat{\mathbf{H}} \mathbf{1}$ is fulfilled. Since $\lambda$ is an Exponential $R V$, it is an unbounded and, by Example 3.3, it satisfies hypothesis $\hat{\mathbf{H} 2}$ with $p=1, \mathcal{H}=1 / 3$ and $\eta=\sqrt{2} / 6$. Therefore, as $p=1<1.2=\alpha$ by Theorem 3.2, it is known that the random generalized power series solution $Y(t)$, given by $(3.25)$, is m.s. convergent for all $t \geq 0$. As a consequence, the approximations for that for the mean and the variance (or standard deviation), given by (3.29)-(3.31), respectively, will converge for all $t \geq 0$. Approximations for these statistical moments are shown in Figure 3.6 using the following orders of truncation $M=5,7,10,12,15$. We observe the convergence over the whole intervals. In Figure 3.7, we show an approximation to the correlation coefficient function associated to the solution SP. This surface has been built taking $M=20$ in the following expression

$$
\rho_{Y_{M}}(t, s)=\frac{\mathbb{C}_{Y_{M}, Y_{M}}(t, s)}{\sqrt{\mathbb{V}\left[Y_{M}(t)\right]} \times \sqrt{\mathbb{V}\left[Y_{M}(s)\right]}} .
$$

This function measures the lineal statistical dependence between the approximations $Y_{M}(s)$ and $Y_{M}(t)$ in two different time instants $s$ and $t$. From Figure 3.7, we can observe that linear statistical interdependence is stronger in points located about the diagonal $(t, t)$. For $M$ fixed, this means that $R V Y_{M}(s)$ can be approximated by a linear function of $Y_{M}(t)$ when $s$ and $t$ are close. 

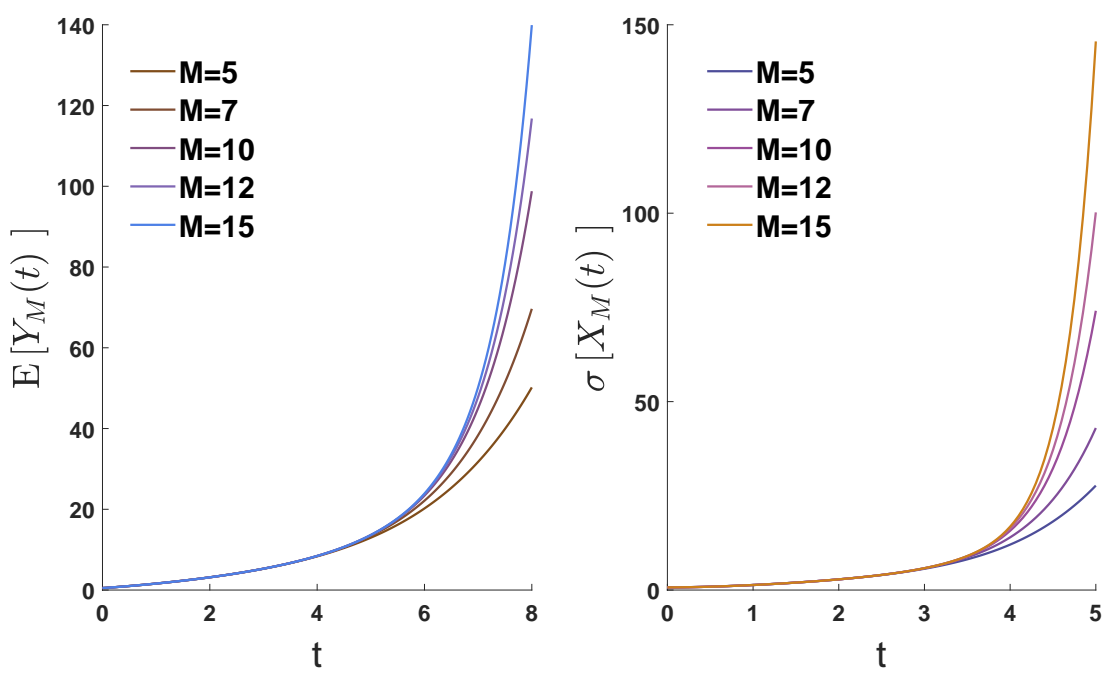

Figure 3.6: Approximations of the mean (left) and the standard deviation (right) of the solution SP to the random fractional IVP (3.1) with $\alpha=1.2$ (Case II) using different orders of truncation $M$ over the time intervals $[0,8]$ and $[0,5]$, respectively in the context of Example 3.7 .

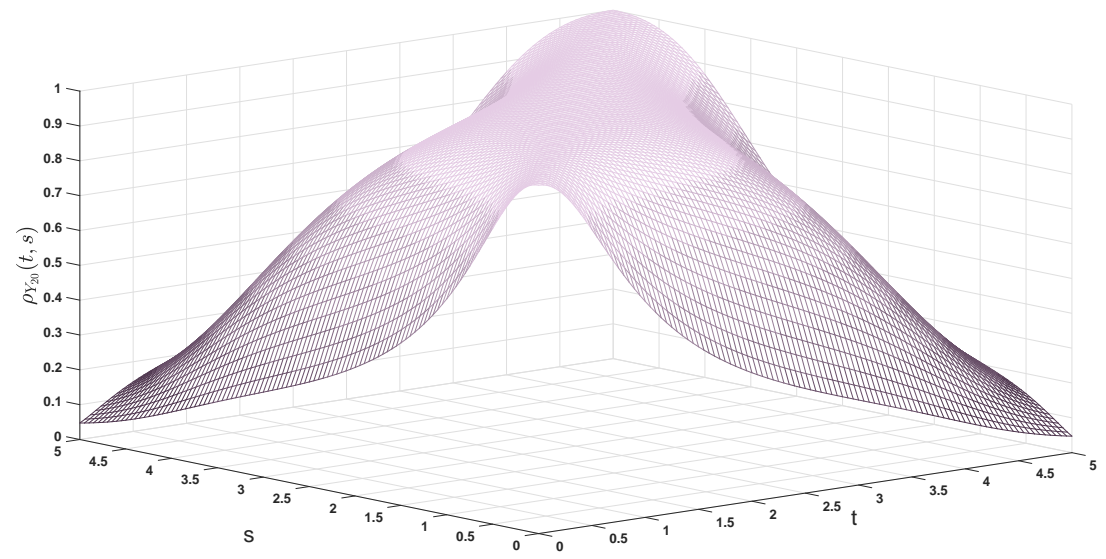

Figure 3.7: Approximations of the correlation coefficient associated to the solution SP to the random fractional IVP (3.1) with $\alpha=1.2$ (Case II) taking as order of truncation $M=20$ in the context of Example 3.7. 


\subsection{Conclusions}

In this chapter we have extended some results recently obtained for the random linear fractional differential equation using the m.s. calculus and the random Caputo derivative. We have constructed a solution SP for that class of equations by means of a random generalized power series. Furthermore, we have given mild conditions in order to guarantee its m.s. convergence. Afterwards, we have provided closed-form expressions for approximations of its main statistical functions (mean, variance, covariance and cross-covariance). The analysis permits to enlarge the family of RVs playing the role of diffusion coefficient for that class of fractional differential equation. Specifically, significant unbounded RVs such as Gaussian and Exponential are included in our hypotheses.

\section{Chapter published}

The results of this chapter have been published in [27]. With regard to this paper, the $\mathrm{PhD}$ candidate has contributed by working in its complete development with more emphasis on the construction of the solution, proving its mean square convergence, computing the main statistical moments and preparing numerical examples. 


\section{Chapter}

\section{4}

A full probabilistic solution of the random linear fractional differential equation via the Random Variable Transformation technique

This chapter is devoted to obtain the first probability density function of the randomized fractional linear non-homogeneous differential equation studied in the previous Chapters with $\alpha \in] 0,1]$. To conduct our analysis, we take advantage of the Random Variable Transformation technique to construct approximations of the first probability density function of the solution stochastic process from a suitable infinite series representation. Then, we prove these approximations do converge to the exact density function assuming mild conditions on random input parameters. Our theoretical findings are illustrated through two numerical examples. 


\subsection{Introduction}

The probability density function (PDF) of a RV is, as its name suggests, a mathematical function which defines entirely this RV and, consequently, all its statistical information. Generalizing this concept through the SPs, if $y(t, \omega)$ is a SP, its associated 1-PDF, which is the PDF of $y(t, \omega)$ for a fixed $t$, allows us to obtain a full probabilistic description of $y(t, \omega)$. Even more, if $y(t, \omega)$ is a SP that describes a real phenomena, its associated 1-PDF provides a complete statistical description of the process.

For instance, from 1-PDF, one can compute all one-dimensional statistical moments at every time instant $t$,

$$
\mathbb{E}\left[(y(t, \omega))^{k}\right]=\int_{-\infty}^{\infty}(y(t))^{k} f_{1}(y, t) \mathrm{d} y, \quad k=1,2, \ldots, \quad \omega \in \Omega .
$$

In particular, the mean $\mu_{y}(t)=\mathbb{E}[y(t, \omega)]$ and the variance $\sigma_{y}^{2}(t)=\mathbb{V}[y(t, \omega)]=$ $\mathbb{E}\left[(y(t, \omega))^{2}\right]-\left(\mu_{y}(t)\right)^{2}$. Furthermore, the 1-PDF allows us to calculate the probability that the solution lies in a particular interval of interest at each time instant $\hat{t}$ fixed, i.e.,

$$
\mathbb{P}[a \leq y(\hat{t}, \omega) \leq b]=\int_{a}^{b} f_{1}(y, \hat{t}) \mathrm{d} y
$$

In this chapter the 1-PDF of the solution SP of the randomized fractional linear differential equation obtained in Chapter 2 is computed. Mild conditions of the random input parameters will be consider in order to guarantee the convergence of the 1-PDF. Although the computation of the 1-PDF of different classes of RDEs has been dealt in the extant literature (see [53, 69, 33, 35] and references therein), to the best of our knowledge, this interesting problem is not been addressed yet.

First of all, let us remind the IVP studied in Chapter 2

$$
\left.\begin{array}{cl}
\left({ }^{C} D_{0}^{\alpha} y\right)(t, \omega)-\lambda(\omega) y(t, \omega) & =\gamma(\omega), \quad 0<\alpha \leq 1, \quad t>0, \\
y(0, \omega) & =\beta_{0}(\omega),
\end{array}\right\}
$$


In (4.3), the initial condition $\beta_{0}(\omega)$, the forcing term $\gamma(\omega)$ and the diffusion coefficient $\lambda(\omega)$ are assumed to be absolutely continuous RVs defined on a common complete probability space $(\Omega, \mathcal{F}, \mathbb{P})$. For sake of clarity, hereinafter we will denote by $\mathcal{D}_{\mathbf{X}}$ the domain of any random vector, say $\mathbf{X}(\omega)$, in particular, $\mathcal{D}_{\left(\beta_{0}, \gamma, \lambda\right)}$ will stand for the domain of input random vector $\mathbf{X}(\omega)=\left(\beta_{0}(\omega), \gamma(\omega), \lambda(\omega)\right)$ and $f_{\beta_{0}, \gamma, \lambda}\left(\beta_{0}, \gamma, \lambda\right)$ will denote its joint PDF.

According to Chapter 2 equation (2.29), the solution SP of random fractional IVP (4.3) can be represented by

$$
y(t, \omega)=\beta_{0}(\omega) S_{1}(t, \alpha ; \lambda(\omega))+\gamma(\omega) S_{2}(t, \alpha ; \lambda(\omega)),
$$

being

$$
\begin{aligned}
& S_{1}(t, \alpha ; \lambda(\omega))=\sum_{m=0}^{\infty} \frac{(\lambda(\omega))^{m}}{\Gamma(\alpha m+1)} t^{\alpha m} \\
& S_{2}(t, \alpha ; \lambda(\omega))=\sum_{m=0}^{\infty} \frac{(\lambda(\omega))^{m}}{\Gamma(\alpha(m+1)+1)} t^{\alpha(m+1)} .
\end{aligned}
$$

Remark 4.1 As it can be observed in (4.4) (4.5), $S_{1}(t, \alpha ; \lambda(\omega))$ and $S_{2}(t, \alpha ; \lambda(\omega))$, and thus the solution $y(t, \omega)$, can be represented as a random series in both parameters $\lambda(\omega), \omega \in \Omega$, and $t$. Taking into account the uniqueness of the solution of random IVP (4.3), both series expansions match (as powers of $t$ and as powers of $\lambda(\omega), \omega \in \Omega$ ). In addition, it can be proved by applying the ratio test together with Stirling's formula that both series converge in $\mathbb{R}$ for all $\omega \in \Omega$, see (2.36). Therefore, there is uniform convergence in every closed set contained in $\mathbb{R}$.

As the solution $y(t, \omega)$ of random IVP (4.3) is given by a linear combination of infinite series (4.4)-(4.5), to compute its $1-\mathrm{PDF}, f_{1}(y, t)$, from a computational point of view it is convenient to consider its approximation via the truncation of both series

$$
y^{M}(t, \omega)=\beta_{0}(\omega) S_{1}^{M}(t, \alpha ; \lambda(\omega))+\gamma(\omega) S_{2}^{M}(t, \alpha ; \lambda(\omega)),
$$


where

$$
\begin{aligned}
& S_{1}^{M}(t, \alpha ; \lambda(\omega))=\sum_{m=0}^{M} \frac{(\lambda(\omega))^{m}}{\Gamma(\alpha m+1)} t^{\alpha m} \\
& S_{2}^{M}(t, \alpha ; \lambda(\omega))=\sum_{m=0}^{M} \frac{(\lambda(\omega))^{m}}{\Gamma(\alpha(m+1)+1)} t^{\alpha(m+1)} .
\end{aligned}
$$

Then, we will first obtain the 1-PDF, $f_{1}^{M}(y, t)$, of the truncated solution $y^{M}(t, \omega)$, and second, we will prove that $f_{1}^{M}(y, t)$ converges to $f_{1}(y, t)$ as $M$ increases, under certain conditions on the input random vector $\left(\beta_{0}(\omega), \gamma(\omega), \lambda(\omega)\right)$ that will be specified later.

To compute $f_{1}^{M}(y, t)$, Random Variable Transformation method (RVT) will be applied. This technique allows us to obtain the PDF of a random vector that results from mapping of another random vector whose PDF is known. For the sake of completeness in the presentation and notation, down below we state a multidimensional version of RVT method.

Theorem 4.1 (Random Variable Transformation method) [109, page 25]. Let us consider $\mathbf{X}=\left(X_{1}, \ldots, X_{n}\right)$ and $\mathbf{Z}=\left(Z_{1}, \ldots, Z_{n}\right)$ two $n$-dimensional absolutely continuous random vectors defined on a common probability space $(\Omega, \mathcal{F}, \mathbb{P})$. Let $\mathbf{r}: \mathbb{R}^{n} \rightarrow \mathbb{R}^{n}$ be a one-to-one deterministic transformation of $\mathbf{X}$ into $\mathbf{Z}$, i.e., $\mathbf{Z}=\mathbf{r}(\mathbf{X})$. Assume that $\mathbf{r}$ is continuous in $\mathbf{X}$ and has continuous partial derivatives with respect to each $X_{i}, 1 \leq i \leq n$. Then, if $f_{\mathbf{X}}(\mathbf{x})$ denotes the joint probability density function of random vector $\mathbf{X}$, and $\mathbf{s}=\mathbf{r}^{-1}=\left(s_{1}\left(z_{1}, \ldots, z_{n}\right), \ldots, s_{n}\left(z_{1}, \ldots, z_{n}\right)\right)$ represents the inverse mapping of $\mathbf{r}=\left(r_{1}\left(x_{1}, \ldots, x_{n}\right), \ldots, r_{n}\left(x_{1}, \ldots, x_{n}\right)\right)$, the joint probability density function of random vector $\mathbf{Z}$ is given by

$$
f_{\mathbf{Z}}(\mathbf{z})=f_{\mathbf{X}}(\mathbf{s}(\mathbf{z}))|J|
$$

where $|J|$, which is assumed to be different from zero, is the absolute value of the Jacobian defined by the determinant

$$
J=\operatorname{det}\left(\frac{\partial \mathbf{s}^{\top}}{\partial \mathbf{z}}\right)=\operatorname{det}\left(\begin{array}{ccc}
\frac{\partial s_{1}\left(z_{1}, \ldots, z_{n}\right)}{\partial z_{1}} & \cdots & \frac{\partial s_{n}\left(z_{1}, \ldots, z_{n}\right)}{\partial z_{1}} \\
\vdots & \ddots & \vdots \\
\frac{\partial s_{1}\left(z_{1}, \ldots, z_{n}\right)}{\partial z_{n}} & \cdots & \frac{\partial s_{n}\left(z_{1}, \ldots, z_{n}\right)}{\partial z_{n}}
\end{array}\right)
$$


The following technical remark will be useful later to legitimate the application of RVT technique in an appropriate neighbourhood where the 1-PDF of the solution SP will be computed.

Remark 4.2 Let $\alpha \in] 0,1]$, taking into account expression (4.7) it is clear that $S_{1}^{M}(0, \alpha ; \lambda(\omega))=1 \neq 0, \forall \omega \in \Omega, \forall M \geq 0$ integer. From Remark $4.1 S_{1}(t, \alpha ; \lambda(\omega))$ can be represented via a power series in $t$ and $\lambda(\omega), \forall \omega \in \Omega$. Since $S_{1}^{M}(t, \alpha ; \lambda(\omega))$ is a polynomial in both variables $t$ and $\lambda(\omega)$, then is a continuous function in these variables. Therefore, there exists a neighbourhood, $\mathcal{N}(0,0(\omega))$ of $(t=0, \lambda(\omega)=$ $0(\omega))$ such that

$$
0<m_{S_{1}}<\left|S_{1}^{M}(t, \alpha ; \lambda(\omega))\right|, \quad \forall(t, \lambda(\omega)) \in \mathcal{N}(0,0(\omega)), \quad \forall \omega \in \Omega, \quad \forall M \in \mathbb{Z}^{+} .
$$

On the other hand, by Remark 4.1 both series $S_{i}(t, \alpha ; \lambda(\omega)), i=1,2$, converge uniformly in every closed set of $\mathbb{R}$. Then, we can take, without loss of generality, the closure of neighbourhood $\mathcal{N}(0,0(\omega)), \mathcal{N}^{*}(0,0(\omega))$. This guarantees the existence of positive constants $M_{S_{i}}, i=1,2$, such that

$$
\left|S_{i}^{M}(t, \alpha ; \lambda(\omega))\right|<M_{S_{i}}
$$

for all $(t, \lambda(\omega)) \in \mathcal{N}^{*}(0,0(\omega)) \subset \mathcal{N}(0,0(\omega)), \omega \in \Omega$ and $M \in \mathbb{Z}^{+}$. Notice that in Remark 4.2, we have taken the neighbourhood of point $(t, \lambda(\omega))=(0,0(\omega))$. This choice is motivated by the expression of $S_{1}(t, \alpha ; \lambda(\omega))$ given in $(4.5)$, where, as it has been previously pointed, one can observe that is a power series in $\lambda(\omega)$, $\forall \omega \in \Omega$. Then, it is centred in $\lambda(\omega)=0(\omega), \forall \omega \in \Omega$.

This chapter is organized as follows. In Section 4.2 the 1-PDF of the truncated solution SP given by (4.6) is constructed by applying the RVT technique stated in Theorem 4.1. Then, we prove the convergence of this function to the 1-PDF of the exact solution of RFDE (4.3) under suitable hypotheses on random inputs. Section 4.3 is devoted to show several examples that illustrate our theoretical findings. Finally, in Section 4.4 our main conclusions are drawn. 


\subsection{Main result: Computing approximations of the 1-PDF of the solution stochastic process}

In this section, we will first compute the 1-PDF $f_{1}^{M}(y, t)$ by taking advantage of RVT technique. With this goal, let $\alpha \in] 0,1], t>0, \omega \in \Omega$ fixed, such that $(t, \lambda(\omega))$ belongs to the neighbourhood $\mathcal{N}^{*}(0,0(\omega))$ introduced in Remark 4.2 and let us apply Theorem 4.1 with the following identification: $\mathbf{X}(\omega)=\left(\beta_{0}(\omega), \gamma(\omega), \lambda(\omega)\right)$, $\mathbf{Z}(\omega)=\left(Z_{1}(\omega), Z_{2}(\omega), Z_{3}(\omega)\right)$ and the mapping $\mathbf{r}: \mathbb{R}^{3} \longrightarrow \mathbb{R}^{3}$ whose components $\left(r_{1}, r_{2}, r_{3}\right)$ are defined as

$$
\begin{aligned}
& z_{1}=r_{1}\left(\beta_{0}, \gamma, \lambda\right)=\beta_{0} S_{1}^{M}(t, \alpha ; \lambda)+\gamma S_{2}^{M}(t, \alpha ; \lambda), \\
& z_{2}=r_{2}\left(\beta_{0}, \gamma, \lambda\right)=\gamma \\
& z_{3}=r_{3}\left(\beta_{0}, \gamma, \lambda\right)=\lambda .
\end{aligned}
$$

The inverse mapping $\mathbf{s}$ of $\mathbf{r}$ is given by

$$
\begin{aligned}
& \beta_{0}=s_{1}\left(z_{1}, z_{2}, z_{3}\right)=\frac{z_{1}-z_{2} S_{2}^{M}\left(t, \alpha ; z_{3}\right)}{S_{1}^{M}\left(t, \alpha ; z_{3}\right)} \\
& \gamma=s_{2}\left(z_{1}, z_{2}, z_{3}\right)=z_{2} \\
& \lambda=s_{3}\left(z_{1}, z_{2}, z_{3}\right)=z_{3}
\end{aligned}
$$

From (4.10), it is known that $\left|S_{1}^{M}(t, \alpha ; \lambda(\omega))\right| \neq 0, \forall(t, \lambda(\omega)) \in \mathcal{N}^{*}(0,0(\omega))$, and thus the absolute value of the Jacobian of inverse mapping $\mathbf{s}$ is well-defined and non-zero

$$
|J|=\left|\frac{\partial s_{1}}{\partial z_{1}}\right|=\left|\frac{1}{S_{1}^{M}\left(t, \alpha ; z_{3}\right)}\right| \neq 0 .
$$

As a consequence, by applying Theorem 4.1, the PDF of random vector $\mathbf{Z}(\omega)$ is given by

$$
f_{Z_{1}, Z_{2}, Z_{3}}\left(z_{1}, z_{2}, z_{3}\right)=f_{\beta_{0}, \gamma, \lambda}\left(\frac{z_{1}-z_{2} S_{2}^{M}\left(t, \alpha ; z_{3}\right)}{S_{1}^{M}\left(t, \alpha ; z_{3}\right)}, z_{2}, z_{3}\right)\left|\frac{1}{S_{1}^{M}\left(t, \alpha ; z_{3}\right)}\right| .
$$

Finally, marginalizing with respect to $Z_{2}(\omega)=\gamma(\omega)$ and $Z_{3}(\omega)=\lambda(\omega)$ and taking $t>0$ arbitrary, the 1-PDF of the truncated solution SP, $y^{M}(t, \omega)$, of random 
fractional IVP (4.3) is obtained

$$
f_{1}^{M}(y, t)=\int_{\mathcal{D}(\gamma, \lambda)} f_{\beta_{0}, \gamma, \lambda}\left(\frac{y-\gamma S_{2}^{M}(t, \alpha ; \lambda)}{S_{1}^{M}(t, \alpha ; \lambda)}, \gamma, \lambda\right)\left|\frac{1}{S_{1}^{M}(t, \alpha ; \lambda)}\right| \mathrm{d} \lambda \mathrm{d} \gamma
$$

Now, we address the proof of the convergence of the 1-PDF, $f_{1}^{M}(y, t)$, given in (4.12), to the 1-PDF of the exact solution, $f_{1}(y, t)$, given by

$$
f_{1}(y, t)=\int_{\mathcal{D}(\gamma, \lambda)} f_{\beta_{0}, \gamma, \lambda}\left(\frac{y-\gamma S_{2}(t, \alpha ; \lambda)}{S_{1}(t, \alpha ; \lambda)}, \gamma, \lambda\right)\left|\frac{1}{S_{1}(t, \alpha ; \lambda)}\right| \mathrm{d} \lambda \mathrm{d} \gamma,
$$

where $S_{1}(t, \alpha ; \lambda)$ and $S_{2}(t, \alpha ; \lambda)$ are defined in $(4.5)$, for each $(y, t) \in \mathbb{R} \times[0, \infty[$ fixed, as $M \rightarrow \infty$, i.e., $\lim _{M \rightarrow \infty} f_{1}^{M}(y, t)=f_{1}(y, t)$ for each $(y, t) \in \mathbb{R} \times[0, \infty[$ fixed with $t$ such that $(t, \lambda(\omega)) \in \mathcal{N}^{*}(0,0(\omega))$. To this end, henceforth the following hypotheses will be assumed

J1: Inputs parameters $\beta_{0}(\omega), \gamma(\omega)$ and $\lambda(\omega)$ of random IVP (4.3) are independent RVs defined in a common complete probability space $(\Omega, \mathcal{F}, \mathbb{P})$.

J2: The expectation of the absolute value of RV defining the non-homogeneous term of random $\operatorname{IVP}(4.3)$ is finite, i.e., $\mathbb{E}[|\gamma(\omega)|]=C<\infty$.

J3: The PDF of the random initial condition $f_{\beta_{0}}\left(\beta_{0}\right)$ is Lipschitz continuous in $\mathbb{R}$, i.e.,

$$
\exists L_{0}>0: \quad\left|f_{\beta_{0}}\left(\beta_{0,1}\right)-f_{\beta_{0}}\left(\beta_{0,2}\right)\right| \leq L_{0}\left|\beta_{0,1}-\beta_{0,2}\right|, \quad \forall \beta_{0,1}, \beta_{0,2} \in \mathbb{R} .
$$

Now, we comment about the generality of above assumptions from a practical point of view. Hypothesis $\mathbf{J} \mathbf{1}$ is just imposed to simplify the subsequent development. In particular, it allows us to apply the following factorization $f_{\beta_{0}, \gamma, \lambda}\left(\beta_{0}, \gamma, \lambda\right)=$ $f_{\beta_{0}}\left(\beta_{0}\right) f_{\gamma}(\gamma) f_{\lambda}(\lambda)$. As a consequence, expression (4.12) can be written as follows

$$
f_{1}^{M}(y, t)=\int_{\mathcal{D}(\gamma)} \int_{\mathcal{D}(\lambda)} f_{\beta_{0}}\left(\frac{y-\gamma S_{2}^{M}(t, \alpha ; \lambda)}{S_{1}^{M}(t, \alpha ; \lambda)}\right) f_{\gamma}(\gamma) f_{\lambda}(\lambda)\left|\frac{1}{S_{1}^{M}(t, \alpha ; \lambda)}\right| \mathrm{d} \lambda \mathrm{d} \gamma
$$


Independence among random inputs is a usual assumption in the analysis of RFDEs (see $[24,21]$ for instance), moreover it is also natural from an applied standpoint since random inputs usually have not direct relationship. Hypothesis J2 is equivalent to assume that $\gamma \in \mathrm{L}^{1}(\Omega)$, being $\mathrm{L}^{1}(\Omega)$ the biggest of $\mathrm{L}^{p}(\Omega)$-Banach spaces [87], so this is a very general assumption since to this space belong the majority of important RVs (Gaussian, Gamma, Beta, etc.). Finally, it is worth mentioning that $\mathbf{J 3}$ only affects the random initial condition $\beta_{0}$ and, again it is fulfilled for most of relevant RVs (Gaussian, Gamma, Beta, etc.,) since it is easy to check that the first derivative of the PDF of RVs is bounded, hence it is Lipschitz.

Let $\varepsilon>0$ arbitrary and let us consider the following development addressed to prove the convergence of $f_{1}^{M}(y, t)$ to $f_{1}(y, t)$ as $M$ increases for every $(y, t) \in \mathbb{R} \times[0, \infty[$ fixed, where $t>0$ is such that $(t, \lambda(\omega)) \in \mathcal{N}^{*}(0,0(\omega))$ being $\mathcal{N}^{*}(0,0(\omega))$ the neighbourhood whose existence is guaranteed by Remark 4.2 .

$$
\begin{aligned}
& \left|f_{1}^{M}(y, t)-f_{1}(y, t)\right| \\
= & \left|\int_{\mathcal{D}(\gamma)} \int_{\mathcal{D}(\lambda)} f_{\beta_{0}}\left(\frac{y-\gamma S_{2}^{M}(t, \alpha ; \lambda)}{S_{1}^{M}(t, \alpha ; \lambda)}\right) f_{\gamma}(\gamma) f_{\lambda}(\lambda)\right| \frac{1}{S_{1}^{M}(t, \alpha ; \lambda)} \mid \mathrm{d} \lambda \mathrm{d} \gamma \\
& -\int_{\mathcal{D}(\gamma)} \int_{\mathcal{D}(\lambda)} f_{\beta_{0}}\left(\frac{y-\gamma S_{2}(t, \alpha ; \lambda)}{S_{1}(t, \alpha ; \lambda)}\right) f_{\gamma}(\gamma) f_{\lambda}(\lambda)\left|\frac{1}{S_{1}(t, \alpha ; \lambda)}\right| \mathrm{d} \lambda \mathrm{d} \gamma \mid \\
\leq & \int_{\mathcal{D}(\gamma)} \int_{\mathcal{D}(\lambda)}\left|f_{\beta_{0}}\left(\frac{y-\gamma S_{2}^{M}(t, \alpha ; \lambda)}{S_{1}^{M}(t, \alpha ; \lambda)}\right)\right| \frac{1}{S_{1}^{M}(t, \alpha ; \lambda)} \mid \\
& -f_{\beta_{0}}\left(\frac{y-\gamma S_{2}^{M}(t, \alpha ; \lambda)}{S_{1}^{M}(t, \alpha ; \lambda)}\right)\left|\frac{1}{S_{1}(t, \alpha ; \lambda)}\right| \\
& +f_{\beta_{0}}\left(\frac{y-\gamma S_{2}^{M}(t, \alpha ; \lambda)}{S_{1}^{M}(t, \alpha ; \lambda)}\right)\left|\frac{1}{S_{1}(t, \alpha ; \lambda)}\right| \\
& -f_{\beta_{0}}\left(\frac{y-\gamma S_{2}(t, \alpha ; \lambda)}{S_{1}(t, \alpha ; \lambda)\left|\frac{1}{S_{1}(t, \alpha ; \lambda)}\right| \mid f_{\gamma}(\gamma) f_{\lambda}(\lambda) \mathrm{d} \lambda \mathrm{d} \gamma}\right. \\
\leq & \int_{\mathcal{D}(\gamma)} \int_{\mathcal{D}(\lambda)}[\underbrace{\left.f_{\beta_{0}\left(\frac{y-\gamma}{S_{2}}(t, \alpha ; \lambda)\right.}^{S_{1}^{M}(t, \alpha ; \lambda)}\right)}_{(\mathrm{I})}|| \frac{1}{f_{1}^{M}(t, \alpha ; \lambda)}|-| \frac{1}{S_{1}(t, \alpha ; \lambda)}||
\end{aligned}
$$




$$
+\underbrace{\left\lfloor f_{\beta_{0}}\left(\frac{y-\gamma S_{2}^{M}(t, \alpha ; \lambda)}{S_{1}^{M}(t, \alpha ; \lambda)}\right)-f_{\beta_{0}}\left(\frac{y-\gamma S_{2}(t, \alpha ; \lambda)}{S_{1}(t, \alpha ; \lambda)}\right) \mid\right.}_{(\mathrm{III})} \underbrace{\left.\mid \frac{1}{S_{1}(t, \alpha ; \lambda)}\right\rfloor}_{(\mathrm{IV})}\rfloor
$$

$f_{\gamma}(\gamma) f_{\lambda}(\lambda) \mathrm{d} \lambda \mathrm{d} \gamma$

Now, we will obtain some adequate bounds for the terms (I)-(IV) of (4.15), that will be used later. Let us start with bound (I). According to Remark 4.2, hypothesis J3, and denoting $F_{0}=f_{\beta_{0}}(0)$ without loss of generality, one obtains

$$
\begin{aligned}
f_{\beta_{0}}\left(\frac{y-\gamma S_{2}^{M}(t, \alpha ; \lambda)}{S_{1}^{M}(t, \alpha ; \lambda)}\right) & \leq\left|f_{\beta_{0}}\left(\frac{y-\gamma S_{2}^{M}(t, \alpha ; \lambda)}{S_{1}^{M}(t, \alpha ; \lambda)}\right)-f_{\beta_{0}}(0)\right|+F_{0} \\
& \leq L_{0}\left|\frac{y-\gamma S_{2}^{M}(t, \alpha ; \lambda)}{S_{1}^{M}(t, \alpha ; \lambda)}\right|+F_{0} \\
& \leq L_{0} \frac{|y|+|\gamma|\left|S_{2}^{M}(t, \alpha ; \lambda)\right|}{\left|S_{1}^{M}(t, \alpha, \lambda)\right|}+F_{0} \\
& \leq \frac{L_{0}}{m_{S_{1}}}\left(|y|+|\gamma| M_{S_{2}}\right)+F_{0} .
\end{aligned}
$$

Let us obtain a suitable bound for the term (II). On the one hand, by expression (4.10) of Remark 4.2, we know that $0<m_{S_{1}} \leq\left|S_{1}(t, \alpha ; \lambda)\right|$ for all for all $(t, \lambda) \in$ $\mathcal{N}^{*}(0,0(\omega))$ and $\left.\left.\alpha \in\right] 0,1\right]$. On the other hand, let us recall that in agreement with Remark 4.1 it is known that $S_{i}^{M}(t, \alpha ; \lambda)$, converges uniformly to $S_{i}(t, \alpha ; \lambda)$ for all $(t, \lambda) \in \mathcal{N}^{*}(0,0(\omega))$ and $\left.\left.\alpha \in\right] 0,1\right], i=1,2$, i.e., for all $\varepsilon_{i}>0$, there exists $M_{0}$, which may depends on $\varepsilon_{i}$, such that

$$
\left|S_{i}(t, \alpha ; \lambda)-S_{i}^{M}(t, \alpha ; \lambda)\right|<\varepsilon_{i}, \quad i=1,2, \quad \forall M \geq M_{0} .
$$

Therefore, applying (4.17) for $i=1$, one gets

$$
\begin{aligned}
\left\|\frac{1}{S_{1}^{M}(t, \alpha ; \lambda)}|-| \frac{1}{S_{1}(t, \alpha ; \lambda)}\right\| & \leq\left|\frac{1}{S_{1}^{M}(t, \alpha ; \lambda)}-\frac{1}{S_{1}(t, \alpha ; \lambda)}\right| \\
& =\frac{\left|S_{1}(t, \alpha ; \lambda)-S_{1}^{M}(t, \alpha ; \lambda)\right|}{\left|S_{1}^{M}(t, \alpha ; \lambda)\right|\left|S_{1}(t, \alpha ; \lambda)\right|} \\
& \leq \frac{\varepsilon_{1}}{\left(m_{S_{1}}\right)^{2}} .
\end{aligned}
$$


As it shall be seen later on, the term (IV) we will be bounded by

$$
\left|1 / S_{1}(t, \alpha ; \lambda)\right|<1 / m_{S_{1}},
$$

which is straightforwardly derived from expression (4.10) in Remark 4.2. Finally, we will obtain a proper bound to be used for the term (III). To this end, we will apply Remark 4.2, hypothesis $\mathbf{J} 3$ and the convergence of both series $S_{i}(t, \alpha ; \lambda)$, $i=1,2$ (taking $\varepsilon_{2}=\frac{\left(m_{S_{1}}\right)^{3}}{L_{0} M_{S_{1}}} \frac{\varepsilon}{2}>0$ in (4.17)). This yields

$$
\begin{aligned}
& \left|f_{\beta_{0}}\left(\frac{y-\gamma S_{2}^{M}(t, \alpha ; \lambda)}{S_{1}^{M}(t, \alpha ; \lambda)}\right)-f_{\beta_{0}}\left(\frac{y-\gamma S_{2}(t, \alpha ; \lambda)}{S_{1}(t, \alpha ; \lambda)}\right)\right| \\
& \leq L_{0}\left|\frac{y-\gamma S_{2}^{M}(t, \alpha ; \lambda)}{S_{1}^{M}(t, \alpha ; \lambda)}-\frac{y-\gamma S_{2}(t, \alpha ; \lambda)}{S_{1}(t, \alpha ; \lambda)}\right| \\
& =L_{0} \frac{\left|y S_{1}(t, \alpha ; \lambda)-\gamma S_{2}^{M}(t, \alpha ; \lambda) S_{1}(t, \alpha ; \lambda)-y S_{1}^{M}(t, \alpha ; \lambda)+\gamma S_{2}(t, \alpha ; \lambda) S_{1}^{M}(t, \alpha ; \lambda)\right|}{\left|S_{1}(t, \alpha ; \lambda)\right|\left|S_{1}^{M}(t, \alpha ; \lambda)\right|} \\
& =\frac{L_{0}}{\left|S_{1}(t, \alpha ; \lambda)\right|\left|S_{1}^{M}(t, \alpha ; \lambda)\right|} \mid y S_{1}(t, \alpha ; \lambda)-\gamma S_{2}^{M}(t, \alpha ; \lambda) S_{1}(t, \alpha ; \lambda) \\
& -y S_{1}^{M}(t, \alpha ; \lambda)+\gamma S_{2}(t, \alpha ; \lambda) S_{1}^{M}(t, \alpha ; \lambda) \mid \\
& \leq \frac{L_{0}}{\left|S_{1}(t, \alpha ; \lambda)\right|\left|S_{1}^{M}(t, \alpha ; \lambda)\right|}\left[|y|\left|S_{1}(t, \alpha ; \lambda)-S_{1}^{M}(t, \alpha ; \lambda)\right|\right. \\
& \left.+|\gamma|\left|S_{2}(t, \alpha ; \lambda) S_{1}^{M}(t, \alpha ; \lambda)-S_{2}^{M}(t, \alpha ; \lambda) S_{1}(t, \alpha ; \lambda)\right|\right] \\
& \leq \frac{L_{0}}{\left|S_{1}(t, \alpha ; \lambda)\right|\left|S_{1}^{M}(t, \alpha ; \lambda)\right|}\left[|y| \varepsilon_{1}+|\gamma| \mid S_{2}(t, \alpha ; \lambda) S_{1}^{M}(t, \alpha ; \lambda)\right. \\
& -S_{2}(t, \alpha ; \lambda) S_{1}(t, \alpha ; \lambda)+S_{2}(t, \alpha ; \lambda) S_{1}(t, \alpha ; \lambda) \\
& \left.-S_{2}^{M}(t, \alpha ; \lambda) S_{1}(t, \alpha ; \lambda) \mid\right] \\
& \leq \frac{L_{0}}{\left|S_{1}(t, \alpha ; \lambda)\right|\left|S_{1}^{M}(t, \alpha ; \lambda)\right|}\left[|y| \varepsilon_{1}+|\gamma|\left|S_{2}(t, \alpha ; \lambda)\right|\left|S_{1}^{M}(t, \alpha ; \lambda)-S_{1}(t, \alpha ; \lambda)\right|\right. \\
& \left.+\left|S_{1}(t, \alpha ; \lambda)\right|\left|S_{2}(t, \alpha ; \lambda)-S_{2}^{M}(t, \alpha ; \lambda)\right|\right] \\
& \leq \frac{L_{0}}{\left(m_{S_{1}}\right)^{2}}\left[|y| \varepsilon_{1}+|\gamma| M_{S_{2}} \varepsilon_{1}+M_{S_{1}} \varepsilon_{2}\right]=\frac{L_{0}}{\left(m_{S_{1}}\right)^{2}}\left(|y|+|\gamma| M_{S_{2}}\right) \varepsilon_{1}+\frac{m_{S_{1}} \varepsilon}{2} \text {. }
\end{aligned}
$$


Now, we firstly substitute bounds (4.16), (4.18)-(4.20) in (4.15), secondly we apply that $\int_{\mathcal{D}(\lambda)} f_{\lambda}(\lambda) \mathrm{d} \lambda=1$ and the expression of expectation operator via an integral as well. This leads

$$
\begin{aligned}
& \left|f_{1}^{M}(y, t)-f_{1}(y, t)\right| \leq \int_{\mathcal{D}(\gamma)} \int_{\mathcal{D}(\lambda)}\left[\left(\frac{L_{0}}{m_{S_{1}}}\left(|y|+|\gamma| M_{S_{2}}\right)+F_{0}\right) \frac{\varepsilon_{1}}{\left(m_{S_{1}}\right)^{2}}\right. \\
& \left.\quad+\left(\frac{L_{0}}{\left(m_{S_{1}}\right)^{2}}\left(|y|+|\gamma| M_{S_{2}}\right) \varepsilon_{1}+\frac{m_{S_{1}} \varepsilon}{2}\right) \frac{1}{m_{S_{1}}}\right] f_{\gamma}(\gamma) f_{\lambda}(\lambda) \mathrm{d} \lambda \mathrm{d} \gamma \\
& =\int_{\mathcal{D}(\gamma)}\left[\left(\frac{2 L_{0}}{\left(m_{S_{1}}\right)^{3}}\left(|y|+|\gamma| M_{S_{2}}\right)+\frac{F_{0}}{\left(m_{S_{1}}\right)^{2}}\right) \varepsilon_{1}+\frac{\varepsilon}{2}\right] f_{\gamma}(\gamma) \mathrm{d} \gamma \\
& =\mathbb{E}\left[\left(\frac{2 L_{0}}{\left(m_{S_{1}}\right)^{3}}\left(|y|+|\gamma| M_{S_{2}}\right)+\frac{F_{0}}{\left(m_{S_{1}}\right)^{2}}\right) \varepsilon_{1}+\frac{\varepsilon}{2}\right] \\
& =\left(\frac{2 L_{0}}{\left(m_{S_{1}}\right)^{3}}\left(|y|+\mathbb{E}[|\gamma|] M_{S_{2}}\right)+\frac{F_{0}}{\left(m_{S_{1}}\right)^{2}}\right) \varepsilon_{1}+\frac{\varepsilon}{2} .
\end{aligned}
$$

Finally, taking $\varepsilon_{1}=\frac{\left(m_{S_{1}}\right)^{3}}{2 L_{0}\left(|y|+C M_{S_{2}}\right)+F_{0} m_{S_{1}}} \frac{\varepsilon}{2}$ and applying hypothesis $\mathbf{J} 2$ in the latter expression, one concludes

$$
\left|f_{1}^{M}(y, t)-f_{1}(y, t)\right| \leq\left(\frac{2 L_{0}}{\left(m_{S_{1}}\right)^{3}}\left(|y|+C M_{S_{2}}\right)+\frac{F_{0}}{\left(m_{S_{1}}\right)^{2}}\right) \varepsilon_{1}+\frac{\varepsilon}{2}=\frac{\varepsilon}{2}+\frac{\varepsilon}{2}=\varepsilon .
$$

Summarizing the following result has been established:

Theorem 4.2 Let us consider random IVP (4.3) and assume that

J1: $\beta_{0}(\omega), \gamma(\omega)$ and $\lambda(\omega)$ are independent $R V$ s defined in a common complete probability space $(\Omega, \mathcal{F}, \mathbb{P})$.

J2: $\mathbb{E}[|\gamma(\omega)|]=C<\infty$.

J3: The PDF $f_{\beta_{0}}\left(\beta_{0}\right)$ of $\beta_{0}(\omega)$ is Lipschitz continuous in $\mathbb{R}$.

Let $S_{1}^{M}(t, \alpha ; \lambda)$ and $S_{2}^{M}(t, \alpha ; \lambda)$ denote the random finite sums given by $(4.7)$. Then, $f_{1}^{M}(y, t)$ defined by (4.14) is the first probability density function of the approximate solution SP (4.6) of random IVP (4.3). Furthermore, for each $(y, t) \in \mathbb{R} \times] 0, \infty[$ 
fixed, $f_{1}^{M}(y, t)$ converges to the first probability density function $f_{1}(y, t)$, given by (4.13), of the exact solution $y(t, \omega)$ defined by $(4.4)-(4.5)$.

\subsection{Numerical examples}

In this section we will show two numerical examples addressed to illustrate our previous theoretical findings. In each example, a variety of probability distributions for input parameters of random fractional IVP (4.3) will be considered.

Example 4.1 Let us assume that the fractional order of Caputo derivative is $\alpha=0.3$, the diffusion coefficient $\lambda(\omega)$ has a Beta distribution with parameters 30 and 40 , i.e., $\lambda(\omega) \sim \operatorname{Be}(30 ; 40)$, the non-homogeneous term $\gamma(\omega)$ is a Gaussian $R V$ with mean 0 and standard deviation $0.1, \gamma(\omega) \sim N(0 ; 0.1)$ and the initial condition $\beta_{0}(\omega)$ has an Exponential distribution with mean $1, \beta_{0}(\omega) \sim \operatorname{Exp}(1)$. In agreement with hypothesis $\boldsymbol{J 1}$, we will assume that $\lambda(\omega), \gamma(\omega)$ and $\beta_{0}(\omega)$ are independent. $A$ direct computation shows that $\mathbb{E}[|\gamma|]=\frac{1}{5 \sqrt{2 \pi}}<\infty$, thus hypothesis J2 holds. Also the PDF of $\beta_{0}(\omega)$ is $f_{\beta_{0}}\left(\beta_{0}\right)=\mathrm{e}^{-\beta_{0}}, \beta_{0}>0$ (and null otherwise), it first derivative satisfies $\left|f_{\beta_{0}}^{\prime}\left(\beta_{0}\right)\right|=\mathrm{e}^{-\beta_{0}}<1$ for all $\beta_{0}>0$, i.e., $f_{\beta_{0}}$ is bounded and hence is Lipschitz. As a result, hypothesis $\boldsymbol{J} 3$ fulfils too.

In Figure 4.1 the approximate 1-PDF, $f_{1}^{M}(y, t)$, given by (4.14) has been plotted for different values of the truncation order $M$ and the time instants $\hat{t}=\{0.1,1,5\}$. From it, one can observe that rapid convergence of approximations. As a measure of the error of these approximations, we have define

$$
e_{M}(\hat{t})=\int_{-\infty}^{\infty}\left|f_{1}^{M}(y, \hat{t})-f_{1}^{M+1}(y, \hat{t})\right| \mathrm{d} y, \quad M \geq 1 \text { integer }
$$

In Table 4.1, we have collected these errors for time instants $\hat{t}=\{0.1,1,5\}$ taking as stopping criterion that $e_{M}(\hat{t}) \leq 10^{-2}$.

In Figure 4.2, we show the mean, $\mu_{y^{M}}(t)$, and the variance, $\sigma_{y^{M}}^{2}(t)$, for different values of truncation order $M$ on the time interval $t \in[0,5]$. In these plots, the convergence of both statistical moments is clearly observed. To account for the quality of approximations of the mean and the variance, we consider the following 

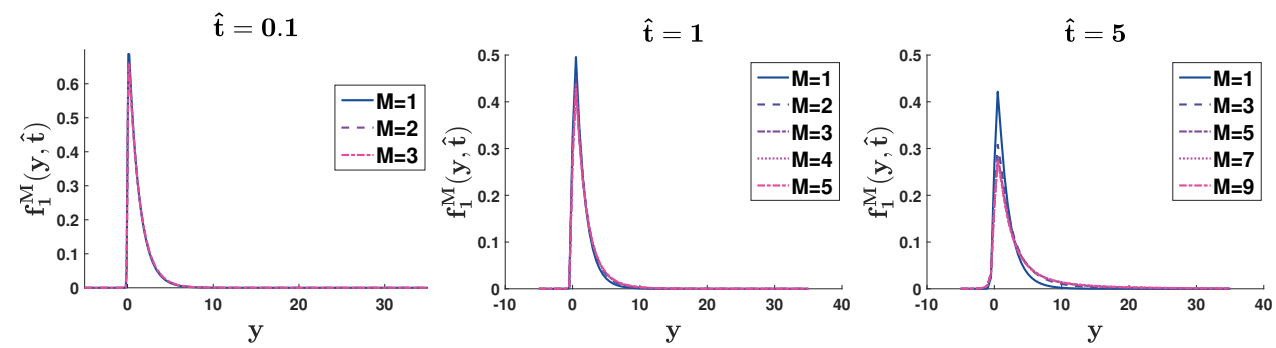

Figure 4.1: Plots of the 1-PDF, $f_{1}^{M}(y, t)$, given by (4.14), for different truncations $M$ at the time instants $\hat{t}=0.1$ (left), $\hat{t}=1$ (center) and $\hat{t}=5$ (right) in Example 4.1.

\begin{tabular}{lcccccccc}
\hline$e_{M}(\hat{t})$ & $\mathrm{M}=1$ & $\mathrm{M}=2$ & $\mathrm{M}=3$ & $\mathrm{M}=4$ & $\mathrm{M}=5$ & $\mathrm{M}=6$ & $\mathrm{M}=7$ & $\mathrm{M}=8$ \\
\hline \hline$\hat{t}=0.1$ & 0.031 & 0.006 & - & - & - & - & - & - \\
\hline$\hat{t}=1$ & 0.094 & 0.040 & 0.019 & 0.009 & - & - & - & - \\
\hline$\hat{t}=5$ & 0.190 & 0.105 & 0.064 & 0.041 & 0.027 & 0.018 & 0.013 & 0.009 \\
\hline
\end{tabular}

Table 4.1: Values of error $e_{M}(\hat{t})$ defined in (4.21) at the time instants $\hat{t}=\{0.1,1,5\}$ in Example 4.1. The truncation order $M$ has been computed so that $e_{M}(\hat{t}) \leq 10^{-2}$.

global error over the whole time interval $[0,5]$

$e_{M}^{\mu}=\int_{0}^{5}\left|\mu_{y^{M}}(t)-\mu_{y^{M+1}}(t)\right| \mathrm{d} t, \quad e_{M}^{\sigma^{2}}=\int_{0}^{5}\left|\sigma_{y^{M}}^{2}(t)-\sigma_{y^{M+1}}^{2}(t)\right| \mathrm{d} t, M \geq 1$ integer.

In agreement with (4.1), the above term $\mu_{y^{M}}(t)$ is computed as

$$
\mu_{y^{M}}(t)=\mathbb{E}\left[y^{M}(t, \omega)\right]=\int_{-\infty}^{\infty} y^{M}(t) f_{1}^{M}(y, t) d y, \quad \omega \in \Omega,
$$

where $y^{M}(t, \omega)$ and $f_{1}^{M}(y, t)$ are given by, (4.6)-(4.7) and (4.14), respectively. Analogously,

$\sigma_{y^{M}}^{2}(t)=\mathbb{E}\left[\left(y^{M}(t, \omega)\right)^{2}\right]-\left(\mathbb{E}\left[y^{M}(t, \omega)\right]\right)^{2}=\int_{-\infty}^{\infty}\left(y^{M}(t)\right)^{2} f_{1}^{M}(y, t) d y-\left(\mu_{y^{M}}(t)\right)^{2}$,

where $\omega \in \Omega$. In Table 4.2, we show the values of errors defined in (4.22). These figures have been computed taking values of the truncation order $M$ so that error is less or equal than $10^{-2}$. Notice that greater values of $M$ are required to accomplish 

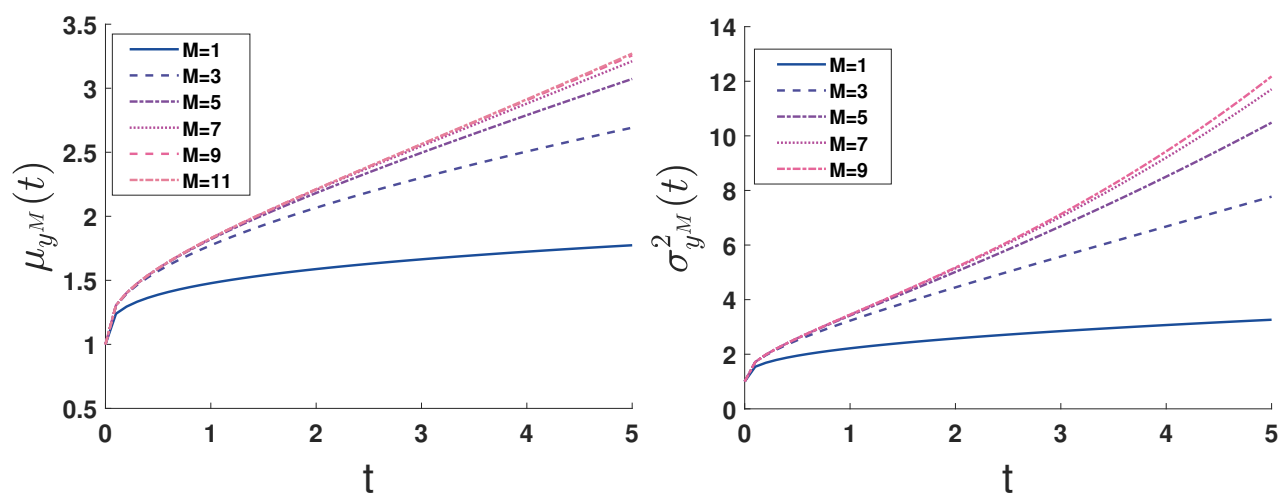

Figure 4.2: Plots of approximations $\mu_{y^{M}}(t)$ (left) and $\sigma_{y^{M}}^{2}(t)$ (right), given by (4.23) and (4.24), respectively, to the exact mean and variance of the solution SP to random fractional IVP (4.3), respectively, for different values of the truncation order $M$ in the context of Example 4.1.

for the stopping criterion of the error of the variance as expected. From this table we observe that both errors, $e_{M}^{\mu}$ and $e_{M}^{\sigma^{2}}$, decrease as $M$ increases.

Example 4.2 This second example aims to complete our numerical experiments by taking different probability distributions for random inputs and the order of fractional derivative from the ones chosen in Example 4.1. In this spirit, our subsequent presentation will be more concise since it follows in broad outline a similar structure as in Example 4.1. Let us consider the random fractional

\begin{tabular}{|c|c|c|c|c|c|c|c|}
\hline & $\mathrm{M}=1$ & $\mathrm{M}=2$ & $\mathrm{M}=3$ & $\mathrm{M}=4$ & $\mathrm{M}=5$ & $\mathrm{M}=6$ & $\mathrm{M}=7$ \\
\hline$e_{M}^{\mu}$ & 1.746 & 0.987 & 0.547 & 0.298 & 0.160 & 0.085 & 0.045 \\
\hline$\frac{e_{M}^{\sigma^{2}}}{\sigma^{2}}$ & 6.877 & 4.871 & 3.164 & 1.949 & 1.157 & 0.668 & 0.378 \\
\hline & $\mathrm{M}=8$ & $\mathrm{M}=9$ & $\mathrm{M}=10$ & $\mathrm{M}=11$ & & $=12$ & $\mathrm{M}=13$ \\
\hline$\overline{e_{M}^{\mu}}$ & 0.023 & 0.012 & 0.006 & - & & - & - \\
\hline$e_{M}^{\sigma^{2}}$ & 0.210 & 0.115 & 0.062 & 0.0335 & & 178 & 0.0094 \\
\hline
\end{tabular}

Table 4.2: Values of errors defined in (4.22) to quantify the quality of approximations $\mu_{y^{M}}(t)$ and $\sigma_{y^{M}}^{2}(t)$, given by $(4.23)$ and $(4.24)$, respectively, to the exact mean and variance of the solution SP to random fractional IVP (4.3), respectively, for different values of the truncation order $M$ in the context of Example 4.1. The truncation order $M$ has been computed so that $e_{M}(\hat{t}) \leq 10^{-2}$. 

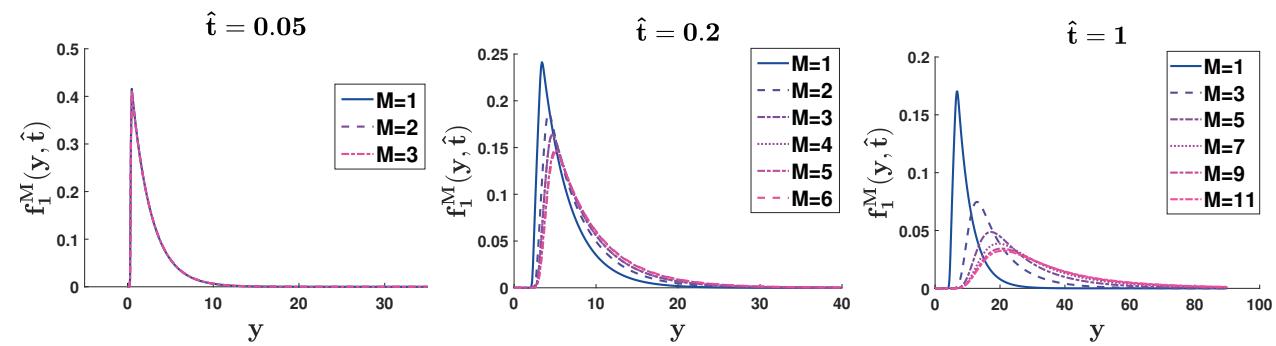

Figure 4.3: Plots of the 1-PDF, $f_{1}^{M}(y, t)$, given by (4.14), for different truncations $M$ at the time instants $\hat{t}=0.05$ (left), $\hat{t}=0.2$ (centre) and $\hat{t}=1$ (right) in Example 4.2.

\begin{tabular}{lcccccccccc}
\hline $\mathrm{e}_{M}(\hat{t})$ & $\mathrm{M}=1$ & $\mathrm{M}=2$ & $\mathrm{M}=3$ & $\mathrm{M}=4$ & $\mathrm{M}=5$ & $\mathrm{M}=6$ & $\mathrm{M}=7$ & $\mathrm{M}=8$ & $\mathrm{M}=9$ & $\mathrm{M}=10$ \\
\hline \hline$\hat{t}=0.05$ & 0.009 & - & - & - & - & - & - & - & - & - \\
\hline$\hat{t}=0.2$ & 0.338 & 0.159 & 0.077 & 0.038 & 0.018 & 0.009 & - & - & - & - \\
\hline$\hat{t}=1$ & 0.817 & 0.516 & 0.337 & 0.223 & 0.148 & 0.099 & 0.066 & 0.043 & 0.029 & 0.010 \\
\hline
\end{tabular}

Table 4.3: Values of error $e_{M}(\hat{t})$ defined in (4.21) at the time instants $\hat{t}=\{0.05,0.2,1\}$ in Example 4.2. The truncation order $M$ has been computed so that $e_{M}(\hat{t}) \leq 10^{-2}$.

IVP (4.3) where $\alpha=0.7, \lambda(\omega)$ is a Gaussian $R V$ with mean 1 and standard deviation $0.1, \lambda(\omega) \sim N(1 ; 0.1), \gamma(\omega)$ has a Uniform distribution on interval $[2,3]$, $\gamma(\omega) \sim U n(2,3)$ and $\beta_{0}(\omega)$ has a Gamma distribution with parameters 1 and 2 , $\beta_{0} \sim G a(1 ; 2)$. Hereinafter, we assume that $\lambda(\omega), \gamma(\omega)$ and $\beta_{0}(\omega)$ are independent, this ensures hypothesis $\boldsymbol{J 1}$ is fulfilled. Furthermore, it is clear that $\mathbb{E}[|\gamma|]=5 / 2$ and $\left|f_{\beta_{0}}^{\prime}\left(\beta_{0}\right)\right|=\left|\left(1 / 2 \mathrm{e}^{-\beta_{0} / 2}\right)^{\prime}\right| \leq 1 / 4$ for all $\beta_{0}>0$. As a consequence, hypotheses J2 and J3 hold.

In Figure 4.3, we have plotted the 1-PDF, $f_{1}^{M}(y, t)$ given in (4.14), for different orders of truncation $M$ and time instants $\hat{t}=\{0.05,0.2,1\}$. From these graphical representations, we can observe the convergence of approximations even using few terms. As a measure of the accuracy of these approximations, in Table 4.3 we show the values of error defined in (4.21) time instants $\hat{t}=\{0.05,0.2,1\}$ taking as stopping criterion for the order of truncation $M$ that $e_{M}(\hat{t}) \leq 10^{-2}$.

Finally, in Figure 4.4 the mean, $\mu_{y^{M}}(t)$ and the variance $\sigma_{y^{M}}^{2}(t)$ for different truncation order $M$ are plotted to $t$ from 0 to 1 . As in the case of the 1-PDF we can observe the convergence. In Table 4.4 the following error for the mean and the variance is calculated as in Example 4.2. 

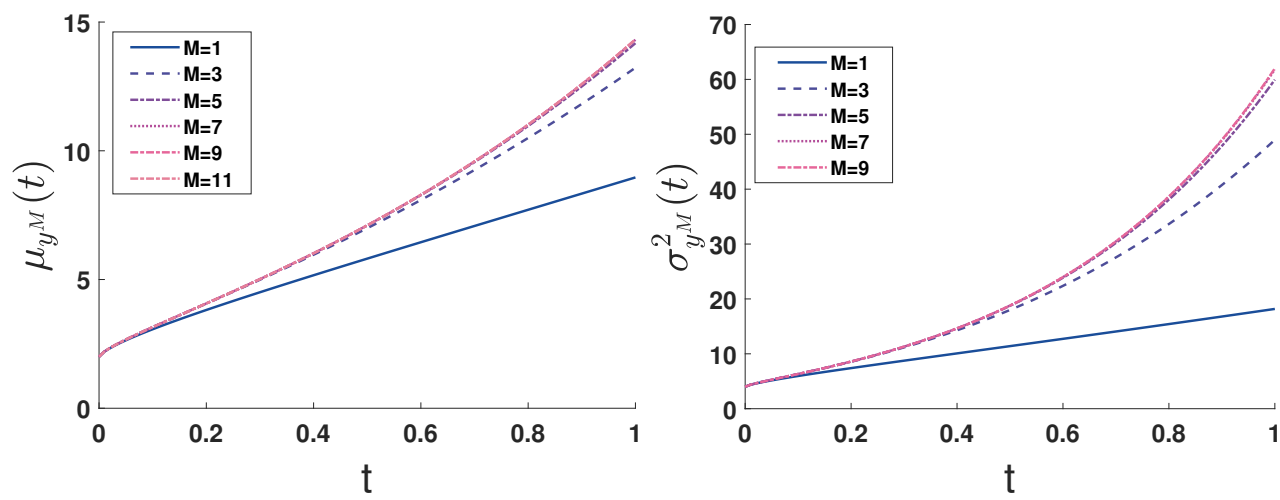

Figure 4.4: Plots of approximations $\mu_{y^{M}}(t)$ (left) and $\sigma_{y^{M}}^{2}(t)$ (right), given by (4.23) and (4.24), respectively, to the exact mean and variance of the solution SP to random fractional IVP (4.3), respectively, for different values of the truncation order $M$ in the context of Example 4.2 .

\begin{tabular}{ccccccc}
\hline & $\mathrm{M}=1$ & $\mathrm{M}=2$ & $\mathrm{M}=3$ & $\mathrm{M}=4$ & $\mathrm{M}=5$ & $\mathrm{M}=6$ \\
\hline \hline$e_{M}^{\mu}$ & 1.062 & 0.454 & 0.173 & 0.060 & 0.019 & 0.006 \\
\hline$e_{M}^{\sigma^{2}}$ & 0.712 & 0.331 & 0.136 & 0.050 & 0.017 & 0.005 \\
\hline
\end{tabular}

Table 4.4: Values of errors defined in (4.22) to quantify the quality of approximations $\mu_{y^{M}}(t)$ and $\sigma_{y^{M}}^{2}(t)$, given by $(4.23)$ and $(4.24)$, respectively, to the exact mean and variance of the solution SP to random fractional IVP (4.3), respectively, for different values of the truncation order $M$ in the context of Example 4.2. The truncation order $M$ has been computed so that $e_{M}(\hat{t}) \leq 10^{-2}$. 


\subsection{Conclusions}

In this chapter, we have computed approximations to the first probability density function of random fractional linear differential equations using the so-called Random Variable Transformation method. Under mild conditions on random data, we have proved the convergence of approximations to the exact probability density function of the solution SP. The generality of our findings relies upon the facts that uncertainty has been considered in all inputs (initial condition, forcing term and diffusion coefficient) and a wide variety of probability distributions can be assigned to each one of them. To the best of our knowledge, this is the first time this analysis has been conducted for RFDEs.

\section{Chapter published}

The results of this chapter have been published in [20]. With regard to this paper, the $\mathrm{PhD}$ candidate has contributed by working in its complete development with more emphasis on preparing the numerical examples and writing the results submitted to a scientific journal. 



\section{Chapter}

\section{Solving a random mean square}

non-autonomous linear fractional differential equations by means of a generalized mean square convergent power series

In the previous chapters a random linear fractional differential equation has been studied. A convergent series solution and its first probability density function have been obtained. In this chapter, a random non-autonomous linear fractional differential equation of order $\alpha \in] 0,1]$ is solved. A mean square series solution is constructed using a random Frobenius approach. Its mean square convergence is guaranteed assuming mild conditions on its random inputs. In addition, explicit expressions to approximate the mean, the variance and the covariance of the random series solution are given. Two full illustrative examples are shown. In the second of them, the applicability of random fractional calculus in mathematical modelling is shown describing the growth dynamics of a bacteria over the time using real data. 


\subsection{Introduction}

This chapter is devoted to solve, the following random non-autonomous fractional IVP using a generalized version of Frobenius method (see Section 2.4).

$$
\left\{\begin{array}{cl}
\left({ }^{C} D_{0^{+}}^{\alpha}\right)(t)-B t^{\beta} Y(t) & =0, \quad t>0, \quad 0<\alpha \leq 1, \quad \beta>0 \\
Y(0) & =A
\end{array}\right.
$$

where $\left({ }^{C} D_{0^{+}}^{\alpha} Y\right)(t)$, is the random m.s. Caputo fractional derivative defined in (2.16). The input data $A$ and $B$ are assumed to be independent 2-RVs.

The aim of the chapter is to find general conditions on 2-RVs $A, B$ so that for the random IVP (5.1) we can construct a m.s. solution of the form

$$
Y(t)=\sum_{m=0}^{\infty} X_{m} t^{(\alpha+\beta) m}
$$

where $\left\{X_{m}: m \geq 0\right\}$ is a sequence of $2-R V$ s to be determined. The study will be conducted by using the random m.s. calculus, see Section 2.2 and [109].

This chapter is organized as follows. Section 5.2, is devoted to construct a m.s. convergent solution of IVP (5.1) and to establish mild conditions into the random inputs to guarantee the convergence of the generalized power series solution. Furthermore, the main statistical properties, mean, variance and covariance of the solution SP are obtained. In Section 5.3 two numerical examples are described to illustrate the theoretical findings.

\subsection{Constructing a mean square convergent random generalized power series solution and approximating its main statistical properties}

First, we shall justify that the first m.s. derivative of the 2-SP $Y(u)$ defined in (5.2) at $t=u>0$ is given by

$$
Y^{\prime}(u)=\sum_{m=0}^{\infty} X_{m}(\alpha+\beta) m u^{(\alpha+\beta) m-1}
$$


To this end, we apply Theorem 2.1 and Proposition 2.4. Let $u_{0}>0$ be fixed and define $V_{m}(u)=X_{m} u^{(\alpha+\beta) m}$. Let us assume that $X_{m}$ is a 2-RV. By applying Theorem. 2.1, with $f(u)=u^{(\alpha+\beta) m}$ and $Z(u)=X_{m}$, it follows that for each $m, V_{m}(u)$ is m.s differentiable at $u=u_{0}$ and its m.s. derivative is given by $V_{m}^{\prime}\left(u_{0}\right)=X_{m}(\alpha+\beta) m u_{0}^{(\alpha+\beta) m-1}$. It is easy to check that $V_{m}(u)$ is m.s. continuous at $u_{0}$. Once coefficients $X_{m}$ are determined, we will find conditions on RVs $A, B$ in order to hypotheses ii) and iii) of Proposition 2.4 are met. For now on, assume that $I \subset[0, \infty)$. If $V(u)=\sum_{n \geq m_{0}} V_{n}(u)$ is m.s. convergent on $I$ and $\sum_{n \geq m_{0}} V_{m}^{\prime}(u)$ is m.s. uniformly (m.s.u.) convergent in a neighbourhood of each $u \in I$, then Proposition 2.4 implies (5.3) and

$$
\begin{aligned}
\left({ }^{C} D_{0^{+}}^{\alpha} Y\right)(t) & =\frac{1}{\Gamma(1-\alpha)} \int_{0}^{t}(t-u)^{-\alpha} Y^{\prime}(u) \mathrm{d} u \\
& =\frac{1}{\Gamma(1-\alpha)} \int_{0}^{t}(t-u)^{-\alpha} \sum_{m=0}^{\infty} X_{m}(\alpha+\beta) m u^{(\alpha+\beta) m-1} \mathrm{~d} u \\
& =\sum_{m=0}^{\infty} X_{m}(\alpha+\beta) m \frac{1}{\Gamma(1-\alpha)} \int_{0}^{t}(t-u)^{-\alpha} u^{(\alpha+\beta) m-1} \mathrm{~d} u .
\end{aligned}
$$

The commutation of the series in the last step is legitimated because it is m.s.u. convergent. Now, using the substitution $u=v t$ on the above integral and the relationship (2.10) one gets

$$
\begin{aligned}
\frac{1}{\Gamma(1-\alpha)} \int_{0}^{t}(t-u)^{-\alpha} u^{(\alpha+\beta) m-1} \mathrm{~d} u & =\frac{t^{m(\beta+\alpha)-\alpha}}{\Gamma(1-\alpha)} \int_{0}^{1}(1-v)^{-\alpha} v^{m(\alpha+\beta)-1} \mathrm{~d} v \\
& =\frac{\Gamma(m(\alpha+\beta))}{\Gamma(m(\alpha+\beta)-\alpha+1)} t^{m(\beta+\alpha)-\alpha} .
\end{aligned}
$$

Therefore,

$$
\begin{aligned}
\left({ }^{C} D_{0^{+}}^{\alpha} Y\right)(t) & =\sum_{m=0}^{\infty} X_{m}(\alpha+\beta) m \frac{\Gamma(m(\alpha+\beta))}{\Gamma(m(\alpha+\beta)-\alpha+1)} t^{m(\beta+\alpha)-\alpha} \\
& =\sum_{m=0}^{\infty} X_{m+1} \frac{\Gamma((m+1)(\alpha+\beta)+1)}{\Gamma((m+1)(\alpha+\beta)-\alpha+1)} t^{(m+1)(\beta+\alpha)-\alpha},
\end{aligned}
$$


in the last step we have used the gamma duplication formula, $x \Gamma(x)=\Gamma(x+1)$, $x>0$ with $x=(m+1)(\alpha+\beta)>0$. Substituting (5.6) into (5.1) yields

$$
\begin{aligned}
& \left({ }^{C} D_{0^{+}}^{\alpha} Y\right)(t)-B t^{\beta} Y(t) \\
& =\sum_{m=0}^{\infty}\left[X_{m+1} \frac{\Gamma((m+1)(\alpha+\beta)+1)}{\Gamma((m+1)(\alpha+\beta)-\alpha+1)}-B X_{m}\right] t^{(m+1)(\beta+\alpha)-\alpha}=0 .
\end{aligned}
$$

If $X_{m+1} \frac{\Gamma((m+1)(\alpha+\beta)+1)}{\Gamma((m+1)(\alpha+\beta)-\alpha+1)}=B X_{m}, m=0,1,2, \ldots$ As $Y(0)=A=X_{0}$, it follows from last equation that $X_{m}=B^{m} A \prod_{n=1}^{m} \frac{\Gamma((n-1) \alpha+\beta n+1)}{\Gamma(n(\alpha+\beta)+1)}$ for $m \geq 1$. As a result,

$$
Y(t)=A+\sum_{m=1}^{\infty} B^{m} A \prod_{n=1}^{m} \frac{\Gamma((n-1) \alpha+\beta n+1)}{\Gamma(n(\alpha+\beta)+1)} t^{(\alpha+\beta) m}
$$

is a m.s. solution of random IVP (5.1) on $I$ provided that $Y(t)$ is m.s. convergent on $I$ and $Y^{\prime}(t)=\sum_{m=1}^{\infty} B^{m} A(\alpha+\beta) m \prod_{n=1}^{m} \frac{\Gamma((n-1) \alpha+\beta n+1)}{\Gamma(n(\alpha+\beta)+1)} t^{(\alpha+\beta) m-1}$ is m.s.u. convergent on $I$. Let us first show that $Y(t)$ is m.s. convergent on $I$. To this end, we will assume the following hypotheses

M1: For $m, m_{0}$ integers

$$
\exists \eta, \mathcal{H}>0, p \geq 0:\left\|B^{m}\right\|_{2} \leq \eta \mathcal{H}^{m-1}((m-1) !)^{p}, \quad \forall m: m \geq m_{0} \geq 1 .
$$

This condition have been previously described in Chapter 3, hypothesis $\hat{\mathbf{H}} \mathbf{2}$, (page 49), but considering the RV $\lambda$ instead $B$.

M2: $A$ and $B$ are independent RVs.

Now, 


$$
\begin{gathered}
\left\|B^{m} A \prod_{n=1}^{m} \frac{\Gamma((n-1) \alpha+\beta n+1)}{\Gamma(n(\alpha+\beta)+1)} t^{(\alpha+\beta) m}\right\|_{2} \leq \eta \mathcal{H}^{m-1}((m-1) !)^{p}\|A\|_{2} \\
\cdot \prod_{n=1}^{m} \frac{\Gamma((n-1) \alpha+\beta n+1)}{\Gamma(n(\alpha+\beta)+1)} t^{(\alpha+\beta) m} \\
:=\delta_{m}(t) .
\end{gathered}
$$

The analysis of the convergence of the series $\sum_{m=0}^{\infty} \delta_{m}(t)$ will be performed by using the ratio or D'Alembert test. Indeed, we compute the $\lim _{m \rightarrow \infty} \frac{\delta_{m+1}(t)}{\delta_{m}(t)}$ with the aid of Stirling's formula, (2.12):

$$
\begin{aligned}
& \lim _{m \rightarrow \infty} \frac{\delta_{m+1}(t)}{\delta_{m}(t)}=\lim _{m \rightarrow \infty} \mathcal{H} m^{p} \frac{\Gamma(m \alpha+(m+1) \beta+1)}{\Gamma((m+1)(\alpha+\beta)+1)} t^{\alpha+\beta} \\
& =\lim _{m \rightarrow \infty} \frac{\mathcal{H} m^{p}(m \alpha+(m+1) \beta)^{m \alpha+(m+1) \beta} e^{-(m \alpha+(m+1) \beta)} \sqrt{2 \pi(m \alpha+(m+1) \beta)}}{[(m+1)(\alpha+\beta)]^{(m+1)(\alpha+\beta)} e^{-(m+1)(\alpha+\beta)} \sqrt{2 \pi(m+1)(\alpha+\beta)}} t^{\alpha+\beta} \\
& =\lim _{m \rightarrow \infty} \mathcal{H} m^{p}\left[\frac{m \alpha+(m+1) \beta}{(m+1) \beta+(m+1) \alpha}\right]^{m(\alpha+\beta)}\left[\frac{m \alpha+(m+1) \beta}{(m+1) \beta+(m+1) \alpha}\right]^{\beta} \\
& \times\left(\frac{1}{\beta+\alpha}\right)^{\alpha}\left(\frac{1}{m+1}\right)^{\alpha} e^{\alpha} \sqrt{\frac{m \alpha+(m+1) \beta}{(m+1) \beta+(m+1) \alpha}} t^{\alpha+\beta} .
\end{aligned}
$$

As $\lim _{m \rightarrow+\infty}\left[\frac{m \alpha+(m+1) \beta}{(m+1) \beta+(m+1) \alpha}\right]^{m}=e^{-\frac{\alpha}{\alpha+\beta}}, \lim _{m \rightarrow+\infty} \frac{m \alpha+(m+1) \beta}{(m+1) \beta+(m+1) \alpha}=1$, for $0 \leq$ $p \leq \alpha$ and $t \geq 0$ it follows

$$
\begin{aligned}
\lim _{m \rightarrow+\infty} \frac{\delta_{m+1}(t)}{\delta_{m}(t)} & =t^{\alpha+\beta} \frac{\mathcal{H}}{(\alpha+\beta)^{\alpha}} \lim _{m \rightarrow+\infty} \frac{m^{p}}{(m+1)^{\alpha}} \\
& =\mathcal{H} t^{\beta}\left(\frac{t}{\alpha+\beta}\right)^{\alpha} \lim _{m \rightarrow+\infty} \frac{m^{p}}{(m+1)^{\alpha}} \\
& = \begin{cases}0 & \text { if } 0 \leq p<\alpha, \\
\mathcal{H} t^{\beta}\left(\frac{t}{\alpha+\beta}\right)^{\alpha} & \text { if } p=\alpha .\end{cases}
\end{aligned}
$$


Therefore, the series $\sum_{m=0}^{\infty} \delta_{m}(t)$ is convergent for all $t$ in $\mathbb{D}$, where

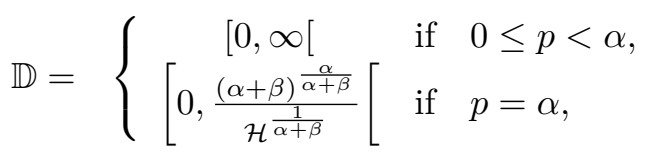

which implies that $Y(t)$ is m.s. convergent for every $t$ in $\mathbb{D}$. Following a similar analysis, it can be shown that $Y^{\prime}(t)$ is m.s. convergent for every $t$ in $\mathbb{D}$. We conclude that for any closed interval $I$ in $\mathbb{D}, Y(t)$ is m.s convergent on $I$ and $Y^{\prime}(t)$ m.s.u. convergent on $I$. As a consequence, hypotheses i) and ii) of Proposition 2.4 hold. Hence, we have established the following result:

Theorem 5.1 If the RVs A,B satisfy conditions $\mathbf{M 1}$ and $\mathbf{M 2}$, then the 2-SP $Y(t)$ defined by (5.8) is a m.s. solution of the random IVP (5.1) on any closed interval $I \subset \mathbb{D}$, where $\mathbb{D}$ is defined in (5.12).

As the m.s. solution $Y(t)$ of random IVP (5.1) is a 2-SP represented through an infinite series (see expression (5.8)), in practice, must be truncated at a positive integer $M$,

$$
Y_{M}(t)=A+\sum_{m=1}^{M} B^{m} A G_{m} t^{(\alpha+\beta) m}, \quad G_{m}:=\prod_{n=1}^{m} \frac{\Gamma((n-1) \alpha+\beta n+1)}{\Gamma(n(\alpha+\beta)+1)}
$$

The main statistical information of $Y(t)$ is given by the mean, the variance and the covariance functions. As $Y(t)$ is convergent in the m.s. sense, Proposition 2.10 legitimates the mean, variance and covariance of $Y(t)$ can be computed obtaining the main statistical moments of $Y_{M}(t)$.Considering that $A$ and $B$ are independent RVs, the mean of $Y_{M}(t)$ can be written as

$$
\mathbb{E}\left[Y_{M}(t)\right]=\mathbb{E}[A]+\sum_{m=1}^{M} \mathbb{E}\left[B^{m}\right] \mathbb{E}[A] G_{m} t^{(\alpha+\beta) m}
$$

The covariance of two any $\operatorname{RVs} \tilde{A}$ and $\tilde{B}$ is defined by $\operatorname{Cov}[\tilde{A}, \tilde{B}]=\mathbb{E}[\tilde{A} \tilde{B}]-$ $\mathbb{E}[\tilde{A}] \mathbb{E}[\tilde{B}]$, and in particular, $\operatorname{Cov}[\tilde{A}, \tilde{A}]=\mathbb{E}\left[\tilde{A}^{2}\right]-(\mathbb{E}[\tilde{A}])^{2}=\mathbb{V}[\tilde{A}]$, where $\mathbb{V}[\tilde{A}]$ 
denotes the variance of $\tilde{A}$, the covariance of $Y_{M}(t)$ and $Y_{N}(s)$ with $M, N$ positive integers and $t, s$ in $I \subset \mathbb{R}$ is given by

$$
\begin{aligned}
\operatorname{Cov}\left[Y_{M}(t), Y_{N}(s)\right]= & \operatorname{Cov}\left[A+\sum_{m=1}^{M} B^{m} A G_{m} t^{(\alpha+\beta) m}, A+\sum_{n=1}^{N} B^{n} A G_{n} s^{(\alpha+\beta) n}\right] \\
& +\sum_{n=1}^{N} \sum_{m=1}^{M} \operatorname{Cov}\left[B^{m} A, B^{n} A\right] G_{m} G_{n} t^{(\alpha+\beta) m} s^{(\alpha+\beta) n} \\
= & \mathbb{V}[A]\left(1+\sum_{n=1}^{N} \mathbb{E}\left[B^{n}\right] G_{n} t^{(\alpha+\beta) m}+\sum_{m=1}^{M} \mathbb{E}\left[B^{m}\right] G_{m} t^{(\alpha+\beta) m}\right) \\
& +\sum_{n=1}^{N} \sum_{m=1}^{M}\left(\mathbb{E}\left[A^{2}\right] \mathbb{E}\left[B^{m+n}\right]-(\mathbb{E}[A])^{2} \mathbb{E}\left[B^{m}\right] \mathbb{E}\left[B^{n}\right]\right) \\
& \cdot G_{m} G_{n} t^{(\alpha+\beta) m} s^{(\alpha+\beta) n} .
\end{aligned}
$$

Since $\mathbb{V}\left[Y_{M}(t)\right]=\mathbb{C o v}\left[Y_{M}(t), Y_{M}(t)\right]$, setting $M=N$ and $t=s$ in (5.15), one also obtains the variance of $Y_{M}(t)$

$$
\begin{aligned}
\mathbb{V}\left[Y_{M}(t)\right]= & \mathbb{V}[A]\left(1+2 \sum_{m=1}^{M} \mathbb{E}\left[B^{m}\right] G_{m} t^{(\alpha+\beta) m}\right) \\
& +\sum_{n=1}^{M} \sum_{m=1}^{M}\left(\mathbb{E}\left[A^{2}\right] \mathbb{E}\left[B^{m+n}\right]-(\mathbb{E}[A])^{2} \mathbb{E}\left[B^{m}\right] \mathbb{E}\left[B^{n}\right]\right) \\
& \cdot G_{m} G_{n} t^{(\alpha+\beta)(m+n)} .
\end{aligned}
$$

\subsection{Numerical examples}

Now, we first illustrate the theoretical results previously established through two examples. The first one is a full numerical example while the second example illustrates the potentiality of random fractional IVP (5.1) in a mathematical modelling setting using real data. 
Example 5.1 This example has been devised to illustrate the different domains of convergence for the mean and standard deviation depending upon the relationship between parameters $p$ and $\alpha$ (see Theorem 5.1 and expression (5.12)). Let us consider the random fractional IVP (5.1) in two scenarios (Cases I and II) depending on the order $\alpha \in] 0,1]$ of the fractional derivative, the parameter $\beta$ and the probability distributions chosen for $R V s B$ and $A$.

Case $I(p<\alpha): \alpha=0.7, B$ is a Beta $R V$ of parameters $(50,100)$, i.e., $B \sim$ $B e(50 ; 100)$ (thus, according to Example 3.1, $p=0$ because $B$ is a bounded $R V)$; $A$ is a Gaussian $R V$ with mean $\mu=0.1$ and variance $\sigma^{2}=1$, i.e., $A \sim N(0.1 ; 1)$ and, $\beta=0.1$. In Figure 5.1, we have plotted approximations of the mean and standard deviation by expressions (5.14) and (5.16), respectively, using different orders of truncations $M$ over the interval $t \in[0,15]$. Notice that these results are in agreement with our theoretical findings. Indeed, as $p=0<0.7=\alpha$, we can observe that both statistical moments converge for every value of $t$.

Case II $(p=\alpha): \alpha=0.5, B \sim N(0 ; 0.1)$ (thus, $p=0.5), A \sim N(0.1 ; 1)$ and, $\beta=2$. As $p=\alpha$, according to Theorem 5.1 and expression (5.12), the domain of convergence is $\mathbb{D}=[0,2.626578[$ since $\eta=\sigma=0.1$ and $\mathcal{H}=\sigma \sqrt{2} \approx 0.141421$ (see Example 3.2). In Figure 5.2 , we have plotted approximations of the mean and standard deviation using different orders of truncations $M$ over the time intervals $t \in[0,3.5]$ and $t \in[0,3]$, respectively. To delineate the region of convergence we have plotted a vertical red line. For the sake of clarity, a part of the region of convergence has been magnified for both the mean and the standard deviation (right column of Case II in Figure 5.2). The numerical results agree with theoretical findings.

When $p=\alpha$, as reported in (5.12), the domain of convergence $\left[0, t_{1}\right.$ [ of the solution SP may be small (it will depend on the fractional derivative order $\alpha, \beta$ model parameter and the constant $\mathcal{H}$ that appears in hypothesis $\mathbf{M 1})$. This domain $\left[0, t_{1}[\right.$ 

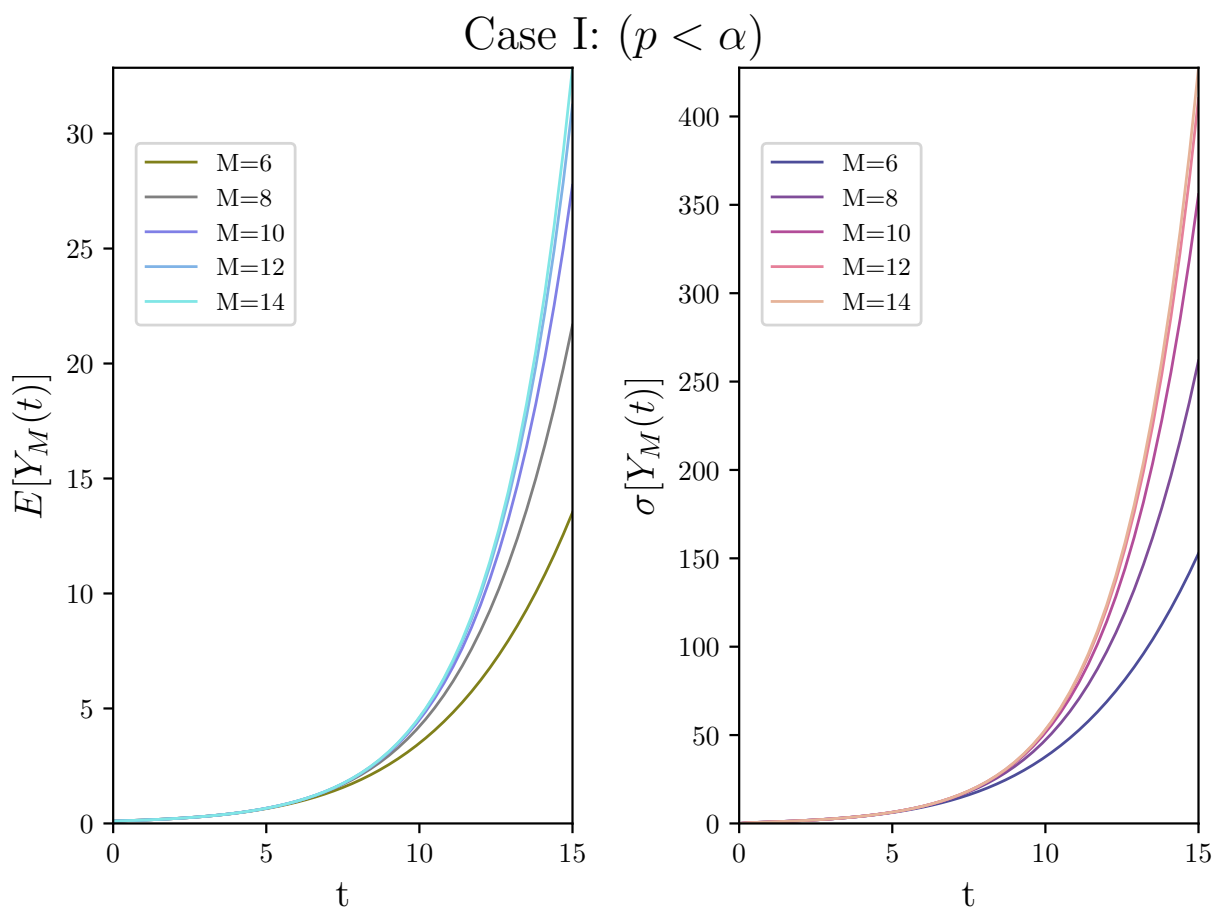

Figure 5.1: Approximations of the mean and the standard deviation of the solution SP to the random IVP (5.1) using different orders of truncations $M$ in Case I $(p<\alpha)$ described in the context of Example 6.1. Notice that the approximations corresponding to $M=12$ and $M=14$, for the mean and the standard deviation, match on the whole time interval $t \in[0,15]$, thus showing convergence. 
Case II: $(p=\alpha)$
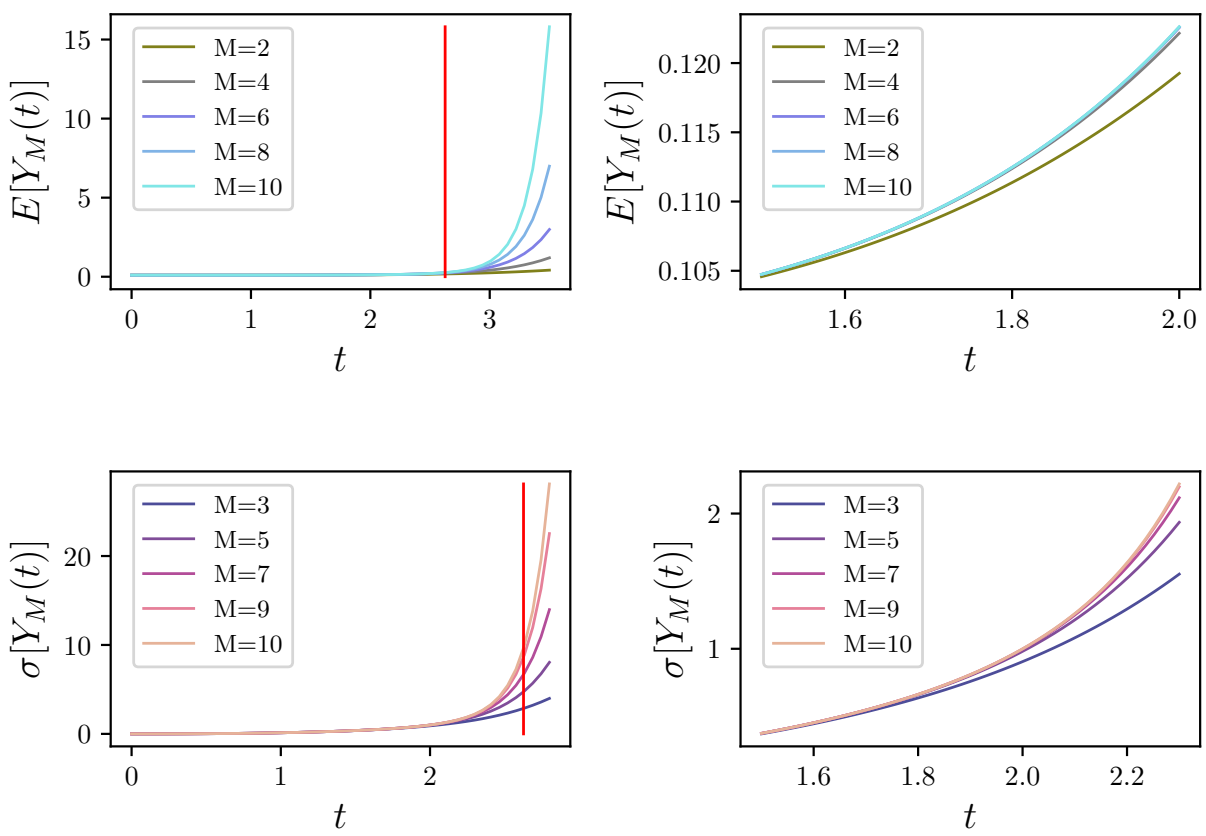

Figure 5.2: Approximations of the mean and the standard deviation of the solution SP to the random IVP (5.1) using different orders of truncations $M$ in Case II $(p=\alpha)$ described in the context of Example 6.1. On the left side, we have delineated the domain of convergence for the mean and the standard deviation plotting a vertical line. On the right side, we show a zoom on a piece of the domain of convergence, for the sake of clarity. Observe that the approximations corresponding to $M=4,6,8,10$ match for the mean, while this same fact happens when $M=9,10$, in the case of the standard deviation. 
with $t_{1}=\left((\alpha+\beta)^{\frac{\alpha}{\alpha+\beta}}\right) /\left(\mathcal{H}^{\frac{1}{\alpha+\beta}}\right)$, can be extended using the following strategy, which has been successfully applied in another contributions, [73]. Once the solution $Y(t)$, given by (5.8), has been constructed in the interval $\left[0, t_{1}[\right.$, we seek a solution $\mathrm{SP}$, say $Y_{1}(t)$, of the form

$$
Y_{1}(t)=\sum_{m=0}^{\infty} X_{m, 1}\left(t-t_{1}\right)^{(\alpha+\beta) m}
$$

i.e., centered at $t_{1}$, of the same RFDE given in (5.1), but whose random initial condition matches the value of the solution, $Y(t)$, constructed in the piece $\left[0, t_{1}[\right.$ at the ending time point, that is, $Y_{1}\left(t_{1}\right):=Y\left(t_{1}\right)$. Then, using a similar reasoning we have exhibited in our development, one can determine the new coefficient RVs $X_{m, 1}, m \geq 0$, and it can be proven that random series (5.17) is m.s. convergent in the piece $\left[t_{1}, 2 t_{1}[\right.$. This procedure can be successively applied to extend the solution on a desired interval, say $[0, T]$.

Example 5.2 Now, we illustrate an application of random fractional IVP (5.1) to model the dynamics of growth bacteria over the time using real data. Differential equation in (5.1) can be interpreted as a generalization of the classical exponential (or Malthusian) model with time-dependent population growth rate, $B t^{\beta}$, for a species whose initial population, A is known. Here, this generalization has been made in two senses, namely, first introducing the Caputo fractional derivative, $\left({ }^{C} D_{0^{+}}^{\alpha} Y\right)(t)$ with $0<\alpha \leq 1$, instead of classical derivative, $Y^{\prime}(t)$, and secondly, by considering model parameter $B$ and initial condition $A$ as $R V$ s rather than deterministic values. On the one hand, the use of a fractional derivative can be justified because the growth dynamics is determined by genetic, environmental factors, etc., developed over previous periods, then it is expected these biological features can be better modelled via Caputo fractional derivative, which is defined in terms of an integral (thus with memory), instead of classical derivative that just characterizes instantaneous changes. On the other hand, the consideration of randomness in model inputs $B$ and $A$ can be justified because the complex nature of population growth rate, which depends on uncertain biological factors, and, in practice, the value of the initial condition is usually known on the basis of sampling, respectively. In this spirit, here we consider the classical non-autonomous Malthus model to a generalized one, in which ordinary derivative and model inputs are 
replaced by fractional derivatives and $R V s$, respectively. Our example is based on measured population values of Rhodobacter Capsulatus anaerobic photosynthetic bacteria $\left\{y_{i}: 0 \leq i \leq 4\right\}$ corresponding to days $t_{i}$ (Table 5.1). This information has been obtained from source [110]. First, we have performed a classical (or deterministic) fitting based upon minimizing the m.s. error between real data $y_{i}$, and the solution of the corresponding deterministic differential equation $\left(Y^{\prime}(t)=\right.$ $\left.B t^{\beta} Y(t), Y(0)=A=5.83 \times 10^{5}\right)$, which is given by

$$
Y(t)=A \exp \left(\frac{B}{1+\beta}+\frac{B t^{1+\beta}}{1+\beta}\right) .
$$

Using PSO (Particle Swarm Optimization method) with 1000 iterations [41], we have obtained the following estimates for deterministic model parameters: $\beta=2.2573$ and $B=0.168764$, being the RMSE (Root Mean Square Error) of this fitting $\epsilon^{\text {det. }}=763$ (observe that units are of magnitude $10^{6}$. In Table 5.1 we show the results, $y_{i}^{\text {det. }}$, provided by this approach. Secondly, we have assumed that $B$ is a Gamma $R V$ of parameters $\left(r_{B}, s_{B}\right)$ and the initial condition $A$ is an Exponential $R V$ of parameter $\lambda_{A}=1 /\left(5.83 \times 10^{5}\right)$. On the one hand, observe that the choice made for the distribution of $B$ is justified because real data $y_{i}$ have a positive trend, hence $B$ must be a positive $R V$ and Gamma distribution holds this feature, moreover it is a flexible distribution able to perform a good fitting since it depends on two parameters $\left(r_{B}\right.$ and $\left.s_{B}\right)$. On the other hand, Exponential distribution guarantees the positiveness of the initial condition, and we have imposed that its mean $\mathbb{E}[A]=1 / \lambda_{A}$ matches the initial condition $5.83 \times 10^{5}$. Then, considering this choice for the distributions of random inputs $B$ and $A$, we have performed a (random) fitting based upon minimizing the m.s. error between real data $y_{i}$ and the mean of the solution SP of the random fractional IVP (5.1), i.e.,

$$
\min _{r_{B}, s_{B}, \beta>0 ; 0<\alpha \leq 1} \operatorname{Error}\left(r_{B}, s_{B}, \beta, \alpha\right)=\sum_{i=0}^{4}\left(y_{i}-\mathbb{E}\left[Y_{M}\left(t_{i} ; r_{B}, s_{B}, \beta, \alpha\right)\right]\right)^{2},
$$

where $\mathbb{E}\left[Y_{M}\left(t_{i} ; r_{B}, s_{B}, \beta, \alpha\right)\right]$ is given by (5.13)-(5.14). We have again applied PSO method with 1000 iterations to solve this minimization program taking as truncation order $M=20$ (for which the approximation of the exact expectation is very accurate) and then we have obtained the following estimates for model 
parameters: $\beta=0.1975, r_{B}=14.64, s_{B}=75.32$ and $\alpha=0.89$, being the $R M S E$ (Root Mean Square Error) of this fitting $\epsilon^{\text {random }}=660$. In Table 5.1 we show the results, $y_{i}^{\text {random }}$, provided by this approach. We have shown that $\epsilon^{\text {random }}<\epsilon^{\text {det. }}$, in order to complete better an adequate comparison between deterministic and random fractional approaches, in Table 5.1 we give an important goodness-of-fit measure, MAPE (Mean Absolute Percentage Error). Again, we can observe that our proposed approach provides better results for this statistical measure.

\begin{tabular}{|c|c|c|c|c|c|c|}
\hline$t_{i}$ (time in days) & 0 & 2 & 4 & 7 & 9 \\
\hline$y_{i}$ (population cells $\left./ \mathrm{mL}\right)$ & $5.830 \mathrm{E}+05$ & $6.350 \mathrm{E}+05$ & $1.08 \mathrm{E}+06$ & $3.20 \mathrm{E}+06$ & $5.23 \mathrm{E}+06$ & -- \\
\hline$y_{i}^{\text {det. }}$ (deterministic fitting) & $6.667 \mathrm{E}+05$ & $9.189 \mathrm{E}+05$ & $1.435 \mathrm{E}+06$ & $3.141 \mathrm{E}+06$ & $5.589 \mathrm{E}+06$ & 0.20144 \\
\hline$y_{i}^{\text {random }}$ (random fitting) & $5.830 \mathrm{E}+05$ & $8.504 \mathrm{E}+05$ & $1.338 \mathrm{E}+06$ & $2.932 \mathrm{E}+06$ & $5.307 \mathrm{E}+06$ & 0.13533 \\
\hline
\end{tabular}

Table 5.1: Cell counts $y_{i}$ of Rhodobacter Capsulatus anaerobic photosynthetic bacteria at the time instants $t_{i}$ (data retrieved from [110]). Values of the deterministic fitting ( $y_{i}^{\text {det. }}$ ) and random fractional fitting $y_{i}^{\text {random }}$. Goodness-of-fit measure for both approaches: MAPE (Mean Absolute Percentage Error). Example 5.2.

\subsection{Conclusions}

In this chapter, we have studied a random non-autonomous linear fractional differential equation, where the order of the derivative $\alpha \in] 0,1]$. A generalized power series solution has been constructed using a randomized version of the classical Frobenius method, and mild conditions have been imposed to guarantee its m.s. convergence. Afterwards, we have computed approximations for the mean, for the variance and for the covariance of the solution SP. To illustrate the theoretical findings, two numerical examples have been shown. In particular, in the second one, we can observe the applicability of random fractional calculus in modelling of biological processes. We want to underline that this study seeks to contribute to the emergent area of random fractional differential equations (RFDEs), where fractional calculus and differential equations meet to provide a rigorous treatment of randomness. 


\section{Chapter published}

The results of this chapter have been published in [21]. With regard to this paper, the $\mathrm{PhD}$ candidate has contributed by working in its complete development with more emphasis on constructing a mean square convergent solution, obtaining their main statistical moments and developing numerical examples. 


\section{Chapter}

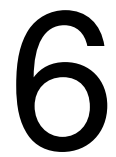

\section{Solving high order random mean square}

non-autonomous linear fractional differential equations: A generalized version of Airy random differential equation

In Chapter 5 a random non-autonomous linear fractional differential equation where the order of Caputo derivative, $\alpha$, lies in [0,1] has been studied. In this chapter, we advance solving the same non-autonomous IVP, but considering an arbitrary order of the fractional derivative. This IVP generalizes the Airy differential equation, since when $\alpha=2$ and $\beta=1$ it becomes the classical Airy IVP. As it has been done in the previous chapters, under appropriate hypotheses assumed upon the input data, we construct a convergent random generalized power series solution of the problem. Afterwards, we provide reliable explicit approximations for the main statistical information of the solution process (mean, variance and covariance). Finally, we illustrate how to construct reliable approximations of the probability density function of the solution stochastic process combining the approximations of the mean and variance with the Principle of Maximum Entropy. The theoretical findings are illustrated with numerical results. 


\subsection{Introduction}

Let us consider the following random non-autonomous linear fractional differential equation that generalizes the classical Airy-type IVP.

$$
\left\{\begin{aligned}
\left({ }^{C} D_{0^{+}}^{\alpha} Y\right)(t)-B t^{\beta} Y(t) & =0, \quad t>0, n-1<\alpha \leq n, \quad n \in \mathbb{N}, \quad \beta>0, \\
Y^{(j)}(0) & =A_{j}, \quad j=0,1, \ldots, n-1,
\end{aligned}\right.
$$

where $\left({ }^{C} D_{0^{+}}^{\alpha} Y\right)(t)$ is the m.s. random Caputo fractional derivative defined in (2.16). Notice that if $\alpha=2$ and $\beta=1$, then (6.1) becomes the classical Airy differential equation with initial conditions $A_{0}$ and $A_{1}$. The initial conditions $A_{j}$, $j=0,1, \ldots, n-1$, and coefficient $B$ are assumed to be independent real $2-\mathrm{RVs}$.

The Airy differential equations and its related functions [115] are applied in several fields such as fluid mechanics, elasticity, quantum physics and so on. It has been studied separately in the context of deterministic FDEs $[3,117,6]$ and in the RDEs [42], however to the best of our knowledge, its mathematical study combining both fractional and random calculus, i.e., treating it as a RFDEs has not been undertaken yet, which is the motivation of this scientific chapter.

The main goal of this chapter is to establish conditions on $\operatorname{RVs} A_{j}, j=0,1, \ldots, n-1$, and $B$ in order to construct a m.s. solution to problem (6.1) in the following form

$$
Y(t)=\sum_{j=0}^{n-1} Y_{j}(t) \quad \text { with } \quad Y_{j}(t)=\sum_{m=0}^{\infty} X_{m, j} t^{\gamma m+j}
$$

In the above expression, $X_{m, j}$ is a sequence of 2-RVs to be determined, and $\gamma:=\alpha+\beta$. Observe that $\gamma>n-1$ because $n-1<\alpha \leq n$ and $\beta>0$. As the solution of random IVP (6.1) is a SP, an important target is to compute its statistical properties as well. Specifically, as our study is based on m.s. random calculus corresponding to $\mathrm{L}^{2}(\Omega)$, we will construct approximations for the two first statistical moments (the mean and the variance) of the solution SP $Y(t)$. At this point it is worth pointing out that in this way we will give our results in the biggest 
$\mathrm{L}^{p}(\Omega)$-type Lebesgue space, since it is known that $\mathrm{L}^{q}(\Omega) \subset \mathrm{L}^{p}(\Omega), 1 \leq p \leq q \leq \infty$, and taking $p=2$ is guaranteed the existence of variance (or equivalently of randomness). As an interesting application of obtaining approximations of the mean and of the variance, we take advantage of Principle of Maximum Entropy to construct the probability density function of the solution.

This chapter is organized as follows: In Section 6.2, first we construct a random generalized power series solution of the form (6.2) to the random fractional IVP (6.1) and then we provide sufficient conditions on the coefficient $B$ and the initial conditions $A_{j}, 0 \leq j \leq n-1$, in order to guarantee the m.s. convergence of that random series. Section 6.3 is addressed to compute the main statistical properties of the solution SP, namely, the mean, the variance and the covariance. Section 6.5 is devoted to show a set of numerical examples where our theoretical results are illustrated. Section 6.4 shows, by means of a detailed example, how to construct reliable approximations of the probability density function of the solution SP to the random fractional Airy differential equation by combining the knowledge of the mean and of the variance together with the Principle of Maximum Entropy. In this manner we are able to provide a full probabilistic description of the solution SP at each time instant. Our conclusions are shown in Section 6.6.

\subsection{Constructing the solution stochastic process}

As it will be seen right now, we will obtain the solution to the random fractional IVP (6.1) following a constructive reasoning. This process will require to legitimate certain operational rules, in the m.s. sense. For the sake of clarity, we start this section by rigorously proving these rules. To this purpose, we will apply [109, Property (4.126), page 96] to calculate the m.s. derivative of the product of a differentiable deterministic function and a m.s. differentiable SP, and Proposition 2.4 to differentiate a series of m.s. SPs.

Our first step will be to rigorously legitimate that the first m.s. derivative of the SP $Y_{j}(t)$, defined in (6.2) for $t>0$, is given by

$$
\frac{\mathrm{d}}{\mathrm{d} t}\left[Y_{j}(t)\right]=\sum_{m=0}^{\infty} X_{m, j} \frac{\mathrm{d}}{\mathrm{d} t}\left[t^{\gamma m+j}\right], \quad j=0,1, \ldots, n-1 .
$$


Indeed, take $t_{0}>0$ fixed and for each $j$, let $U_{m}(t)=X_{m, j} t^{\gamma m+j}$ being $X_{m, j} \in$ $\mathrm{L}^{2}(\Omega)$. Then applying [109, property (4.126), page 96] to $f(t)=t^{\gamma m+j}$ and $X(t)=X_{m, j}$, one gets that $U_{m}(t)$ is m.s. differentiable at $t_{0}$ being $\left.\frac{\mathrm{d}}{\mathrm{d} t}\left[U_{m}(t)\right]\right|_{t=t_{0}}=$ $\left.X_{m, j} \frac{\mathrm{d}}{\mathrm{d} t}\left[t^{m \gamma+j}\right]\right|_{t=t_{0}}$ its m.s. derivative for every $m \geq 0$. Moreover, if $X_{m, j} \in \mathrm{L}^{2}(\Omega)$ then clearly the sequence of SPs $\left\{U_{m}(t): m \geq 0\right\}$ is m.s. continuous at $t_{0}$. Take $\mathcal{T}$ an interval in $\mathbb{R}^{+}$and define $U(t)=\sum_{m \geq 0} U_{m}(t)$. If this latter sum is m.s. convergent and $\sum_{n \geq 0} U_{m}^{\prime}(t)$ converges uniformly in the m.s. sense for each $t \in \mathcal{T}$, then Proposition 2.4 it is guaranteed that (6.3) holds. At this point is important to underline that we have implicitly assumed that $X_{m, j} \in \mathrm{L}^{2}(\Omega)$. Later we will compute explicitly coefficients $X_{m, j}$ in terms of $A_{j}, j=0,1, \ldots, n-1$ and $B$, and then assuming appropriate hypotheses on input data we will check that $X_{m, j} \in \mathrm{L}^{2}(\Omega)$. Following an analogous reasoning, it can easily be shown that

$$
\frac{\mathrm{d}^{n}}{\mathrm{~d} t^{n}}\left[Y_{j}(t)\right]=\sum_{m=0}^{\infty} X_{m, j} \frac{\mathrm{d}^{n}}{\mathrm{~d} t^{n}}\left[t^{\gamma m+j}\right], \quad n \geq 1,
$$

being $n$ a fixed positive integer.

Using the fractional derivative of the power function $t^{\nu}$ given by [52, Example 3.1]

$$
{ }^{C} D_{0^{+}}^{\alpha} t^{\nu}=\left\{\begin{array}{cl}
t^{\nu-\alpha} \frac{\Gamma(\nu+1)}{\Gamma(\nu+1-\alpha)} & \text { if } \quad \nu>n-1, \\
0 & \text { if } \quad \nu=0,1, \ldots, n-1,
\end{array} \quad n=-\lfloor-\alpha\rfloor, \alpha>0,\right.
$$


and (6.4), one derives

$$
\begin{aligned}
& \left({ }^{C} D_{0^{+}}^{\alpha} Y\right)(t)=\left({ }^{C} D_{0^{+}}^{\alpha} \sum_{j=0}^{n-1} Y_{j}\right)(t) \\
& =\sum_{j=0}^{n-1}\left({ }^{C} D_{0^{+}}^{\alpha} Y_{j}\right)(t) \\
& =\sum_{j=0}^{n-1}\left(\frac{1}{\Gamma(n-\alpha)} \int_{0}^{t}(t-u)^{n-\alpha-1} \frac{\mathrm{d}^{n}}{\mathrm{~d} u^{n}}\left[Y_{j}(u)\right] \mathrm{d} u\right) \\
& \text { by (6.4) } \\
& =\sum_{j=0}^{n-1}\left(\frac{1}{\Gamma(n-\alpha)} \int_{0}^{t}(t-u)^{n-\alpha-1} \sum_{m=0}^{\infty} X_{m, j} \frac{\mathrm{d}^{n}}{\mathrm{~d} u^{n}}\left[u^{\gamma m+j}\right] \mathrm{d} u\right) \\
& =\sum_{j=0}^{n-1}\left(\sum_{m=0}^{\infty} X_{m, j} \frac{1}{\Gamma(n-\alpha)} \int_{0}^{t}(t-u)^{n-\alpha-1} \frac{\mathrm{d}^{n}}{\mathrm{~d} u^{n}}\left[u^{\gamma m+j}\right] \mathrm{d} u\right) \\
& =\sum_{j=0}^{n-1}\left(\sum_{m=0}^{\infty} X_{m, j}{ }^{C} D_{0^{+}}^{\alpha} t^{\gamma m+j}\right) \\
& \text { by (6.5) } \\
& =\sum_{j=0}^{n-1} \sum_{m=1}^{\infty} X_{m, j} \frac{\Gamma(\gamma m+j+1)}{\Gamma(\gamma m+j+1-\alpha)} t^{\gamma m+j-\alpha} \\
& =\sum_{m=0}^{\infty} \sum_{j=0}^{n-1} X_{m+1, j} \frac{\Gamma(\gamma(m+1)+j+1)}{\Gamma(\gamma(m+1)+j+1-\alpha)} t^{\gamma(m+1)+j-\alpha} \text {. }
\end{aligned}
$$

Notice that when applying (6.4), we have used that $\gamma=\alpha+\beta>n-1$.

Hence, taking into account that $\gamma=\alpha+\beta$, one obtains

$$
\begin{gathered}
\left({ }^{C} D_{0^{+}}^{\alpha} Y\right)(t)-B t^{\beta} Y(t)=\sum_{m=0}^{\infty} \sum_{j=0}^{n-1} X_{m+1, j} \frac{\Gamma(\gamma(m+1)+j+1)}{\Gamma(\gamma(m+1)+j+1-\alpha)} t^{\gamma(m+1)+j-\alpha} \\
-B t^{\beta} \sum_{m=0}^{\infty} \sum_{j=0}^{n-1} X_{m, j} t^{\gamma m+j} \\
=\sum_{m=0}^{\infty} \sum_{j=0}^{n-1}\left[X_{m+1, j} \frac{\Gamma(\gamma(m+1)+j+1)}{\Gamma(\gamma(m+1)+j+1-\alpha)}-B X_{m, j}\right] t^{\gamma m+j+\beta} .
\end{gathered}
$$


So, if we choose

$$
X_{m+1, j}=B X_{m, j} \frac{\Gamma(\gamma(m+1)+j+1-\alpha)}{\Gamma(\gamma(m+1)+j+1)}, \quad m=0,1, \ldots
$$

in (6.7), it is guarantee that $Y(t)$ defined by (6.2) will satisfy the RFDE $\left({ }^{C} D_{0^{+}}^{\alpha} Y\right)(t)-$ $B t^{\beta} Y(t)=0$. Taking into account that $Y^{(j)}(0)=X_{0, j}=A_{j}$ for $j=0,1, \ldots, n-1$, the recursion for the sequence of coefficients $X_{m, j}$ gives

$$
X_{m, j}=B^{m} A_{j} G_{m, j}, \quad G_{m, j}:=\prod_{k=1}^{m} \frac{\Gamma(k \gamma+j+1-\alpha)}{\Gamma(k \gamma+j+1)}>0, \quad m \geq 0,
$$

where as usual we implicitly assume that $\prod_{k=1}^{m} y_{k}=1$ when $m=0$. Therefore

$$
Y(t)=\sum_{j=0}^{n-1} Y_{j}(t)=\sum_{j=0}^{n-1}\left(\sum_{m=0}^{\infty} B^{m} A_{j} G_{m, j} t^{\gamma m+j}\right)
$$

is a m.s. solution of (6.1), provided that, for each $j$, the $\operatorname{SPs} Y_{j}(t)$ and $\frac{\mathrm{d}^{i} Y_{j}(t)}{\mathrm{d} t^{i}}, i=$ $1,2, \ldots, n$ are m.s. convergent and m.s. uniformly convergent on $\mathcal{T}$, respectively. In order to justify that these conditions fulfil, we will assume the following hypotheses for the random inputs $A_{j}, j=0,1,2, \ldots, n-1$ and $B$ :

K1: There exist constants $\eta, \mathcal{H}>0$ and $p \geq 0$ and $m, m_{0}$ integers such that

$$
\left\|B^{m}\right\|_{2} \leq \eta \mathcal{H}^{m-1}((m-1) !)^{p}, \quad \forall m: m \geq m_{0} \geq 1 .
$$

K2: For every $j, j=0,1, \ldots, n-1, A_{j}$ and $B$ are independent RVs.

As a consequence of hypotheses $\mathbf{K} \mathbf{1}$ and $\mathbf{K} \mathbf{2}$, one gets

$$
\left\|B^{m} A_{j} G_{m, j} t^{\gamma m+j}\right\|_{2} \leq \eta \mathcal{H}^{m-1}((m-1) !)^{p}\left\|A_{j}\right\|_{2} G_{m, j} t^{\gamma m+j}:=\delta_{m, j}(t),
$$

for $j=0,1, \ldots, n-1, m \geq 1$ integer and $t>0$. For every $j$ fixed, we study the domain of convergence of the infinite sum $\sum_{m=0}^{\infty} \delta_{m, j}(t)$ by applying the ratio test and the Stirling formula (2.12). 
The approximation $\Gamma(x+1) \approx x^{x} \mathrm{e}^{-x} \sqrt{2 \pi x}$ as $x \rightarrow \infty$ will be used too. Recall that $\gamma=\alpha+\beta$.

$$
\begin{aligned}
& \lim _{m \rightarrow \infty} \frac{\delta_{m+1, j}(t)}{\delta_{m, j}(t)} \\
& =\lim _{m \rightarrow \infty} \mathcal{H} m^{p} \frac{\Gamma((m+1) \gamma+j+1-\alpha)}{\Gamma((m+1) \gamma+j+1)} t^{\gamma} \\
& =\lim _{m \rightarrow \infty} \mathcal{H} m^{p}\left[\frac{(m+1) \gamma+j-\alpha}{(m+1) \gamma+j}\right]^{(m+1) \gamma+j-\alpha}\left(\frac{1}{(m+1) \gamma+j}\right)^{\alpha} \\
& \left.\cdot \mathrm{e}^{\alpha} \sqrt{\frac{(m+1) \gamma+j-\alpha}{(m+1) \gamma+j} t^{\alpha+\beta}}\right]_{m \rightarrow \infty}\left[\frac{(m+1) \gamma+j-\alpha}{(m+1) \gamma+j}\right]^{\beta+j}\left(\frac{1}{(m+1) \gamma+j}\right)^{\alpha} \\
& =\lim _{m \rightarrow} \mathcal{H}\left[\frac{(m+1) \gamma+j-\alpha}{(m+1) \gamma+j}\right. \\
& \cdot \mathrm{e}^{\alpha} \sqrt{\frac{(m+1) \gamma+j-\alpha}{(m+1) \gamma+j}} t^{\alpha+\beta} .
\end{aligned}
$$

Now taking into account that

$$
\lim _{m \rightarrow+\infty}\left[\frac{(m+1) \gamma+j-\alpha}{(m+1) \gamma+j}\right]^{m \gamma}=\mathrm{e}^{-\alpha}
$$

and

$$
\lim _{m \rightarrow+\infty} \frac{(m+1) \gamma+j-\alpha}{(m+1) \gamma+j}=1, \quad t \geq 0
$$

one gets

$$
\begin{aligned}
\lim _{m \rightarrow+\infty} \frac{\delta_{m+1}(t)}{\delta_{m}(t)} & =t^{\alpha+\beta} \mathcal{H} \lim _{m \rightarrow+\infty} \frac{m^{p}}{((m+1)(\alpha+\beta)+j)^{\alpha}} \\
& =\left\{\begin{array}{c}
0 \quad \text { if } 0 \leq p<\alpha, \\
\mathcal{H} t^{\beta}\left(\frac{t}{\alpha+\beta}\right)^{\alpha} \text { if } p=\alpha .
\end{array}\right.
\end{aligned}
$$


So, we have proved that the majorizing series $\sum_{m=0}^{\infty} \delta_{m, j}(t)$ converges for every $t$ in $\mathcal{D}$, where

$$
\mathcal{D}=\left\{\begin{array}{cll}
{[0, \infty)} & \text { if } & 0 \leq p<\alpha, \\
{\left[0, \frac{(\alpha+\beta)^{\frac{\alpha}{\alpha+\beta}}}{\mathcal{H}^{\frac{1}{\alpha+\beta}}}\left[\begin{array}{ll}
\text { if } & p=\alpha .
\end{array}\right.\right.} &
\end{array}\right.
$$

Summarizing, we have found sufficient conditions for the 2SP $Y(t)$ to be a m.s. solution of the IVP given in (6.1). The next result states our findings.

Theorem 6.1 If the RVs $A_{j}, j=0,1, \ldots, n-1$, and $B$ satisfy conditions $\boldsymbol{K} \mathbf{1}$ and K2, then the $S P$

$$
Y(t)=\sum_{j=0}^{n-1}\left(\sum_{m=0}^{\infty} B^{m} A_{j} G_{m, j} t^{\gamma m+j}\right), \quad \gamma=\alpha+\beta,
$$

where

$$
G_{m, j}=\prod_{k=1}^{m} \frac{\Gamma(k \gamma+j+1-\alpha)}{\Gamma(k \gamma+j+1)}, \quad m=0,1,2, \ldots,
$$

is a m.s. solution of problem (6.1) for $t \in \mathcal{T}=\left[t_{1}, t_{2}\right] \subset \mathcal{D} \subset \mathbb{R}$, being $\mathcal{D}$ given in (6.11).

Notice that the domain of convergence of $Y(t)$ depends on the relation between the order of the fractional derivative, $\alpha>0$, and the value of $p$, involved in the hypothesis K1, which only affects the random coefficient $B$.

\subsection{Probabilistic properties of the solution stochastic process}

Once we have constructed a solution SP, $Y(t)$, to the random fractional IVP (6.1), an important issue is to compute its main statistical properties, such as the mean $(\mathbb{E}[Y(t)])$, variance $(\mathbb{V}[Y(t)])$ and covariance $(\mathbb{C o v}[Y(t), Y(s)])$ functions. Since the solution SP, $Y(t)$, has been constructed via the infinite series (6.12)-(6.13), it must be truncated to keep feasible the computational burden. Thus, for a positive integer $M$, hereinafter we will consider the truncated series, $Y_{M}(t)$, of $Y(t)$, defined by

$$
Y_{M}(t)=\sum_{j=0}^{n-1}\left(\sum_{m=0}^{M} B^{m} A_{j} G_{m, j} t^{\gamma m+j}\right) .
$$


To this end, the Proposition 2.10 will play a key role and it also justifies why we have made the decision to conduct our study of uncertainty in the random fractional IVP (6.1) using the m.s. convergence instead of considering other stochastic convergences like almost surely, in probability or in distribution. Notice that we can take advantage of this result because m.s. convergence of the infinite series defining $Y(t)$ for $t>0$ has been rigorously established in Theorem 6.1.

Taking the expectation operator in (6.14) and using its linearity and hypothesis $\mathbf{K 2}$, it is clear that the mean of $Y_{M}(t)$ is given by

$$
\mathbb{E}\left[Y_{M}(t)\right]=\sum_{j=0}^{n-1}\left(\sum_{m=0}^{M} \mathbb{E}\left[B^{m}\right] \mathbb{E}\left[A_{j}\right] G_{m, j} t^{\gamma m+j}\right) .
$$

Notice that in order to compute approximations of the expectation to the solution SP $Y(t)$ of the random IVP $(6.1)$ via $Y_{M}(t)$, the expectation of the initial conditions, $A_{j}, 0 \leq j \leq n-1$, and the moments with respect to (w.r.t.) the origin up to order $M$ of the random coefficient $B$ are just required.

Now, we are interested in computing an approximation of the variance of $Y_{M}(t)$. To this end, and for the sake of generality, we first calculate its cross-covariance 


$$
\begin{aligned}
& \mathbb{C}_{Y_{M}, Y_{N}}(t, s):=\mathbb{C o v}\left[Y_{M}(t), Y_{N}(s)\right] \\
& =\sum_{j=0}^{n-1} \sum_{k=0}^{n-1} \operatorname{Cov}\left[\sum_{r=0}^{M} B^{r} A_{j} G_{r, j} t^{\gamma r+j}, \sum_{m=0}^{N} B^{m} A_{k} G_{m, k} s^{\gamma m+k}\right] \\
& =\sum_{j=0}^{n-1} \sum_{k=0}^{n-1} \sum_{r=0}^{M} \sum_{m=0}^{N} \operatorname{Cov}\left[B^{r} A_{j}, B^{m} A_{k}\right] G_{r, j} G_{m, k} t^{\gamma r+j} s^{\gamma m+k} \\
& =\sum_{j=0}^{n-1} \sum_{k=0}^{n-1} \sum_{r=0}^{N} \sum_{m=0}^{M}\left(\mathbb{E}\left[A_{j} A_{k} B^{m+r}\right]-\mathbb{E}\left[B^{r} A_{j}\right] \mathbb{E}\left[B^{m} A_{k}\right]\right) \\
& \text { - } G_{r, j} G_{m, k} t^{\gamma r+j} s^{\gamma m+k} \\
& =\sum_{j=0}^{n-1} \sum_{k=0}^{n-1} \sum_{r=0}^{N} \sum_{m=0}^{M}\left(\mathbb{E}\left[A_{j}\right] \mathbb{E}\left[A_{k}\right] \mathbb{E}\left[B^{m+r}\right]-\mathbb{E}\left[A_{j}\right] \mathbb{E}\left[A_{k}\right] \mathbb{E}\left[B^{m}\right] \mathbb{E}\left[B^{r}\right]\right) \\
& \text { - } G_{r, j} G_{m, k} t^{\gamma r+j} s^{\gamma m+k} \\
& =\sum_{j=0}^{n-1} \sum_{k=0}^{n-1} \sum_{r=0}^{N} \sum_{m=0}^{M} \mathbb{E}\left[A_{j}\right] \mathbb{E}\left[A_{k}\right]\left(\mathbb{E}\left[B^{m+r}\right]-\mathbb{E}\left[B^{m}\right] \mathbb{E}\left[B^{r}\right]\right) \\
& \text { - } G_{r, j} G_{m, k} t^{\gamma r+j} s^{\gamma m+k} \\
& =\sum_{j=0}^{n-1} \sum_{k=0}^{n-1} \sum_{r=0}^{N} \sum_{m=0}^{M} \mathbb{E}\left[A_{j}\right] \mathbb{E}\left[A_{k}\right] \operatorname{Cov}\left[B^{m}, B^{r}\right] G_{r, j} G_{m, k} t^{\gamma r+j} s^{\gamma m+k},
\end{aligned}
$$

where we have used the bilinearity of the covariance, its definition and the (mutually) independence of RVs $A_{0}, A_{1}, \ldots, B$, assumed in hypothesis $\mathbf{K 2}$. In the particular case that $M=N$ and $t=s$ in (6.16), one obtains the variance of the $Y_{M}(t)$ 


$$
\begin{aligned}
\mathbb{V}\left[Y_{M}(t)\right]= & \operatorname{Cov}\left[Y_{M}(t), Y_{M}(t)\right] \\
= & \sum_{j=0}^{n-1} \sum_{k=0}^{n-1} \sum_{r=0}^{M} \sum_{m=0}^{M} \mathbb{E}\left[A_{j}\right] \mathbb{E}\left[A_{k}\right] \mathbb{C o v}\left[B^{m}, B^{r}\right] G_{r, j} G_{m, k} t^{\gamma r+j} s^{\gamma m+k} \\
= & \sum_{j=0}^{n-1} \sum_{k=0}^{n-1} \sum_{r=0}^{M} \sum_{m=0}^{M} \mathbb{E}\left[A_{j}\right] \mathbb{E}\left[A_{k}\right]\left(\mathbb{E}\left[B^{m+r}\right]-\mathbb{E}\left[B^{m}\right] \mathbb{E}\left[B^{r}\right]\right) \\
& \cdot G_{r, j} G_{m, k} t^{\gamma r+j} s^{\gamma m+k} .
\end{aligned}
$$

Notice that in order to compute approximations of the variance to the solution SP $Y(t)$ of the random IVP (6.1) via $Y_{M}(t)$, the expectation of the initial conditions, $A_{j}, 0 \leq j \leq n-1$, as well as the moments w.r.t. the origin up to order $2 M$ of the random coefficient $B$ are just required.

Remark 6.1 Now, we make a constructive comment related to hypothesis $\mathbf{K} 2$. In Section 6.2, to prove m.s. convergence of the solution $S P Y(t)$ given by (6.12), hypothesis $\mathbf{K} 2$ could be relaxed assuming that $A_{j}$ and $B$ are independent $R V$ s for every $j: 0 \leq j \leq n-1$ instead (to see this, just check inequality (6.8) and apply [63, Theorem 3, page 92]). However, to keep a common assumption throughout the whole presentation of the dissertation we have embraced (mutually) independence among all involved RVs in $\mathbf{K 2}$, which is used to give a handy expression for the covariance, and thus for the variance too (see expressions (6.16) and (6.17), respectively).

Remark 6.2 In the proof of Theorem 6.1, the set $\mathcal{D}$ is the interval where the deterministic majorizing series, $\sum_{m=0}^{\infty} \delta_{m, j}(t)$, for the solution $Y(t)$ is convergent. As a consequence, the solution $Y(t)$ is m.s. convergent on $\mathcal{D}$, since $Y(t)$ was majorized by $\sum_{m=0}^{\infty} \delta_{m, j}(t)$ with the norm $\|\cdot\|_{2}$. However, the interval of convergence of $Y(t)$ may be larger than $\mathcal{D}$. As a result of Proposition 2.10, the above approximations of the mean, $\mathbb{E}\left[Y_{M}(t)\right]$, and the variance, $\mathbb{V}\left[Y_{M}(t)\right]$, of $Y(t)$ are also convergent on $\mathcal{D}$. As in the case that $p=\alpha$, the interval $\mathcal{D}$ is bounded, $\mathcal{D}=\left[0, \frac{(\alpha+\beta)^{\frac{\alpha}{\alpha+\beta}}}{\mathcal{H}^{\frac{1}{\alpha+\beta}}}[\right.$, the approximations of the variance (and thus of the mean) may converge in larger intervals. This issue will be illustrated later in Example 6.3. 


\subsection{Approximating the probability density function via the Principle of Maximum Entropy}

So far, we have computed approximations for the mean and for the variance of the solution SP, $Y(t)$, given by (6.12). However, more probabilistic information is occasionally required to get a complete statistical description of $Y(t)$. As it has been indicated in Section 4.1, the probability density function (PDF) allows us to obtain a full probabilistic description of the solution SP $Y(t)$. In this section, we use information of the estimations for the mean and the variance $((6.15)$ and (6.17), respectively) to construct approximations of the PDF of $Y(t)$, denoted as $f_{Y(t)}(y, t)$.

To do it, we will approximate $f_{Y(t)}(y, t)$ by calculating the PDF of the approximation $Y_{M}(t)$, given by (6.14). Let us denote by $f_{Y_{M}(t)}(y, t)$ the 1-PDF of $Y_{M}(t)$. To achieve this goal, we will combine the Principle of Maximum Entropy (PME), [91], with the approximations for the mean and for the variance obtained in (6.15) and (6.17), respectively.

The mathematical concept of entropy is a measurement of uncertainty. It defines the lack of knowledge of a RV, which has been built on the basis of limited probabilistic information. The larger the uncertainty of the RV, the larger its entropy [91, Section 2.2]. Here, we will apply the so-called differential entropy or Shannon's entropy, $S_{R}$. Given a RV, say $R, S_{R}$ is defined by

$$
S_{R}=-\int_{\mathcal{D}(R)} f_{R}(r) \log \left(f_{R}(r)\right) \mathrm{d} r,
$$

where $\mathcal{D}(R)$ denotes the domain of $R$ and $f_{R}$ its PDF. In our setting, entropy will be used to construct approximations of the PDF, $f_{Y_{M}(t)}(y, t)$, using the available information of $Y_{M}(t)$, in this case, the approximations for the mean, (6.15), and for the variance, $(6.17)$.

We first fix a time instant $t=\hat{t}$ and an order of truncation $M$ large enough to guarantee that

$$
\begin{aligned}
\mathbb{E}\left[Y_{M}(\hat{t})\right] & \approx \mathbb{E}[Y(\hat{t})]=m_{1}(\hat{t}) \equiv m_{1}, \\
\mathbb{E}\left[\left(Y_{M}(\hat{t})\right)^{2}\right] & \approx \mathbb{E}\left[(Y(\hat{t}))^{2}\right]=m_{2}(\hat{t}) \equiv m_{2},
\end{aligned}
$$


i.e., that the approximations of the two first moments with respect to the origin (also the variance) are close enough. Observe that this fact has been legitimated in Section 6.3 because of Proposition 2.10. Also notice that for convenience, in (6.19) we have hidden the time dependence in the notation for $m_{i}, i=1,2$. Then, we adapt the PME, that is typically applied within the context of RVs $[100,75$, $62]$, to our setting.

Let $Y_{M}(\hat{t})$ be the continuous RV that approximates $Y(\hat{t})$. To facilitate the notation $Y_{M}(t)$ and $f_{Y_{M}(t)}(y, t)$ will be denoted as $Y_{M}$ and $f_{Y_{M}}(y)$, respectively. The PME consists of determining the function $f_{Y_{M}}(y)$ that corresponds to the maximal randomness (maximal entropy) with the minimal information available. To do that, assuming that $f_{R}(r)$ exhibited in (6.18) represents the PDF of $Y_{M}$, i.e. $f_{R}(r)=f_{Y_{M}}$, PME seeks the function $f_{Y_{M}}$ that maximizes $S_{Y_{M}}$ (defined in (6.18)), capturing the lowest knoledge of the RV $Y_{M}$. In our case, as $f_{Y_{M}}$ represents a PDF, their integral over the domain of $Y_{M}$ must be the unit and the two first theoretical moments of $Y(t)$, calculated via the $\mathrm{PDF}$, also match the corresponding values of $m_{1}$ and $m_{2}$ (obtained via the numerical approximations), i.e.

$$
\begin{gathered}
\int_{\mathcal{D}\left(Y_{M}\right)} f_{Y_{M}}(y) \mathrm{d} y=1, \\
\int_{\mathcal{D}\left(Y_{M}\right)} y f_{Y_{M}}(y) \mathrm{d} y=m_{1}, \\
\int_{\mathcal{D}\left(Y_{M}\right)} y^{2} f_{Y_{M}}(y) \mathrm{d} y=m_{2},
\end{gathered}
$$

where $\mathcal{D}\left(Y_{M}\right)$ denotes the domain of the RV $Y_{M}$, which is unknown in practice. To overcome this lack of information in the domain of integration, here we use the Bienaymé-Chebysshev's inequality [36, page 122] to approximate $\mathcal{D}\left(Y_{M}\right)$. According to this important result, we can assure that the interval $\left[a_{1}, a_{2}\right]$, with $a_{1}=\mathbb{E}\left[Y_{M}\right]-10 \sqrt{\mathbb{V}\left[Y_{M}\right]}$ and $a_{2}=\mathbb{E}\left[Y_{M}\right]+10 \sqrt{\mathbb{V}\left[Y_{M}\right]}$, will contain all the outcomes of $Y_{M}$ with a probability of $99.9 \%$, regardless the distribution of $Y_{M}$. So, in our subsequent numerical computations we will take $\mathcal{D}\left(Y_{M}\right) \approx\left[a_{1}, a_{2}\right]$. The $\mathrm{PDF} f_{Y_{M}}(y)$ is calculated by maximizing Shannon entropy of the RV $Y_{M}$. To this end, we apply the variational extension of classical Lagrange multiplier method 
[91]. Hence, for a fixed $t=\hat{t}$ we search a function, $f_{Y_{M}}:\left[a_{1}, a_{2}\right] \longrightarrow \mathbb{R}$, such that

$$
\begin{aligned}
\mathcal{L}\left(f_{Y_{M}}, \lambda_{0}, \lambda_{1}, \lambda_{2}\right)= & -\int_{a_{1}}^{a_{2}} f_{Y_{M}}(y) \log \left(f_{Y_{M}}(y)\right) \mathrm{d} y+\lambda_{0}\left(1-\int_{a_{1}}^{a_{2}} f_{Y_{M}}(y) \mathrm{d} y\right) \\
& +\lambda_{1}\left(\mathbb{E}\left[Y_{M}\right]-\int_{a_{1}}^{a_{2}} y f_{Y_{M}}(y) \mathrm{d} y\right) \\
& +\lambda_{2}\left(\mathbb{E}\left[\left(Y_{M}\right)^{2}\right]-\int_{a_{1}}^{a_{2}} y^{2} f_{Y_{M}}(y) \mathrm{d} y\right) \\
= & -\int_{a_{1}}^{a_{2}} f_{Y_{M}}(y)\left(\log \left(f_{Y_{M}}(y)\right)+\sum_{i=0}^{2} \lambda_{i} y^{i}\right) \mathrm{d} y \\
& ++\lambda_{0}+\lambda_{1} \mathbb{E}\left[Y_{M}\right]+\lambda_{2} \mathbb{E}\left[\left(Y_{M}\right)^{2}\right],
\end{aligned}
$$

where $\lambda_{i}, i=0,1,2$, are the so-called Lagrange multipliers. Using variational calculus, we impose the four conditions:

$$
\begin{aligned}
& \frac{\partial \mathcal{L}\left(f_{Y_{M}}, \lambda_{0}, \lambda_{1}, \lambda_{2}\right)}{\partial f_{Y_{M}}}=0, \\
& \frac{\partial \mathcal{L}\left(f_{Y_{M}}, \lambda_{0}, \lambda_{1}, \lambda_{2}\right)}{\partial \lambda_{0}}=0, \\
& \frac{\partial \mathcal{L}\left(f_{Y_{M}}, \lambda_{0}, \lambda_{1}, \lambda_{2}\right)}{\partial \lambda_{1}}=0, \\
& \frac{\partial \mathcal{L}\left(f_{Y_{M}}, \lambda_{0}, \lambda_{1}, \lambda_{2}\right)}{\partial \lambda_{2}}=0 .
\end{aligned}
$$

The first condition yields

$$
\left.\frac{\partial \mathcal{L}\left(f_{Y_{M}}, \lambda_{0}, \lambda_{1}, \lambda_{2}\right)}{\partial f_{Y_{M}}}=-\int_{a_{1}}^{a_{2}}\left(1+\log \left(f_{Y_{M}}(y)\right)\right)+\sum_{i=0}^{2} \lambda_{i} y^{i}\right) \mathrm{d} y=0 .
$$

Obviously, this condition holds when $\left.1+\log \left(f_{Y_{M}}(y)\right)\right)+\sum_{i=0}^{2} \lambda_{i} y^{i}=0$. This yields

$$
f_{Y_{M}}(y)=\mathbb{1}_{\left[a_{1}, a_{2}\right]} \mathrm{e}^{-1-\lambda_{0}-\lambda_{1} y-\lambda_{2} y^{2}},
$$


where $\mathbb{1}_{\left[a_{1}, a_{2}\right]}$ denotes the characteristic function on the interval $\left[a_{1}, a_{2}\right] \approx \mathcal{D}\left(Y_{M}\right)$, given by

$$
\mathbb{1}_{\left[a_{1}, a_{2}\right]}(y)=\left\{\begin{array}{lc}
1, & a_{1} \leq y \leq a_{2} \\
0, & \text { otherwise }
\end{array}\right.
$$

On the other hand, with the remaining conditions of (6.21), we can compute the values of Lagrange multipliers $\lambda_{0}, \lambda_{1}, \lambda_{2}$, solving the nonlinear system

$$
\left\{\begin{array}{l}
\frac{\partial \mathcal{L}\left(f_{Y_{M}}, \lambda_{0}, \lambda_{1}, \lambda_{2}\right)}{\partial \lambda_{0}}=0 \longrightarrow \int_{a_{1}}^{a_{2}} \mathrm{e}^{-1-\lambda_{0}-\lambda_{1} y-\lambda_{2} y^{2}} \mathrm{~d} y=1, \\
\frac{\partial \mathcal{L}\left(f_{Y_{M}}, \lambda_{0}, \lambda_{1}, \lambda_{2}\right)}{\partial \lambda_{1}}=0 \longrightarrow \int_{a_{1}}^{a_{2}} y \mathrm{e}^{-1-\lambda_{0}-\lambda_{1} y-\lambda_{2} y^{2}} \mathrm{~d} y=m_{1}, \\
\frac{\partial \mathcal{L}\left(f_{Y_{M}}, \lambda_{0}, \lambda_{1}, \lambda_{2}\right)}{\partial \lambda_{2}}=0 \longrightarrow \int_{a_{1}}^{a_{2}} y^{2} \mathrm{e}^{-1-\lambda_{0}-\lambda_{1} y-\lambda_{2} y^{2}} \mathrm{~d} y=m_{2} .
\end{array}\right.
$$

This is a non-linear system of equations involving the Gauss error function erf[·] when the integrals are calculated. Therefore, in practice numerical methods, such as Newton-Raphson, are required to determine its solution $\left(\lambda_{0}, \lambda_{1}, \lambda_{2}\right)$. Once these values have been computed, $f_{Y_{M}}$ is easily obtained by expression (6.22).

\subsection{Numerical examples}

This section is addressed to present some numerical examples where our previous theoretical findings are illustrated. We will compute approximations to the mean $\left(\mathbb{E}\left[Y_{M}(t)\right]\right)$ and the variance/standard deviation $\left(\mathbb{V}\left[Y_{M}(t)\right] / \sigma\left[Y_{M}(t)\right]\right)$ of the solution SP at different time instants $t$ by increasing the order of truncation $M$ using the expressions (6.15) and (6.17), respectively. Considering $\mathrm{M}$ enough to guarantee that $\mathbb{E}\left[Y_{M}(t)\right] \approx \mathbb{E}[Y(t)]$ and $\mathbb{V}\left[Y_{M}(t)\right] \approx \mathbb{V}[Y(t)]$, in the first example, approximations for the 1-PDF of $Y_{M}(t)$ have been constructed by PME approach described in Section 6.4. The results are presented via graphical representations or numerical tables of these statistical moments. In some of the examples, we have also calculated the relative error of consecutive approximations of these two 
statistical moments with respect to the order of truncation

$$
\begin{gathered}
\operatorname{RE}(\text { Mean })(t ; M)=\left|\frac{\mathbb{E}\left[Y_{M+1}(t)\right]-\mathbb{E}\left[Y_{M}(t)\right]}{\mathbb{E}\left[Y_{M}(t)\right]}\right|, \\
\operatorname{RE}(\text { Variance })(t ; M)=\left|\frac{\mathbb{V}\left[Y_{M+1}(t)\right]-\mathbb{V}\left[Y_{M}(t)\right]}{\mathbb{V}\left[Y_{M}(t)\right]}\right| .
\end{gathered}
$$

The examples are devised to illustrate the two possible situations with respect the domain of convergence established in Theorem 6.1. Observe that both cases depend on the relationship between $\alpha$ (order of the fractional derivative) and $p$ (parameter involved in hypothesis $\mathbf{K} \mathbf{1}$ and related to the behaviour of moments w.r.t. the origin of RV $B$ ). The case $p<\alpha$ is illustrated in the Example 6.2, while the case $p=\alpha$ is analysed in the Example 6.3. As the IVP (6.1) corresponds to the random classical Airy differential equation when $\alpha=2$ and $\beta=1$, we will begin with Example 6.1 where this equation is analysed taking values of parameter $\alpha$ such that $\alpha \rightarrow 2^{-}$. Finally, we point out that in these three examples we have considered a wide variety of probability distributions for the random coefficient $B$ and different numerical values for the moments of initial conditions $A_{j}$, with the aim of showing the generality when applying the theoretical results previously established. As required in hypothesis K2, in the following examples we will assume that $B$ and the involved initial conditions $A_{j}$ are independent RVs.

Example 6.1 In order to check that the our theoretical findings for the random Airy fractional differential equation are consistent with the corresponding ones established in the previous work [42] for the random Airy ordinary differential equation, we consider the random fractional IVP (6.1) with $\beta=1, B \sim B e(2,3)$ $(B e(2,3)$ stands for a Beta distribution with parameters 2 and 3$)$ and the initial conditions $A_{0}$ and $A_{1}$, that are $R V$ s such that

$$
\mathbb{E}\left[A_{0}\right]=1, \mathbb{E}\left[A_{0}^{2}\right]=2, \mathbb{E}\left[A_{1}\right]=2, \mathbb{E}\left[A_{1}^{2}\right]=5 .
$$

In Tables 6.1 and 6.2, we collect the values of the approximations of the mean and variance of the solution SP computed via (6.15) and (6.17) with $M=10$, respectively. For ease of comparison, the values of the inputs as well as the time 
instants where the mean and the variance of the solution have been computed, are the same as in Example 12 of [42]. In Tables 6.1 and 6.2 the values of $\alpha$ parameter are taken numbers such as $\alpha \rightarrow 2^{-}$. We evince rapid convergence for both the mean and the variance.

\begin{tabular}{l|cccccc}
\hline \hline Mean & $\alpha=1.8$ & $\alpha=1.85$ & $\alpha=1.9$ & $\alpha=1.95$ & $\alpha=1.99$ & $\alpha=1.999$ \\
\hline \hline$t=0.00$ & 1.0000 & 1.0000 & 1.00000 & 1.0000 & 1.0000 & 1.0000 \\
\hline$t=0.25$ & 1.4978 & 1.4981 & 1.4983 & 1.4985 & 1.4987 & 1.4987 \\
\hline$t=0.50$ & 1.9814 & 1.9831 & 1.9847 & 1.9862 & 1.9873 & 1.9875 \\
\hline$t=0.75$ & 2.4325 & 2.4377 & 2.4425 & 2.4469 & 2.4503 & 2.451 \\
\hline$t=1.00$ & 2.8289 & 2.8396 & 2.8498 & 2.8594 & 2.8668 & 2.8684 \\
\hline$t=1.25$ & 3.1461 & 3.1643 & 3.1818 & 3.1987 & 3.2117 & 3.2146 \\
\hline$t=1.50$ & 3.3603 & 3.3868 & 3.4128 & 3.4382 & 3.4582 & 3.4626 \\
\hline$t=1.75$ & 3.4513 & 3.4853 & 3.5192 & 3.5530 & 3.5799 & 3.5859 \\
\hline$t=2.00$ & 3.4064 & 3.4446 & 3.4835 & 3.5231 & 3.5553 & 3.5625 \\
\hline
\end{tabular}

Table 6.1: Values of the expectation of the solution SP of the random fractional IVP (6.1) computed by (6.15) with $M=10$ in the context of Example 6.1.

\begin{tabular}{c|cccccc}
\hline \hline Variance & $\alpha=1.8$ & $\alpha=1.85$ & $\alpha=1.9$ & $\alpha=1.95$ & $\alpha=1.99$ & $\alpha=1.999$ \\
\hline \hline$t=0.00$ & 1.0000 & 1.0000 & 1.0000 & 1.0000 & 1.0000 & 1.0000 \\
\hline$t=0.25$ & 1.0589 & 1.0593 & 1.0597 & 1.0601 & 1.0603 & 1.0603 \\
\hline$t=0.50$ & 1.2227 & 1.2252 & 1.2274 & 1.2295 & 1.2311 & 1.2314 \\
\hline$t=0.75$ & 1.4680 & 1.4748 & 1.4811 & 1.4870 & 1.4915 & 1.4925 \\
\hline$t=1.00$ & 1.7639 & 1.7772 & 1.7900 & 1.8022 & 1.8116 & 1.8137 \\
\hline$t=1.25$ & 2.0769 & 2.0980 & 2.1187 & 2.1389 & 2.1548 & 2.1583 \\
\hline$t=1.50$ & 2.3820 & 2.4101 & 2.4381 & 2.4660 & 2.4882 & 2.4932 \\
\hline$t=1.75$ & 2.6755 & 2.7074 & 2.7398 & 2.7725 & 2.7990 & 2.8049 \\
\hline$t=2.00$ & 2.9803 & 3.0135 & 3.0470 & 3.0809 & 3.1084 & 3.1146 \\
\hline
\end{tabular}

Table 6.2: Values of the variance of the solution SP to the random fractional IVP (6.1) computed by (6.17) with $M=10$ in the context of Example 6.1.

To complete our numerical experiment, in Figure 6.1 we have plotted the approximations of the mean (left) and the variance (right) for different order of truncations $M$ over the time interval $0 \leq t \leq 5$. From both plots, we can visualize the convergence of both statistical moments as $M$ increases over the whole time domain in agreement with our theoretical results. Notice that we have used greater values of $M$ for the variance $(M=7,8, \ldots, 11)$ than for the mean $(M=4,5, \ldots, 8)$ to 
Chapter 6. Solving high order random non-autonomous linear fractional differential equations: generalizing Airy differential equation

get good approximations. This is an expected fact since the variance is a higher statistical moment than the mean.
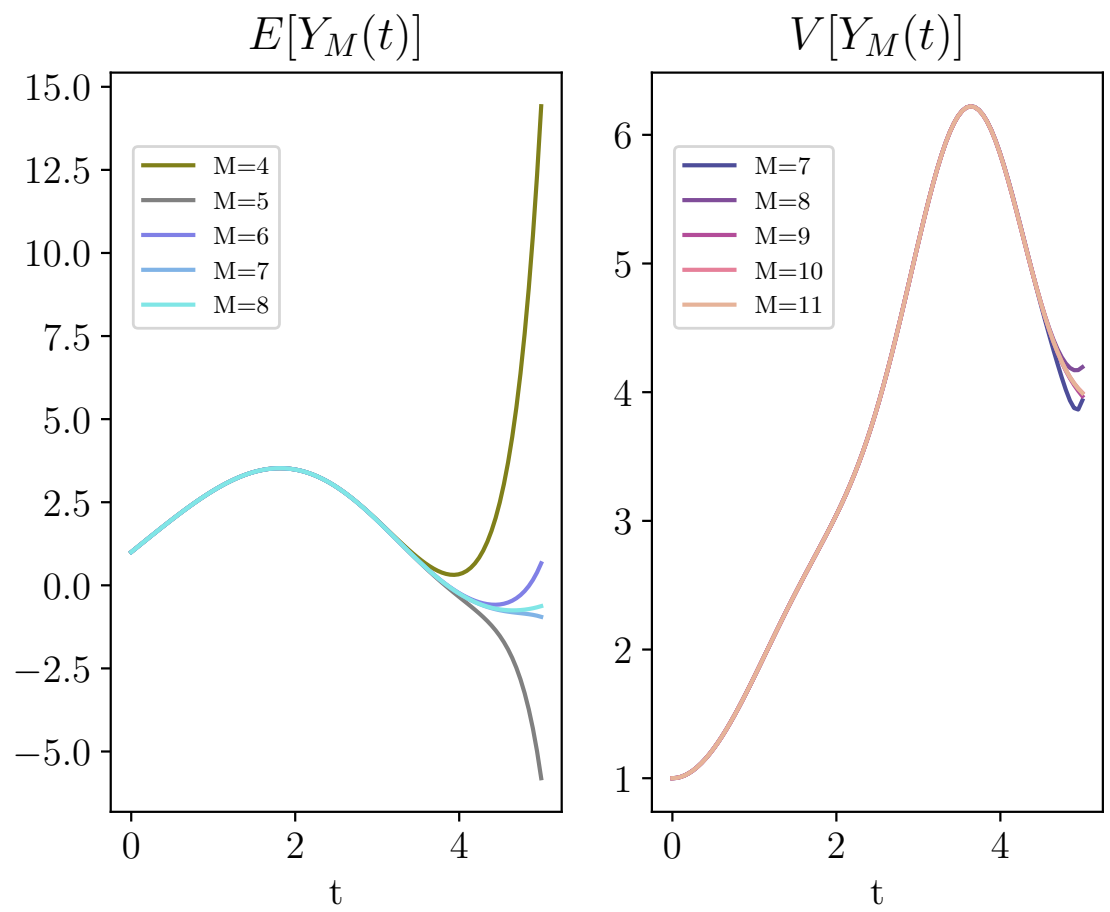

Figure 6.1: Approximations of the mean $\left(\mathbb{E}\left[Y_{M}(t)\right]\right)$ and the variance $\left(\mathbb{V}\left[Y_{M}(t)\right]\right)$ of the solution SP to the random IVP (6.1) using different orders of truncations $M$ over the interval $t \in[0,5]$ in the context of Example 6.1.

To demonstrate the validity of the approximations for larger time intervals, in Figure 6.2 we have represented the approximations of both the mean and the variance in the interval $0 \leq t \leq 9$. Notice that to compute reliable approximations on this larger time interval, greater values of the truncation order $M$ have been required. Specifically, for the mean, we have taken $M=18,19, \ldots, 22$, and for the variance, $M=21,22, \ldots, 25$. In Tables 6.3 and 6.4 we show the relative errors for the approximations of the mean and the variance, respectively, at the time instants $t=1, \ldots, 9$. As expected, we can observe that for $t$ fixed, these approximations improve as $M$ increases. 


\begin{tabular}{c|ccccccccc}
\hline \hline $\mathrm{RE}($ Mean) $(t ; M)$ & $t=1$ & $t=2$ & $t=3$ & $t=4$ & $t=5$ & $t=6$ & $t=7$ & $t=8$ & $t=9$ \\
\hline \hline$M=18$ & 0.0 & 0.0 & 0.0 & 0.0 & 0.0 & 0.0 & 0.0385 & 2.0194 & 1.2306 \\
\hline$M=19$ & 0.0 & 0.0 & 0.0 & 0.0 & 0.0 & 0.0 & 0.0041 & 0.3200 & 1.2122 \\
\hline$M=20$ & 0.0 & 0.0 & 0.0 & 0.0 & 0.0 & 0.0 & 0.0004 & 0.0697 & 1.1899 \\
\hline$M=21$ & 0.0 & 0.0 & 0.0 & 0.0 & 0.0 & 0.0 & 0.0 & 0.0089 & 1.2018 \\
\hline$M=22$ & 0.0 & 0.0 & 0.0 & 0.0 & 0.0 & 0.0 & 0.0 & 0.0011 & 1.0551 \\
\hline$M=23$ & 0.0 & 0.0 & 0.0 & 0.0 & 0.0 & 0.0 & 0.0 & 0.0001 & 3.1418 \\
\hline$M=24$ & 0.0 & 0.0 & 0.0 & 0.0 & 0.0 & 0.0 & 0.0 & 0.0 & 0.2239 \\
\hline$M=25$ & 0.0 & 0.0 & 0.0 & 0.0 & 0.0 & 0.0 & 0.0 & 0.0 & 0.0410 \\
\hline
\end{tabular}

Table 6.3: Relative error for the mean, given by (6.25), at different time instants for different orders of truncation $M$ in the context of Example 6.1.

\begin{tabular}{c|ccccccccc}
\hline \hline RE(Variance $)(t ; M)$ & $t=1$ & $t=2$ & $t=3$ & $t=4$ & $t=5$ & $t=6$ & $t=7$ & $t=8$ & $t=9$ \\
\hline \hline$M=18$ & 0.0 & 0.0 & 0.0 & 0.0 & 0.0 & 0.0 & 0.0 & 0.1519 & 0.9392 \\
\hline$M=19$ & 0.0 & 0.0 & 0.0 & 0.0 & 0.0 & 0.0 & 0.0 & 0.0002 & 0.9494 \\
\hline$M=20$ & 0.0 & 0.0 & 0.0 & 0.0 & 0.0 & 0.0 & 0.0 & 0.0007 & 0.9533 \\
\hline$M=21$ & 0.0 & 0.0 & 0.0 & 0.0 & 0.0 & 0.0 & 0.0 & 0.0001 & 0.8936 \\
\hline$M=22$ & 0.0 & 0.0 & 0.0 & 0.0 & 0.0 & 0.0 & 0.0 & 0.0 & 0.2753 \\
\hline$M=23$ & 0.0 & 0.0 & 0.0 & 0.0 & 0.0 & 0.0 & 0.0 & 0.0 & 0.0180 \\
\hline$M=24$ & 0.0 & 0.0 & 0.0 & 0.0 & 0.0 & 0.0 & 0.0 & 0.0 & 0.0006 \\
\hline$M=25$ & 0.0 & 0.0 & 0.0 & 0.0 & 0.0 & 0.0 & 0.0 & 0.0 & 0.0002 \\
\hline
\end{tabular}

Table 6.4: Relative error for the variance, given by (6.26), at different time instants for different orders of truncation $M$ in the context of Example 6.1. 

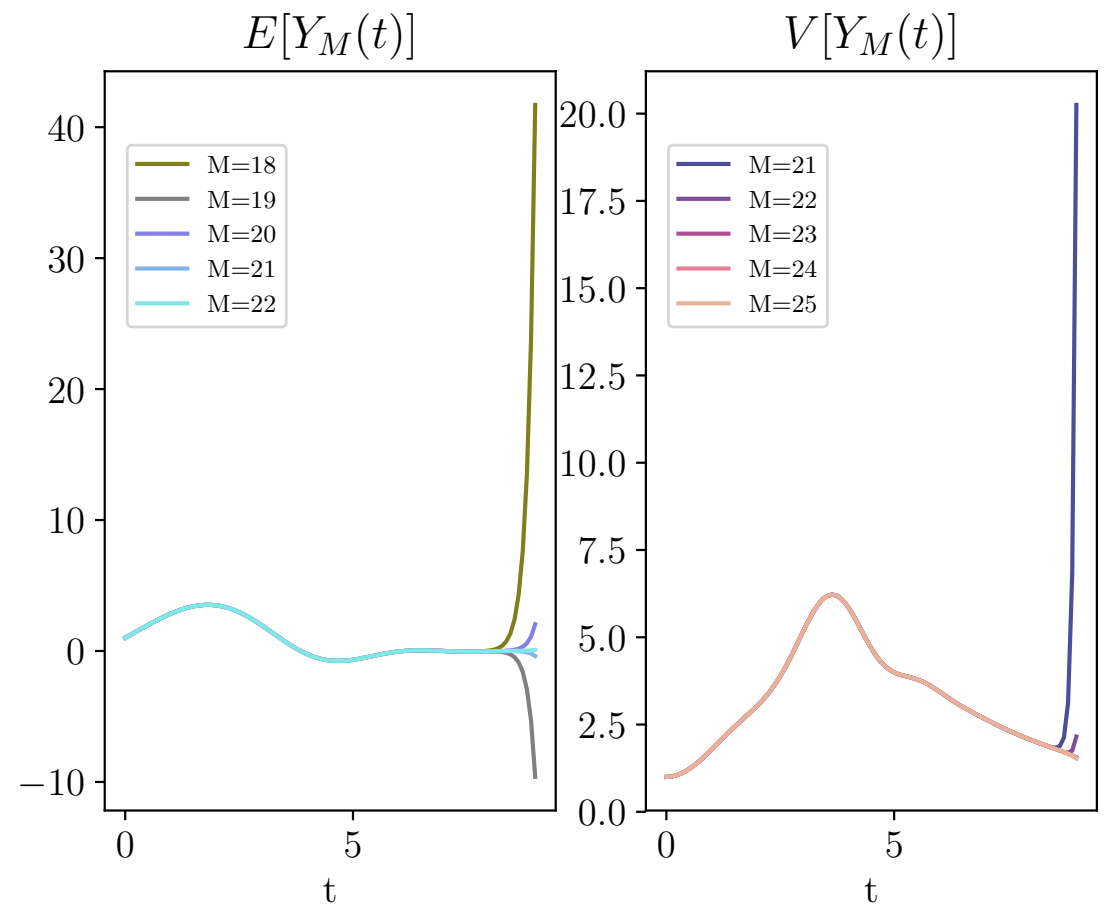

Figure 6.2: Approximations of the mean $\left(\mathbb{E}\left[Y_{M}(t)\right]\right)$ and the variance $\left(\mathbb{V}\left[Y_{M}(t)\right]\right)$ of the solution SP to the random IVP (6.1) using different orders of truncations $M$ over the interval $t \in[0,9]$ in the context of Example 6.1. 
Once the approximations for the mean and for the variance have been obtained, we proceed to compute the approximations for $f_{Y_{M}(t)}(y, t)$ that approximates the 1-PDF $f_{Y(t)}(y, t)$ via PME described in Section 6.4. To this end, we have fixed $t \in[0,9], M=30$ and $\alpha=1.9$. Using expressions (6.15) and (6.17), we have computed the moments $\mathbb{E}\left[Y_{M}(t)\right]$ and $\mathbb{E}\left[\left(Y_{M}(t)\right)^{2}\right]=\mathbb{V}\left[Y_{M}(t)\right]+\left(\mathbb{E}\left[Y_{M}(t)\right]\right)^{2}$. As we have pointed out in Section 6.4, these values are good approximations of $m_{1}(t) \equiv m_{1}$ and $m_{2}(t) \equiv m_{2}$, respectively, which are required to set the system of non-linear equations (6.24). Although the values $a_{1}$ and $a_{2}$ of the support are obviously different for each $t$, to simplify our exposition we must say that, according to Bienaymé-Chebyshev's inequality, is enough to take

$$
\left[a_{1}, a_{2}\right]=\left[\min _{t \in[0,9]} \mathbb{E}\left[Y_{M}(t)\right]-10 \sqrt{\mathbb{V}\left[Y_{M}(t)\right]}, \max _{t \in[0,9]} \mathbb{E}\left[Y_{M}(t)\right]+10 \sqrt{\mathbb{V}\left[Y_{M}(t)\right]}\right],
$$

for $M$ fixed (remember that in our case we have taken $M=30$ ). This leads to the following support $\left[a_{1}, a_{2}\right]=[-72,110]$. In order to calculate the PDF $f_{Y_{M}(t)}(y, t)$ for $t \in[0,9]$, we have taken the time instants: $t=t_{k}=0.090 k$, $k=0,1, \ldots, 100$, and we have numerically computed the solutions $\left(\lambda_{0}, \lambda_{1}, \lambda_{2}\right)$ of the non-linear system of equations (6.24). In Table 6.5, we show the values of $\lambda_{0}, \lambda_{1}, \lambda_{2}$ as well as of the moments $m_{1}$ and $m_{2}$ for $t=0,1, \ldots, 9$. To illustrate the usefulness of knowing the approximations of PDF $f_{Y_{M}(t)}(y, t)$, in the last column we have calculated the probability that the solution SP lies in the interval $\left[y_{1}, y_{2}\right]$ for $t \in[0,9]$, by applying (4.2) with $\left.y_{1}=\mathbb{E}\left[Y_{M}(t)\right]-\sqrt{\mathbb{V}\left[Y_{M}(t)\right]}\right]$ and $\left.y_{2}=\mathbb{E}\left[Y_{M}(t)\right]+\sqrt{\mathbb{V}\left[Y_{M}(t)\right]}\right]$. Finally, in Figure 6.3 we have represented the PDF $f_{Y_{M}(t)}(y, t)$ in the time interval $t \in[0,9]$ for $M=30$, and we have highlighted on the its surface the curves of the PDF corresponding to the time instants $t=0,1, \ldots, 9$.

Example 6.2 In this example we illustrate the case where $p<\alpha$. To this end, we assume that coefficient $B$ in the random IVP (6.1) has an exponential distribution with mean 4, i.e. $B \sim \operatorname{Exp}(4)$, thus $p=1$ (see Table 3.1) and $\alpha=2.6$. We will take $\beta=1.7$ and the two first statistical moments w.r.t. the origin of the three random initial conditions are assumed to be

$$
\mathbb{E}\left[A_{0}\right]=1, \mathbb{E}\left[A_{0}^{2}\right]=2, \mathbb{E}\left[A_{1}\right]=2, \mathbb{E}\left[A_{1}^{2}\right]=5, \mathbb{E}\left[A_{2}\right]=4, \mathbb{E}\left[A_{2}^{2}\right]=20 .
$$


Chapter 6. Solving high order random non-autonomous linear fractional differential equations: generalizing Airy differential equation

\begin{tabular}{c|cccccc}
\hline \hline & $\lambda_{0}$ & $\lambda_{1}$ & $\lambda_{2}$ & $m_{1}=\mathbb{E}\left[Y_{30}(t)\right]$ & $m_{2}=\mathbb{E}\left[\left(Y_{30}(t)\right)^{2}\right]$ & $\mathbb{P}\left[y_{1} \leq Y(t) \leq y_{2}\right]$ \\
\hline \hline$t=0$ & 2.4189 & -1.0000 & 0.5000 & 1 & 2 & 0.686141 \\
\hline$t=1$ & 4.5155 & -1.5000 & 0.2500 & 3 & 11 & 0.684482 \\
\hline$t=2$ & 5.2236 & -1.0000 & 0.1000 & 5 & 30 & 0.684439 \\
\hline$t=3$ & 5.5202 & -0.7000 & 0.0500 & 7 & 59 & 0.683465 \\
\hline$t=4$ & 5.7179 & -0.5294 & 0.0290 & 9 & 98 & 0.683692 \\
\hline$t=5$ & 5.8749 & -0.4230 & 0.0190 & 11 & 206 & 0.681471 \\
\hline$t=6$ & 6.0082 & -0.3513 & 0.0135 & 13 & 275 & 0.683482 \\
\hline$t=7$ & 6.1250 & -0.3000 & 0.0100 & 15 & 354 & 0.681515 \\
\hline$t=8$ & 6.2292 & -0.2615 & 0.0076 & 17 & 443 & 0.679679 \\
\hline$t=9$ & 6.3235 & -0.2317 & 0.0060 & 19 & 0.682466
\end{tabular}

Table 6.5: Values of $\lambda_{0}, \lambda_{1}, \lambda_{2}$ that solve the non-linear system of equations (6.24) (with $m_{1}$ and $m_{2}$ given in the corresponding columns) and determine the PDF $g(y)$ (and thus $\left.f_{Y_{M}(t)}(y, t)\right)$ given by $(6.22)$ for $M=30$ at different times instants $t=0,1,2, \ldots, 9$. This function approximates the $\mathrm{PDF} f_{Y(t)}(y, t)$ of the solution SP to random fractional IVP (6.1) for the order of the fractional derivative $\alpha=1.9$ in the context of Example 6.1. In the last column we give the probability that the solution lies in the interval $\left[y_{1}, y_{2}\right]$ being $\left.y_{1}=\mathbb{E}\left[Y_{M}(t)\right]-\sqrt{\mathbb{V}\left[Y_{M}(t)\right]}\right]$ and $\left.y_{2}=\mathbb{E}\left[Y_{M}(t)\right]+\sqrt{\mathbb{V}\left[Y_{M}(t)\right]}\right]$.

$$
f_{Y_{30}}(y ; t)
$$

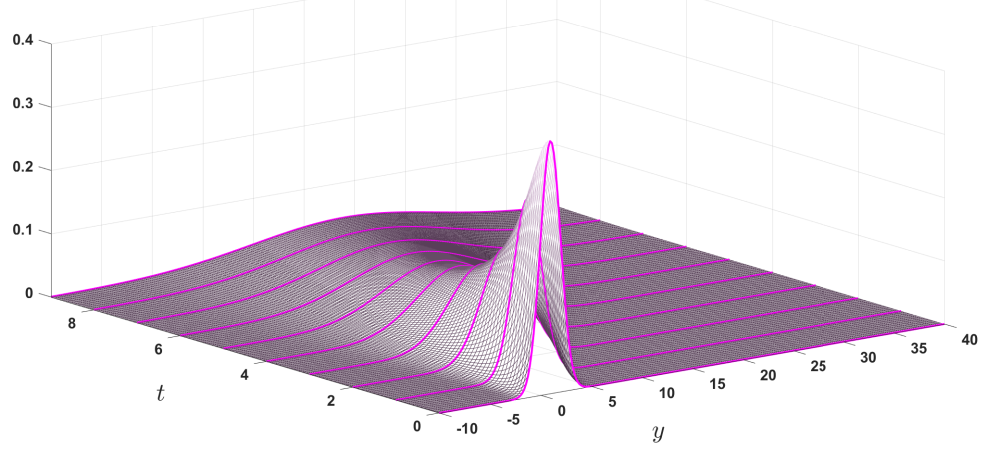

Figure 6.3: Approximate PDF, $f_{Y_{M}(t)}(y, t)$, of the solution SP $Y(t)$ to random fractional IVP (6.1) with order of the fractional order $\alpha=1.9$ in the context of Example 6.1 using the $\mathrm{PME}$ for the approximate solution SP $Y_{M}(t)$ with $M=30$ and $0 \leq t \leq 9$. 


\begin{tabular}{c|ccccccc}
\hline \hline RE(Mean) $(t ; M)$ & $t=1$ & $t=2$ & $t=3$ & $t=4$ & $t=5$ & $t=6$ & $t=7$ \\
\hline \hline$M=2$ & 0.0 & 0.0 & 0.0044 & 0.0781 & 0.4369 & 1.3189 & 2.9417 \\
\hline$M=3$ & 0.0 & 0.0 & 0.0003 & 0.0155 & 0.1674 & 0.6796 & 1.7185 \\
\hline$M=4$ & 0.0 & 0.0 & 0.0 & 0.0024 & 0.0569 & 0.3487 & 1.0506 \\
\hline$M=5$ & 0.0 & 0.0 & 0.0 & 0.0003 & 0.0163 & 0.1698 & 0.6493 \\
\hline$M=6$ & 0.0 & 0.0 & 0.0 & 0.0 & 0.0038 & 0.0756 & 0.3956 \\
\hline$M=7$ & 0.0 & 0.0 & 0.0 & 0.0 & 0.0007 & 0.0299 & 0.2326 \\
\hline$M=8$ & 0.0 & 0.0 & 0.0 & 0.0 & 0.0001 & 0.0103 & 0.1293 \\
\hline$M=9$ & 0.0 & 0.0 & 0.0 & 0.0 & 0.0 & 0.0031 & 0.0667 \\
\hline
\end{tabular}

Table 6.6: Relative error for the mean, given by (6.25), at different time instants for different orders of truncation $M$ in the context of Example 6.2.

\begin{tabular}{c|ccccccc}
\hline \hline $\mathrm{RE}($ Variance $)(t ; M)$ & $t=1$ & $t=2$ & $t=3$ & $t=4$ & $t=5$ & $t=6$ & $t=7$ \\
\hline \hline$M=2$ & 0.0 & 0.0002 & 0.0427 & 0.6461 & 3.6097 & 14.6488 & 49.8305 \\
\hline$M=3$ & 0.0 & 0.0 & 0.0037 & 0.1628 & 1.3583 & 6.1317 & 21.3876 \\
\hline$M=4$ & 0.0 & 0.0 & 0.0002 & 0.035 & 0.5557 & 3.0331 & 11.0941 \\
\hline$M=5$ & 0.0 & 0.0 & 0.0 & 0.0059 & 0.2195 & 1.6144 & 6.3768 \\
\hline$M=6$ & 0.0 & 0.0 & 0.0 & 0.0008 & 0.0775 & 0.8774 & 3.8925 \\
\hline$M=7$ & 0.0 & 0.0 & 0.0 & 0.0001 & 0.0232 & 0.4687 & 2.46 \\
\hline$M=8$ & 0.0 & 0.0 & 0.0 & 0.0 & 0.0058 & 0.2379 & 1.5805 \\
\hline$M=9$ & 0.0 & 0.0 & 0.0 & 0.0 & 0.0012 & 0.1113 & 1.0168 \\
\hline
\end{tabular}

Table 6.7: Relative error for the variance, given by (6.26), at different time instants for different orders of truncation $M$ in the context of Example 6.2. 
Chapter 6. Solving high order random non-autonomous linear fractional differential equations: generalizing Airy differential equation

In Figure 6.4, we show the approximations of the mean, $\mathbb{E}\left[Y_{M}(t)\right]$, and the standard deviation, $\sigma\left[Y_{M}(t)\right]$, for different orders of truncations $M=2,3, \ldots, 6$ in the interval $t \in[0,5]$. We observe convergence being faster for the mean than for the standard deviation, as expected. According to Theorem 6.1 and Proposition 2.10, in this context convergence of the mean and the standard deviation (or equivalently, the variance) takes place for every $t$. Naturally, to get better approximations the order of truncation $M$ needs to be increased as $t$ departs from the origin, $t=0$, where the initial condition is set. To better assess the convergence, in Tables 6.6 and 6.7 we have collected the values for the relative errors of the mean and the variance. We can see that figures are in fully agreement with the previous comments.

To complete the graphical analysis in this example, in Figure 6.5 we show the surface of the correlation coefficient

$$
\rho_{Y_{M}}(t, s)=\frac{\mathbb{C}_{Y_{M}, Y_{M}}(t, s)}{\sigma_{Y_{M}}(t) \sigma_{Y_{M}}(s)},
$$

for $M=6$ (where numerical results are stabilized) over the domain $(t, s) \in$ $[0,5] \times[0,5]$. Observe that the coefficient of correlation takes its highest value, namely 1, when $t=s$ (on the diagonal of the domain) since in this case $Y_{M}(t)$ and $Y_{M}(s)$ are completely and positively correlated since both match. From this graphical representation and the statistical interpretation of the correlation coefficient, we observe that the linear dependence between $R V s Y_{M}(t)$ and $Y_{M}(s)$ decreases to 0 (uncorrelated) when $t \rightarrow 5$ and $s \rightarrow 0$ or vice versa, $t \rightarrow 0$ and $s \rightarrow 5$.

Example 6.3 This example is devised to illustrate the case the m.s. convergence of the solution stochastic process (6.12)-(6.13) (hence its mean and its variance too) on a bounded interval, that is, when $p=\alpha$ (see Theorem 6.1 and Proposition 2.10). In such a case, the interval of convergence is given by $\mathcal{D}=\left[0, \frac{(\alpha+\beta)^{\frac{\alpha}{\alpha+\beta}}}{\mathcal{H}^{\frac{1}{\alpha+\beta}}}[\right.$ (see Theorem 6.1). To this end, we choose $\alpha=4.2$ and $B$ is a Weibull $R V$ of parameters $a=0.001$ and $b=1 / \alpha$ (see Table 3.1). So, according to Table 3.1, $\mathcal{H}=a(2 / b)^{1 / b}=7.62$. As a consequence $\mathcal{D}=[0,2.56271[$. For the rest of input parameters of the random IVP (6.1), we take $\beta=1$ and

$$
\mathbb{E}\left[A_{0}\right]=0, \mathbb{E}\left[A_{0}^{2}\right]=2, \mathbb{E}\left[A_{1}\right]=1, \mathbb{E}\left[A_{1}^{2}\right]=5, \mathbb{E}\left[A_{2}\right]=4, \mathbb{E}\left[A_{2}^{2}\right]=17 .
$$



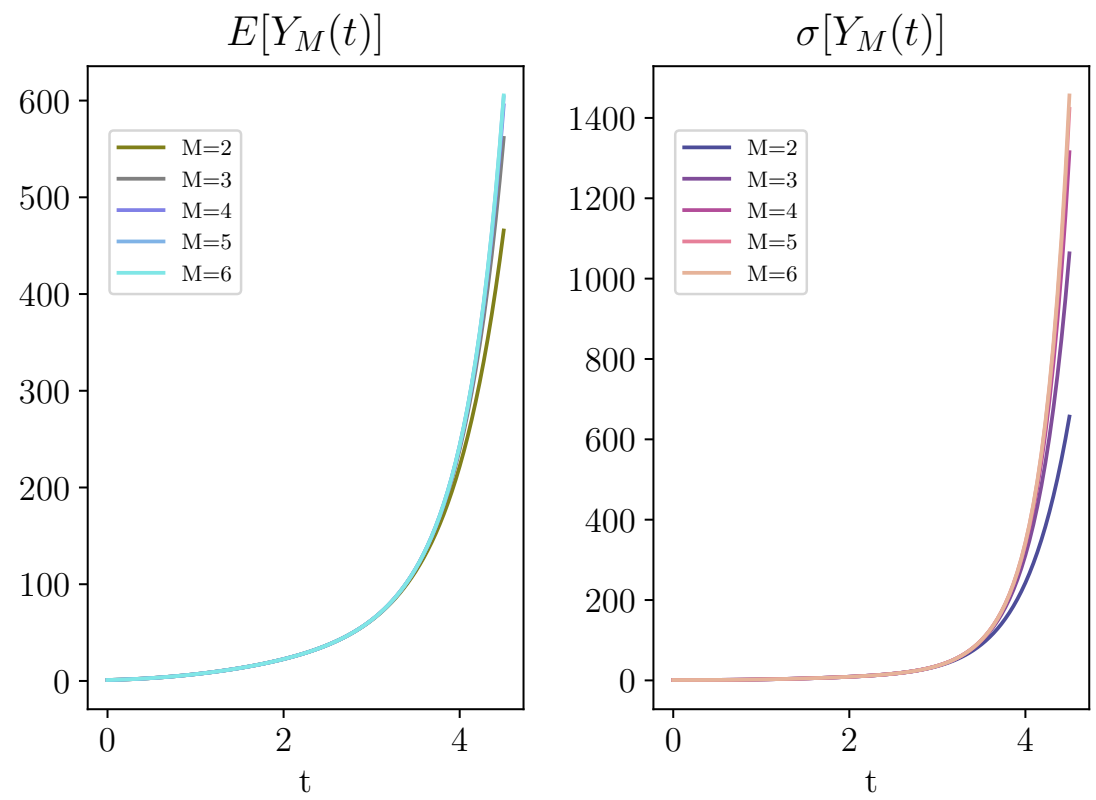

Figure 6.4: Approximations of the mean $\left(\mathbb{E}\left[Y_{M}(t)\right]\right)$ and the standard deviation $\left(\sigma\left[Y_{M}(t)\right]\right)$ of the solution SP to the random IVP (6.1) using different orders of truncations $M$ in the interval $t \in[0,5]$ in the context of Example 6.2. 


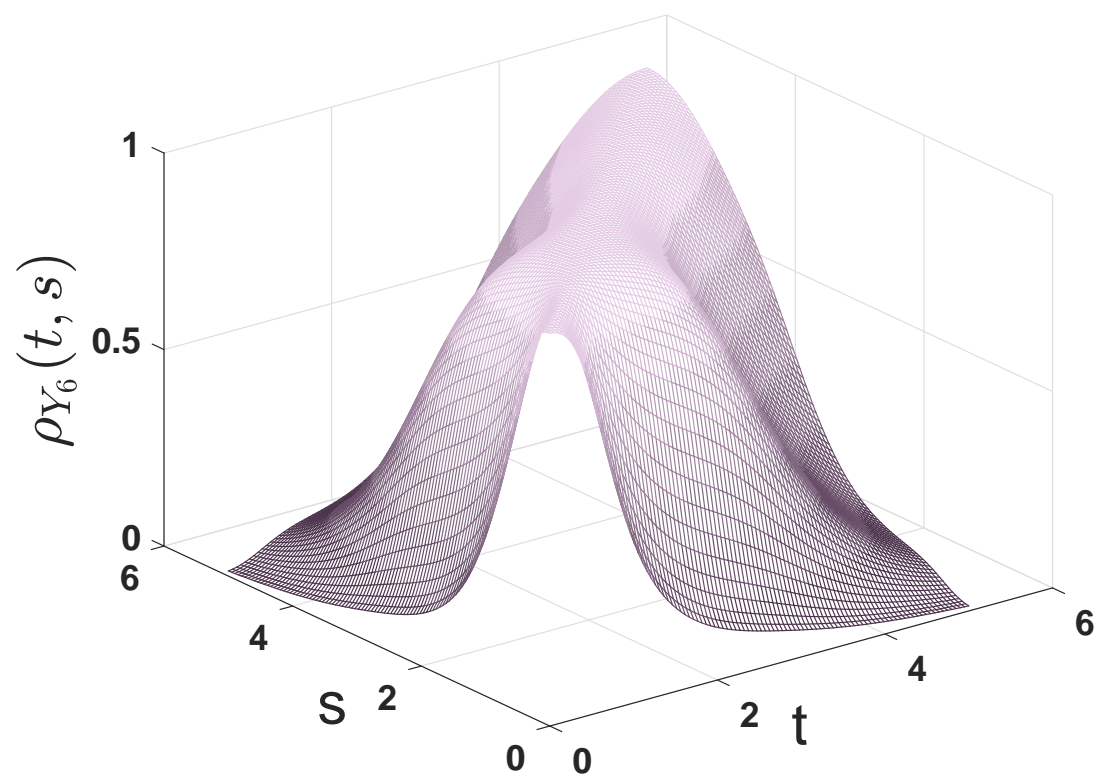

Figure 6.5: Surface of the correlation coefficient of the solution SP, defined in (6.27), with $M=6$ over the domain $(t, s) \in[0,5] \times[0,5]$ in the context of Example 6.2. 


\begin{tabular}{c|ccc|cccc}
\hline \hline RE(Mean) $(t ; M)$ & $t=1$ & $t=2$ & $t=3$ & $t=2.4$ & $t=2.5$ & $t=2.6$ & $t=2.7$ \\
\hline \hline$M=2$ & 0.0 & 0.00119 & 0.10665 & 0.01083 & 0.01704 & 0.02598 & 0.03844 \\
\hline$M=3$ & 0.0 & $3 e-05$ & 0.02084 & 0.00069 & 0.00134 & 0.00252 & 0.00452 \\
\hline$M=4$ & 0.0 & 0.0 & 0.00372 & $4 e-05$ & $9 e-05$ & 0.00021 & 0.00046 \\
\hline$M=5$ & 0.0 & 0.0 & 0.0006 & 0.0 & $1 e-05$ & $2 e-05$ & $4 e-05$ \\
\hline$M=6$ & 0.0 & 0.0 & $9 e-05$ & 0.0 & 0.0 & 0.0 & 0.0 \\
\hline$M=7$ & 0.0 & 0.0 & $1 e-05$ & 0.0 & 0.0 & 0.0 & 0.0 \\
\hline$M=8$ & 0.0 & 0.0 & 0.0 & 0.0 & 0.0 & 0.0 & 0.0 \\
\hline$M=9$ & 0.0 & 0.0 & 0.0 & 0.0 & 0.0 & 0.0 & 0.0 \\
\hline
\end{tabular}

Table 6.8: Relative error for the mean, given by (6.25), at different time instants for different orders of truncation $M$ in the context of Example 6.3.

\begin{tabular}{c|ccc|cccc}
\hline \hline $\mathrm{RE}($ Variance $)(t ; M)$ & $t=1$ & $t=2$ & $t=3$ & $t=2.4$ & $t=2.5$ & $t=2.6$ & $t=2.7$ \\
\hline \hline$M=2$ & 0.00018 & 0.80822 & 37.35409 & 4.61293 & 6.73642 & 9.6965 & 13.78835 \\
\hline$M=3$ & 0.0 & 0.27955 & 22.74314 & 2.45614 & 3.75086 & 5.57471 & 8.10991 \\
\hline$M=4$ & 0.0 & 0.10046 & 17.53194 & 1.6142 & 2.6038 & 4.01809 & 6.00033 \\
\hline$M=5$ & 0.0 & 0.03358 & 14.74921 & 1.15213 & 1.97189 & 3.16210 & 4.84565 \\
\hline$M=6$ & 0.0 & 0.01006 & 12.90896 & 0.85548 & 1.55995 & 2.59958 & 4.08308 \\
\hline$M=7$ & 0.0 & 0.0027 & 11.56632 & 0.64987 & 1.26903 & 2.19841 & 3.5352 \\
\hline$M=8$ & 0.0 & 0.00065 & 10.54187 & 0.50095 & 1.05405 & 1.89955 & 3.12451 \\
\hline$M=9$ & 0.0 & 0.00015 & 9.73999 & 0.38981 & 0.89008 & 1.67022 & 2.80801 \\
\hline
\end{tabular}

Table 6.9: Relative error for the variance, given by (6.26), at different time instants for different orders of truncation $M$ in the context of Example 6.3.

$$
\mathbb{E}\left[A_{3}\right]=0.5, \mathbb{E}\left[A_{3}^{2}\right]=0.26, \mathbb{E}\left[A_{4}\right]=0.75, \mathbb{E}\left[A_{4}^{2}\right]=0.57
$$

In order to check that convergence of the mean and the variance in the interval $\mathcal{D}=\left[0, \frac{(\alpha+\beta)^{\frac{\alpha}{\alpha+\beta}}}{\mathcal{H}^{\frac{1}{\alpha+\beta}}}[\right.$, in Tables 6.8 and 6.9 , we have computed the relative errors of consecutive approximations of both statistical moments (see expressions (6.25) and (6.26)), respectively. From the figures shown in these tables we numerically evince convergence as $M$ increases for $t \in \mathcal{D}$. To highlight the numerical behaviour of these relative errors about the value upper of the convergence interval, i.e. $t=2.56271$, we have included in Table 6.8 and Table 6.9 the values corresponding to times $t=2.4,2.5,2.6,2.7$. Observe that relative errors also decrease beyond $t=2.56271$ illustrating the comments raised in Remark 6.2. 


\subsection{Conclusions}

In this chapter we have studied an important class of RFDEs, given by (6.1), that include as particular case the random Airy differential equation. We have constructed approximations of the solution SP by means of random generalized power series and we have established sufficient conditions on random inputs (initial conditions and the coefficient $B$ ) in order to guarantee that random series is m.s. convergent. The use of m.s. convergence in our analysis is a key point since from its properties the convergence of the mean and the variance of the approximations to the corresponding exact ones are guaranteed. Furthermore, we have given explicit expressions to construct reliable approximations to the mean, the variance and the covariance functions of the solution. The study has relied heavily on the assumption made about the growth of statistical moments of the input coefficient $B$. We have shown that a wide range of important RVs satisfy such hypothesis. In future research, we will investigate alternative assumptions to $B$ in order to broaden the family of unbounded RVs that can play the role of input $B$. Nevertheless, an important issue of this chapter is that we can approximate accurately unbounded RVs by truncating its domain so that the involved probabilistic error be as small as desired to get good approximations taking advantage of the results established here. Furthermore, we have taken advantage of the approximations of the mean and the variance of the solution SP for the random fractional generalized Airy differential equation to construct reliable approximations of the probability density function of the solution. This is an important application of our approach since from the density function one can obtain a more comprehensive description of the solution via all its one-dimensional statistical moments and permits computing the probability that the solution lies in intervals of interest. Finally, numerical experiments have been developed in order to illustrate the theoretical findings. They evince the proposed method provides good approximations for the mean and the variance of the solution using a small order of truncation. 


\section{Chapter published}

The results of this chapter have been published in [22]. With regard to this paper, the $\mathrm{PhD}$ candidate has contributed by working in its complete development with more emphasis on obtaining a general convergent solution and its main statistical moments, constructing approximations of the 1-PDF using PME, devising the numerical examples and participating in writing the paper. 



\section{Chapter}

\section{Mean square convergent numerical solutions of random fractional differential equations: Approximations of moments and density}

In the previous chapters exact solutions for random fractional initial value problems have been obtained. Nevertheless, it is not always possible and numerical methods are required to obtain approximations for the solution of random fractional initial value problems. In this chapter, a fractional forward Euler-like method is developed to solve random fractional initial value problems via Caputo derivative. The analysis is conducted by using the so-called random mean square calculus. Under mild conditions on the input data, the mean square convergence of the numerical scheme is proved. This type of stochastic convergence guarantees the approximations of the mean and the variance of the solution stochastic process, computed via the aforementioned numerical scheme, will converge to their corresponding exact values. The theoretical analysis is illustrated by two examples where approximations of the 1-PDF are also computed combining the approximations for the two main statistical moments and the Principle of Maximum Entropy described in Section 6.4. 


\subsection{Introduccion}

In this chapter, we deal with random fractional IVPs of the form

$$
\left\{\begin{array}{cl}
\left({ }^{C} D_{a^{+}}^{\alpha} X\right)(t) & =f(X(t), t), \quad t \in[a, b], \quad 0<\alpha \leq 1, \\
X(a) & =X_{0},
\end{array}\right.
$$

where $\left({ }^{C} D_{a^{+}}^{\alpha} X\right)(t)$ is the random m.s. Caputo fractional derivative defined in (2.16). Throughout this chapter, the initial condition, $X_{0}$ is assumed to be a $2-\mathrm{RV}$, i.e., $X_{0} \in \mathrm{L}^{2}(\Omega)$. Additionally, we will assume that the function $f$ defining the right-hand side of fractional differential equation (7.1), i.e. $f: S \times[a, b] \rightarrow \mathrm{L}^{2}(\Omega)$, $S \subseteq \mathrm{L}^{2}(\Omega)$, satisfies the following conditions:

N1: $f$ is m.s. Lipschitz, that is, there exists $\kappa>0$ such that

$$
\|f(X, t)-f(Y, t)\|_{2} \leq \kappa\|X-Y\|_{2}, \quad X, Y \in \mathrm{L}^{2}(\Omega) .
$$

N2: Given a bounded RV $S, f$ satisfies the m.s. modulus of continuity property, i.e.,

$$
\lim _{h \rightarrow 0} W(S, h)=0 \quad W(S, h)=\sup _{X \in S \subseteq \mathrm{L}^{2}(\Omega)} \sup _{\left|t-t^{\prime}\right| \leq|h|}\left\|f(X, t)-f\left(X, t^{\prime}\right)\right\|_{2} .
$$

The chapter is organized as follows. Section 7.2 addresses the relation between the IVP (7.1) and a random Volterra integral equation. Sections 7.3 and 7.4 are devoted to formulate the random fractional forward Euler-like numerical scheme and to prove its m.s. convergence, respectively. In Section 7.5, we give explicit approximations of the mean, the variance and the covariance obtained by the random numerical scheme. Section 7.6 presents several illustrative examples. Conclusions are drawn in last section. 


\subsection{Relation between the random fractional differential and inte- gral equations}

As it has been previously pointed out, the aim of the chapter is to construct m.s. approximations to the solution SP to the random fractional IVP given by (7.1). To this end, we will first study the m.s. solution of the following random integral equation

$$
X(t)=X_{0}+\frac{1}{\Gamma(\alpha)} \int_{a}^{t}(t-s)^{\alpha-1} f(X(s), s) \mathrm{d} s, \quad t \in[a, b], \quad 0<\alpha \leq 1,
$$

and its connection with the solution of the random fractional IVP given by (7.1). Let us remind that the random m.s. Riemann-Liouville fractional integral of $X(t)$, $J_{a^{+}}^{\alpha}: \mathrm{L}^{2}(\Omega) \rightarrow \mathrm{L}^{2}(\Omega)$ defined in $(2.13)$ is given as

$$
J_{a^{+}}^{\alpha}(X(t)):=\left\{\begin{array}{cll}
\frac{1}{\Gamma(\alpha)} \int_{a}^{t}(t-s)^{\alpha-1} X(s) \mathrm{d} s & \text { if } & 0<\alpha \leq 1 \\
X(t) & \text { if } & \alpha=0,
\end{array}\right.
$$

for each $t \in[a, b]$. From hypotheses $\mathbf{N} 1$ and $\mathbf{N 2}$, we know that $f(X(t), t)$ is m.s. continuous for $t \in[a, b]$, hence $f(X(t), t)$ is also m.s. uniformly continuous in $t \in[a, b]$, i.e, there exists $M>0$ such that $\|f(X(t), t)\|_{2} \leq M$, for $t \in[a, b]$. As a consequence, $\left\|\int_{a}^{t}(t-s)^{\alpha-1} f(X(s), s) \mathrm{d} s\right\|_{2} \leq M \int_{a}^{t}(t-s)^{\alpha-1} \mathrm{~d} s=M(t-a)^{\alpha} / \alpha$ and hence, since $X_{0} \in \mathrm{L}^{2}(\Omega)$, using the triangular inequality in (7.2), we derive that $X(t) \in \mathrm{L}^{2}(\Omega)$. Additionally, notice that from the fractional differential equation with $\alpha=1$, since $f(X(t), t)$ is m.s. continuous for $t \in[a, b]$, we deduce that $X^{\prime}(t)$ is of class $C^{1}([a, b])$ in the m.s. sense, thus $X(t)$ is m.s. uniformly continuous in $t \in[a, b]$, which guarantees the integral operator $J_{a^{+}}^{\alpha}(X(t))$ is well-defined. Using this operator and defining $Z(t):=f(X(t), t) \in \mathrm{L}^{2}(\Omega)$ for each, $t \in[a, b]$, the integral equation in $(7.2)$ reads as

$$
X(t)=X_{0}+J_{a^{+}}^{\alpha}(Z(t)), \quad t \in[a, b], \quad 0<\alpha \leq 1 .
$$


The next result establishes a relationship between the solutions of the integral equation given by (7.4) and the IVP given by (7.1). To prove it, first we need the following auxiliary result.

Lemma 7.1 Let $\alpha, \beta \geq 0$. If $Y(t)$ is m.s. continuous on $[a, b]$, then for each $t \in[a, b]$,

$$
J_{a^{+}}^{\alpha}\left(J_{a^{+}}^{\beta}(Y(t))\right)=J_{a^{+}}^{\alpha+\beta}(Y(t))
$$

Proof. If $\alpha$ or $\beta$ are zero, the identity is evident by the definition of the operator $J_{a^{+}}^{\alpha},(7.3)$. Now, we assume that $\alpha, \beta>0$. By definition of the operator $J_{a^{+}}^{\beta}$, it follows that $J_{a^{+}}^{\beta}(Y(s)):=\frac{1}{\Gamma(\beta)} \int_{a}^{s}(s-r)^{\beta-1} Y(r) \mathrm{d} r$, for each $s \in[a, b]$. Now, for each $t$ in $[a, b]$,

$$
\begin{aligned}
J_{a^{+}}^{\alpha}\left(J_{a^{+}}^{\beta}(Y(t))\right) & =\frac{1}{\Gamma(\alpha)} \int_{a}^{t}(t-s)^{\alpha-1} J_{a^{+}}^{\beta}(Y(s)) \mathrm{d} s \\
& =\frac{1}{\Gamma(\alpha)} \int_{a}^{t}(t-s)^{\alpha-1}\left(\frac{1}{\Gamma(\beta)} \int_{a}^{s}(s-r)^{\beta-1} Y(r) \mathrm{d} r\right) \mathrm{d} s \\
& =\frac{1}{\Gamma(\alpha)} \frac{1}{\Gamma(\beta)} \int_{a}^{t}\left(\int_{a}^{s}(t-s)^{\alpha-1}(s-r)^{\beta-1} Y(r) \mathrm{d} r\right) \mathrm{d} s .
\end{aligned}
$$

As for each $t \in[a, b]$ fixed, the triangle $\Delta=\{(s, r): a \leq s \leq t, a \leq r \leq s\}$ can also be written as $\Delta=\{(s, r): r \leq s \leq t, a \leq r \leq t\}$, the above double m.s. integral over $\Delta$ on the right-hand side of (7.5) is

$$
\begin{aligned}
& \frac{1}{\Gamma(\alpha)} \frac{1}{\Gamma(\beta)} \int_{a}^{t} \int_{r}^{t}(t-s)^{\alpha-1}(s-r)^{\beta-1} Y(r) \mathrm{d} s \mathrm{~d} r \\
& \quad=\frac{1}{\Gamma(\alpha)} \frac{1}{\Gamma(\beta)} \int_{a}^{t} Y(r)\left(\int_{r}^{t}(t-s)^{\alpha-1}(s-r)^{\beta-1} \mathrm{~d} s\right) \mathrm{d} r .
\end{aligned}
$$

The substitution $\tau=t-s$ yields

$$
J_{a^{+}}^{\alpha}\left(J_{a^{+}}^{\beta}(Y(t))\right)=\frac{1}{\Gamma(\alpha)} \frac{1}{\Gamma(\beta)} \int_{a}^{t} Y(r)\left(\int_{0}^{t-r} \tau^{\alpha-1}(t-\tau-r)^{\beta-1} \mathrm{~d} \tau\right) \mathrm{d} r .
$$


A second substitution $v=\frac{\tau}{t-r}$ implies

$$
J_{a^{+}}^{\alpha}\left(J_{a^{+}}^{\beta}(Y(t))\right)=\frac{1}{\Gamma(\alpha)} \frac{1}{\Gamma(\beta)} \int_{a}^{t}\left(\int_{0}^{1} v^{\alpha-1}(t-r)^{\alpha+\beta-1}(1-v)^{\beta-1} \mathrm{~d} v\right) Y(r) \mathrm{d} r
$$

Since $\int_{0}^{1} v^{\alpha-1}(1-v)^{\beta-1} \mathrm{~d} v=\frac{\Gamma(\alpha) \Gamma(\beta)}{\Gamma(\alpha+\beta)}$ one gets,

$$
J_{a^{+}}^{\alpha}\left(J_{a^{+}}^{\beta}(Y(t))\right)=\frac{1}{\Gamma(\alpha+\beta)} \int_{a}^{t}(t-r)^{\alpha+\beta-1} Y(r) \mathrm{d} r=J_{a^{+}}^{\alpha+\beta}(Y(t)) .
$$

Theorem 7.1 Let $X(t)$ be a m.s. solution of the integral equation given by (7.2), where $f(X(t), t)$ satisfies hypotheses $\mathbf{N 1}$ and $\mathbf{N 2}$. Then, $X(t)$ is a m.s. solution of the random fractional IVP (7.1).

Proof. As above, define $Z(t):=f(X(t), t)$. Hence, from the integral equation in terms of the operator $J_{a^{+}}^{\alpha}$, we derive

$$
X(t)-X_{0}=J_{a^{+}}^{\alpha}(Z(t)), \quad t \in[a, b], \quad 0<\alpha \leq 1 .
$$

As $0<\alpha \leq 1, \beta:=1-\alpha \geq 0$. By applying the operator $J_{a^{+}}^{1-\alpha}$ to both sides of the equation (7.5) and using Lemma 7.1 we obtain

$$
J_{a^{+}}^{1-\alpha}\left(X(t)-X_{0}\right)=J_{a^{+}}^{1-\alpha}\left(J_{a^{+}}^{\alpha}(Z(t))\right)=J_{a^{+}}^{1}(Z(t))
$$

Notice that, in view of hypotheses, we have used that $Z(t)$ is m.s. continuous on $[a, b]$. Furthermore, by property (5) of m.s. integrals in [109, page 103], this also entails that $J_{a^{+}}^{1}(Z(t))$ is m.s. differentiable on $(a, b)$. Hence, using (7.6) one gets

$$
\frac{\mathrm{d}}{\mathrm{d} t}\left(J_{a^{+}}^{1-\alpha}\left(X(t)-X_{0}\right)\right)=\frac{\mathrm{d}}{\mathrm{d} t}\left(J_{a^{+}}^{1-\alpha}(X(t))\right)-\frac{\mathrm{d}}{\mathrm{d} t}\left(J_{a^{+}}^{1-\alpha}\left(X_{0}\right)\right)=\frac{\mathrm{d}}{\mathrm{d} t}\left(J_{a^{+}}^{1}(Z(t))\right) .
$$

Now, we compute the m.s. derivatives $\frac{\mathrm{d}}{\mathrm{d} t}\left(J_{a^{+}}^{1-\alpha}\left(X_{0}\right)\right)$ and $\frac{\mathrm{d}}{\mathrm{d} t}\left(J_{a^{+}}^{1-\alpha}(X(t))\right)$. First, note that

$$
J_{a^{+}}^{1-\alpha}\left(X_{0}\right)=\frac{1}{\Gamma(1-\alpha)} \int_{a}^{t}(t-s)^{-\alpha} X_{0} \mathrm{~d} s=\frac{X_{0}(t-a)^{1-\alpha}}{(1-\alpha) \Gamma(1-\alpha)}
$$


SO

$$
\frac{\mathrm{d}}{\mathrm{d} t}\left(J_{a^{+}}^{1-\alpha}\left(X_{0}\right)\right)=\frac{X_{0}(t-a)^{-\alpha}}{\Gamma(1-\alpha)} .
$$

Next, $J_{a^{+}}^{1-\alpha}(X(t))=\frac{1}{\Gamma(1-\alpha)} \int_{a}^{t}(t-s)^{-\alpha} X(s) \mathrm{d} s$. Now, since $X(t)$ is m.s. integrable and $f(t, s)=(t-s)^{-\alpha}$ is almost everywhere continuous in $(t, s) \in[a, b] \times[a, b]$, the m.s. Leibniz rule yields

$$
\frac{\mathrm{d}}{\mathrm{d} t}\left(J_{a^{+}}^{1-\alpha}(X(t))\right)=\frac{1}{\Gamma(1-\alpha)} \int_{a}^{t}(-\alpha)(t-s)^{-\alpha-1} X(s) \mathrm{d} s .
$$

By using the m.s. integration by parts formula, one gets

$$
\frac{\mathrm{d}}{\mathrm{d} t}\left(J_{a^{+}}^{1-\alpha}(X(t))\right)=\frac{1}{\Gamma(1-\alpha)}\left(X(a)(t-a)^{-\alpha}+\int_{a}^{t}(t-s)^{-\alpha} X^{\prime}(s) \mathrm{d} s\right) .
$$

By definition, $\left({ }^{C} D_{a^{+}}^{\alpha} X\right)(t)=\frac{1}{\Gamma(1-\alpha)} \int_{a}^{t}(t-s)^{-\alpha} X^{\prime}(s) \mathrm{d} s$. Taking into account (7.8), (7.9) and the initial condition $X(a)=X_{0}$ of IVP (7.1), we deduce

$$
\frac{\mathrm{d}}{\mathrm{d} t}\left(J_{a^{+}}^{1-\alpha}(X(t))\right)=\frac{\mathrm{d}}{\mathrm{d} t}\left(J_{a^{+}}^{1-\alpha}\left(X_{0}\right)\right)+\left({ }^{C} D_{a^{+}}^{\alpha} X\right)(t),
$$

which implies

$$
\frac{\mathrm{d}}{\mathrm{d} t}\left(J_{a^{+}}^{1-\alpha}(X(t))\right)-\frac{\mathrm{d}}{\mathrm{d} t}\left(J_{a^{+}}^{1-\alpha}\left(X_{0}\right)\right)=\left({ }^{C} D_{a^{+}}^{\alpha} X\right)(t) .
$$

On the other hand, by the right-hand side of equation (7.7) and applying of [109, property (5), page 103], since $Z(t)=f(X(t), t)$ is m.s. continuous, one gets

$$
\frac{\mathrm{d}}{\mathrm{d} t}\left(J_{a^{+}}^{1}(Z(t))\right)=\frac{\mathrm{d}}{\mathrm{d} t}\left(\int_{a}^{t} Z(s) \mathrm{d} s\right)=Z(t)=f(X(t), t) .
$$

Finally, $\frac{\mathrm{d}}{\mathrm{d} t}\left(J_{a^{+}}^{1-\alpha}(X(t))\right)-\frac{\mathrm{d}}{\mathrm{d} t}\left(J_{a^{+}}^{1-\alpha}\left(X_{0}\right)\right)=\frac{\mathrm{d}}{\mathrm{d} t}\left(J_{a^{+}}^{1}(Z(t))\right)$, from (7.10) and (7.11) it follows that $\left({ }^{C} D_{a^{+}}^{\alpha} X\right)(t)=f(X(t), t)$, which means that $X(t)$ is a m.s. solution of the random fractional IVP (7.1). 


\subsection{Numerical approximations to the random fractional IVP}

This section is devoted to construct reliable discrete approximations to the solution SP of the random fractional IVP (7.1). Suppose that $X(t)$ is a second-order SP such that satisfies the random integral equation given by (7.2), hence, according to Theorem 7.1, it also satisfies IVP (7.1). Define the mesh $\left\{t_{n}: n \geq 0\right\}$ as $t_{0}:=a$ and $t_{n}:=t_{0}+n h$, being $h=(b-a) / M>0$, for a positive integer $M$ that satisfies that $t_{M}=b$. Let $n$ be any positive integer such that $0 \leq n \leq M$. Evaluating (7.2) at $t_{n}$, one gets

$$
\begin{aligned}
X\left(t_{n}\right) & =X_{0}+\frac{1}{\Gamma(\alpha)} \int_{a}^{t_{n}}\left(t_{n}-s\right)^{\alpha-1} f(X(s), s) \mathrm{d} s \\
& =X_{0}+\frac{1}{\Gamma(\alpha)} \sum_{j=0}^{n-1} \int_{t_{j}}^{t_{j+1}}\left(t_{n}-s\right)^{\alpha-1} f(X(s), s) \mathrm{d} s, \quad t_{n} \in[a, b], \quad 0<\alpha \leq 1 .
\end{aligned}
$$

It would be natural to approximate each definite integral of the sum using the forward Euler approximation. Nevertheless, this fact may lead to obtain not accurate results. If we analyse the integral of the last sum considering a fine mesh and $\alpha$ near 1 , the term $\left(t_{n}-t_{n-1}\right)^{\alpha-1}$ may not be correctly computed numerically. In this chapter, we propose an alternative by approximating only $f(s, X(x))$, $s \in\left[t_{j}, t_{j+1}\right]$ by $f\left(t_{j}, X\left(t_{j}\right)\right)$, thus

$$
\begin{aligned}
\int_{t_{j}}^{t_{j+1}}\left(t_{n}-s\right)^{\alpha-1} f(X(s), s) \mathrm{d} s & \approx \int_{t_{j}}^{t_{j+1}}\left(t_{n}-s\right)^{\alpha-1} f\left(X\left(t_{j}\right), t_{j}\right) \mathrm{d} s \\
& =\frac{h^{\alpha}}{\Gamma(\alpha+1)}\left[(n-j)^{\alpha}-(n-j+1)^{\alpha}\right] f\left(X\left(t_{j}\right), t_{j}\right) .
\end{aligned}
$$

Substituting (7.13) in (7.12), we obtain an approximation $X_{n}$ to the second-order $\mathrm{RV} X\left(t_{n}\right)$, formally representing the true solution of the random fractional IVP 
(7.1) at the time $t=t_{n} \in[a, b]$, defined as

$$
\begin{aligned}
X_{n} & =X_{0}+\frac{1}{\Gamma(\alpha)} \sum_{j=0}^{n-1} \int_{t_{j}}^{t_{j+1}}\left(t_{n}-s\right)^{\alpha-1} f\left(X_{j}, t_{j}\right) \mathrm{d} s \\
& =X_{0}+\frac{h^{\alpha}}{\alpha \Gamma(\alpha)} \sum_{j=0}^{n-1}\left[(n-j)^{\alpha}-(n-(j+1))^{\alpha}\right] f\left(X_{j}, t_{j}\right), \quad 0<\alpha \leq 1,
\end{aligned}
$$

where in the last step we have substituted the value of $\int_{t_{j}}^{t_{j+1}}\left(t_{n}-s\right)^{\alpha-1} \mathrm{~d} s$, which is well-defined since the resulting exponent is $\alpha>0$.

\subsection{Error analysis}

To study the m.s. convergence of the random numerical scheme given by equation (7.14), we introduce the sequence of errors $e_{n}$ defined as $e_{0}=0$ and $e_{n}=X_{n}-X\left(t_{n}\right)$, $n=1, \ldots, M$. We will prove that for each $t=t_{n}$ fixed, the $\lim _{h \rightarrow 0}\left\|e_{n}\right\|_{2}=0$, which means that the scheme given by equation (7.14) is m.s. convergent for every $t:=t_{n}$ in $[a, b]$. Now, consider the last expression of $X\left(t_{n}\right)$ in equation (7.12) and the first expression of $X_{n}$ in equation (7.14). By subtracting $X\left(t_{n}\right)$ from $X_{n}$, we find

$$
e_{n}=\frac{1}{\Gamma(\alpha)} \sum_{j=0}^{n-1} \int_{t_{j}}^{t_{j+1}}\left[f\left(X_{j}, t_{j}\right)-f(X(s), s)\right]\left(t_{n}-s\right)^{\alpha-1} \mathrm{~d} s .
$$

Hence, applying [109, page 102] one gets

$$
\left\|e_{n}\right\|_{2} \leq \frac{1}{\Gamma(\alpha)} \sum_{j=0}^{n-1} \int_{t_{j}}^{t_{j+1}}\left\|f\left(X_{j}, t_{j}\right)-f(X(s), s)\right\|_{2}\left(t_{n}-s\right)^{\alpha-1} \mathrm{~d} s .
$$

To accomplish our task, we will find out a bound of $\left\|f\left(X_{j}, t_{j}\right)-f(X(s), s)\right\|_{2}$ by using the hypotheses $\mathbf{N} 1$ and $\mathbf{N} 2$ on $f$. Indeed,

$$
\begin{aligned}
\left\|f\left(X_{j}, t_{j}\right)-f(X(s), s)\right\|_{2} & \leq\left\|f\left(X_{j}, t_{j}\right)-f\left(X\left(t_{j}\right), t_{j}\right)\right\|_{2} \\
& +\left\|f\left(X\left(t_{j}\right), t_{j}\right)-f\left(X(s), t_{j}\right)\right\|_{2} \\
& +\left\|f\left(X(s), t_{j}\right)-f(X(s), s)\right\|_{2}
\end{aligned}
$$




$$
\leq \kappa\left\|e_{j}\right\|_{2}+\kappa\left\|X\left(t_{j}\right)-X(s)\right\|+W(S, h) .
$$

The fundamental theorem of m.s. calculus [109, page 104] together with property (3) in [109, page 102], imply $\left\|X\left(t_{j}\right)-X(s)\right\|=\left\|\int_{s}^{t_{j}} X^{\prime}(r) d r\right\| \leq h \max _{r \in[a, b]} X^{\prime}(r)$. Notice that here we have applied that $f(X(t), t)$ is m.s. continuous, hence $X^{\prime}(t)$ is m.s. continuous, so m.s. integrable. Therefore,

$$
\left\|f\left(X_{j}, t_{j}\right)-f(X(s), s)\right\|_{2} \leq \kappa\left\|e_{j}\right\|_{2}+\gamma(h),
$$

where $\gamma(h):=\kappa h \max _{r \in[a, b]} X^{\prime}(r)+W(S, h)$. Observe that $\lim _{h \rightarrow 0} \gamma(h)=0$, since by hypothesis $\mathbf{N 2}, \lim _{h \rightarrow 0} W(S, h)=0$. Next, using the inequalities (7.15) and (7.16) we find

$$
\begin{aligned}
\left\|e_{n}\right\|_{2} & \leq \frac{1}{\Gamma(\alpha)} \sum_{j=0}^{n-1} \int_{t_{j}}^{t_{j+1}}\left(\kappa\left\|e_{j}\right\|_{2}+\gamma(h)\right)\left(t_{n}-s\right)^{\alpha-1} \mathrm{~d} s \\
& =\frac{\kappa}{\Gamma(\alpha)} \sum_{j=0}^{n-1}\left\|e_{j}\right\|_{2} \int_{t_{j}}^{t_{j+1}}\left(t_{n}-s\right)^{\alpha-1} \mathrm{~d} s \\
& +\frac{\gamma(h)}{\Gamma(\alpha)} \sum_{j=0}^{n-1} \int_{t_{j}}^{t_{j+1}}\left(t_{n}-s\right)^{\alpha-1} \mathrm{~d} s .
\end{aligned}
$$

Observe that $\int_{t_{j}}^{t_{j+1}}\left(t_{n}-s\right)^{\alpha-1} \mathrm{~d} s=\frac{h^{\alpha}}{\alpha}\left[(n-j)^{\alpha}-(n-(j+1))^{\alpha}\right]$ and $(n-j)^{\alpha}-$ $(n-(j+1))^{\alpha} \leq 1, j=0,1, \ldots, n-1$, and so $\int_{t_{j}}^{t_{j+1}}\left(t_{n}-s\right)^{\alpha-1} \mathrm{~d} s \leq \frac{h^{\alpha}}{\alpha}$. We will use the last inequality and the equality, respectively, on the right-hand side of the last expression in the inequality (7.17). Indeed,

$$
\left\|e_{n}\right\|_{2} \leq \frac{\kappa h^{\alpha}}{\alpha \Gamma(\alpha)} \sum_{j=0}^{n-1}\left\|e_{j}\right\|_{2}+\frac{\gamma(h) h^{\alpha}}{\alpha \Gamma(\alpha)} \sum_{j=0}^{n-1}\left[(n-j)^{\alpha}-(n-(j+1))^{\alpha}\right] .
$$

Taking into account the value of the following finite telescopic sum

$$
\sum_{j=0}^{n-1}\left[(n-j)^{\alpha}-(n-(j+1))^{\alpha}\right]=n^{\alpha}
$$


and $\left(t_{n}-t_{0}\right)^{\alpha}=(n h)^{\alpha}$, from (7.18) it follows that

$$
\left\|e_{n}\right\|_{2} \leq \frac{\kappa h^{\alpha}}{\alpha \Gamma(\alpha)} \sum_{j=0}^{n-1}\left\|e_{j}\right\|_{2}+\frac{\gamma(h)}{\alpha \Gamma(\alpha)}\left(t_{n}-t_{0}\right)^{\alpha} .
$$

Defining $A(h):=\frac{\kappa h^{\alpha}}{\alpha \Gamma(\alpha)}$ and $B(h):=\frac{\gamma(h)}{\alpha \Gamma(\alpha)}\left(t_{n}-t_{0}\right)^{\alpha}$, the above inequality reads

$$
\left\|e_{n}\right\|_{2} \leq A(h) \sum_{j=0}^{n-1}\left\|e_{j}\right\|_{2}+B(h), \quad n=1,2, \ldots
$$

In the following deduction, the inequality given by (7.19) will be repeatedly used. Indeed,

$$
\begin{aligned}
\left\|e_{1}\right\|_{2} & \leq A(h)\left\|e_{0}\right\|_{2}+B(h), \\
\left\|e_{2}\right\|_{2} & \leq A(h)\left\|e_{0}\right\|_{2}+B(h)+A(h)\left\|e_{1}\right\|_{2} \\
& \leq(1+A(h))\left(A(h)\left\|e_{0}\right\|_{2}+B(h)\right) .
\end{aligned}
$$

Let $k$ be an integer such that $2 \leq k<M$. Suppose that

$$
\left\|e_{j}\right\|_{2} \leq(1+A(h))^{j-1}\left(A(h)\left\|e_{0}\right\|_{2}+B(h)\right)
$$

holds for all integer $j$ such that $1 \leq j \leq k$. We will show that the inequality in (7.20) fulfils for $j=k+1$, that is

$$
\left\|e_{k+1}\right\|_{2} \leq(1+A(h))^{k}\left(A(h)\left\|e_{0}\right\|_{2}+B(h)\right) .
$$

By using the inequality given by (7.19) and the induction hypothesis given by (7.20), one gets

$$
\begin{aligned}
\left\|e_{k+1}\right\|_{2} & \leq A(h)\left\|e_{0}\right\|_{2}+B(h)+A(h) \sum_{j=1}^{k}\left\|e_{j}\right\|_{2} \\
& \leq\left(A(h)\left\|e_{0}\right\|_{2}+B(h)\right)+A(h) \sum_{j=1}^{k}(1+A(h))^{j-1}\left(A(h)\left\|e_{0}\right\|_{2}+B(h)\right)
\end{aligned}
$$




$$
=\left(A(h)\left\|e_{0}\right\|_{2}+B(h)\right)\left[1+A(h) \sum_{j=1}^{k}(1+A(h))^{j-1}\right] .
$$

Since $(1+A(h))^{k}=1+A(h) \sum_{j=1}^{k}(1+A(h))^{j-1}$,

$$
\left\|e_{k+1}\right\|_{2} \leq\left(A(h)\left\|e_{0}\right\|_{2}+B(h)\right)(1+A(h))^{k} .
$$

Then, it follows that

$$
\left\|e_{n}\right\|_{2} \leq(1+A(h))^{n-1}\left(A(h)\left\|e_{0}\right\|_{2}+B(h)\right),
$$

for all integer $n$ such that $1 \leq n \leq M$. As $\lim _{h \rightarrow 0} A(h)=\lim _{h \rightarrow 0} B(h)=0$, it follows from the inequality (7.21) that $\lim _{h \rightarrow 0}\left\|e_{n}\right\|_{2}=0$. This means that the scheme given by (7.14) is m.s. convergent at the fixed number $t=t_{n}$. In this case we say that the scheme is m.s. convergent in the fixed station sense. The next result summarizes our findings.

Theorem 7.2 With the previous notation, if the function $f$ on the right-hand side of the random fractional IVP (7.1) satisfies conditions $\mathbf{N 1}$ and $\mathbf{N 2}$, then the random fractional forward Euler-like scheme given by (7.14) is m.s. convergent to the solution of (7.1), for every $t=t_{n}:=a+n h \in[a, b]$.

\subsection{Statistical moments of the numerical approximations}

So far we have established sufficient conditions in order to guarantee the m.s. convergence of the random fractional numerical scheme (7.14). In practice, apart from constructing approximations of RFDEs it is also important to provide reliable information related to the main statistical properties of such approximations. In particular, a main goal is the computation of the mean and the variance of the approximations to the solution SP. This section is addressed to accomplish this target in the context of problem IVP (7.1). 
Taking the expectation operator, $\mathbb{E}[\cdot]$, in expression (7.14) and using its linearity, we deduce that the mean of the $X_{n}$ is given by

$$
\mathbb{E}\left[X_{n}\right]=\mathbb{E}\left[X_{0}\right]+C(\alpha) \sum_{j=0}^{n-1} a_{n j}(\alpha) \mathbb{E}\left[f\left(X_{j}, t_{j}\right)\right],
$$

where $C(\alpha)=\frac{h^{\alpha}}{\Gamma(\alpha+1)}$ and $a_{n j}(\alpha)=(n-j)^{\alpha}-(n-(j+1))^{\alpha}$.

Similarly, taking the covariance operator, $\mathbb{C o v}[\cdot, \cdot]$, and using that it is bilinear, one gets that the covariance of $X_{n}$ and $X_{m}$ is given by

$$
\begin{aligned}
\operatorname{Cov}\left[X_{n}, X_{m}\right]= & \operatorname{Cov}\left[X_{0}+C(\alpha) \sum_{j=0}^{n-1} a_{n j}(\alpha) f\left(X_{j}, t_{j}\right),\right. \\
& \left.X_{0}+C(\alpha) \sum_{i=0}^{m-1} a_{m i}(\alpha) f\left(X_{i}, t_{i}\right)\right] \\
= & \mathbb{V}\left[X_{0}\right]+C(\alpha) \sum_{i=0}^{m-1} a_{m i}(\alpha) \operatorname{Cov}\left[X_{0}, f\left(X_{i}, t_{i}\right)\right] \\
& +C(\alpha) \sum_{j=0}^{n-1} a_{n j}(\alpha) \operatorname{Cov}\left[f\left(X_{j}, t_{j}\right), X_{0}\right] \\
& +(C(\alpha))^{2} \sum_{j=0}^{n-1} \sum_{i=0}^{m-1} a_{n j}(\alpha) a_{m i}(\alpha) \operatorname{Cov}\left[f\left(X_{j}, t_{j}\right), f\left(X_{i}, t_{i}\right)\right] .
\end{aligned}
$$

Letting $m=n$ in (7.23), we obtain the variance of $X_{n}$,

$$
\begin{aligned}
\mathbb{V}\left[X_{n}\right]= & \mathbb{V}\left[X_{0}\right]+2 C(\alpha) \sum_{j=0}^{n-1} a_{n j}(\alpha) \operatorname{Cov}\left[X_{0}, f\left(X_{j}, t_{j}\right)\right] \\
& +(C(\alpha))^{2} \sum_{j=0}^{n-1} \sum_{i=0}^{n-1} a_{n j}(\alpha) a_{n i}(\alpha) \operatorname{Cov}\left[f\left(X_{j}, t_{j}\right), f\left(X_{i}, t_{i}\right)\right]
\end{aligned}
$$

where

$$
\operatorname{Cov}\left[X_{0}, f\left(X_{j}, t_{j}\right)\right]=\mathbb{E}\left[X_{0} f\left(X_{j}, t_{j}\right)\right]-\mathbb{E}\left[X_{0}\right] \mathbb{E}\left[f\left(X_{j}, t_{j}\right)\right],
$$


and

$$
\operatorname{Cov}\left[f\left(X_{j}, t_{j}\right), f\left(X_{i}, t_{i}\right)\right]=\mathbb{E}\left[f\left(X_{j}, t_{j}\right) f\left(X_{i}, t_{i}\right)\right]-\mathbb{E}\left[f\left(X_{j}, t_{j}\right)\right] \mathbb{E}\left[f\left(X_{i}, t_{i}\right)\right]
$$

\subsection{Numerical examples}

The aim of this section is to present some examples in order to illustrate the random fractional forward Euler-like method developed in the previous sections. To check its accuracy, the first example have been chosen so that the exact values for the mean and the variance can be determined, and then we can compare them against the ones provide by approximations obtained via the random fractional numerical scheme. Additionally, a similar comparative analysis is performed for the 1-PDF, which is calculated in two ways, namely, via the Random Variable Transformation technique, Theorem 4.1, which is exact, and by the approximate method exhibited in Section 6.4, which takes advantage of the PME. To analyse the accuracy of the approximations for the mean and the variance, we will use of the absolute error (AE) for the mean and for the variance,

$$
\begin{aligned}
\operatorname{AE}(\text { Mean })(t, n) & =\left|\mathbb{E}\left[X_{n}\right]-\mathbb{E}\left[X\left(t_{n}\right)\right]\right|, \\
\mathrm{AE}(\operatorname{Variance})(t, n) & =\left|\mathbb{V}\left[X_{n}\right]-\mathbb{V}\left[X\left(t_{n}\right)\right]\right|,
\end{aligned}
$$

where $\mathbb{E}\left[X\left(t_{n}\right)\right]$ and $\mathbb{V}\left[X\left(t_{n}\right)\right]$ are the exact mean and variance of the solution at time $t_{n}$, respectively, and $\mathbb{E}\left[X_{n}\right]$ and $\mathbb{V}\left[X_{n}\right]$ denote their corresponding approximations given by (7.22) and (7.24)-(7.26), respectively. As we will see later, we have chosen the absolute error as error measure since in our examples the mean values of the solutions and their variances are close to zero.

Example 7.1 Let us consider the following random fractional IVP

$$
\left\{\begin{array}{cl}
\left({ }^{C} D_{0^{+}}^{\alpha} X\right)(t) & =-X(t)+A t^{2}+X_{0}+\frac{2 A}{\Gamma(3-\alpha)} t^{2-\alpha}, \quad t \in[0,0.5], 0<\alpha \leq 1, \\
X(0) & =X_{0},
\end{array}\right.
$$

where $A$ and $X_{0}$ are independent second-order RVs. According to the IVP (7.1), $f(X, t)=-X+A t^{2}+X_{0}+\frac{2 A}{\Gamma(3-\alpha)} t^{2-\alpha}$ and is straightforward to check that $f(X, t)$ 
verifies $\mathbf{N 1}$ and $\mathbf{N 2}$. Thus, the numerical scheme (7.14) is given by

$$
\begin{gathered}
X_{n}=X_{0}+\frac{h^{\alpha}}{\Gamma(\alpha+1)} \sum_{j=0}^{n-1}\left((n-j)^{\alpha}-(n-j-1)^{\alpha}\right) \\
\cdot\left(-X_{j}+A t_{j}^{2}+X_{0}+\frac{2 A}{\Gamma(3-\alpha)} t_{j}^{2-\alpha}\right) .
\end{gathered}
$$

To calculate approximations of the mean, we apply expression (7.22), taking into account that

$$
\mathbb{E}\left[f\left(X_{j}, t_{j}\right)\right]=-\mathbb{E}\left[X_{j}\right]+t_{j}^{2} \mathbb{E}[A]+\mathbb{E}\left[X_{0}\right]+\frac{2 t_{j}^{2-\alpha}}{\Gamma(3-\alpha)} \mathbb{E}[A],
$$

while to determine approximations of the variance, we use expression (7.24)-(7.26), taking into account that

$$
\begin{aligned}
\operatorname{Cov}\left[X_{0}, f\left(X_{j}, t_{j}\right)\right]= & -\operatorname{Cov}\left[X_{0}, X_{j}\right]+\mathbb{V}\left[X_{0}\right] \\
\mathbb{C o v}\left[f\left(X_{j}, t_{j}\right), f\left(X_{i}, t_{i}\right)\right]= & \mathbb{V}\left[X_{0}\right]+V[A]\left(t_{j}^{2} t_{i}^{2}+\frac{2 t_{j}^{2} t_{i}^{2-\alpha}}{\Gamma(3-\alpha)}\right. \\
& \left.+\frac{2 t_{j}^{2-\alpha} t_{i}^{2}}{\Gamma(3-\alpha)}+4 \frac{t_{j}^{2-\alpha} t_{i}^{2-\alpha}}{\Gamma(3-\alpha)^{2}}\right) \\
& +\operatorname{Cov}\left[X_{j}, A\right]\left(-t_{i}^{2}-2 \frac{t_{i}^{2-\alpha}}{\Gamma(3-\alpha)}\right) \\
& +\operatorname{Cov}\left[X_{i}, A\right]\left(-t_{j}^{2}-2 \frac{t_{j}^{2-\alpha}}{\Gamma(3-\alpha)}\right) \\
& +\operatorname{Cov}\left[X_{j}, X_{i}\right]+\mathbb{C o v}\left[X_{j}, X_{0}\right]+\mathbb{C o v}\left[X_{i}, X_{0}\right] .
\end{aligned}
$$

At this point it is interesting to point out that, using repeatedly the recurrence (7.29) any $R V X_{i}$ and $X_{j}, 1 \leq i, j \leq n-1$, in the expression (7.31) can be expressed polynomially (observe the polynomial form of $f(X, t)$ ) in terms of the data $X_{0}$ and A. As a consequence, the variance of approximation $X_{n}$, given by (7.24)-(7.26), can be expressed via covariances whose arguments will depend polynomially of $X_{0}$ and $A$. In this manner these covariances can be approximated using direct integration for moderate values of $n$, and Monte Carlo simulations for large values of $n$. 


\begin{tabular}{|c|c|c|c|c|}
\hline & $t=0.1$ & $t=0.2$ & $t=0.3$ & $t=0.4$ \\
\hline$M=100$ & $4.77208 \mathrm{e}-03$ & $1.95806 \mathrm{e}-02$ & $4.44101 \mathrm{e}-02$ & $7.92553 \mathrm{e}-02$ \\
\hline$M=200$ & $4.88730 \mathrm{e}-03$ & $1.97919 \mathrm{e}-02$ & $4.47069 \mathrm{e}-02$ & $7.96296 \mathrm{e}-02$ \\
\hline$M=400$ & $4.94406 \mathrm{e}-03$ & $1.98965 \mathrm{e}-02$ & $4.48540 \mathrm{e}-02$ & $7.98154 \mathrm{e}-02$ \\
\hline$M=800$ & $4.97217 \mathrm{e}-03$ & $1.99484 \mathrm{e}-02$ & $4.49272 \mathrm{e}-02$ & $7.99079 \mathrm{e}-02$ \\
\hline$M=1600$ & $4.98613 \mathrm{e}-03$ & $1.99743 \mathrm{e}-02$ & $4.49637 \mathrm{e}-02$ & $7.99540 \mathrm{e}-02$ \\
\hline$M=3200$ & $4.99308 \mathrm{e}-03$ & $1.99871 \mathrm{e}-02$ & $4.49818 \mathrm{e}-02$ & $7.99770 \mathrm{e}-02$ \\
\hline
\end{tabular}

Table 7.1: Approximations of the mean calculated by expressions (7.22) and (7.30) for different values of $n$ and different time instants $t$. Example 7.1.

It is easy to check that the solution SP to the random fractional IVP (7.28) is given by

$$
X(t)=A t^{2}+X_{0}
$$

Consequently, since $A$ and $X_{0}$ are independent, the mean and the variance of $X(t)$ is given by

$$
\begin{aligned}
& \mathbb{E}[X]=t^{2} \mathbb{E}[A]+\mathbb{E}\left[X_{0}\right], \\
& \mathbb{V}[X]=t^{4} \mathbb{V}[A]+\mathbb{V}\left[X_{0}\right]
\end{aligned}
$$

To carry out computations, let us consider that $A$ has a beta distribution $A \sim$ $B e(80 ; 80)$ and $X_{0}$ has a Gaussian distribution with zero mean and standard deviation $0.1, X_{0} \sim N\left(0 ; 0.1^{2}\right)$. In this example, we will take as fractional order $\alpha=0.7 \in(0,1]$. Tables 7.1 and 7.2 collect the values of the approximations for the mean and for the variance, respectively, of the solution computed via (7.22) and (7.30) for the mean, and via (7.24) and (7.31) for the variance, at the final times instants $t=0.1,0.2,0.3,0.4$ and different nodes of discretization $M=100,200,400,800,1600,3200$. So, for $t$ fixed, the step size $h=t / M$ decreases as $M$ increases. In Tables 7.3 and 7.4 , we show the absolute errors, defined in (7.27), for the approximations of the mean and the variance, respectively. From the figures collected in these tables, we can observe that for $t$ fixed, the errors decrease as $M$ increases as expected.

So far, we have computed approximations for the main statistical moments, namely the mean and the variance, of the solution $X(t)$ via the random numerical scheme, and they have been compared with their exact values. On the one hand, using the 
Chapter 7. Mean square convergent numerical solutions of random fractional differential equations

\begin{tabular}{|c|c|c|c|c|}
\hline & $t=0.1$ & $t=0.2$ & $t=0.3$ & $t=0.4$ \\
\hline$M=100$ & $1.00323 \mathrm{e}-02$ & $1.00596 \mathrm{e}-02$ & $1.00990 \mathrm{e}-02$ & $1.01698 \mathrm{e}-02$ \\
\hline$M=200$ & $1.00197 \mathrm{e}-02$ & $1.00364 \mathrm{e}-02$ & $1.00633 \mathrm{e}-02$ & $1.01186 \mathrm{e}-02$ \\
\hline$M=400$ & $1.00074 \mathrm{e}-02$ & $1.00161 \mathrm{e}-02$ & $1.00373 \mathrm{e}-02$ & $1.00897 \mathrm{e}-02$ \\
\hline$M=800$ & $1.00670 \mathrm{e}-02$ & $1.01184 \mathrm{e}-02$ & $1.01790 \mathrm{e}-02$ & $1.02686 \mathrm{e}-02$ \\
\hline$M=1600$ & $9.96446 \mathrm{e}-03$ & $9.94269 \mathrm{e}-03$ & $9.93635 \mathrm{e}-03$ & $9.96339 \mathrm{e}-03$ \\
\hline$M=3200$ & $9.99630 \mathrm{e}-03$ & $9.99561 \mathrm{e}-03$ & $1.00056 \mathrm{e}-02$ & $1.00436 \mathrm{e}-02$ \\
\hline
\end{tabular}

Table 7.2: Approximations of the variance calculated by expressions (7.24) and (7.31) for different values of $n$ and different time instants $t$. Example 7.1.

\begin{tabular}{|c|c|c|c|c|}
\hline & $t=0.1$ & $t=0.2$ & $t=0.3$ & $t=0.4$ \\
\hline$M=100$ & $2.27923 \mathrm{e}-04$ & $4.19409 \mathrm{e}-04$ & $5.89888 \mathrm{e}-04$ & $7.44651 \mathrm{e}-04$ \\
\hline$M=200$ & $1.12701 \mathrm{e}-04$ & $2.08060 \mathrm{e}-04$ & $2.93103 \mathrm{e}-04$ & $3.70370 \mathrm{e}-04$ \\
\hline$M=400$ & $5.59350 \mathrm{e}-05$ & $1.03507 \mathrm{e}-04$ & $1.45974 \mathrm{e}-04$ & $1.84577 \mathrm{e}-04$ \\
\hline$M=800$ & $2.78325 \mathrm{e}-05$ & $5.15880 \mathrm{e}-05$ & $7.28065 \mathrm{e}-05$ & $9.20999 \mathrm{e}-05$ \\
\hline$M=1600$ & $1.38729 \mathrm{e}-05$ & $2.57419 \mathrm{e}-05$ & $3.63469 \mathrm{e}-05$ & $4.59913 \mathrm{e}-05$ \\
\hline$M=3200$ & $6.92263 \mathrm{e}-06$ & $1.28546 \mathrm{e}-05$ & $1.81559 \mathrm{e}-05$ & $2.29775 \mathrm{e}-05$ \\
\hline
\end{tabular}

Table 7.3: Absolute errors for the approximations of the mean collected in Table 7.1. Example 7.1 .

\begin{tabular}{|c|c|c|c|c|}
\hline & $t=0.1$ & $t=0.2$ & $t=0.3$ & $t=0.4$ \\
\hline$M=100$ & $3.16109 \mathrm{e}-05$ & $5.48771 \mathrm{e}-05$ & $8.13128 \mathrm{e}-05$ & $1.21075 \mathrm{e}-04$ \\
\hline$M=200$ & $1.89957 \mathrm{e}-05$ & $3.16587 \mathrm{e}-05$ & $4.56261 \mathrm{e}-05$ & $6.98054 \mathrm{e}-05$ \\
\hline$M=400$ & $6.65378 \mathrm{e}-06$ & $1.13590 \mathrm{e}-05$ & $1.96601 \mathrm{e}-05$ & $4.09081 \mathrm{e}-05$ \\
\hline$M=800$ & $6.62481 \mathrm{e}-05$ & $1.13694 \mathrm{e}-04$ & $1.61319 \mathrm{e}-04$ & $2.19851 \mathrm{e}-04$ \\
\hline$M=1600$ & $3.62636 \mathrm{e}-05$ & $6.20514 \mathrm{e}-05$ & $8.12956 \mathrm{e}-05$ & $8.53784 \mathrm{e}-05$ \\
\hline$M=3200$ & $4.41900 \mathrm{e}-06$ & $9.12639 \mathrm{e}-06$ & $1.20779 \mathrm{e}-05$ & $5.20279 \mathrm{e}-06$ \\
\hline
\end{tabular}

Table 7.4: Absolute errors for the approximations of the variance collected in Table 7.2. Example 7.1. 


\begin{tabular}{|c|c|c|c|}
\hline & $\lambda_{0}$ & $\lambda_{1}$ & $\lambda_{2}$ \\
\hline$t=0.1$ & $-2.38238 \mathrm{e}+00$ & $-4.99982 \mathrm{e}-01$ & $4.99982 \mathrm{e}+01$ \\
\hline$t=0.2$ & $-2.36349 \mathrm{e}+00$ & $-1.99934 \mathrm{e}+00$ & $4.99835 \mathrm{e}+01$ \\
\hline$t=0.3$ & $-2.28182 \mathrm{e}+00$ & $-4.49353 \mathrm{e}+00$ & $4.99281 \mathrm{e}+01$ \\
\hline$t=0.4$ & $-2.06287 \mathrm{e}+00$ & $-7.96575 \mathrm{e}+00$ & $4.97859 \mathrm{e}+01$ \\
\hline
\end{tabular}

Table 7.5: Values of parameters $\lambda_{0}, \lambda_{1}$ and $\lambda_{2}$ that, according to (6.22), determine the approximate 1-PDF using PME to the random fractional IVP (7.1) at different time instants $t=0.1,0.2,0.3,0.4$. Example 7.1 .

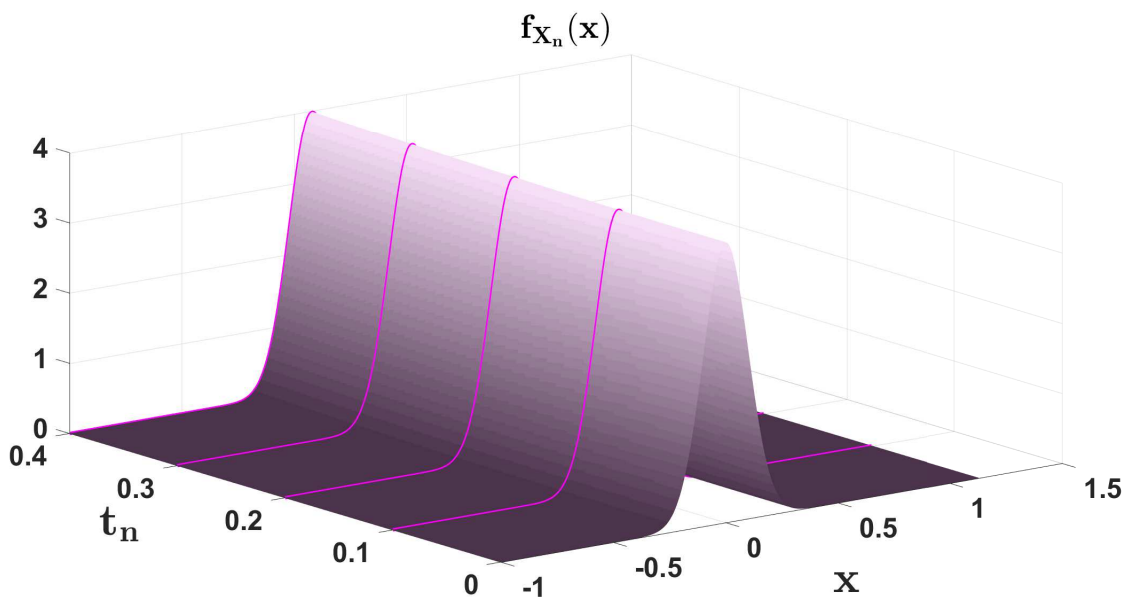

Figure 7.1: Approximate 1-PDF for the random fractional IVP (7.1) obtained via the combination of the PME and the random numerical scheme. Example 7.1.

PME described in Section 6.4 together with these approximations for the mean and for the variance, we can approximate the 1-PDF of $X(t)$ according to (6.22) where the parameters $\lambda_{i}, i=0,1,2$ solve (6.24). In Figure 7.1, we show the approximation of 1-PDF at the time interval [0,0.4]. The values of $\lambda_{0}, \lambda_{1}$ and $\lambda_{2}$, corresponding to the time instants $t=0.1,0.2,0.3,0.4$ are collected in Table 7.5.

On the other hand, as the solution SP of the random fractional IVP (7.28) is given by (7.32), its 1-PDF can be exactly calculated by applying the Random Variable Transformation technique Theorem 4.1. To this end, let us fix $t$ and define the 


\begin{tabular}{|c|c|}
\hline & Error Norm \\
\hline$t=0.1$ & $1.47402 \mathrm{e}-05$ \\
\hline$t=0.2$ & $5.89410 \mathrm{e}-05$ \\
\hline$t=0.3$ & $1.32434 \mathrm{e}-04$ \\
\hline$t=0.4$ & $2.34549 \mathrm{e}-04$ \\
\hline
\end{tabular}

Table 7.6: Values of the error (via the 2-norm) between the exact 1-PDF, given in (7.33), and its approximations obtained via PME at different times $t=0.1,0.2,0.3,0.4$. Example 7.1 .

injective mapping $r: \mathbb{R}^{2} \longrightarrow \mathbb{R}^{2}$ whose components are

$$
Y_{1}=r_{1}\left(X_{0}, A\right)=A t^{2}+X_{0}, \quad Y_{2}=r_{2}\left(X_{0}, A\right)=A
$$

Its inverse mapping, $s: \mathbb{R}^{2} \longrightarrow \mathbb{R}^{2}$, is given by

$$
X_{0}=s_{1}\left(Y_{1}, Y_{2}\right)=Y_{1}-Y_{2} t^{2}, \quad A=s_{2}\left(Y_{1}, Y_{2}\right)=Y_{2}
$$

The Jacobian of this transformation is 1 . Therefore, taking into account that $X_{0}$ and $A$ are independent $R V$ s, the joint $P D F$ of the random vector $\mathbf{Y}=\left(Y_{1}, Y_{2}\right)$ is given by

$$
f_{\mathbf{Y}}\left(y_{1}, y_{2}\right)=\left(\frac{1}{\sqrt{2 \pi 0.1^{2}}} e^{-\frac{1}{2}\left(\frac{y_{1}-y_{2} t^{2}}{0.1}\right)^{2}}\right) \frac{\left(y_{2}\right)^{79}\left(1-y_{2}\right)^{79}}{\operatorname{Be}(80,80)}
$$

where $\operatorname{Be}\left(\beta_{1}, \beta_{2}\right)$ denotes the deterministic beta special function of parameters $\beta_{1}, \beta_{2}>0$. Since the solution $X(t)$ is the first component of vector $\mathbf{Y}$, to obtain the 1-PDF of $X(t)$ we marginalize $f_{\mathbf{Y}}\left(y_{1}, y_{2}\right)$ with respect to $Y_{2}$. This yields

$$
f_{X(t)}(x)=\frac{1}{0.1 \sqrt{2 \pi} \operatorname{Be}(80,80)} \int_{0}^{1} a^{79}(1-a)^{79} e^{-\frac{1}{2}\left(\frac{x-a t^{2}}{0.1}\right)^{2}} \mathrm{~d} a .
$$

Figure 7.2 shows a graphical comparison between the exact 1-PDF, $f_{X(t)}(x)$, and its approximation at the times $t=0.1,0.2,0.3,0.4$. We can observe that approximations are very good. As a measure of the accuracy of these approximations, in Table 7.6 we show values of the 2-norm of the difference between $f_{X(t)}(x)$ and approximations $f_{X_{n}}(x)$ at the above-mentioned time instants. We observe that these figures increase at time increases as expected. 

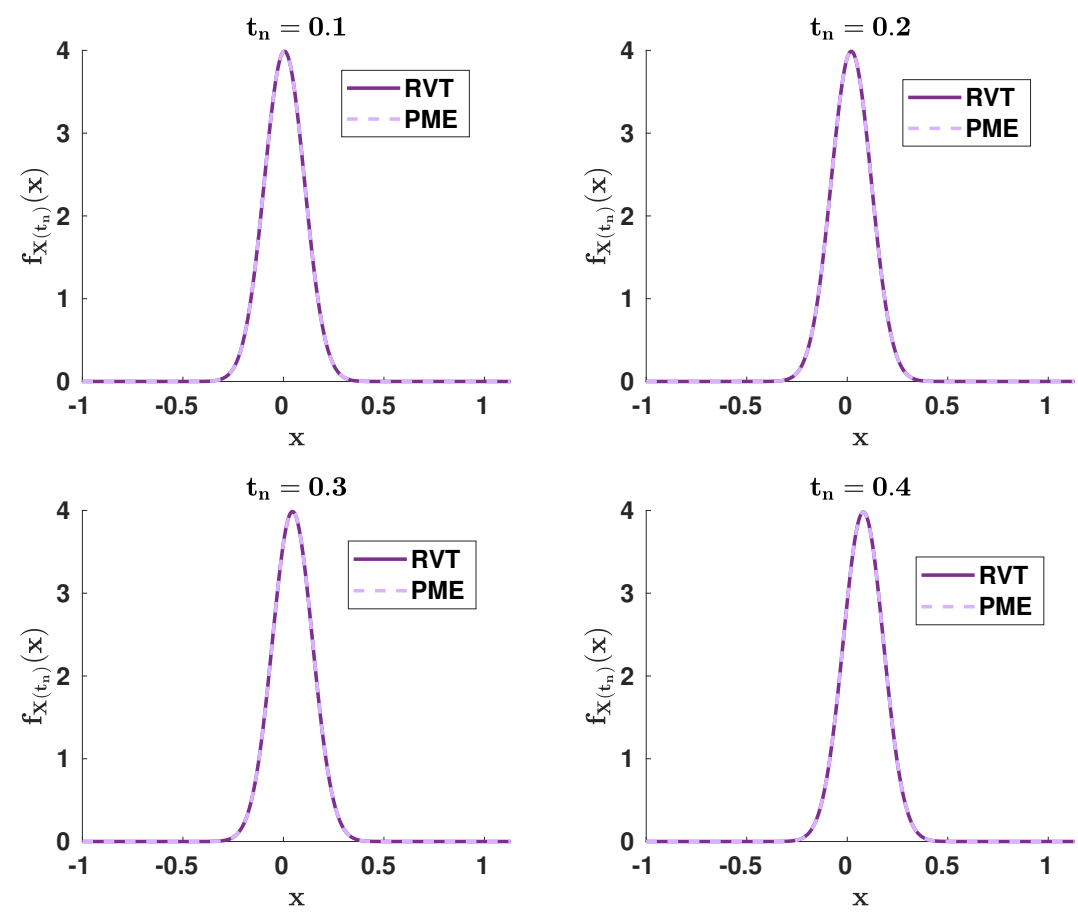

Figure 7.2: Graphical comparison between the exact 1-PDF given in (7.33) and its approximations obtained via PME at different times $t=0.1,0.2,0.3,0.4$. Example 7.1. 
Chapter 7. Mean square convergent numerical solutions of random fractional differential equations

Example 7.2 Let us consider the following random fractional IVP

$$
\left\{\begin{array}{cl}
\left({ }^{C} D_{0^{+}}^{\alpha} X\right)(t) & =\lambda X(t)+A \quad t \in[0,1], \quad 0<\alpha \leq 1, \\
X(0) & =X_{0},
\end{array}\right.
$$

where $\lambda \in \mathbb{R}$ and $X_{0}$ and $A$ are independent second-order RVs. It is easy to prove that $f(X, t)=\lambda X+A$ fulfils hypotheses $\mathbf{N 1}$ and N2. In Section 2.4 (see also [25]), the solution of this particular IVP is obtained using a generalized version of the Frobenius method. This approach leads to the following generalized power series

$$
X(t)=X_{0} \sum_{m=0}^{\infty} \frac{\lambda^{m}}{\Gamma(\alpha m+1)} t^{\alpha m}+A \sum_{m=1}^{\infty} \frac{\lambda^{m-1}}{\Gamma(\alpha m+1)} t^{\alpha m},
$$

consequently its mean and its second order moment are given by ([25, Eq. 21, 23])

$$
\begin{aligned}
\mathbb{E}[X(t)] & =\mathbb{E}\left[X_{0}\right] \sum_{m=0}^{\infty} \frac{\lambda^{m}}{\Gamma(\alpha m+1)} t^{\alpha m}+\mathbb{E}[A] \sum_{m=1}^{\infty} \frac{\lambda^{m-1}}{\Gamma(\alpha m+1)} t^{\alpha m}, \\
\mathbb{E}\left[X(t)^{2}\right] & =\mathbb{E}\left[X_{0}^{2}\right] \sum_{m=0}^{\infty} \frac{\lambda^{2 m}}{\Gamma(\alpha m+1)^{2}} t^{2 \alpha m} \\
& +2 \mathbb{E}\left[X_{0}^{2}\right] \sum_{m=0}^{\infty} \sum_{n=0}^{m-1} \frac{\lambda^{m+n}}{\Gamma(\alpha m+1) \Gamma(\alpha n+1)} t^{\alpha(m+n)} \\
& +\mathbb{E}\left[A^{2}\right] \sum_{m=1}^{\infty} \frac{\lambda^{2(m-1)}}{\Gamma(\alpha m+1)^{2}} t^{2 \alpha m} \\
& +2 \mathbb{E}\left[A^{2}\right] \sum_{m=2}^{\infty} \sum_{n=1}^{m-1} \frac{\lambda^{m+n-2}}{\Gamma(\alpha m+1) \Gamma(\alpha n+1)} t^{\alpha(m+n)} \\
& +2 \mathbb{E}\left[X_{0}\right] \mathbb{E}[A] \sum_{m=0}^{\infty} \sum_{n=1}^{m-1} \frac{\lambda^{m+n-1}}{\Gamma(\alpha m+1) \Gamma(\alpha n+1)} t^{\alpha(m+n)} .
\end{aligned}
$$

Taking into account that $\mathbb{V}[X(t)]=\mathbb{E}\left[X(t)^{2}\right]-\mathbb{E}[X(t)]^{2}$, the variance is easily obtained from the two previous expressions.

On the other hand, the numerical scheme (7.14) is given by the following expression

$$
X_{n}=X_{0}+\frac{h^{\alpha}}{\Gamma(\alpha+1)} \sum_{j=0}^{n-1}\left[(n-j)^{\alpha}-(n-(j+1))^{\alpha}\right]\left(\lambda X_{j}+A\right) .
$$




\begin{tabular}{|c|c|c|c|c|}
\hline & $t=0.2$ & $t=0.4$ & $t=0.6$ & $t=0.8$ \\
\hline$M=100$ & $8.79545 \mathrm{e}-01$ & $1.19617 \mathrm{e}+00$ & $1.53429 \mathrm{e}+00$ & $1.90780 \mathrm{e}+00$ \\
\hline$M=200$ & $8.79084 \mathrm{e}-01$ & $1.19710 \mathrm{e}+00$ & $1.53707 \mathrm{e}+00$ & $1.91297 \mathrm{e}+00$ \\
\hline$M=400$ & $8.73584 \mathrm{e}-01$ & $1.19045 \mathrm{e}+00$ & $1.52909 \mathrm{e}+00$ & $1.90346 \mathrm{e}+00$ \\
\hline$M=800$ & $8.85815 \mathrm{e}-01$ & $1.20441 \mathrm{e}+00$ & $1.54454 \mathrm{e}+00$ & $1.92030 \mathrm{e}+00$ \\
\hline$M=1600$ & $8.73489 \mathrm{e}-01$ & $1.18991 \mathrm{e}+00$ & $1.52785 \mathrm{e}+00$ & $1.90127 \mathrm{e}+00$ \\
\hline$M=3200$ & $8.76741 \mathrm{e}-01$ & $1.19452 \mathrm{e}+00$ & $1.53416 \mathrm{e}+00$ & $1.90966 \mathrm{e}+00$ \\
\hline
\end{tabular}

Table 7.7: Approximations for the mean calculated by expressions (7.22) and (7.35) for different values of $n$ at different time instants. Example 7.2.

To compute the approximations for the mean and for the variance of the numerical solution given by expressions (7.22) and (7.24)-(7.26), respectively, we need the following expressions

$$
\mathbb{E}\left[f\left(X_{j}, t_{j}\right)\right]=\lambda \mathbb{E}\left[X_{j}\right]+\mathbb{E}[A],
$$

and

$$
\begin{aligned}
\operatorname{Cov}\left[X_{0}, f\left(X_{j}, t_{j}\right)\right]= & \lambda \mathbb{C o v}\left[X_{0}, X_{j}\right], \\
\mathbb{C o v}\left[f\left(X_{j}, t_{j}\right), f\left(X_{i}, t_{i}\right)\right]= & \lambda^{2} \mathbb{C o v}\left[X_{j}, X_{i}\right]+\lambda \mathbb{C o v}\left[X_{j}, A\right] \\
& +\lambda \mathbb{C o v}\left[X_{i}, A\right]+\operatorname{Var}[A],
\end{aligned}
$$

where in the second identity we have used that $\operatorname{Cov}\left[X_{0}, A\right]=0$, since $X_{0}$ and $A$ are assumed to be independent. To carry out the numerical example, let us consider that $A \sim G a(1,1 / 2), X_{0} \sim \operatorname{Exp}(2), \alpha=0.7$ and $\lambda=0.75$. In Tables 7.7 and 7.8 , the values of the approximations for the mean and for the variance, respectively, for different $t=0.2,0.4,0.6,0.8$ and for different nodes of discretization $n=100,200,400,800,1600,3200$ are shown. In Tables 7.9 and 7.10, we collect the values of the absolute errors for the mean and for the variance, respectively. The values collected in these tables show strong agreement between both approaches, validating the approximations obtained by the numerical scheme.

Once reliable approximations for the mean and for the variance have been computed, we can take advantage for the PME explained in Section 6.4, to compute approximations of the 1-PDF of the solution for the IVP (7.34). The values of $\lambda_{0}, \lambda_{1}$ and $\lambda_{2}$ for $t=0.2,0.4,0.6,0.8$, are collected in Table 7.11. In Figure 7.3, we have plotted the approximate $1-P D F$ on the interval $0 \leq t \leq 0.8$. 
Chapter 7. Mean square convergent numerical solutions of random fractional differential equations

\begin{tabular}{|c|c|c|c|c|}
\hline & $t=0.2$ & $t=0.4$ & $t=0.6$ & $t=0.8$ \\
\hline$M=100$ & $4.73540 \mathrm{e}-01$ & $7.32315 \mathrm{e}-01$ & $1.07587 \mathrm{e}+00$ & $1.53917 \mathrm{e}+00$ \\
\hline$M=200$ & $4.83486 \mathrm{e}-01$ & $7.48728 \mathrm{e}-01$ & $1.10215 \mathrm{e}+00$ & $1.58059 \mathrm{e}+00$ \\
\hline$M=400$ & $4.58941 \mathrm{e}-01$ & $7.13128 \mathrm{e}-01$ & $1.05240 \mathrm{e}+00$ & $1.51228 \mathrm{e}+00$ \\
\hline$M=800$ & $4.76056 \mathrm{e}-01$ & $7.39889 \mathrm{e}-01$ & $1.09176 \mathrm{e}+00$ & $1.56789 \mathrm{e}+00$ \\
\hline$M=1600$ & $4.60671 \mathrm{e}-01$ & $7.19230 \mathrm{e}-01$ & $1.06591 \mathrm{e}+00$ & $1.53717 \mathrm{e}+00$ \\
\hline$M=3200$ & $4.77874 \mathrm{e}-01$ & $7.42555 \mathrm{e}-01$ & $1.09631 \mathrm{e}+00$ & $1.57625 \mathrm{e}+00$ \\
\hline
\end{tabular}

Table 7.8: Approximations for the variance calculated by expressions (7.24) and (7.36) for different values of $n$ at different time instants. Example 7.2.

\begin{tabular}{|c|c|c|c|c|}
\hline & $t=0.2$ & $t=0.4$ & $t=0.6$ & $t=0.8$ \\
\hline$M=100$ & $3.86683 \mathrm{e}-03$ & $4.05048 \mathrm{e}-03$ & $4.38730 \mathrm{e}-03$ & $4.79608 \mathrm{e}-03$ \\
\hline$M=200$ & $3.40606 \mathrm{e}-03$ & $4.97333 \mathrm{e}-03$ & $7.16724 \mathrm{e}-03$ & $9.97200 \mathrm{e}-03$ \\
\hline$M=400$ & $2.09392 \mathrm{e}-03$ & $1.67543 \mathrm{e}-03$ & $8.12368 \mathrm{e}-04$ & $4.57895 \mathrm{e}-04$ \\
\hline$M=800$ & $1.01376 \mathrm{e}-02$ & $1.22837 \mathrm{e}-02$ & $1.46425 \mathrm{e}-02$ & $1.72940 \mathrm{e}-02$ \\
\hline$M=1600$ & $2.18919 \mathrm{e}-03$ & $2.21026 \mathrm{e}-03$ & $2.05022 \mathrm{e}-03$ & $1.73005 \mathrm{e}-03$ \\
\hline$M=3200$ & $1.06352 \mathrm{e}-03$ & $2.39790 \mathrm{e}-03$ & $4.25688 \mathrm{e}-03$ & $6.65941 \mathrm{e}-03$ \\
\hline
\end{tabular}

Table 7.9: Absolute errors for the mean. Example 7.2.

\begin{tabular}{|c|c|c|c|c|}
\hline & $t=0.2$ & $t=0.4$ & $t=0.6$ & $t=0.8$ \\
\hline$M=100$ & $1.00640 \mathrm{e}-02$ & $1.36111 \mathrm{e}-02$ & $1.77728 \mathrm{e}-02$ & $2.26666 \mathrm{e}-02$ \\
\hline$M=200$ & $2.00101 \mathrm{e}-02$ & $3.00239 \mathrm{e}-02$ & $4.40562 \mathrm{e}-02$ & $6.40841 \mathrm{e}-02$ \\
\hline$M=400$ & $4.53483 \mathrm{e}-03$ & $5.57630 \mathrm{e}-03$ & $5.69099 \mathrm{e}-03$ & $4.21865 \mathrm{e}-03$ \\
\hline$M=800$ & $1.25798 \mathrm{e}-02$ & $2.11850 \mathrm{e}-02$ & $3.36613 \mathrm{e}-02$ & $5.13880 \mathrm{e}-02$ \\
\hline$M=1600$ & $2.80493 \mathrm{e}-03$ & $5.26002 \mathrm{e}-04$ & $7.81413 \mathrm{e}-03$ & $2.06665 \mathrm{e}-02$ \\
\hline$M=3200$ & $1.43977 \mathrm{e}-02$ & $2.38508 \mathrm{e}-02$ & $3.82108 \mathrm{e}-02$ & $5.97441 \mathrm{e}-02$ \\
\hline
\end{tabular}

Table 7.10: Absolute error for the variance. Example 7.2.

\begin{tabular}{|c|c|c|c|}
\hline & $\lambda_{0}$ & $\lambda_{1}$ & $\lambda_{2}$ \\
\hline$t=0.2$ & $3.61678 \mathrm{e}-01$ & $-1.88937 \mathrm{e}+00$ & $1.07880 \mathrm{e}+00$ \\
\hline$t=0.4$ & $7.42481 \mathrm{e}-01$ & $-1.65871 \mathrm{e}+00$ & $6.95697 \mathrm{e}-01$ \\
\hline$t=0.6$ & $1.05322 \mathrm{e}+00$ & $-1.44590 \mathrm{e}+00$ & $4.72548 \mathrm{e}-01$ \\
\hline$t=0.8$ & $1.32114 \mathrm{e}+00$ & $-1.25486 \mathrm{e}+00$ & $3.29706 \mathrm{e}-01$ \\
\hline
\end{tabular}

Table 7.11: Values of the parameters $\lambda_{0}, \lambda_{1}$ and $\lambda_{2}$ to construct the approximate 1-PDF using PME for the IVP (7.34) at different $t=0.2,0.4,0.6,0.8$. 


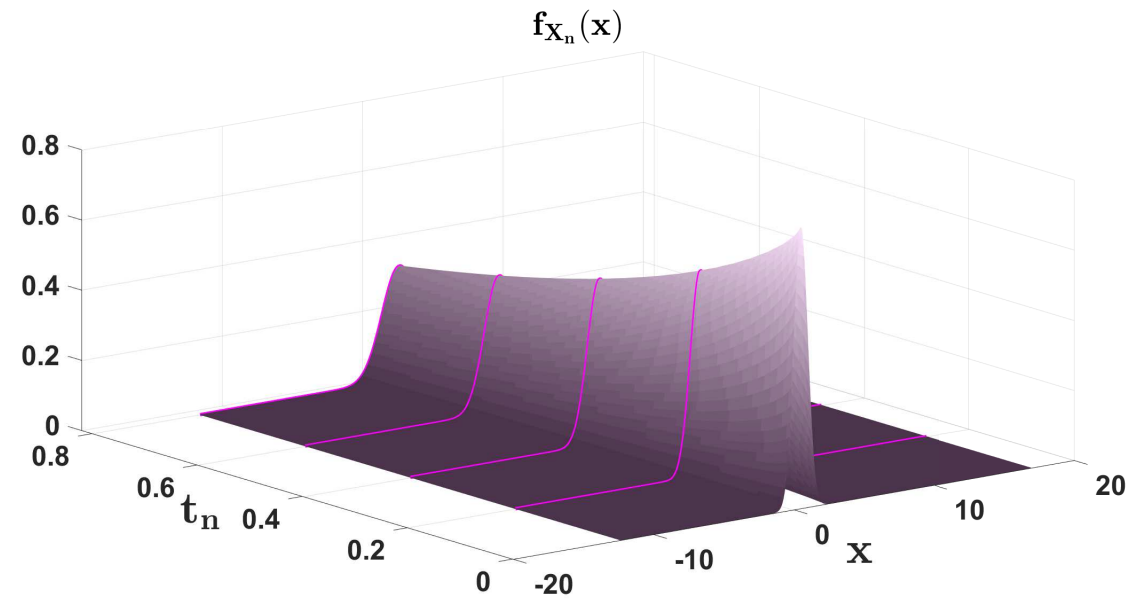

Figure 7.3: Approximate 1-PDF for the IVP (7.34) using PME combined wit the approximations of the mean and the variance obtained via the random fractional numerical scheme.

\subsection{Conclusions}

In this chapter we have studied the fractional forward Euler-like numerical method to RFDEs. The study has been conducted by means of the so-called m.s. calculus. We have given mild sufficient condition in order to guarantee the m.s. convergence. This type of stochastic convergence guarantees the mean and the variance of the approximations will converge to the corresponding exact values. This results very useful since these probabilistic moments are not known in practice. This key probabilistic information has been utilized to go further and to calculate reliable approximations of the first probability density function of the solution SP of RFDEs by applying the Principle of Maximum Entropy (PME). Our numerical examples show very satisfactory results. This chapter provides a new approach to approximate the density of RFDEs via the combination of numerical schemes and PME. We plan to extend our ideas to other types of deterministic numerical schemes for fractional differential equations like for example $[76,77,86]$ that can be formulated taking into account randomness. 

equations

\section{Chapter published}

The results of this chapter have been published in [26]. With regard to this paper, the $\mathrm{PhD}$ candidate has contributed by working in its complete development with more emphasis on the computational implementation of the fractional Euler method, constructing approximations of the 1-PDF using PME, preparing the numerical examples and participating in the writing of the paper. 


\section{Chapter}

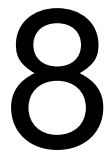

Studying the evolution over the next few years of the meningococcal genogroup $W$ in Spain using a competition Lotka Volterra model

So far, fractional calculus has been extended to the random framework from a theoretical point of view. In order to illustrate its applicability in mathematical modelling, this chapter is devoted to combine a discrete

Susceptible-Carrier-Susceptible epidemiological model with a fractional Lotka-Volterra model to describe the outbreaks of meningococcus $W$-315 infections in Spain. To do it, data retrieved from carriers of different meningococcus genogroups in Spain and their corresponding error of measurement will be taken into account. The so called Probabilistic Fitting, which is a computational technique to treat the randomness in the data, is applied to get reliable predictions. Probability distributions of the model parameters and the $95 \%$ confidence intervals of the outputs of the models are obtained to predict the evolution of the carriers of Meningococcus $W$-135 over the next few years. 
Chapter 8. Studying the evolution over the next few years of the meningococcal genogroup $W$ carriers in Spain

\subsection{Introduction}

Meningococcal disease is caused by the bacterium Neisseria meningitidis, also called meningococcus. About $10 \%$ of people have this type of bacteria in the back of their nose and throat with no signs or symptoms of the disease, being called carriers. But sometimes Neisseria meningitidis bacteria can invade the body causing certain illnesses, which are known as meningococcal diseases [70].

The disease, Meningitis, is an infection of the brain and spinal cord and can even infect the blood. Nowadays, the main cause of Meningitis is the bacterium Neisseria meningitidis. This bacterium is transmitted exclusively among humans, mainly during adolescence. An individual may get infected by contact with a carrier individual, that is, healthy carriers transmit the bacteria. It is treated with specific antibiotics, however, even properly treated, there is up to $10 \%$ of mortality and $10 \%$ of survivors have sequels $[32,48]$.

On the one hand, there are 12 types or genogroups of Meningococcus based on the capsular polysaccharides: A, B, C, H, I, K, L, X, Y, Z, E and W, but $90 \%$ of all infections are caused by types $\mathrm{A}, \mathrm{B}, \mathrm{C}, \mathrm{Y}$ and $\mathrm{W}$, being $\mathrm{A}, \mathrm{B}$ and $\mathrm{C}$ the most common (W-cases are only the $4 \%$ in US). On the other hand, the type A has been the most prevalent in Africa and Asia, but is rare in North America and Europe.

In Spain the major concern in the last decades has been the infection by the genogroups $\mathrm{C}$ and $\mathrm{B}$. In particular, Neisseria meningitidis genogroup C (MenC) was the leading cause of meningococcal disease in Spain in the late 1990s. Men C mainly impacted on the infants and toddlers populations but also on adolescents and young adults, which also acted as carriers of the disease and spreader the disease among other age groups. After controlling $\mathrm{MenC}$ by vaccination campaigns and recent revisions and booster doses, we can fairly say that MenC disease prevalence has greatly decreased in Spain [72]. On the other hand, Public Health responsibles and Meningitis experts are now concerned about the fact that the ecological niche left by the waning genogroup could be occupied by another genogroup such as B or the even more lethal, W-135. 
The genogroup W-135 origin has been traced back to Africa where it has competed with genogroup A and other in the so-called sub-Saharan Africa Meningitis belt. Since the early 2000s, the W-135 it is known to be associated with outbreaks of the Meningitis disease after the annual Hajj pilgrimage to Mecca [4]. The disease appeared also in Europe in 2000 and as early as April 2000 four children with W-135 Meningitis were treated in London. The situation became severe in Chile in 2012 when 133 cases of Invasive Meningococcal Disease were reported and the $58 \%$ of the cases the genogroup W-135 were identified. As a consequence, the fatality ratio peaked in Chile up to a $27 \%$, being the largest in the last twenty years [114]. Isolated clinical cases have also been reported in Spain since 2005, but no widespread epidemic by W-135 has occurred.

We must take into account that Neisseria meningitidis bacteria are spread through the exchange of respiratory and throat secretions like spit (e.g., living in close quarters, kissing, sharing drinks, etc). Fortunately, these bacteria are not as contagious as what causes the common cold or the flu. Besides, the bacteria are not spread by casual contact or by simply breathing the air where a person with meningococcal disease has been. Sometimes Neisseria meningitidis bacteria spreads to people who have had close or lengthy contact with a patient with meningococcal disease. People in the same household, room-mates, or anyone with direct contact with a patient's oral secretions, meaning saliva or spit, such as a boyfriend or girlfriend, would be considered at increased risk of getting the infection [70].

Meningococcus is a part of the common flora in the nasopharynx of up to $5-15 \%$ of adults and the genogroups are in competition by this ecosystem with humans. Thus, changes in health habits or prophylactic measures may change the distribution of the genogroups in this ecosystem. The study of the replacement of a genogroup by another as the most prevalent in the population is a fundamental problem in epidemiology. This is even more evident in the modern era of antibiotic resistant strains and vaccines selectively addressing a given genogroup and allowing other to proliferate.

For these reasons, in this chapter we will study a Lotka-Volterra competition model coupled to a susceptible-carrier-susceptible (SCS) model for the transmission of the meningococcal bacteria. Standard Lotka-Volterra model simulates a predator-prey 
ecological system in which, the predator and prey populations interact and regulate each other. A different version of the model with a logistic term for the evolution of the free population of each genogroup is used for the interaction of different colonies, strains or genogroups competing for the same resources (or hosts, in the case of bacteria). Instead of the original model based upon ordinary differential equations, we propose also a generalization involving fractional derivatives. Fractional calculus is a tool to describe some physical systems exhibiting hysteresis and viscoelastic properties but also other behaviour such as subdiffusion [105, 125, 124]. The order of the fractional derivative is a parameter that could help us to estimate the effect of genetic changes in the strains and recombination which enhance the adaptation to the host of the dominant genogroups.

From the epidemiological and clinical point of view, there have been some recent interest in the W-135 specially after the outbreak in Chile [114], the subsequent outbreaks associated with the Hajj's pilgrims [4] and the vaccination against W-135 carried out in UK as a prevention after the initial cases of disease in which this strain was isolated [85]. Some models for the propagation of meningococcal $\mathrm{C}$ and $\mathrm{B}$ diseases [112,39], and also for the relation among prevalence of the infection and invasive disease [64] have also been studied recently. Pérez-Breva et al. [102] have also analysed an agent-based model for the duration of immunity after vaccination of selected population age groups and the consequences of improved vaccination strategies against meningococcal $\mathrm{C}$ disease. Anyway, in the western countries, most of the cases are produced by groups B and C. Genogroups Y and W are less frequent although there are differences in their incidence in some countries. The genogroup W-135 is associated to cases and outbreaks after travelling to Mecca [4].

The increasing number of cases in countries such as Chile and UK and the high risk of mortality associated with this strain implies that the statistical modelling of this pandemic is a hot topic in epidemiology. For these reasons we will estimate the probability for an outbreak of meningococcal W-135 disease [44] in Spain using a probabilistic fitting technique for the genogroup competition SCS model discussed above. Our approach allows us to predict the maximum increase of the number of carriers expected from the seroepidemiological studies of 2011 and 2012 at the Reference Laboratory for Meningococci of the Carlos III Institute of Health 
in Spain [72]. A different, but still unsolved question, is the relationship among the number of carriers and the clinical cases of invasive meningococcal disease. According to the experts this connection is still uncertain but it is considered that even a $4 \%$ or $5 \%$ of the total population being carriers of the $\mathrm{W}-135$ genogroup could be alarming or, at least, highly worrying. The probabilistic fitting technique would allow us to show that, from the 2011 and 2012 seroepidemiological results the maximum number of carriers of W-135 in Spain would remain below 3\% in the next three years (excluding increasing immigration of infected individuals). Consequently, an outbreak of meningococcal $\mathrm{W}$ disease is unlikely in the near future.

The chapter is organized as follows: In Section 8.2 we describe the Lotka-Volterra competition model for the genogroups coupled to the SCS model for the human hosts as well as the data provided by the Spanish Reference Laboratory for Meningococci at the Carlos III Institute of Health. The probabilistic fitting technique for obtaining the statistical distribution of the model parameters in order to perform probabilistic predictions for the future is described in Section 8.3. The results and the evolution of the estimated number of carriers in the next decade is shown in Section 8.4. The chapter ends with some conclusions drawn in Section 8.5.

\subsection{Lotka-Volterra competition model and SCS epidemilogical trans- mission}

In this section we will introduce the competition model for the meningococcal genogroups as well as the susceptible-carrier-susceptible (SCS) model. These equations describe the complex ecosystem composed by all the meningococci genogroups and the human hosts. Firstly, we will give a summary of the data obtained in the seroepidemiological study for all meningococcal genogroups and, in particular, the $\mathrm{W}-135$ strain. 
Chapter 8. Studying the evolution over the next few years of the meningococcal genogroup $W$ carriers in Spain

\subsubsection{Data}

In this study, we use data provided by the Reference Laboratory for Meningococci of the Spanish Institute of Health Carlos III, collected in Tables 8.1 and 8.2, for December, 2011 and December, 2012. These are currently the only available data.

\begin{tabular}{|c|cc|}
\hline & $t_{1}=2011$ & $t_{2}=2012$ \\
\hline Sample size & 3000 & 500 \\
\hline Susceptible population & $2626(87.53 \%)$ & $409(81.8 \%)$ \\
Carrier population & $374(12.47 \%)$ & $91(18.2 \%)$ \\
\hline
\end{tabular}

Table 8.1: Sample size, number and percentage of susceptible carriers of any meningococcus in Spain in Dec 2011 and Dec 2012.

\begin{tabular}{|c|cc|}
\hline Dates & Genotype W & Other genotypes \\
\hline$t_{1}=2011$ & $4.3 \%$ & $95.7 \%$ \\
$t_{2}=2012$ & $5.5 \%$ & $94.5 \%$ \\
\hline
\end{tabular}

Table 8.2: Percentage of carriers of the genotype Men W-135 in Spain in Dec 2011 and Dec 2012 among the carriers.

\subsubsection{Susceptible-Carrier-Susceptible (SCS) model}

Firstly, we state an epidemiological model in order to describe the transmission dynamics of all the meningococci. This is a classical SIS type-model where the "infected" people is called "carrier" in this case. Carriers are people who carry meningococci bacteria and can transmit it to other people. Only when meningococci invades the host, they get infected. As we indicated, the meningococci spreads through the exchange of respiratory and throat secretions. The carriers clear the bacteria after some months becoming susceptible again. The above transmission dynamics of meningococci can be modelled by the following system of non-linear difference equations

$$
\left\{\begin{array}{l}
S_{t+1}=S_{t}-\beta S_{t} C_{t}+\gamma C_{t}, \\
C_{t+1}=C_{t}+\beta S_{t} C_{t}-\gamma C_{t},
\end{array}\right.
$$

where $S_{t}$ and $C_{t}$ are the percentage of susceptible and carriers in the month $t$, respectively, $\beta$ is the transmission rate of meningococci and $1 / \gamma$ is the average 
time in moths a carrier individual clears the bacteria. A diagram of the model can be seen in Figure 8.1.

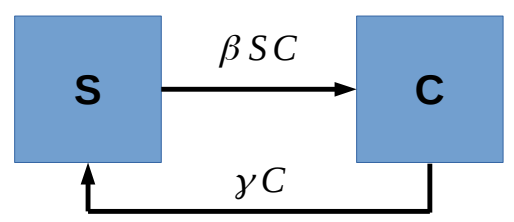

Figure 8.1: Diagram of Susceptible-Carrier-Susceptible (SCS) model in Eq. (8.1).

Taking into account that $S_{t}$ and $C_{t}$ are percentages and $S_{t}+C_{t}=1$ we can write the above system (8.1) with only the following equation

$$
C_{t+1}=(1+\beta-\gamma) C_{t}-\beta C_{t}^{2}
$$

which is a non-linear difference equation for the percentage of carriers, $C_{t}$, at month $t$.

\subsubsection{Lotka-Volterra's competition model}

The above model will give us the percentage of carriers in every time instant (in months). But meningococci are in competition in their ecosystem. Therefore, in order to understand the competition dynamics of the meningococcus $\mathrm{W}-135$ with respect to the others, we introduce the following Lotka-Volterra's continuous competition model of two species

$$
\left\{\begin{array}{l}
X_{1}^{\prime}(t)=r_{1} X_{1}(t)\left(K_{1}-X_{1}(t)\right)-\alpha_{1,2} X_{1}(t) X_{2}(t), \\
X_{2}^{\prime}(t)=r_{2} X_{2}(t)\left(K_{2}-X_{2}(t)\right)-\alpha_{2,1} X_{2}(t) X_{1}(t),
\end{array}\right.
$$

where $i=1$ corresponds to $\mathrm{W}-135$ genogroup and $i=2$ corresponds to non-W-135 genogroup, and, for $i, j=1,2$, we have:

- $X_{i}(t)$ is the total amount of the genotype $i$ meningoccocus bacteria at the time instant $t$,

- $r_{i}>0$ is the growth rate of the genotype $i$ meningoccocus bacteria, 
Chapter 8. Studying the evolution over the next few years of the meningococcal genogroup $W$ carriers in Spain

- $K_{i}>0$ is the carrying capacity of the genotype $i$ meningoccocus bacteria,

- $\alpha_{i, j}>0$ is the effect of the genotype $j$ bacteria on the growth of the genotype $i$ bacteria.

Notice that in our model we assign a label to the genogroup W-135 in which we are mostly interested, $i=1$, but the collective composed by the other genogroups is treated as a single group with $i=2$.

Taking into account that data collected in Table 8.2 are in percentages and, in order to combine the results of this model with those of the SCS model, we need scale the model (8.3). To this end, firstly, we introduce the following change of variables,

$$
x_{i}(t)=\frac{X_{i}(t)}{K_{i}}, \quad i=1,2,
$$

which represents the percentage of ecosystem occupied by the $i$-th-genogroup. It is easy to check that $0 \leq x_{i}(t) \leq 1$ and $x_{1}(t)+x_{2}(t)=1$.

Introducing the change of variable in Eq. (8.4) into Eq. (8.3), this latter system can be written as

$$
\left\{\begin{array}{l}
x_{1}^{\prime}(t)=r_{1} x_{1}(t) K_{1}\left(1-x_{1}(t)\right)-\alpha_{1,2} K_{2} x_{1}(t) x_{2}(t), \\
x_{2}^{\prime}(t)=r_{2} x_{2}(t) K_{2}\left(1-x_{2}(t)\right)-\alpha_{2,1} K_{1} x_{1}(t) x_{2}(t) .
\end{array}\right.
$$

Finally, if we define $H_{i}=r_{i} K_{i}>0, i=1,2, M_{1}=\alpha_{1,2} K_{2}>0$ and $M_{2}=\alpha_{2,1} K_{1}>$ 0 , we have

$$
\left\{\begin{array}{l}
x_{1}^{\prime}(t)=H_{1} x_{1}(t)\left(1-x_{1}(t)\right)-M_{1} x_{1}(t) x_{2}(t), \\
x_{2}^{\prime}(t)=H_{2} x_{2}(t)\left(1-x_{2}(t)\right)-M_{2} x_{1}(t) x_{2}(t),
\end{array}\right.
$$

where $H_{i}$ and $M_{i}, i=1,2$, are the new parameters of the system. As in the SCS model, taking into account that $x_{1}(t)+x_{2}(t)=1$, we only consider the first equation of (8.6), related with the dynamics of the meningococcus W-135,

$$
x_{1}^{\prime}(t)=H_{1} x_{1}(t)\left(1-x_{1}(t)\right)-M_{1} x_{1}(t)\left(1-x_{1}(t)\right),
$$


that can be simplified as

$$
x_{1}^{\prime}(t)=N_{1} x_{1}(t)\left(1-x_{1}(t)\right),
$$

where $N_{1}=H_{1}-M_{1}$. So, the competition dynamics is basically governed by the Ricatti equation (8.7) in which the parameter $N_{1}$ is given by the difference among the reproductive factor for the $\mathrm{W}-135$ genogroup, $H_{1}$, and the competition parameter with the other genogroups, $M_{1}$.

\section{The fractional Lotka-Volterra model}

As we have mentioned in the introduction, the competition dynamics may reinforce certain genogroups by DNA recombination or mutations and this would depend on the other genogroups coexisting with them as well as the time this coexistence lasts and their populations. In this spirit, we will extend the original Lotka-Volterra model to a generalized one in which ordinary derivatives are replaced by fractional derivatives. It is well-known that fractional differential equations may account for these memory effects as they are usually applied to visco-elastic materials and sub-diffusive processes [105, 125].

So, we replace the first derivative of model (8.7) by a fractional Caputo derivative of order $\alpha(0<\alpha<1)[10]$. This way, the model will be given by the following expression:

$$
{ }^{C} D^{\alpha} x_{1}(t)=N_{1} x_{1}(t)\left(1-x_{1}(t)\right),
$$

where ${ }^{C} D_{0}^{\alpha} f$ denotes the fractional Caputo derivative of order $\alpha$ of the function $f:[0, \infty[\rightarrow \mathbb{R}$, described (2.16). Discretizing Eq. (8.8), we obtain the following expression,

$$
\left(\Delta_{*}^{\alpha} x_{1}\right)(t)=N_{1} x_{1}(t+\alpha-1)\left(1-x_{1}(t+\alpha-1)\right)
$$

where $t \in \mathbb{N}_{1-\alpha}:=\{1-\alpha, 2-\alpha, \ldots\}$, and $\Delta_{\alpha}^{*}$ is the Caputo-like delta difference operator which, according to Atici and Eloe [10], represents the Caputo derivative 
Chapter 8. Studying the evolution over the next few years of the meningococcal genogroup $W$ carriers in Spain

in discrete time. This difference is defined by the following expression

$$
\left(\Delta_{*}^{\alpha} x_{1}\right)(t)=\frac{1}{\Gamma(1-\alpha)} \sum_{s=0}^{t-n+\alpha} \frac{\Gamma(t-s)}{\Gamma(t-s+1)}\left(\Delta x_{1}\right)(s),
$$

where $\left(\Delta x_{1}\right)(t)$ is the discretization of the first derivative of $x_{1}(s)$ in discrete time, i.e.,

$$
\left(\Delta x_{1}\right)(s)=x_{1}(s)-x_{1}(s-1) .
$$

Moreover, by [57, Lemma 2.4], the difference system (8.9) can be rewritten as

$$
\begin{aligned}
x_{1}(t) & =x_{1}(0)+\frac{1}{\Gamma(\alpha)} \sum_{s=1-\alpha}^{t-\alpha} \frac{\Gamma(t-s)}{\Gamma(t-s-\alpha+1)} N_{1} x_{1}(s+\alpha-1)\left(1-x_{1}(s+\alpha-1)\right) \\
& =x_{1}(0)+\frac{1}{\Gamma(\alpha)} \sum_{s=1}^{t} \frac{\Gamma(t-k+\alpha)}{\Gamma(t-k+1)} N_{1} x_{1}(k-1)\left(1-x_{1}(k-1)\right),
\end{aligned}
$$

where $t$ is a positive integer. Denoting $x_{1}(t)=x_{t}$, the fractional Lotka-Volterra model in discrete time is formulated as

$$
x_{t}=x_{0}+\frac{1}{\Gamma(\alpha)} \sum_{s=1}^{t} \frac{\Gamma(t-k+\alpha)}{\Gamma(t-k+1)} N_{1} x_{k-1}\left(1-x_{k-1}\right),
$$

where $N_{1}$ and $\alpha$ are the model parameters to be determined and $t$ is the time in months. Notice that our competition-epidemiological model involves now four parameters: the infection rate, $\beta$; the recovery rate, $\gamma$; the balance among the $\mathrm{W}-135$ genogroup reproduction and its competition parameter, $N_{1}$; and also the fractional index, $\alpha$, for the Caputo derivative in Eq. (8.13). In the next section we will show how to obtain probabilistic estimations of these four parameters from the data collected in Tables 8.1 and 8.2. From these fitting we will be able to predict reliable bounds on the evolution of $\mathrm{W}-135$ prevalence with $95 \%$ confidence and, consequently, to quantify the risk of outbreaks in the near future. 


\subsection{Probabilistic Fitting}

This technique, introduced in [44], consists of using information from surveys to assign probability distributions to the data. Then, we sample data values from these probability distributions and we fit the model to these sampled data. Thus, we find model parameters that fit not only the data but also the uncertainty contained into the intrinsic survey error. Hence, these fitted model parameters will allow the model to capture the data uncertainty (with $95 \%$ confidence intervals).

To conduct our study, probabilistic fitting technique is applied in models (8.2) and (8.13). To assingn reliable probabilistic distributions to the input data,. the following remarks will be required.

Remark 8.1 (A key property of the binomial distribution) Let $X$ be $a$ binomial $R V$ of parameters $n$ and $p, X \sim B i(n ; p)$. Then, it results as the sum of $n$ independent and identically distributed (iid) Bernoulli RVs of parameter $p$, $X_{i} \sim \operatorname{Ber}(p)$, i.e.

$$
X=\sum_{i=1}^{n} X_{i}, \quad X_{i}=\left\{\begin{array}{llc}
0 & \text { with probability } & 1-p, \\
1 & \text { with probability } & p,
\end{array} \quad 1 \leq i \leq n .\right.
$$

Observe that $Y_{i}=1-X_{i} \sim \operatorname{Ber}(1-p), 1 \leq i \leq n$. Therefore,

$$
Y=\sum_{i=1}^{n} Y_{i}=n-\sum_{i=1}^{n} X_{i}=n-X \sim B i(n ; 1-p)
$$

Remark 8.2 (A key property of the beta distribution) Let $X$ be a beta distribution of parameters $\beta_{1}>0$ and $\beta_{2}>0, X \sim B e\left(\beta_{1} ; \beta_{2}\right)$. Then, its PDF is given by

$$
f_{X}(x)=\frac{\Gamma\left(\beta_{1}+\beta_{2}\right)}{\Gamma\left(\beta_{1}+\beta_{2}\right)} x^{\beta_{1}-1}(1-x)^{\beta_{2}-1}, \quad 0<x<1 .
$$

Let us consider the $R V Y=1-X$. Applying the $R V$ transformation technique [99, Chapter 5], we can derive the PDF of $Y, f_{Y}(y)$. Observe that the mapping $r:[0,1] \rightarrow[0,1]$ defined by $y=r(x)=1-x$ is bijective, its inverse is $s(y)=1-y$ and its Jacobian is non-zero, $\frac{\mathrm{d} s(y)}{\mathrm{d} y}=-1 \neq 0$. Then, according to the so-called 
Chapter 8. Studying the evolution over the next few years of the meningococcal genogroup $W$ carriers in Spain

Fundamental Theorem [99, page 93] one gets,

$$
f_{Y}(y)=f_{X}(s(y))\left|\frac{\mathrm{d} s(y)}{\mathrm{d} y}\right|=\frac{\Gamma\left(\beta_{1}+\beta_{2}\right)}{\Gamma\left(\beta_{1}\right) \Gamma\left(\beta_{2}\right)}(1-y)^{\beta_{1}-1} y^{\beta_{2}-1}, \quad 0<y<1 .
$$

As a consequence, we have proved that if $X \sim \operatorname{Be}\left(\beta_{1} ; \beta_{2}\right)$, then $1-X \sim\left(\beta_{2} ; \beta_{1}\right)$.

\subsubsection{Data $95 \%$ confidence intervals (95\% Cl)}

Data in Table 8.1 correspond to the mean percentage of carriers in Dec 2011 and Dec 2012. Also, the sample sizes are 3000 and 500, respectively.

Assuming that the surveys are independent, for each one of the two available surveys, let us denote by $\mathbf{X}^{j}=\left(X_{1}^{j}, X_{2}^{j}\right), 0 \leq X_{i}^{j} \leq n_{j}, i, j=1,2$, a random vector whose entries are $X_{1}^{j}=$ \# Carriers, $X_{2}^{j}=$ \# Susceptible, and $n_{1}=3000$ and $n_{2}=500$ being the sample sizes of the surveys corresponding to December $2011(j=1)$ and December $2012(\mathrm{j}=2)$, respectively.

For the sake of clarity, let us fix $j=1$ and let us consider the random vector $\mathbf{X}^{1}$, but the same findings to be presented in the following, apply to the random vector $\mathbf{X}^{2}$ $(j=2)$. Initially, it is natural to assume that the $\mathrm{RV} X_{1}^{1}$ has a binomial distribution of parameters $n_{1}=3000$ and $p_{1}, X_{1}^{1} \sim \operatorname{Bi}\left(3000 ; p_{1}\right)$. As a consequence, by Remark 8.1. $X_{2}^{1}=n-X_{1}^{1} \sim \operatorname{Bi}\left(3000,1-p_{1}\right)$. However, as the available information from the data surveys is limited to December $2011(j=1)$ and December 2012 $(j=2)$ only, we are going to consider that the parameter $p_{1}$ is a RV rather than a deterministic value. Furthermore, since $p_{1} \in(0,1)$ we will assume that $p_{1}$ has a beta distribution of parameters $\beta_{1}^{1}$ and $\beta_{2}^{1}, p_{1} \sim \operatorname{Be}\left(\beta_{1}^{1} ; \beta_{2}^{1}\right)$. At this time, it is worth pointing out that beta distribution is a two parametric probabilistic distribution whose domain is just the interval $(0,1)$, then allowing for enough flexibility to describe, from a probabilistic standpoint, the parameter $p_{1}$. This approach leads us to assume that $X_{1}^{1}$ has a beta-binomial distribution

$$
X_{1}^{1} \sim \operatorname{Bi}\left(3000 ; p_{1}\right), \quad p_{1} \sim \operatorname{Be}\left(\beta_{1}^{1} ; \beta_{2}^{1}\right),
$$


whose probability mass function is given by

$$
\mathbb{P}\left[X_{1}^{1}=k\right]=\left(\begin{array}{c}
3000 \\
k
\end{array}\right) \frac{B\left(k+\beta_{1}^{1} ; 3000-k+\beta_{2}^{1}\right)}{B\left(\beta_{1}^{1} ; \beta_{2}^{1}\right)}, k=0,1, \ldots, 3000,
$$

and $B(a ; b)$ is the standard beta function defined by $B(a ; b):=\int_{0}^{1} t^{a-1}(1-t)^{b-1} \mathrm{~d} t$. As a consequence of the results collected in Remark 8.2 for the second component of the random vector $\mathbf{X}_{\mathbf{1}}$ one gets

$$
X_{2}^{1}=3000-X_{1}^{1} \sim \operatorname{Bi}\left(3000,1-p_{1}\right), 1-p_{1} \sim \operatorname{Be}\left(\beta_{2}^{1} ; \beta_{1}^{1}\right),
$$

or equivalently, with $p_{1} \sim \operatorname{Be}\left(\beta_{1}^{1} ; \beta_{2}^{1}\right)$. Hence, the probability mass function of $X_{2}^{1}$ is given by

$$
\mathbb{P}\left[X_{2}^{1}=k\right]=\left(\begin{array}{c}
3000 \\
k
\end{array}\right) \frac{B\left(k+\beta_{2}^{1} ; 3000-k+\beta_{1}^{1}\right)}{B\left(\beta_{1}^{1} ; \beta_{2}^{1}\right)}, k=0,1, \ldots, 3000,
$$

where the symmetry property of the beta function $B\left(\beta_{2}^{1} ; \beta_{1}^{1}\right)=B\left(\beta_{1}^{1} ; \beta_{2}^{1}\right)$ has been used to express the denominator of (8.15).

An analogous development follows for the random vector $\mathbf{X}^{2}=\left(X_{1}^{2}, X_{2}^{2}\right)$,

$$
\begin{array}{r}
X_{1}^{2} \sim \operatorname{Bi}\left(500 ; p_{2}\right), X_{2}^{2} \sim \operatorname{Bi}\left(500 ; 1-p_{2}\right), \quad p_{2} \sim \operatorname{Be}\left(\beta_{1}^{2} ; \beta_{2}^{2}\right), \\
\mathbb{P}\left[X_{1}^{2}=k\right]=\left(\begin{array}{c}
500 \\
k
\end{array}\right) \frac{B\left(k+\beta_{1}^{2} ; 500-k+\beta_{2}^{2}\right)}{B\left(\beta_{1}^{2} ; \beta_{2}^{2}\right)}, k=0,1, \ldots, 500, \\
\mathbb{P}\left[X_{2}^{2}=k\right]=\left(\begin{array}{c}
500 \\
k
\end{array}\right) \frac{B\left(k+\beta_{2}^{2} ; 500-k+\beta_{1}^{2}\right)}{B\left(\beta_{1}^{2} ; \beta_{2}^{2}\right)}, k=0,1, \ldots, 500 .
\end{array}
$$

In order to estimate the parameters $\left\{\beta_{1}^{1}, \beta_{2}^{1}\right\}$ of the $\operatorname{RV} p_{1} \sim \operatorname{Be}\left(\beta_{1}^{1} ; \beta_{2}^{1}\right)$ (and hence of $X_{1}^{1}$ and $X_{2}^{1}$ ), firstly we observe, from Table 8.1 that in December of 2011 $(j=1)$, the number of carriers was 374 out of 3000 individuals. Hence a reasonable punctual estimation of $p_{1}$ is $\frac{374}{3000}$. To provide estimates $\left(\hat{\beta}_{1}^{1}, \hat{\beta}_{2}^{1}\right)$ of $\left(\beta_{1}^{1}, \beta_{2}^{1}\right)$, we will consider the synthetic sample $\left\{\frac{372}{3000}, \frac{373}{3000}, \frac{374}{3000}, \frac{375}{3000}, \frac{376}{3000}\right\}$ centred at $\frac{374}{3000}$ and 
Chapter 8. Studying the evolution over the next few years of the meningococcal genogroup $W$ carriers in Spain

then we apply the maximum likehood estimation (MLE) technique. This turns out

$$
\hat{\beta}_{1}^{1}=6.122 \cdot 10^{4} \hat{\beta}_{2}^{1}=4.2984 \cdot 10^{5} .
$$

These estimates satisfy the following desirable property

$$
\mathbb{E}\left[p_{1}\right]=\frac{\beta_{1}^{1}}{\beta_{1}^{1}+\beta_{2}^{1}}=\frac{6.122 \cdot 10^{4}}{6.122 \cdot 10^{4}+4.2984 \cdot 10^{5}}=\frac{374}{3000} .
$$

Following the analogous strategy, we have constructed the estimates $\left(\hat{\beta}_{1}^{2}, \hat{\beta}_{2}^{2}\right)$ for the parameters $\left(\beta_{1}^{2}, \beta_{2}^{2}\right)$ of the $\operatorname{RV} p_{2} \sim \operatorname{Be}\left(\beta_{1}^{2} ; \beta_{2}^{2}\right)$ and hence of $\left(X_{1}^{2}\right.$ and $\left.X_{2}^{2}\right)$. Now, we have considered the sample $\left\{\frac{89}{500}, \frac{90}{500}, \frac{91}{500}, \frac{92}{500}, \frac{93}{500}\right\}$ centred at $\frac{91}{500}$. This figure comes from Table 1, since in December $2012(\mathrm{j}=2)$, the number of sample data being carrier was 91 out of 500 individuals.In this case, the application of MLE method turns out the estimates

$$
\hat{\beta}_{1}^{2}=3.387 \cdot 10^{3} \quad \hat{\beta}_{2}^{2}=1.5221 \cdot 10^{4} .
$$

This estimates also satisfies $\mathbb{E}\left[p_{2}\right]=\hat{\beta}_{1}^{2} /\left(\hat{\beta}_{1}^{2}+\hat{\beta}_{2}^{2}\right)=\frac{91}{500}$.

Now we compute the percentiles 2.5 and 97.5 in order to determine $95 \%$ CI (confidence intervals) of each random vector $\mathbf{X}^{1}=\left(X_{1}^{1}, X_{2}^{1}\right)$ and $\mathbf{X}^{2}=\left(X_{1}^{2}, X_{2}^{2}\right)$. These intervals are computed using the probability mass functions given by (8.14)(8.17) and the estimates (8.18)-(8.19). The 95\% CI are collected in Table 8.3.

\begin{tabular}{|c|cc|}
\hline Dates & Carriers $\left(X_{1}^{j}\right)$ & Susceptible $\left(X_{2}^{j}\right)$ \\
\hline$t_{1}=2011(j=1)$ & {$[11.30 \%, 13.67 \%]$} & {$[86.33 \%, 88.73 \%]$} \\
$t_{2}=2012(j=2)$ & {$[14.8 \%, 21.60 \%]$} & {$[78.20 \%, 85.20 \%]$} \\
\hline
\end{tabular}

Table 8.3: $95 \%$ CI of the data surveys using the probability mass function of Beta-Binomial distributions given by (8.14)-(8.17).

We apply the same method explained above but considering $Y_{1}^{j}=\#$ Carriers of Meningococcus $\mathrm{W}, Y_{2}^{j}=\#$ Carriers of non-Meningococcus $\mathrm{W}$ and $n_{1}=3000$ and $n_{2}=500$. Therefore, we assume that

$$
Y_{1}^{j} \sim \operatorname{Bi}\left(n_{j} ; p_{j}\right), \quad p_{j} \sim \operatorname{Be}\left(\gamma_{1}^{j} ; \gamma_{2}^{j}\right), \quad j=1,2,
$$


and consequently,

$$
Y_{2}^{j} \sim \operatorname{Bi}\left(n_{j} ; 1-p_{j}\right), \quad 1-p j \sim \operatorname{Be}\left(\gamma_{2}^{j} ; \gamma_{1}^{j}\right) \quad j=1,2
$$

Thus, their probability mass functions are given by

$$
\begin{aligned}
& \mathbb{P}\left[Y_{1}^{j}=k\right]=\left(\begin{array}{c}
n_{j} \\
k
\end{array}\right) \frac{B\left(k+\gamma_{1}^{j} ; n_{j}-k+\gamma_{2}^{j}\right)}{B\left(\gamma_{1}^{j} ; \gamma_{2}^{j}\right)}, k=0,1, \ldots, n_{j}, \quad j=1,2, \\
& \mathbb{P}\left[Y_{2}^{j}=k\right]=\left(\begin{array}{c}
n_{j} \\
k
\end{array}\right) \frac{B\left(k+\gamma_{2}^{j} ; n_{j}-k+\gamma_{1}^{j}\right)}{B\left(\gamma_{1}^{j} ; \gamma_{2}^{j}\right)}, k=0,1, \ldots, n_{j}, \quad j=1,2 .
\end{aligned}
$$

The parameters $\gamma_{i}^{j}, 1 \leq i, j \leq 2$, are estimated using the MLE technique and we have obtained

$$
\gamma_{1}^{1}=121.9, \quad \gamma_{2}^{1}=2727.7 ; \quad \gamma_{1}^{2}=11.1872, \quad \gamma_{2}^{2}=192.4464
$$

In Table 8.4, we show the 95\% CI for each the RVs $\left\{Y_{1}^{1}, Y_{1}^{2}, Y_{2}^{1}, Y_{2}^{2}\right\}$ computing the percentiles 2.5 and 97.5 of each random vector $\mathbf{Y}^{1}=\left(Y_{1}^{1}, Y_{2}^{1}\right)$ and $\mathbf{Y}^{2}=\left(Y_{1}^{2}, Y_{2}^{2}\right)$.

\begin{tabular}{|c|cc|}
\hline Dates & Genotype $\mathrm{W}\left(Y_{1}^{j}\right)$ & Genotype non-W $\left(Y_{2}^{j}\right)$ \\
\hline$t_{1}=2011(j=1)$ & {$[3.52 \%, 4.97 \%]$} & {$[95.03 \%, 96.43 \%]$} \\
$t_{2}=2012(j=2)$ & {$[3.8 \%, 7.7 \%]$} & {$[92.30 \%, 96.2 \%]$} \\
\hline
\end{tabular}

Table 8.4: $95 \%$ CI of the data surveys using the probability mass function of Beta-Binomial distributions given by $(8.20)-(8.21)$.

\subsubsection{Probabilistic estimation}

Let $\mathbb{M}_{1}(t ; \beta, \gamma)$ and $\mathbb{M}_{2}\left(t ; \alpha, N_{1}\right)$ denote short representations of model (8.2) and (8.13), respectively, where $\{\beta, \gamma\}$, and $\left\{\alpha, N_{1}\right\}$ are the models parameters of $\mathbb{M}_{1}$ and $\mathbb{M}_{2}$, respectively, and $t$ is the time in months.

Also, we have the data $(95 \%$ CI) collected in Tables 8.3 and 8.4 obtained by sampling the probability mass functions given by (8.14)-(8.17) and (8.20)-(8.21), respectively, and calculating the percentiles 2.5 and 97.5 . 
For the probability distributions given in Eq. (8.14)-(8.17), we take a sample $d_{t_{i}}^{*}$, $i, j=1,2$ and we search for the values of the model parameters $\beta^{*}, \gamma^{*}$ such that

$$
\left\|\left[\begin{array}{c}
\mathbb{M}_{1}\left(t_{1} ; \beta^{*}, \gamma^{*}\right) \\
\mathbb{M}_{1}\left(t_{2} ; \beta^{*}, \gamma^{*}\right)
\end{array}\right]-\left[\begin{array}{c}
d_{t_{1}}^{*}, d_{t_{1} 2}^{*} \\
d_{t_{2}}^{*}, d_{t_{2} 2}^{*}
\end{array}\right]\right\|_{2}
$$

is as minimum as possible, being $\|\cdot\|_{2}$ the $2-$ norm [116].

This procedure is a classic optimization problem that has been carried out through the Particle Swarm Optimization (PSO) algorithm [80], with the difference that we will not fit the data itself, but samples taken from the data's probability distributions. We shall perform the fitting $N$ times ( $N$ being a large number), storing both the parameter values $\beta^{*}, \gamma^{*}$ as well as the calculated errors $e^{*}$, ordered from smallest to largest values. The result of this procedure is a list of model parameters fitted to a sample of the data with their corresponding errors, represented in Table 8.5.

\begin{tabular}{c|c|c} 
Error & Parameters & Model \\
\hline$e_{1}^{*}$ & $\beta^{1}, \gamma^{1}$ & $\mathbb{M}_{1}\left(t ; \beta^{1}, \gamma^{1}\right)$ \\
$e_{2}^{*}$ & $\beta^{2}, \gamma^{2}$ & $\mathbb{M}_{1}\left(t ; \beta^{2}, \gamma^{2}\right)$ \\
$\vdots$ & $\vdots$ & $\vdots$ \\
$e_{N}^{*}$ & $\beta^{N}, \gamma^{N}$ & $\mathbb{M}_{1}\left(t ; \beta^{N}, \gamma^{N}\right)$ \\
\hline
\end{tabular}

Table 8.5: Model fitting to $N$ samples of the data's probability distributions.

The value $N$ should be a large number in order to capture as more data uncertainty as possible during the sampling process and this uncertainty could be fitted by the model. In this case, $N=10000$.

Now, we take $\mathbb{M}_{1}\left(t ; \beta^{1}, \gamma^{1}\right)$ and $\mathbb{M}_{1}\left(t ; \beta^{2}, \gamma^{2}\right)$ in Table 8.5 and calculate the outputs for times $t_{1}=0$ (Dec 2011) and $t_{2}=11$ (Dec 2012), the time instants (months) where data are available. For each time instant, we shall calculate percentiles 2.5 and 97.5 for carriers. Hence, we will name $m_{2}$ the sum of:

- the 2 -norm of the difference between the percentiles 2.5 from the model output and from the data percentiles 2.5 in Table 8.3 , and 
- the 2-norm of the difference between the percentiles 97.5 from the model output and from the data percentiles 97.5 in Table 8.3.

We will repeat the above process with the outputs from $\mathbb{M}_{1}\left(t ; \beta^{1}, \gamma^{1}\right), \mathbb{M}_{1}\left(t ; \beta^{2}, \gamma^{2}\right)$ and $\mathbb{M}_{1}\left(t ; \beta^{3}, \gamma^{3}\right)$, obtaining $m_{3}$, the measure between the confidence intervals from the outputs and the data. The same for $m_{4}$ and so on, until $\mathbb{M}_{1}\left(t ; \beta^{1}, \gamma^{1}\right)$, $\mathbb{M}_{1}\left(t ; \beta^{2}, \gamma^{2}\right), \ldots, \mathbb{M}_{1}\left(t ; \beta^{N}, \gamma^{N}\right)$, obtaining $m_{N}$ as the measure between the confidence intervals from the outputs and the data.

Taking $m_{k}=\min \left\{m_{1}, \ldots, m_{N}\right\}$, we ensure that the $95 \%$ CI bands of the outputs from $\mathbb{M}_{1}\left(t ; \beta^{1}, \gamma^{1}\right), \mathbb{M}_{1}\left(t ; \beta^{2}, \gamma^{2}\right), \ldots, \mathbb{M}_{1}\left(t ; \beta^{k}, \gamma^{k}\right)$ is the closest to the $95 \%$ confidence intervals from the data, with which our model will capture the maximum possible uncertainty of the data from the output of the models $\mathbb{M}_{1}\left(t ; \beta^{1}, \gamma^{1}\right)$, $\mathbb{M}_{1}\left(t ; \beta^{2}, \gamma^{2}\right), \ldots, \mathbb{M}_{1}\left(t ; \beta^{k}, \gamma^{k}\right)$.

This procedure should be repeated for model $\mathbb{M}_{2}\left(t ; \alpha, N_{1}\right)$ by sampling the probability mass functions given by Eq. (8.20)-(8.21).

\subsection{Results}

For the SCS model $\mathbb{M}_{1}(t ; \beta, \gamma)$, we obtain $m_{k}=5211$. With the outputs of $\mathbb{M}_{1}\left(t ; \beta^{1}, \gamma^{1}\right), \ldots, \mathbb{M}_{1}\left(t ; \beta^{5211}, \gamma^{5211}\right)$ for $t=0,1, \ldots, 119$ (from Dec 2011 until Dec 2020 ), we can predict, probabilistically, the transmission dynamics of the carriers. Then, for each time instant $t$ we take the model output and we calculate the $95 \%$ $\mathrm{CI}$ and the mean of the expectation. The results are shown in Figure 8.2.

The probabilistic fitting technique allows us to estimate the density functions for model parameters applying the so-called kernel technique to the 5211 samples of parameters $\beta, \gamma$ obtained [84, Chapter 8]. These density functions can be seen in Figure 8.3.

Now, let us observe Figure 8.3. This plot gives us an idea about the time an individual needs to clear meningococcus bacteria looking at parameter $\gamma$. The $95 \%$ confidence interval of $\gamma$ is $[0.1007,0.7895]$, and taking into account that $1 / \gamma$ is the average time to clear the meningococcus, we have that the estimated time needed to clear any meningococcus bacterium is $[1 / 0.7895,1 / 0.1007]=[1.26662,9.93049]$ 
Chapter 8. Studying the evolution over the next few years of the meningococcal genogroup $W$ carriers in Spain

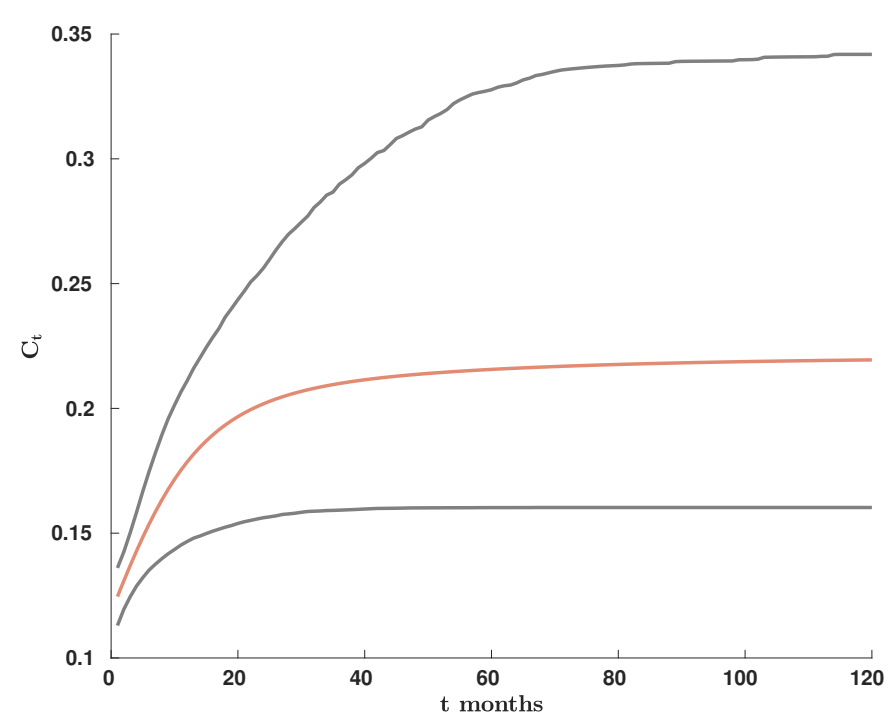

Figure 8.2: $95 \%$ CI of the solution SP of the percentage of carriers given by model (8.2). The gray lines represents the percentiles 2.5 and 97.5 and the red one, the mean, for each month, $t$, from Dec 2011 until Dec 2021.

months with $95 \%$ CI. These figures are in accordance with the ones in the extant literature [112].

We can perform similar calculations for the Lotka-Volterra model $\mathbb{M}_{2}\left(t ; \alpha, N_{1}\right)$. In this case, we have obtained $m_{k}=651$ with $N=10000$.

Notice that the order of the fractional derivative, $\alpha$ is far from 1 (that corresponds to the classical derivative) as we can see in Figure 8.5. Specifically, the mean of the PDF of $\alpha$ shown in this plot is 0.371326 . Its $95 \%$ CI is [0.004352, 0.7383]. This supports the idea of the necessity of using fractional derivatives against classical derivatives in order to explain properly the competitiveness dynamics among the different genogroups of meningococci.

Finally, the product of both solution SPs, $C_{t}$ and $x_{t}$, of models (8.2) and (8.13), respectively, will give us the percentage of Men W-135 carriers among all the population. In a personal communication, Dr. Julio Vázquez, from the Neisserias Reference Laboratory for Meningococcus of the Spanish Institute of Health Carlos 


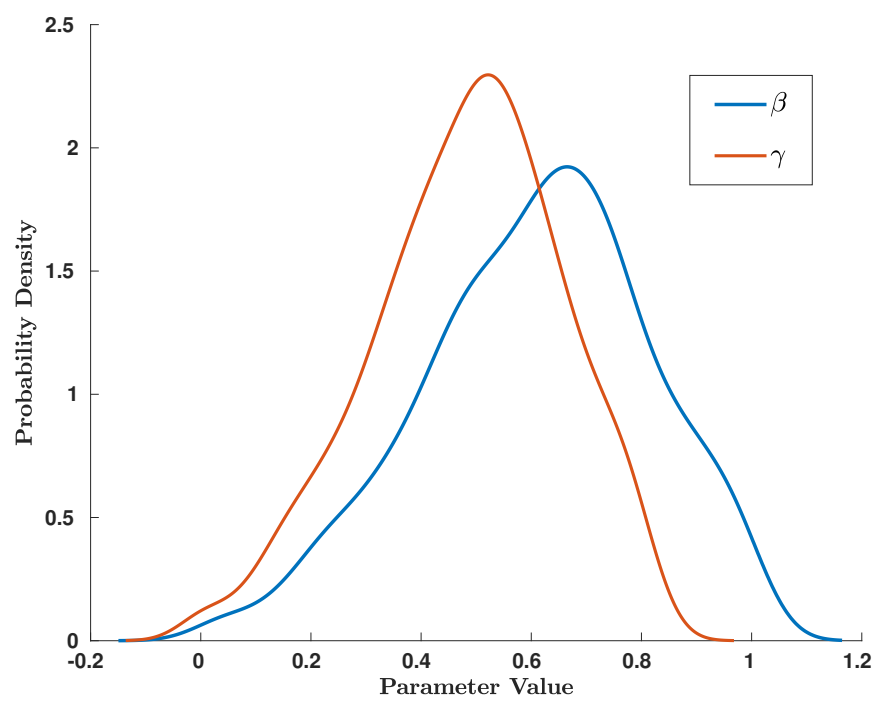

Figure 8.3: Probability density functions for the parameters $\beta$ (blue line) and $\gamma$ (red line) of model (8.2). Notice that there are non-zero probabilities for negative values of these parameters, although in any reasonable epidemiological model they are considered positive. This feature is a consequence of the kernel technique and these negative unrealistic values can be overlooked. 
Chapter 8. Studying the evolution over the next few years of the meningococcal genogroup $W$ carriers in Spain

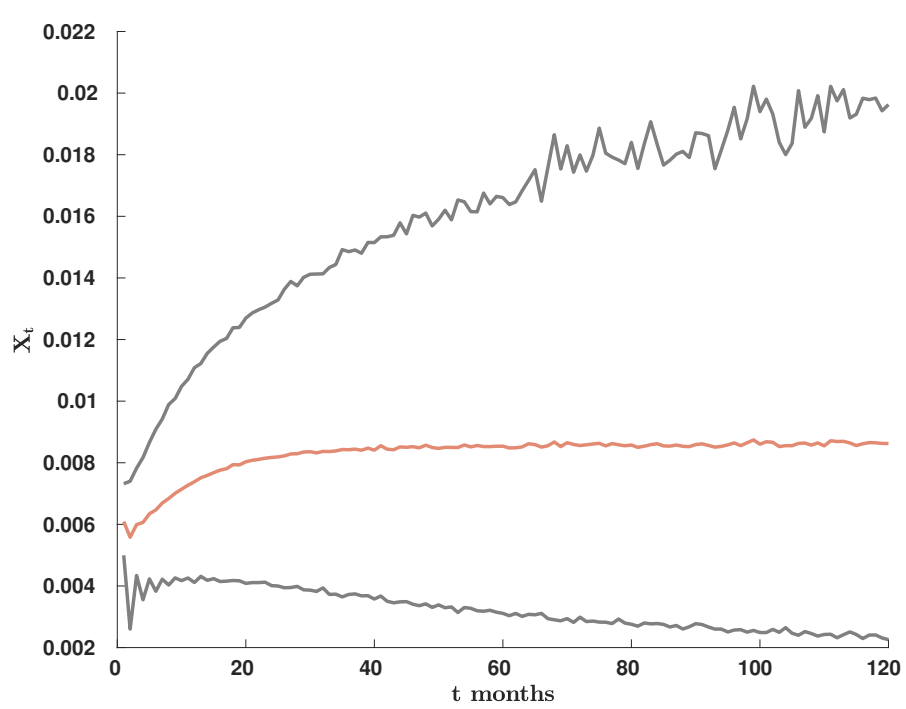

Figure 8.4: Confidence intervals of the solution SP for the percentage of meningococcus W-135 computed by the numerical scheme given in Eq. (8.13). The gray lines represents the percentiles 2.5 and 97.5 and the red line, the mean, for each time, $t$, from Dec 2011 until Dec 2020 .

III, told us that a percentage of $4 \%-5 \%$ or greater of carriers of Men W-135 in Spain would begin to be concerning.

Finally, taking into account the probabilistic information obtained previously for all time instants $t$, we are able to calculate the probability that the percentage of carriers of Men W-135 be greater than 5\%. In Table 8.6 we can see the obtained results. In Figure 8.6 a graph with the evolution of the probabilities of having $5 \%$ of carriers of Men W-135 or more from Dec 2011 until Dec 2021. As we can see, the probability of having worrying percentages of carriers of Men W-135 is very low and, then, an outbreak is not expected.

\begin{tabular}{|c|c|}
\hline Date & Probability W-135 $\geq 5 \%$ \\
\hline December 2017 & $2 \times 10^{-4}$ \\
December 2018 & 0.0014 \\
December 2019 & 0.0015 \\
December 2020 & 0.0018 \\
\hline
\end{tabular}

Table 8.6: Probability that the percentage of carriers of Men W-135 be greater than $5 \%$. 


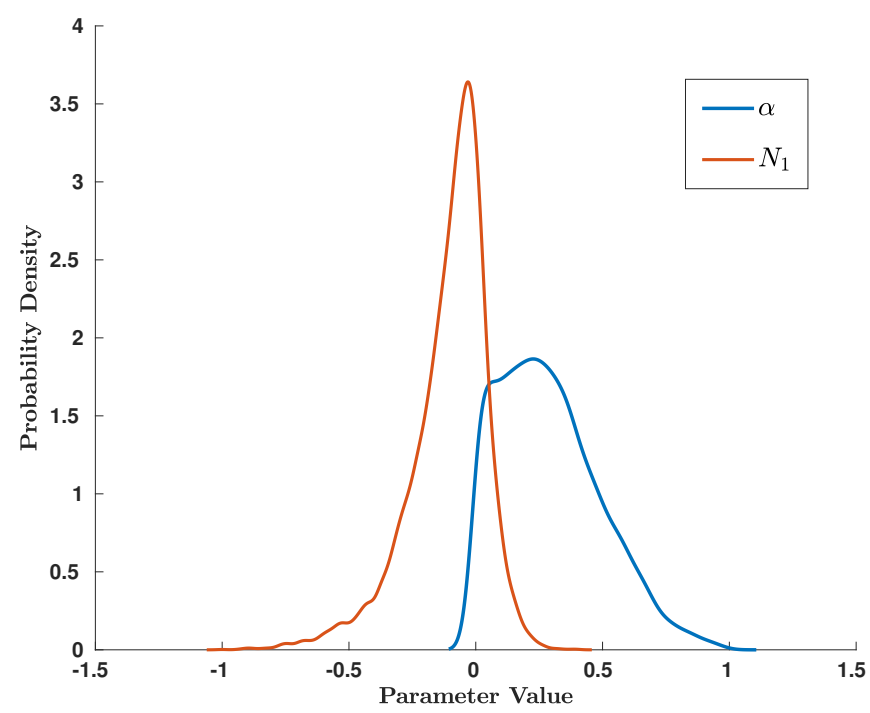

Figure 8.5: PDFs for the parameters of the Lotka-Volterra model. The red line corresponds to the parameter $N_{1}$ and the blue line to the fractional derivative order, $\alpha$. The short piece of negative values of the $\alpha$ parameter that appears in the plot, is a feature of the application of the so-called kernel technique used to construct the PDFs. This piece must been neglected since $0<\alpha<1$.

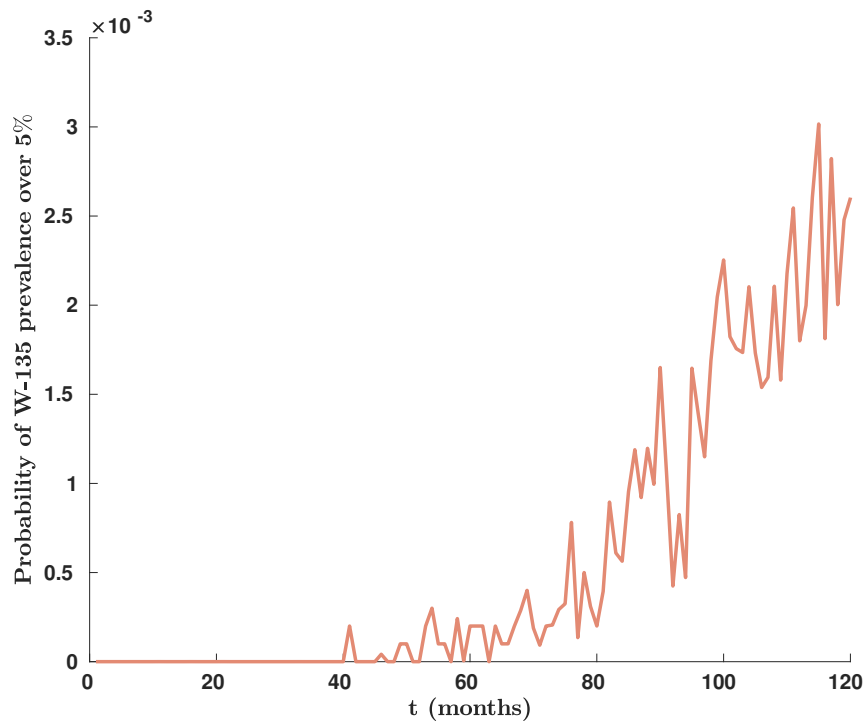

Figure 8.6: Probability of having $5 \%$ of carriers of Men W-135 or more in Spain. Prediction from Dec 2011 until Dec 2021. 
Chapter 8. Studying the evolution over the next few years of the meningococcal genogroup $W$ carriers in Spain

\subsection{Conclusions}

In this chapter we have proposed a probabilistic model for the complex ecosystem composed by several meningococcal genogroups and the human hosts carriers. The competition among the different genogroups has been discussed in the framework of a Lotka-Volterra model with fractional derivatives. The Lotka-Volterra system is a well-known tool for simulating different population of the same species competing for the same resources and it has been applied in ecology and evolutionary models. Our model is generalized taking advantage of fractional calculus in order to consider the effects of the memory of this competition in the form of genetic changes of the strains.

The interaction with the human hosts is described in terms of a discrete susceptibleinfectious-susceptible model in which the infectious are called carriers. This SCS model involves two parameters corresponding to the average time of recovery from the carrier state, $1 / \gamma$, and the infective rate, $\beta$. We must also notice that meningococcal disease is very difficult to analyse through seroepidemiological studies because of the low carriage at a given time and the continuous mutation and recombination of DNA in the strains. For that reason, only a few mathematical models have been considered and, in particular, very little is known about the epidemiology of the W-135 genogroup which has caused recent outbreaks in Chile and the United Kingdom. Immigration from Africa is thought to be the main cause of the emergence of these strains in America and Europe where they were almost non-existent or very rare even a decade ago. This is a reason of major concern for Public Health policies because of the high mortality rate for cases of invasive $\mathrm{W}-135$ meningococcal disease.

In Spain there have been two seroepidemiological studies in 2011 and 2012 in which the W-135 genogroup was found in a small percentage of individuals. By fitting the results using a probabilistic approach, we have been able to determine the probability densities for the parameters of the Lotka-Volterra and SCS model. On the other hand, the prediction for the increase of W-135 in the near future shows that the probability for the carrier population to surpass the critical threshold of $5 \%$ is below a $0.3 \%$ and so we can fairly say that an outbreak of W-135 in Spain, under the present population and immigration conditions, is negligible. On the 
other hand, our model does not take into account an increase of W-135 carrier proportion in Africa, UK or South America and the corresponding risk of further contact of Spanish population with some of these carriers who could arrive to Spain.

Finally, we want to point out that, the effects of outbreaks in other countries in the present globalized world which takes into account immigration rates and the possibility of increased arrival of W-135 carriers as well as their contact with the native population is a problem that should be addressed in a future extended model to be discussed elsewhere.

\section{Chapter published}

The results of this chapter have been published in [2]. With regard to this paper, the $\mathrm{PhD}$ candidate has contributed by working in its complete development with more emphasis on the inclusion fractional operators in the model, the developement and implementation of the random computational approach and participating in the writing of the paper. 



\section{Chapter}

\section{Modeling breast tumor growth by a}

\section{randomized logistic model: A computational approach to treat uncertainties via probability densities}

In Chapter 8, a computational approach to describe probabilistically the outbreaks of meningococcus $W$-315 infections in Spain has been developed. A confidence interval representing the carriers of meningococcus $W$-315 has been obtained by combining a SCS model with a fractional Lotka-Volterra model. Nevertheless, more statistical information is occasionally required to quantify the uncertainty of a biological process. The computation of fractional derivatives requires high computational cost and this sometimes limits the development of new computational techniques to quantify exhaustively the uncertainty. In this chapter, we set random fractional calculus to focus on describing a theoretical-computational approach in order to seek suitable random inputs (initial condition and coefficients) of the randomized discrete Pielou logistic model. In this way, we will search suitable probability density functions for random inputs so that they fit the ones assigned to breast tumour volume data at different time 
instants. The approach seems to be flexible enough to be adapted to random fractional models in future works.

\subsection{Introduction}

Breast cancer is one of the most common malignant diseases in the female population, around $1 / 8$ of women are affected by this illness. It is the second most commonly diagnosed cancer in women worldwide, [50,68]. Over the last decades, this type of cancer is increasing due to several reasons: the enlargement of the life expectancy and, consequently, the increase of DNA mutations, and the current unhealthy lifestyle (physical inactivity, obesity, living in polluted areas, etc.). The breast cancer is the first cause of death by malignant tumours in the female population aged between 40 and 59. Nevertheless, in the recent years, the survival of this malignancy has been increased because of new therapies and the early prevention and prediction [12].

A key point in the early prevention of breast cancer is the capacity of measure the volume of tumours and predict their growth over the time. To quantify the volume of tumours, doctors use approximate measurement techniques based on medical images via electronic devices, $[17,74]$. These measurements involve intrinsic errors in the real volume dimension that must be taken into account. As it shall be seen later, errors can be modelled by applying the principle of maximum entropy (PME) described in Section 6.4, that allows us to allocate reliable uncertainties to sampled tumour volume data [91].

To study and predict the growth of volume tumours cancer several non-linear mathematical models, based on difference and differential equations, have been successfully proposed. In [29], the authors develop a non-linear system of difference equations to study the short term dynamics of the bladder cancer and the immune response of patients. In [90] a numerical scheme for solving time-fractional cancer invasion system with non-local diffusion has been recently proposed. In this chapter, authors propose an optimal control strategy to enhance the power of NK-cells and Effector T-cells in order to more quickly eradicate the cancer. In [38], authors perform a numerical analysis to understand the dynamics of cancer invasion using a time-fractional system. In [16], the classical Gomperzian growth is applied to 
study the breast tumour volume before applying suitable therapies. In [11], the logistic model is parametrized to predict the treatment response and changes in breast cancer cellularity during neoadjuvant chemotherapy. Cancer development is a process where normal somatic cells acquire mutations, in [54], a system of nonlinear differential equations to study the dynamics of these cells mutations is proposed. Recently, the dynamics of a cell line (MCF-7) in human breast cancer has been described using the same type of mathematical formulation, [68].

As it has been previously pointed out, tumour volume data involves uncertainties, then it is natural to consider random models to study the evolution over time. In this chapter, we consider a randomized logistic-type model to study the growth of breast tumour volume. The initial condition and coefficients are considered RVs whose probability distributions must be consistently set from sampled information. Despite the simple formulation of the logistic model, it has demonstrated to be very effective to describe the dynamics of biological growth processes like tumours $[12]$.

Indeed, in dealing with practical applications of random models to describe breast tumours volume using real data, a main challenge is allocating appropriate probability distributions for model parameters so that the output model, which is a $\mathrm{SP}$, satisfactorily captures data uncertainties. In this chapter we face this key challenge by developing a computational technique to quantify uncertainties and then performing more realistic predictions to modelling breast tumours volume by means of a random logistic equation using real data. Assuming randomness to each model parameter (initial condition and coefficients), this computational technique is based on seeking the best PDF of model parameters so that the PDF of the solution SP of the random logistic model matches the PDF assigned, via PME, to sampled data of breast tumor volume at each time instant. In this manner, through the PDF, we perform a more complete probabilistic description of the breast tumour volume dynamics than constructing predictions based only on the expectation and confidence intervals.

At this point, it is important to emphasize that when applying random models to describe real problems, the allocation of appropriate probability distributions to model parameters is often done using heuristic arguments based on positiveness, 
boundedness and/or meta-information [34, 33]. This limits the choice of distributions to particular families. For instance, for positive and bounded parameters, the Beta distribution may be an appropriate candidate; for positive and unbounded parameters, the Gamma distribution might be suitable; etc. In contrast, the PME method allows us to give more flexibility when assigning probability distributions to each model parameters, since a parametric family of distributions are seeking for.

Our analysis will be presented in the following steps. In Section 9.2, the randomized discrete logistic model is presented together with the expression of the PDF of its solution SP. In Section 9.3 we will apply the PME, described in Section 6.4, to assign an explicit PDF to each sampled data. In Section 9.4 we will again utilize the PME to represent the PDF of each model input via closed expressions, which depend on certain parameters to be determined later. In Section 9.5 we design a computational procedure to determine the aforementioned parameters so that the density of the model solution be as close as possible to the density previously allocated to sampled data. In Section 9.6 we will apply the computational approach, introduced in the previous section, to first obtain the densities of model inputs and, secondly of the model output. Finally, conclusions are drawn in Section 9.7.

\subsection{A randomized discrete logistic model}

The logistic model has been extensively applied to describe the dynamics of growth processes in different scientific areas, as pharmacology [55], epidemiology [18] or ecology [107]. In this chapter we are interested in its application in medicine to model tumor growth $[11,113]$.

In this chapter we consider the following discrete dynamical system, usually referred to as the Pielou model [103, 108],

$$
X_{n+1}=\frac{A X_{n}}{1+B X_{n}}, \quad n=0,1,2, \ldots,
$$


for a given initial condition $X_{0}$. As it can be seen in [103], this discrete model comes from the classical Verhulst continuous logistic equation [118]

$$
V^{\prime}(t)=a V(t)\left(1-\frac{V(t)}{b}\right)
$$

being $a>0$ the growth rate and $b>0$ the carrying capacity. According to [103, pages 19 - 22], models (9.1) and (9.2) are related via the following relationship of their respective parameters,

$$
A=e^{a}, \quad B=\frac{e^{a}-1}{b} .
$$

Since $a>0$ and $b>0$, then $A>1$ and $B>0$.

As it has been pointed out in the foregoing section, uncertainty quantification is a main goal in modelling breast tumours growth from real data. This aim us at treating the parameters $A, B$ and $X_{0}$ in model (9.1) as RVs belonging to a complete probability space $(\Omega, \mathcal{F}, \mathbb{P})$. As a consequence, model parameters depend on outcomes $\omega \in \Omega$, i.e., $A=A(\omega), B=B(\omega)$ and $X_{0}=X_{0}(\omega)$, and then the solution is a SP, $X_{n}=X_{n}(\omega)$. As usual, hereinafter the $\omega$-notation will be hidden.

The properties random quantities are often described via statistical moments. However, it is more desirable to do it through probability distributions. Specifically, fixed t, from the so called first PDF, $f_{Y}(y, t):=f_{Y}(y)$, of a SP, say $Y(t)$, one can calculate one-dimensional statistical moments of arbitrary order and the probability that the process lies in a specific interval of interest equations (4.1) and (4.2), respectively.

By applying the random transformation technique [109], recently some of the authors have obtained an explicit expression of the first PDF, $f_{X_{n}}$, to the solution of the randomized Pielou model (9.1), [43]. Specifically, by assuming that $A, B$ and $X_{0}$ are absolutely continuous RVs with a joint PDF, $f_{X_{0}, A, B}$, they obtained

$$
\begin{aligned}
f_{X_{n}}(x)= & \int_{\mathcal{D}(A, B)} f_{X_{0}, A, B}\left(\frac{x(a-1)}{a^{n}(a-1)-b x\left(a^{n}-1\right)}, a, b\right) \\
& \cdot\left|\frac{(a-1)^{2} a^{n}}{\left(a^{n}(a-1)-b x\left(a^{n}-1\right)\right)^{2}}\right| \mathrm{d} a \mathrm{~d} b,
\end{aligned}
$$


where $\mathcal{D}(A, B)$ denotes the domain of random vector $(A, B)$, [43]. In the particular case that $A, B$ and $X_{0}$ are independent, then $f_{X_{0}, A, B}\left(x_{0}, a, b\right)=f_{X_{0}}\left(x_{0}\right) f_{A}(a) f_{B}(b)$ (being $\mathrm{f}_{X_{0}}, f_{A}$ and $f_{B}$ the PDF of $X_{0}, A$ and $B$, respectively) and, as a consequence, the PDF of the solution can be represented as

$$
\begin{gathered}
f_{X_{n}}(x)=\int_{\mathcal{D}(A, B)} f_{X_{0}}\left(\frac{x(a-1)}{a^{n}(a-1)-b x\left(a^{n}-1\right)}\right) f_{A}(a) f_{B}(b) \\
\cdot\left(\frac{(a-1)^{2} a^{n}}{\left(a^{n}(a-1)-b x\left(a^{n}-1\right)\right)^{2}}\right) \mathrm{d} a \mathrm{~d} b .
\end{gathered}
$$

Computing this double integral in an exact way, i.e. using primitives, is not always possible. Nevertheless, using numerical quadrature rules we can approximate it. This fact mainly depends upon the mathematical expression of the densities $f_{X_{0}}, f_{A}$ and $f_{B}$. To overcome this drawback, we will consider the following representation of $f_{X_{n}}$ in terms of the expectation operator, $\mathbb{E}[\cdot]$,

$$
f_{X_{n}}(x)=\mathbb{E}\left[f_{X_{0}}\left(\frac{x(A-1)}{A^{n}(A-1)-B x\left(A^{n}-1\right)}\right)\left|\frac{(A-1)^{2} A^{n}}{\left(A^{n}(A-1)-B x\left(A^{n}-1\right)\right)^{2}}\right|\right] .
$$

At this point, it is important to underline that we can weak the condition that input parameters $A$ and $B$ are absolutely continuous RVs but just having probability distributions. Then, the density $f_{X_{n}}$ can be computed using Monte Carlo simulations. However, notice that to follow this strategy, we need to assign reliable distributions to RVs $A, B$ and $X_{0}$. This key point will be addressed using the PME described in Section 6.4

\subsection{Data and their uncertainty}

As it has been previously indicated, in this section we will apply the PME, described in Section 6.4, to assign probability distributions to each sampled data. To this end, we are going to use the following information. First, the figures tabulated in the second column of Table 9.1, that correspond to the sampled data of the breast tumour volume measured in $\mathrm{mm}^{3}$, at different days, $\tilde{n},[123$, Figure 1]. They have 
been obtained using xenograft technique, which consists of inserting cell tissue from one species to another, in our case, breast tumoral tissue from human species to a rodent species, [123, page 2]. These values have been collected by measurement electronic devices, hence involving uncertainties. This fact aims us at treating these quantities as RVs rather than deterministic values. The figures $\tilde{m}_{1, \tilde{n}}$ are taken as representing the mean and, according to [37], we assign a variance of $5 \%$ at each value, i.e. $\tilde{\sigma}_{\tilde{n}}^{2}=0.05 \tilde{m}_{1, \tilde{n}}$ (see third column, $\tilde{\sigma}_{\tilde{n}}^{2}$, Table 9.1). As a consequence, the second moment can be straightforwardly computed, $\tilde{m}_{2, \tilde{n}}=\tilde{m}_{1, \tilde{n}}^{2}+\tilde{\sigma}_{\tilde{n}}^{2}$, see last column of Table 9.1.

\begin{tabular}{|c|c|c|c|}
\hline Days & Mean $\left(\tilde{m}_{1, \tilde{n}}\right)$ & Variance $\left(\tilde{\sigma}_{\tilde{n}}^{2}\right)$ & 2nd moment $\left(\tilde{m}_{2, \tilde{n}}\right)$ \\
\hline$\tilde{n}=0$ & 45.74 & 2.287 & 2094.4 \\
$\tilde{n}=16$ & 194.257 & 9.7129 & 37745.49 \\
$\tilde{n}=30$ & 675.14 & 38.2570 & 455852.27 \\
$\tilde{n}=33$ & 936.53 & 46.8256 & 877135.26 \\
$\tilde{n}=43$ & 1941.7 & 97.0850 & 3770295.97 \\
$\tilde{n}=48$ & 2558.6 & 127.930 & 6546561.89 \\
\hline
\end{tabular}

Table 9.1: Volume of breast tumor cells using xenograft technique at different days, [123] $\left(\tilde{m}_{1, \tilde{n}}\right)$ together with the assigned variance $\left(\tilde{\sigma}_{\tilde{n}}^{2}\right)$ and second moment $\left(\tilde{m}_{2, \tilde{n}}\right)$.

Since we have information of the two first moments, we allocate the PDF of each the corresponding volume of breast tumour cell using expression (6.22), i.e.

$$
\tilde{f}_{\tilde{n}}(x)=\mathbb{1}_{\mathcal{D}(\tilde{n})} \mathrm{e}^{-1-\lambda_{0}^{\tilde{n}}-\lambda_{1}^{\tilde{n}} x-\lambda_{2}^{\tilde{n}} x^{2}},
$$

where $\mathcal{D}(\tilde{n})$ denotes the domain of the RV inferred by the information collected in Table 9.1 and $\lambda_{k}^{\tilde{n}}, k=0,1,2$, are determined solving the following system of non-linear equations for each $\tilde{n} \in\{0,16,30,33,43,48\}$, see Section 6.4 ,

$$
\begin{aligned}
& \int_{0}^{\infty} \mathrm{e}^{-1-\lambda_{0}^{\tilde{n}}-\lambda_{1}^{\tilde{n}} x-\lambda_{2}^{\tilde{n}} x^{2}} \mathrm{~d} x=1, \\
& \int_{0}^{\infty} x \mathrm{e}^{-1-\lambda_{0}^{\tilde{n}}-\lambda_{1}^{\tilde{n}} x-\lambda_{2}^{\tilde{n}} x^{2}} \mathrm{~d} x=\tilde{\mu}_{\tilde{n}}, \\
& \int_{0}^{\infty} x^{2} \mathrm{e}^{-1-\lambda_{0}^{\tilde{n}}-\lambda_{1}^{\tilde{n}} x-\lambda_{2}^{\tilde{n}} x^{2}} \mathrm{~d} x=\tilde{\mu}_{\tilde{n}}^{2}+\tilde{\sigma}_{\tilde{n}}^{2} .
\end{aligned}
$$


The results are shown in Table 9.2. They have obtained by fsolve function in MATLAB, [71].

\begin{tabular}{|c|c|c|c|}
\hline Days & $\lambda_{0}^{\tilde{n}}$ & $\lambda_{1}^{\tilde{n}}$ & $\lambda_{2}^{\tilde{n}}$ \\
\hline$\tilde{n}=0$ & 74.5841 & -3.2063 & $3.50 \mathrm{e}-02$ \\
$\tilde{n}=16$ & 21.7070 & -0.1882 & $4.8407 \mathrm{e}-04$ \\
$\tilde{n}=30$ & 10.5921 & -0.0133 & $8.6701 \mathrm{e}-06$ \\
$\tilde{n}=33$ & 11.8918 & -0.0145 & $8.0943 \mathrm{e}-06$ \\
$\tilde{n}=43$ & 9.5029 & -0.0034 & $9.3958 \mathrm{e}-07$ \\
$\tilde{n}=48$ & 8.8842 & -0.0015 & $3.1900 \mathrm{e}-07$ \\
\hline
\end{tabular}

Table 9.2: Values of $\lambda_{0}^{\tilde{n}}, \lambda_{1}^{\tilde{n}}$ and $\lambda_{2}^{\tilde{n}}$ obtained solving the system of nonlinear equations given in (9.8) the different values of $\tilde{n}$.

In Fig 9.1, we show a graphical representation of each PDF given by equation (9.7) with the values collected in Table 9.2. We can observe the PDFs built via the PME provide higher variability as $\hat{n}$ increases in full agreement with the variance $\tilde{\sigma}_{\tilde{n}}^{2}$ given in Table 9.1 . 

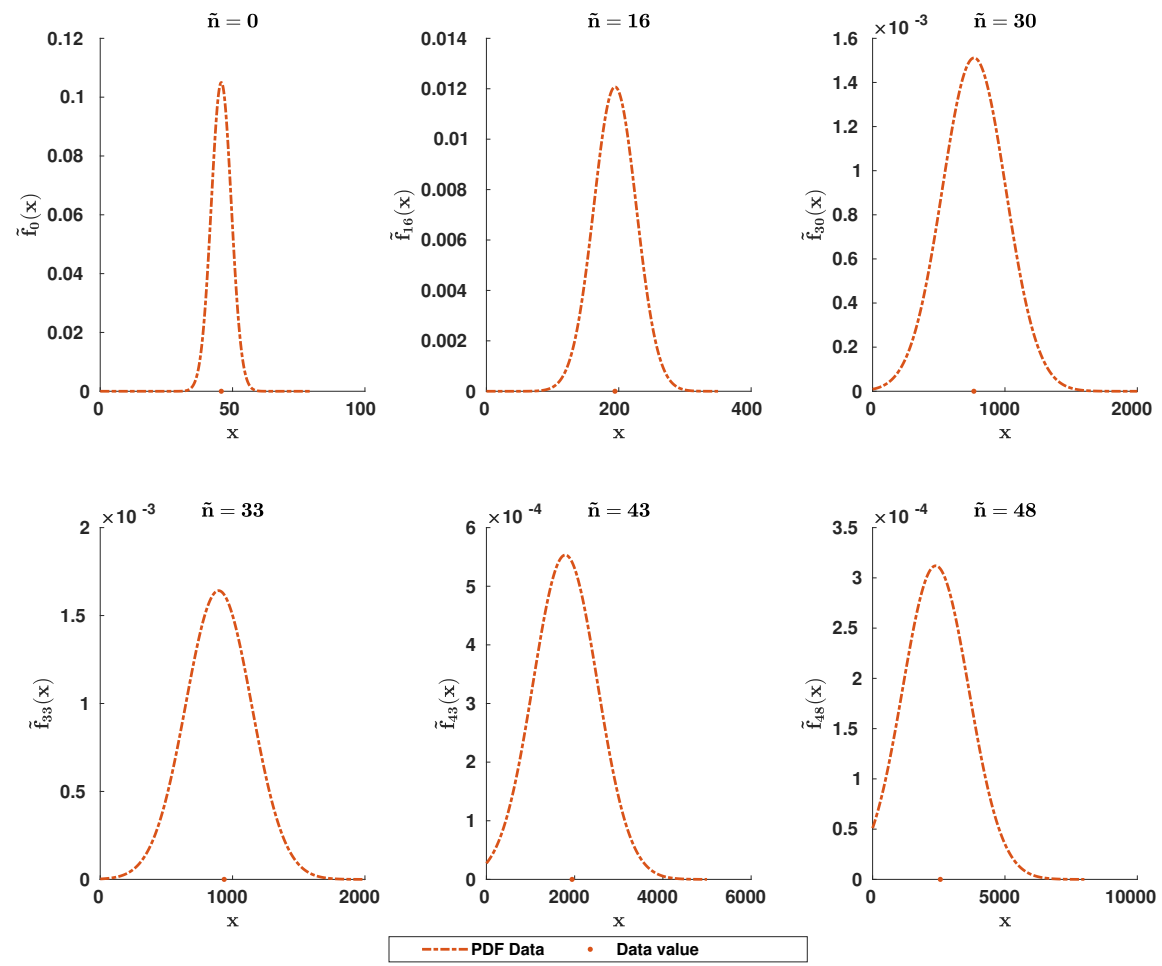

Figure 9.1: PDF of each sampled data using the PME at the days $\tilde{n} \in\{0,16,30,33,43,48\}$. The red points represent the values $\tilde{m}_{1, \tilde{n}}$ given in Table 9.1. 


\subsection{Statistical distribution of the model parameters}

Once probability distributions to sampled data have been assigned, as it has been indicated in the Introduction section, the following step will consist of establishing probability distributions for model parameters $A, B$ and $X_{0}$. To achieve this goal, the PME will be applied again.

For consistency with the distributions assigned in Section 9.3 for the first sampled data, corresponding to $\tilde{n}=0$, we take

$$
f_{X_{0}}\left(x_{0}\right)=\mathrm{e}^{-1-\lambda_{0}^{0}-\lambda_{1}^{0} x_{0}-\lambda_{2}^{0} x_{0}^{2}},
$$

where $\lambda_{0}^{2}=74.5841, \lambda_{1}^{0}=-3.2063$ and $\lambda_{2}^{0}=3.50 e-02$, see first row in Table 9.2. Using PME, we propose the following parametric PDFs for the rest of RVs $A$ and $B$

$$
\begin{array}{cc}
f_{A}(a)=\mathrm{e}^{-1-\lambda_{0}^{A}-\lambda_{1}^{A} a-\lambda_{2}^{A} a^{2}}, & a \in\left[a_{1}, a_{2}\right], \\
f_{B}(b)=\mathrm{e}^{-1-\lambda_{0}^{B}-\lambda_{1}^{B} b-\lambda_{2}^{B} b^{2}}, & b \in\left[b_{1}, b_{2}\right],
\end{array}
$$

respectively. According to (9.3), we derive that $a_{1}=1$ and $b_{1}=0$, hence $a_{2}>1$ and $b_{2}>0$.

The values of parameters $\left\{\lambda_{0}^{A}, \lambda_{1}^{A}, \lambda_{2}^{A}\right\}$ and $\left\{\lambda_{0}^{B}, \lambda_{1}^{B}, \lambda_{2}^{B}\right\}$ must be chosen so that $f_{A}$ and $f_{B}$ integrate the unit. Therefore, after calculating the integral and isolating $\lambda_{0}^{A}$ and $\lambda_{0}^{B}$ one gets, respectively,

$$
\lambda_{0}^{A}=-1+\frac{\left(\lambda_{1}^{A}\right)^{2}}{4 \lambda_{2}^{A}}+\log \left[\frac{\sqrt{\pi}}{2 \sqrt{\lambda_{2}^{A}}}\left(\operatorname{Erf}\left(\frac{\lambda_{1}^{A}+2 a_{2} \lambda_{2}^{A}}{2 \sqrt{\lambda_{2}^{A}}}\right)-\operatorname{Erf}\left(\frac{\lambda_{1}^{A}+2 a_{1} \lambda_{2}^{A}}{2 \sqrt{\lambda_{2}^{A}}}\right)\right)\right],
$$




$$
\lambda_{0}^{B}=-1+\frac{\left(\lambda_{1}^{B}\right)^{2}}{4 \lambda_{2}^{B}}+\log \left[\frac{\sqrt{\pi}}{2 \sqrt{\lambda_{2}^{B}}}\left(\operatorname{Erf}\left(\frac{\lambda_{1}^{B}+2 b_{2} \lambda_{2}^{B}}{2 \sqrt{\lambda_{2}^{B}}}\right)-\operatorname{Erf}\left(\frac{\lambda_{1}^{B}+2 b_{1} \lambda_{2}^{B}}{2 \sqrt{\lambda_{2}^{B}}}\right)\right)\right],
$$

provided $\lambda_{2}^{A}>0$ and $\lambda_{2}^{B}>0$. Here, $\operatorname{Erf}(x)=\frac{2}{\sqrt{\pi}} \int_{0}^{x} e^{-t^{2}} \mathrm{~d} t$ stands for the error function.

According to expression (9.6), to compute the PDF of the solution SP $X_{n}$, it is necessary to sampling RVs $A$ and $B$. This will be done via the inverse of the distribution functions of $A$ and $B$ using the so called inverse transformation method, [51, Chapter 2]. According to this technique, we first need to calculate the distribution functions of $A$ and $B$,

$$
\begin{aligned}
F_{A}(a) & =\int_{1}^{a} f_{A}(s) \mathrm{d} s \\
& =\frac{1}{2 \sqrt{\lambda_{2}^{A}}} \mathrm{e}^{-1-\lambda_{0}^{A}+\frac{\left(\lambda_{1}^{A}\right)^{2}}{4 \lambda_{2}^{A}}} \sqrt{\pi}\left(-\operatorname{Erf}\left(\frac{\lambda_{1}^{A}+2 \lambda_{2}^{A}}{2 \sqrt{\lambda_{2}^{A}}}\right)+\operatorname{Erf}\left(\frac{\lambda_{1}^{A}+2 a \lambda_{2}^{A}}{2 \sqrt{\lambda_{2}^{A}}}\right)\right)
\end{aligned}
$$

and

$$
\begin{aligned}
F_{B}(b) & =\int_{0}^{b} f_{B}(s) \mathrm{d} s \\
& =\frac{1}{2 \sqrt{\lambda_{2}^{B}}} \mathrm{e}^{-1-\lambda_{0}^{B}+\frac{\left(\lambda_{1}^{B}\right)^{2}}{4 \lambda_{2}^{B}}} \sqrt{\pi}\left(-\operatorname{Erf}\left(\frac{\lambda_{1}^{B}}{2 \sqrt{\lambda_{2}^{B}}}\right)+\operatorname{Erf}\left(\frac{\lambda_{1}^{B}+2 b \lambda_{2}^{B}}{2 \sqrt{\lambda_{2}^{B}}}\right)\right),
\end{aligned}
$$

where $1 \leq a \leq a_{2}$ and $0 \leq b \leq b_{2}$, respectively. Denoting $u_{A}:=F_{A}(a) \in(0,1)$ and $u_{B}:=F_{B}(b) \in(0,1)$ in $(9.14)$ and $(9.15)$, respectively, and isolating $a$ and $b$ in each expression, one gets 
$a=\frac{1}{2 \lambda_{2}^{A}}\left(-\lambda_{1}^{A}+2 \sqrt{\lambda_{2}^{A}} \operatorname{InvErf}\left(\frac{2 \mathrm{e}^{1+\lambda_{0}^{A}-\frac{\left(\lambda_{1}^{A}\right)^{2}}{4 \lambda_{2}^{A}}} \sqrt{\pi} u_{A} \sqrt{\lambda_{2}^{A}}+\pi \operatorname{Erf}\left(\frac{\lambda_{1}^{A}+2 \lambda_{2}^{A}}{2 \sqrt{\lambda_{2}^{A}}}\right)}{\pi}\right)\right)$

and

$b=\frac{1}{2 \lambda_{2}^{B}}\left(-\lambda_{1}^{B}+2 \sqrt{\lambda_{2}^{B}} \operatorname{InvErf}\left(\frac{2 \mathrm{e}^{1+\lambda_{0}^{B}-\frac{\left(\lambda_{1}^{B}\right)^{2}}{4 \lambda_{2}^{B}}} \sqrt{\pi} u_{B} \sqrt{\lambda_{2}^{B}}+\pi \operatorname{Erf}\left(\frac{\lambda_{1}^{B}}{2 \sqrt{\lambda_{2}^{B}}}\right)}{\pi}\right)\right)$,

respectively. Here $\operatorname{Inv} \operatorname{Erf}(\cdot)$ denotes the inverse function of $\operatorname{Erf}(\cdot)$. Sampling many times $u_{A}$ and $u_{B}$ uniformly in the unit interval $(0,1)$, i.e. $u_{A}, u_{B} \sim U(0,1)$, and substituting these sampled values in expressions (9.16) and (9.17), we obtain simulations of RVs $A$ and $B$, respectively.

\subsection{Procedure design}

In the previous section, we have taken advantage of PME to assign reliable PDFs to model inputs $A$ and $B$ (see expressions (9.10) and (9.11), respectively). Taking into account the relations (9.12) and (9.13), these PDFs, $f_{A}$ and $f_{B}$, depend on parameters $\left\{\lambda_{1}^{A}, \lambda_{2}^{A}, a_{2}\right\}$ and $\left\{\lambda_{1}^{B}, \lambda_{2}^{B}, b_{2}\right\}$, respectively. In this section, we design a computational procedure to determine these parameters so that the PDF, $f_{X_{n}}$, which according to (9.6) depends on $A$ and $B$, matches, as much as possible, the PDFs constructed via PME in Section 9.3 of sampled data at the time instants $\tilde{n} \in\{0,16,30,33,43,48\}$.

To seek the parameters $\lambda_{1}^{A}, \lambda_{2}^{A}, a_{2}, \lambda_{1}^{B}, \lambda_{2}^{B}$ and $b_{2}$, an optimization algorithm will be applied. This technique consists of comparing, over various iterations, sets of admissible parameters $\left(\lambda_{1}^{A}, \lambda_{2}^{A}, a_{2}, \lambda_{1}^{B}, \lambda_{2}^{B}, b_{2}\right)$ until an optimum or a satisfactory set is found [121]. 
To compare sets of admissible parameters, a suitable criterion, which is enclosed in a fitness function, is required. In our case, given a set of parameters $\left(\lambda_{1}^{A}, \lambda_{2}^{A}, a_{2}, \lambda_{1}^{B}, \lambda_{2}^{B}, b_{2}\right)$, we have chosen the sum of certain local errors, $E_{\tilde{n}}$, $\tilde{n} \in\{0,16,30,33,43,48\}$, which are defined in terms of the absolute differences between the PDF, $f_{X_{\tilde{n}}}$, given in (9.6) and the PDF, $\tilde{f}_{\tilde{n}}$, assigned to sampled data given in equation (9.7) and Table 9.2.

Down below, we shall describe through several steps the construction of the fitness function, $F F(s)$, for a given set of parameters $s=\left(\lambda_{1}^{A}, \lambda_{2}^{A}, a_{2}, \lambda_{1}^{B}, \lambda_{2}^{B}, b_{2}\right)$.

Step 1: Compute the values of $\lambda_{0}^{A}$ and $\lambda_{0}^{B}$ defined by equations (9.12) and (9.13), respectively.

Step 2: Obtain $M=10000$ samples of $u_{A}, u_{B} \sim U(0,1)$ and substitute them in equations (9.16) and (9.17) to sampling values $a$ and $b$ of RVs $A$ and $B$, respectively.

Step 3: Define the mesh of $N+1$ nodes over the interval $[0, H]$,

$$
\hat{x}:=\left\{x_{i}\right\}:=\left\{\frac{i H}{N}\right\}_{i=0}^{N}
$$

being $H<+\infty$ an upper bound of the RV defined by equation (9.7) at $\tilde{n}=48$. In our application we will take $N=500$ and $H=8000$ (see panel corresponding $\tilde{n}=48$ in Figure 9.1).

Step 4: Fix $\tilde{n} \in\{0,16,30,33,43,48\}$ and $x_{i}$ defined in Step 3. Substitute the $M$ simulations $(a, b)$ of the random vector $(A, B)$ generated in Step 2 in the expectation argument of (9.6), i.e., in the expression

$$
f_{X_{0}}\left(\frac{x_{i}(a-1)}{a^{\tilde{n}}(a-1)-b x_{i}\left(a^{\tilde{n}}-1\right)}\right)\left|\frac{(a-1)^{2} a^{\tilde{n}}}{\left(a^{\tilde{n}}(a-1)-b x_{i}\left(a^{\tilde{n}}-1\right)\right)^{2}}\right|,
$$

Thus, for each $\tilde{n}, M$ curves, along the mesh $\hat{x}$, are generated.

Step 5: For each day $\tilde{n}$, compute the average of the $M$ curves generated in Step 4. Then, according to (9.6) an approximation of the PDF $f_{X_{\tilde{n}}}$ evaluated in $\hat{x}$ is obtained. 
Step 6: For each day $\tilde{n}$, evaluate in the mesh $\hat{x}$ the PDF, $\tilde{f}_{\tilde{n}}$, of sampled data defined by equation (9.7) and Table 9.2.

Step 7: For each day $\tilde{n} \in\{0,16,30,33,43,48\}$, compute the error

$$
E_{\tilde{n}}=\frac{\sum_{i=0}^{N}\left|f_{X_{\tilde{n}}}\left(x_{i}\right)-\tilde{f}_{\tilde{n}}\left(x_{i}\right)\right|}{\sum_{i=0}^{N} \tilde{f}_{\tilde{n}}\left(x_{i}\right)} .
$$

Step 8: The output of the fitness function, named fitness, is given by

$$
E=E_{0}+E_{16}+E_{30}+E_{33}+E_{43}+E_{48} .
$$

It is important to remark that $E_{0}=0$, since by construction we have taken $f_{X_{0}}=\tilde{f}_{0}$, see Section 9.3 .

Using an optimization algorithm, we can find the vector $s=\left(\lambda_{1}^{A}, \lambda_{2}^{A}, a_{2}, \lambda_{1}^{B}, \lambda_{2}^{B}, b_{2}\right)$ that minimizes the fitness $E$, i.e. a set of parameters such that $f_{X_{\tilde{n}}}$ and $\tilde{f}_{\tilde{n}}$, are close at the time instants $\tilde{n} \in\{0,16,30,33,43,48\}$.

The optimization algorithm used in this chapter to minimize $F F$ is a bioinspired algorithm named Particle Swarm Optimization (PSO). These kind of algorithms are inspired by biological behaviour of certain species. In this case, PSO represents the movement of a swarm of birds exploring new areas to find food. In each iteration all the birds of the swarm, change their position according to balance of its particular best position and the global best position of the swarm, [80].

\subsection{Results}

This section is aimed at seeking the values $\lambda_{1}^{A}, \lambda_{2}^{A}, a_{2}, \lambda_{1}^{B}, \lambda_{2}^{B}$ and $b_{2}$ that minimize the fitness function, $F F$, described in the foregoing section through Steps $1-8$. Minimizing $F F$, we guarantee that the PDF of the randomized discrete logistic model (9.6), at the time instants $\tilde{n} \in\{0,16,30,33,43,48\}$, approximates with the PDF of sampled data described in (9.7) and Table 9.2. 
As it has been explained in Section 9.5, PSO algorithm is applied to find out the best set of parameters $s=\left(\lambda_{1}^{A}, \lambda_{2}^{A}, a_{2}, \lambda_{1}^{B}, \lambda_{2}^{B} b_{2}\right)$ that minimizes $F F$. We consider a swarm made up of 200 particles (birds), and during 90 iterations, the particles change their positions. In other words, our optimization algorithm requires 90 iterations.

Using the MATLAB function particleswarm with 200 elements and 90 iterations, we proceed to find out the best set of parameters that minimizes $F F$. This procedure requires about 3 hours to reach a suitable solution with an Intel Core i7 $7700 \mathrm{HQ}$ and $16 \mathrm{~Gb}$ of RAM. The best set of parameters and their respective fitness are collected in Table 9.3. Notice that the values of $\lambda_{2}^{A}$ and $\lambda_{2}^{B}$ are positive as required in (9.12) and (9.13).

Notice that the upper bound, $b_{2}$, of the domain of the RV $B$ is close to zero, $b_{2}=9.365588 \cdot 10^{-6}$. This numerical result is in full agreement with expression (9.3) that relates the parameter $B$, appearing in the discrete logistic model (9.1), and the parameters $a$ and $b$ involved in the formulation of continuous logistic model (9.2). On the one hand, the numerator of $B$ in expression $(9.3), e^{a}-1$, is small since the $\mathrm{RV} A=e^{a}$ takes values similar to 1 . On the other hand, the denominator of $B$ in (9.3) is given by the parameter $b$ of the logistic model (9.2), defining the carrying capacity, i.e. the maximum volume the tumor can reach. From Table 9.1, we can see that the maximum sampled volume is $2558.6 \mathrm{~mm}^{3}$, and according to the trend of sampled data, it is expected the carrying capacity, $b$, will be greater than $2558.6 \mathrm{~mm}^{3}$. As a consequence, RV $B$ takes values close to zero.

\begin{tabular}{|c|c|}
\hline Parameters & Values \\
\hline$\lambda_{1}^{A}$ & -2038.1233 \\
$\lambda_{2}^{A}$ & 919.6327 \\
$a_{2}$ & 1.11057 \\
$\lambda_{1}^{B}$ & 90.5919 \\
$\lambda_{2}^{B}$ & 196.5526 \\
$b_{2}$ & $9.365588 \mathrm{e}-06$ \\
\hline \hline Fitness & 1.582584 \\
\hline
\end{tabular}

Table 9.3: Values of parameters $\lambda_{1}^{A}, \lambda_{2}^{A}, a_{2}, \lambda_{1}^{B}, \lambda_{2}^{B}, b_{2}$ that minimize the fitness function FF using Particle Swarm Optimization algorithm. 

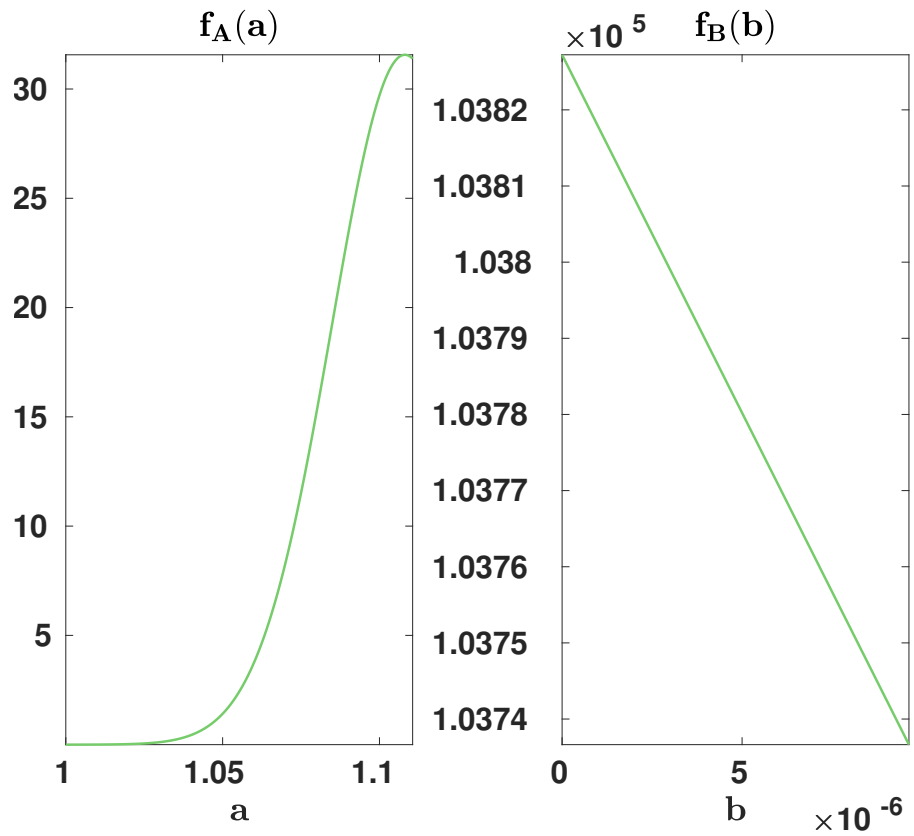

Figure 9.2: PDFs of random model parameters $A$ and $B$ of the randomized discrete logistic model described by equations (9.10) and (9.11), respectively.

A graphical representation of the PDFs of RVs $A$ and $B$ described by expressions (9.10) and (9.11), respectively, are plotted in Figure 9.2.

To better compare the obtained results, in Figure 9.3 we show, at the days $\tilde{n} \in\{0,16,30,33,43,48\}$, the PDF of the randomized discrete logistic model described in equation (9.6) (blue lines) and the PDF of sampled data described in expression (9.7) and Table 9.2 (red dashed lines). We can observe that there is a good agreement between both PDFs at every value of $\tilde{n}$. This confirms the goodness of the fitting procedure.

In Figure 9.4, we have plotted the PDF of the randomized discrete logistic model given by equation (9.6) for $n \in\{0,1, \ldots 50\}$. The red points represent the sampled data (given in column $\tilde{m}_{1, \tilde{n}}$ of Table 9.1) and green points are the means or expectations obtained via the PDFs (blue curves). 

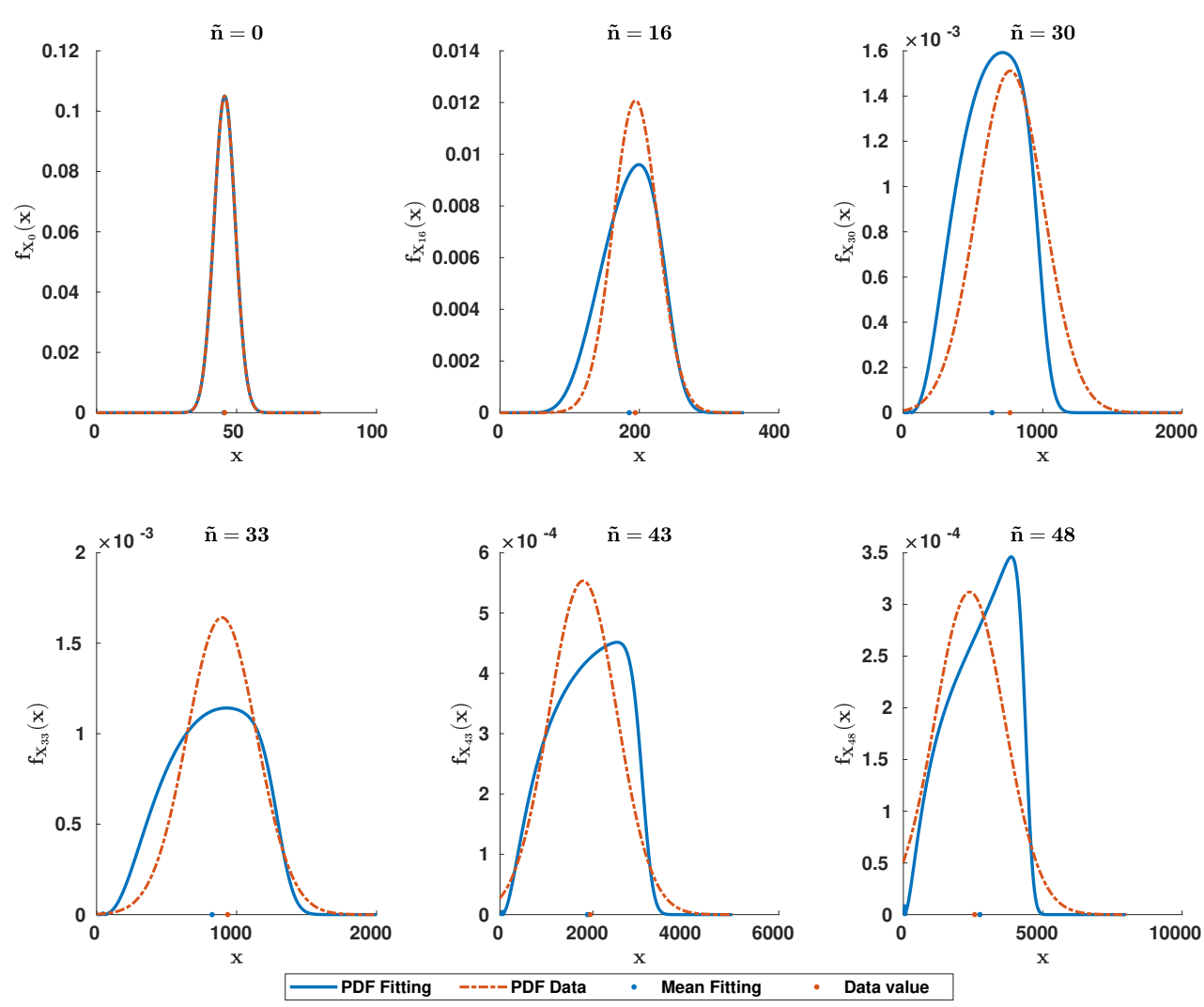

Figure 9.3: Comparison between the PDF of each sampled data (red dashed lines) and the PDF of the fitting randomized logistic model (blue lines) at the days $\tilde{n} \in\{0,16,30,33,43,48\}$. In the horizontal axis of each panel, the red point represents the sampled data (it corresponds to column $\tilde{m}_{1, \tilde{n}}$ in Table 9.1) and the blue point represents the mean or expectation obtained via the PDF (blue curves). 


\section{$\mathbf{f}_{\mathbf{X}_{\mathbf{n}}}(\mathbf{x})$}

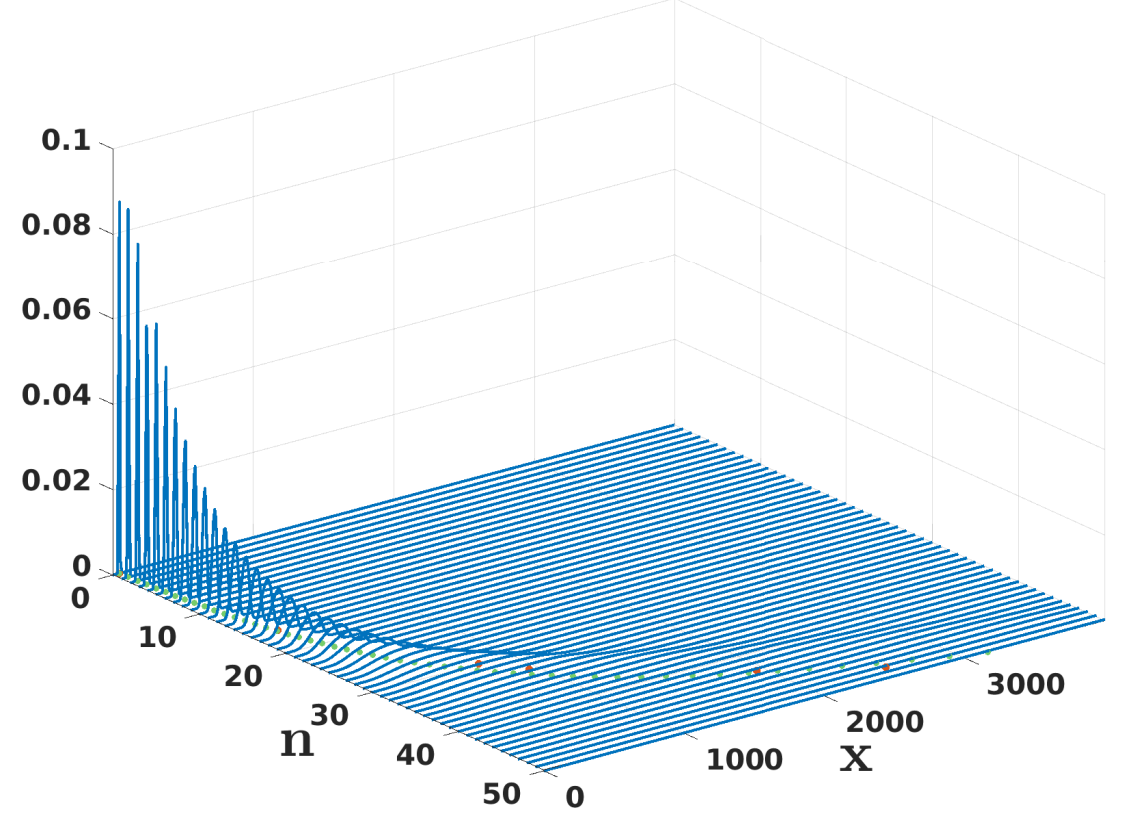

Figure 9.4: Representation of the PDF, $f_{X_{n}}$, of the random discrete logistic model (9.6) for different days $n=0,1, \ldots, 50$. Red points represent the sampled values of tumor volume described in the column $\tilde{m}_{1, \tilde{n}}$ of Table 9.1 and green points represent the mean of the distribution defined by the blue curves. 


\subsection{Conclusions}

In this chapter a probabilistic logistic-type model to describe the growth of breast tumour volume has been presented. A key aspect to treat model uncertainties has been the allocation of reliable distributions to model parameters. To handle this important issue, we have devised a computational method which takes advantage of the principle of maximum entropy. A relevant aspect of our approach is that we fit the model to real data taking into account the probabilistic information via the PDFs assigned and computed to sampled data and output model. This is a distinctive feature of our study with respect to alternative methods that perform the fitting by means of punctual statistics like the expectation. In this manner, uncertainty quantification analysis in the stochastic model is more informative. The uncertainty quantification technique proposed in this chapter may be applied to other models containing fractional derivatives where randomness in data and in model parameters play a key role $[78,61]$.

\section{Chapter published}

The results of this chapter have been published in [28]. With regard to this paper, the $\mathrm{PhD}$ candidate has contributed by working in its complete development with more emphasis on the development and implementation of the random computational procedure, the application to model breast tumour growth and participating in the writing of the paper. 



\section{Chapter}

\section{0}

A random computational procedure to recommend insulin and carbohydrates intakes

to diabetic patients

In the two previous chapters, different problems based on biological dynamics have been dealt with uncertainty. This PhD dissertation finishes with a final chapter dedicated to study an interest healthcare problem: modelling the glucose level of a diabetic patient. The aim of this chapter is making a recommendation about the insulin and the food intakes a diabetic patient needs to administrate in order to guarantee that its blood glucose level belongs to a healthy range. To do that a computational technique based on a mathematical model is proposed. The model is calibrated with glucose data one hour considering the uncertainty due to errors in the measurement of glucose. Once the model has been calibrated, proposing several combinations of insulin administration and carbohydrate ingestion, we carry out simulations with the model in order to determine which of these combinations allows the patient to remain within safe glucose levels (range levels) during the following four hours. We test our proposal using data from real patients, in three different scenarios corresponding to situations where the patient presents high, medium and low glucose levels, respectively. 


\subsection{Introduction}

Diabetes Mellitus encloses a set of metabolic disorders that affect to the level of glucose in the blood. Generally speaking, diabetes appears when the pancreas is unable to produce enough insulin (type 1) or when there is a defect in the action of the insulin (type 2) [8]. Type 1 diabetes encloses two different subtypes: subtype 1a and subtype $1 \mathrm{~b}$. Their difference is related with the performance of the immune system. In diabetes of subtype 1a, the immune system damages the tissue of beta type cells, the ones responsible to produce insulin. In subtype $1 \mathrm{~b}$ diabetes, there is not evidence that the immune system damages the beta cell types and there are periods of time where it is necessary a replacement of insulin and other periods where it is not necessary. Type 1a diabetes is commonly referred to as type 1 diabetes.

In any case, the glucose the body obtains from food is not properly transformed and there is an increasing of blood's glucose level. People suffering this kind of disorders are more likely to have cardiovascular-related illnesses among other diseases [14]. Hence, it is crucial to maintain the glucose in appropriate levels in order to avoid serious health problems. Habitually, it is said that it is convenient to maintain the glucose in the range between 70 and $180 \mathrm{mg} / \mathrm{dL}$, and to measure the quality of the management of the disease we usually speak of time in range. The higher the time in range, the better the control [19].

Several electronic devices are available nowadays to measure glucose levels over time. These devices are known as Continuous Glucose Monitoring Systems or CGMS [56]. With them, it is possible to monitor not only the evolution of the patient's glucose levels over the time, but also the actual values in the interstitial phase. Nevertheless, these devices return measurements with an intrinsic error of at least $5 \%$ that must be considered when the goal is to provide a reliable management of the patient's glucose level, reducing the possibility of suffering hyperglycemia or hypoglycemia episodes.

CGMS data analysis allow physicians to provide appropriate recommendations, specially related to adjust the protocol for injecting the insulin's doses, to do some exercise or to make changes in diet [40]. These recommendations can be more 
precise if we could predict the levels of glucose over appropriate future periods of time, and assist the medical specialist to maintain the patient's glucose levels within healthy ranges. This paper is focused on providing technical tools to improve the accuracy of the recommendations of the physician to diabetic patients.

During the last two decades several kind of models to study the dynamics of glucose have been proposed. Many recent contributions provide simulation models for enhancing the quality of live of the patients and the health care systems [98]. In [5] the authors propose a complex model to study the regulation of mechanism of glucose by pancreas's hormones in the human body. They enclose the thirteen models that mathematically clarify the mechanism of components of the blood glucose levels regulation in the human body in three main subsystems: glucose subsystem, glucagon subsystem and insulin subsystem.

The physiological parameters involved in the endogenous glucose production are constantly being researched and modeled by mathematical equations. In [111], the authors describe a system of partial differential equations to describe de endogenous glucose production dynamics.

The main disadvantage of the above two models is the complexity of the equations involved in their description. Implementing and executing problems described by complex equations may need a high computational cost in calibration and simulation, what may take longer time than the prediction time.

On the other hand, minimal models are parsimonious descriptions, which allow the measure of the processes involved in the metabolism of glucose [120]. The advantage is that they use a reduced number of parameters and the equations are computationally easy to implement and simulate. Here, we use the minimal model presented in [1] which is an adaptation of the model by Prud'homme and Bock $[106,15]$. This model has been developed with the main aim at achieving control of postprandial levels of glucose. We select this model because it uses therapeutic parameters that are usually present in the daily clinical practice. However, the model is non-linear and contains several unknown parameters related to the absorption of the insulin and its sensitivity, the production of the endogenous 
glucose, the bioavailability of the meal and the effectiveness at zero insulin. The values of the parameters of the model can be identified for each patient [1].

In this paper, we propose a computational procedure, for selecting the appropriate combination of insulin doses-food intakes-times in different situations of the daily life of a diabetic patient taking into account the inherent uncertainty of the data.

Thus, the novelty of this procedure is twofold: on the one hand, we use a minimal model taking into account the measurement error in the CGMS, and on the other hand, it is a tool that may help the physician to provide proper recommendations.

The proposed computational procedure is divided into two parts:

1. Calibration: using the model presented in [1], we solve the inverse problem of determining the model parameter values in such a way the output of the model captures the data (patient's glucose levels) and the possible measure errors of the electronic device (uncertainty).

2. Recommendation: considering the healthy glucose range $(70-180 \mathrm{mg} / \mathrm{dL})$ and all possible scenarios for administering insulin and eating carbohydrates, we perform model simulations for each scenario over four hours. Then, we analyse the results to select the best recommendation for the patient, i.e, the one with the higher time in range.

We apply this technique to two patients in three situations that cover most of the scenarios a diabetic patient may have, with high, medium and low glucose levels.

To the best of our knowledge, this is a new way of using minimal models in the prediction of the glucose levels of a patient.

The paper is organized as follows. In Section 10.2 we present the model describing the dynamics of the glucose in a patient. It is a discrete version of the one presented in [15] where the measure units have been changed to $\mathrm{mg} / \mathrm{dL}$. Section 10.3 deals with data and their uncertainty treatment to estimate the measurement errors. In Section 10.4 we show the model calibration, the design of scenarios about the administration of insulin and the consumption of carbohydrates, as well as the performance of the model simulations in each proposed scenario. Results and their 
analysis are presented in Sections 10.5 and 10.6, respectively. Finally, in Section 10.7 we present our conclusion.

\subsection{Model description}

This section introduces the mathematical model describing the dynamics of the glucose of diabetic patient. Starting from the Bergman minimal model (BMM) [13], in $[106,15]$ the authors extended the BMM through two modifications. First, they substituted the insulin action and insulin absorption models [94] by a second order insulin action model. Second, they added the second order linear carbohydrates sub-model from [67]. As a result, the minimal model (MM), which discretization is described in [1], can be written as the following system of five difference equations:

$$
\begin{aligned}
G_{t+1} & =G_{t}-X_{t} G_{t}-S_{g_{0}} G_{t}+U_{\text {endo }}+C \frac{U_{t}}{W}, \\
A_{t+1} & =A_{t}+V_{t} \\
V_{t+1} & =V_{t}-2 a_{g} V_{t}-a_{g}^{2} A_{t}+K_{g} a_{g}^{2} C h_{t}, \\
X_{t+1} & =X_{t}-a_{x} X_{t}+a_{x} X_{t}^{1} \\
X_{t+1}^{1} & =X_{t}^{1}-a_{x} X_{t}^{1}+K_{x} a_{x} \frac{I_{t}}{W}
\end{aligned}
$$

where

- $G_{t}$ is the glucose level of the patient at time $t$,

- $A_{t}$ is the gut glucose absorption at time $t$,

- $V_{t}$ is the variation rate of the gut glucose absorption at time $t$,

- $X_{t}$ is the insulin action at time $t$, and

- $X_{t}^{1}$ represents the intermediate insulin action at time $t$.

The model parameters are related with the daily clinics of the patient and his/her specific biology. The model parameters and constants are [1], 
- $C h_{t}$ are the level of ingested carbohydrates at time $t$,

- $I_{t}$ are the level of insulin at time $t$,

- $C=50 / 9,^{1}$

- $W$ is the weight of the patient,

- $S_{g_{0}}$ is the glucose effectiveness at zero insulin,

- $U_{\text {endo }}$ is the insulin independent endogenous glucose production,

- $a_{g}$ is the inverse of the meal time constant,

- $K_{g}$ is the unit-less bioavalibility of the meal of interest,

- $a_{x}$ is the inverse of the insulin absorption/action time constant, and

- $K_{x}$ is the insulin sensitivity.

In Table 10.1 we show the units of the model variables, parameters and data of the patient.

\begin{tabular}{|c|c||c|c||c|c|}
\hline \multicolumn{2}{|c}{ Variables } & \multicolumn{2}{c|}{ Data of the patient } & \multicolumn{2}{c|}{ Parameters } \\
\hline$A_{t}$ & $g / \mathrm{min}^{2}$ & $C h_{t}$ & $g / \mathrm{min}$ & $a_{g}$ & $1 / \mathrm{min}$ \\
$V_{t}$ & $g / \mathrm{min}^{2}$ & $I_{t}$ & $\mathrm{mU} / \mathrm{min}$ & $K_{g}$ & unit-less \\
$G_{t}$ & $\mathrm{mg} / d L$ & $C$ & $m g \times K g /(d L \times g)$ & $S_{g_{0}}$ & $1 / \mathrm{min}$ \\
$X_{t}$ & $1 / \mathrm{min}$ & $W$ & $K g$ & $U_{\text {endo }}$ & $\mathrm{mg} / \mathrm{dL}$ \\
$X_{t}^{1}$ & $1 / \mathrm{min}$ & & & $a_{x}$ & $1 / \mathrm{min}$ \\
& & & & $K_{x}$ & $\mathrm{~kg} / \mathrm{mU}$ \\
\hline
\end{tabular}

Table 10.1: Units of the model variables, data of the patient and parameters.

\footnotetext{
${ }^{1}$ In the original Minimal Model [106, 15] $C=50 / 9$, the constant which converts grams of glucose into mmol. Details can be seen in the Appendix of [1].
} 


\subsection{Data and their uncertainty}

Once we have introduced the model, we consider the levels of glucose of a specific patient given by $g_{t}$ for the time instants $t=1,2, \ldots n$. These glucose levels have been measured with an electronic device with, at least, $5 \%$ of measurement error described in the technical specifications. As we mentioned before, we want to obtain the set of parameter values that allow the model to capture the uncertainty due to errors in the measurement of the glucose. For this purpose, let us assume that each measured glucose level is represented by a random variable instead of a nominal value.

The errors in the measures taken by electronic devices are typically modelled using Gaussian Random Variables $N(\mu, \sigma)$ where $\mu$ is the mean and $\sigma$ the standard deviation [104]. In our case, assuming a measurement error of $5 \%$, for each time instant $t$ we take $\mathrm{g}_{t}$, the glucose level at time $t$, as the mean. Also, we take $0.05 \times \mathrm{g}_{t}$ as the standard deviation. Thus, $N\left(\mathrm{~g}_{t}, 0.05 \mathrm{~g}_{t}\right), t=1,2, \ldots n$ model the error measures of CGMS.

The $95 \%$ confidence interval (CI95\%) of $N\left(\mathrm{~g}_{t}, 0.05 \mathrm{~g}_{t}\right), t=1,2, \ldots n$ is given by percentiles 2.5 and 97.5 , that is, $L_{t}=0.902 \mathrm{~g}_{t}$ and $U_{t}=1.098 \mathrm{~g}_{t}$, respectively. Thus, the CI95\% of the data is $\left[L_{t}, U_{t}\right]=\left[0.902 \mathrm{~g}_{t}, 1.098 \mathrm{~g}_{t}\right], t=1,2, \ldots n$.

The CI95\% calculated above will allow us to look for the sets of model parameter values that best capture the data uncertainty.

\subsection{Procedure design}

As indicated above, our goal is to determine, knowing data of the levels of glucose of a patient in the last hour, when and how much insulin should be administered, and when and how much carbohydrates should be eaten to maximize the time in range of a patient. These crucial questions must be answered taking into account the uncertainty of the measured glucose levels to avoid possible critical situations for the patient.

Thus, let us assume that we have, measured via an electronic device, the levels of interstitial glucose of the patient in the last hour every five minutes, that is, 
$\mathrm{g}_{t_{i}}$, and their CI95\% $\left[L_{t_{i}}, U_{t_{i}}\right], t_{i}=5 \times i, i=1,2, \ldots 12$. We propose a procedure consisting of two parts:

- The calibration of the model of the equations (10.2)-(10.5) capturing the data and their uncertainty.

- The calculation of the amount of insulin and carbohydrates the patient should have and when, to preserve his/her glucose within healthy levels, as much as possible, over the next 4 hours.

\subsubsection{Model calibration capturing the data uncertainty}

The objective is to obtain a combination of different sets of parameters of the model (10.2)-(10.5) that best capture the data and their uncertainty. Without loss of generality we have predefined the amount of selected sets of parameters as $k$.

The model calibration is described in two steps.

- Step 1: Generation of a high number, $M$, of model outputs.

- Step 2: Selection of the $k$ sets of parameters from those $M$, in such a way the data uncertainty will be captured.

Step 1

The generation of the $M$ model outputs should be guided in such a way we can find model outputs close to the data. To do so, we use the optimization algorithm random PSO (rPSO) described in [80] to obtain good model outputs, and we store all the particles with their corresponding fitness. rPSO is a variation of the PSO algorithm version proposed by Khemka and Jacob where the velocities of the particles are considered pseudo random values. A particle $P_{j}$, is defined by a combination of the values of the set of parameters listed in Table 10.1:

$$
P_{j}=\left(a_{g_{j}}, K_{g_{j}}, S_{g_{0_{j}}}, U_{\mathrm{endo}_{j}}, a_{x_{j}}, K_{x_{j}}\right)
$$


denoting as $G_{t_{i}}^{P_{j}}$ the level of glucose returned by the model (model output) with the model parameters $P_{j}$, at time instant $t_{i}$. Then, we define the following fitness function $f$ as the sum of the errors of the 12 measures of glucose levels in the last hour, time instants $t_{i}=5 \times i, i=1, \ldots, 12$, that is

$$
f\left(P_{j}\right)=\sum_{i=1}^{12} \tau\left(G_{t_{i}}^{P_{j}}\right)
$$

where

$$
\tau\left(G_{t_{i}}^{P_{j}}\right)= \begin{cases}0, & \text { if } L_{t_{i}} \leq G_{t_{i}}^{P_{j}} \leq U_{t_{i}} \\ \min \left\{\left|G_{t_{i}}^{P_{j}}-L_{t_{i}}\right|,\left|G_{t_{i}}^{P_{j}}-U_{t_{i}}\right|\right\}, & \text { otherwise. }\end{cases}
$$

That is, the function $\tau$ returns zero if the model levels of glucose lie inside the data CI95\%, see Figure 10.1(a). Otherwise, $\tau$ returns the minimum distance of the model level of glucose, $G_{t_{i}}^{P_{j}}$ to the CI95\%, see Figure 10.1(b).

(a)

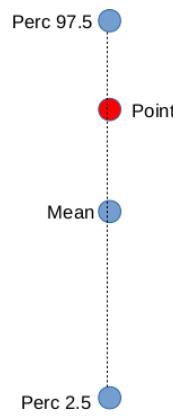

(b)

Figure 10.1: According to the expression (10.8), we have: (a) $\tau$ is zero if the level of glucose returned by the model, red dot, lie inside the CI95\%, blue dots. (b) $\tau$ is the minimum distance from the level of glucose returned by the model, red dot, and the CI95\%, blue dots, if they are not inside CI95\%. In this case, the distance between the red dot to the Perc 2.5 blue dot. 
We run rPSO 30 times with 700 model evaluations each run. All the evaluations performed during the 30 runs are stored, thus, we have a large enough number of evaluations to work with, $M=30 \times 700=21000$. For the shake of computational efficiency, we store the model parameter values $P_{j}$, the model output corresponding to $P_{j}$ in the time instants $t_{i}=5 \times i, i=1,2, \ldots 12$, and the fitness values $f\left(P_{j}\right)$.

Step 2

In order to select the appropriate $k$ model outputs that best capture the data uncertainty, we propose a specific algorithm. This algorithm is inspired in the regular Particle Swarm Optimization (PSO) algorithm where a proper fitness function is introduced. Let us denote $\Theta(j)$ the vector made up of the 12 model outputs obtained running the model with the parameters $P_{j}$, with $j=1, \ldots, M$ and $2 \leq k \leq M$, that is,

Index Parameters Output

$\begin{array}{ccc}1 & P_{1} & \Theta(1)=\left(G_{t_{1}}^{P_{1}}, G_{t_{2}}^{P_{1}}, \ldots, G_{t_{12}}^{P_{1}}\right), \\ 2 & P_{2} & \Theta(2)=\left(G_{t_{1}}^{P_{2}}, G_{t_{2}}^{P_{2}}, \ldots, G_{t_{12}}^{P_{2}}\right), \\ \vdots & \vdots & \vdots \\ M & P_{M} & \Theta(M)=\left(G_{t_{1}}^{P_{M}}, G_{t_{2}}^{P_{M}}, \ldots, G_{t_{12}}^{P_{M}}\right) .\end{array}$

Now, in the selection algorithm, a particle $E_{h}$ is

$$
E_{h}=\left\{I_{h_{1}}, \ldots, I_{h_{k}}\right\}
$$

where $E_{h}$ is a subset of $k$ indexes of $\{1, \ldots, M\}$. We evaluate each $E_{h}$ using the fitness function $F_{E} . F_{E}$ takes the model outputs corresponding to the indexes in $E_{h}$, calculates the mean and the CI95\% in each time instant and compares with the mean and the CI95\% of the data (see Figure 10.2). The function $F_{E}$ is defined following the next steps. 


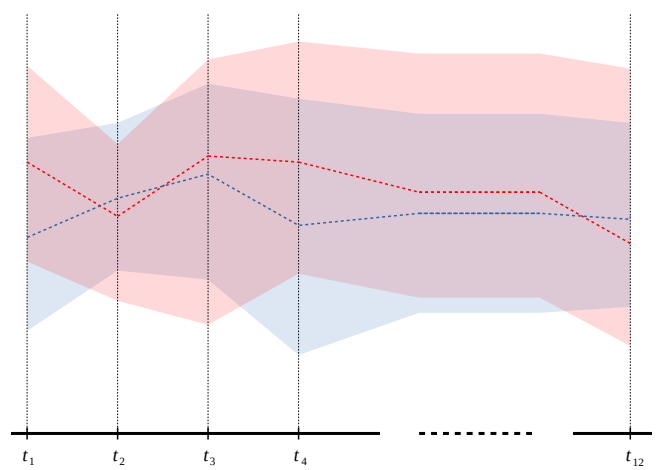

Figure 10.2: Fitness function $F_{E}$ measures the difference between the model output mean and CI95\% (red dashed line and red shadowed band) and the data mean and CI95\% (blue dashed line and blue shadowed band). The selection algorithm find sets of model parameter values in such a way that the red and the blue bands are as much similar as possible.

Step 1. Select the model output vectors $\Theta\left(I_{h_{1}}\right), \ldots, \Theta\left(I_{h_{k}}\right)$ and calculate the means $m_{h}=\left(m_{t_{1}}, \ldots, m_{t_{12}}\right)$, the percentiles $2.5, q_{h}=\left(q_{t_{1}}, \ldots, q_{t_{12}}\right)$, and the percentiles $97.5, Q_{h}=\left(Q_{t_{1}}, \ldots, Q_{t_{12}}\right)$.

Step 2. Calculate the error

$$
\begin{aligned}
F_{E}\left(E_{h}\right)= & \left\|m_{h}-\left(\mathrm{g}_{t_{1}}, \ldots, \mathrm{g}_{t_{12}}\right)\right\|_{2}+\left\|q_{h}-\left(L_{t_{1}}, \ldots, L_{t_{12}}\right)\right\|_{2}+ \\
& \left\|Q_{H}-\left(U_{t_{1}}, \ldots, U_{t_{12}}\right)\right\|_{2},
\end{aligned}
$$

where the 2-norm $\|\cdot\|_{2}$ of a vector is given by [116]

$$
\left\|\left(x_{1}, \ldots, x_{n}\right)\right\|_{2}=\sqrt{x_{1}^{2}+\cdots+x_{n}^{2}}
$$

and $\mathrm{g}_{t_{i}}, L_{t_{i}}, U_{t_{i}}$ for $i=1,2, \ldots 12$, are the data and the percentiles 2.5 and 97.5 , respectively, calculated in Section 10.3.

Note that the fitness function $F_{E}$ measures if a set of model outputs captures or not accurately the data uncertainty by comparing means and CI95\%. The selection algorithm search $k$ model outputs to minimize the $F_{E}$ function, and it is given by:

1. Initialization. 
- Initialize $H$ index subsets (particles), $E_{1}, \ldots, E_{H}$, with $k$ indexes from the set $\{1, \ldots, M\}$ chosen randomly without repetition (Eq. 10.10).

- Evaluate the fitness of all the particles, $F_{E}\left(E_{1}\right), \ldots, F_{E}\left(E_{P}\right)$.

- Define the individual subsets (particles) having the best fitnesses as $E_{i}^{\text {best }}=E_{i}, i=1, \ldots, H$ and the global subset (particle) with best fitness $E_{\text {global }}^{\text {best }}$ as the $E_{i}^{\text {best }}$ with minimum fitness.

2. For $i=1$ to $H$, extract randomly without repetition $k$ indexes from the union of the current particle $E_{i}$, its individual best $E_{i}^{\text {best }}$ and the global best $E_{\text {global }}^{\text {best }}$, and denote it as the new $E_{i}$.

3. Evaluate the fitness of all the new particles $F_{E}\left(E_{1}\right), \ldots, F\left(E_{H}\right)$.

4. Update the individual particle with best fitness $E_{i}^{\text {best }}, i=1, \ldots, H$ and the global best particle with fitness $E_{\text {global }}^{\text {best }}$.

5. Go to Step 2.

The $E_{\text {global }}^{\text {best }}$ returned in the last iteration of the algorithm will correspond to the $k$ indexes $\left\{j_{1}, \ldots, j_{k}\right\}$ which $95 \%$ confidence interval of the model output vectors $\Theta\left(j_{1}\right), \ldots, \Theta\left(j_{k}\right)$ capture the best the data uncertainty.

Here, there are some values that should be set to run the above algorithm, $k, H$ and $M$. In our case, $M$ can be up to 21000 , however, this figure is too high because the selection algorithm may take long time to find accurate solutions. Therefore, it is recommended to take much less outputs under the condition that these outputs "cover" the data uncertainty. To select the model outputs, we set a threshold so that about 3000 model outputs with error less than the threshold are chosen. This way, the performance of the algorithm is significantly improved.

On the other hand, the number of particles in the rPSO algorithm $H$ has to be also tuned. To perform the selection we used 30, 40, 50 and 60 particles. The best results have been obtained with $H=30$. In addition, we have to set $k$, i.e., the number of model outputs that are going to capture the data uncertainty. This number must be high enough to capture the data uncertainty accurately with a certain stability (that is, if we increase the value of $k$, the selected model outputs 
capture the data uncertainty with similar accuracy) and low enough to permit further complex calculations in affordable times. We performed tests with $k=100$ and $k=300$ obtaining similar results. Hence, we take $k=100$ and seek for the 100 model outputs that best capture the data uncertainty.

\subsubsection{Determining the best insulin-carbohydrates dosage to maintain healthy glucose levels}

Once $E_{\text {global }}^{\text {best }}$ have been found, we know the $k=100$ model outputs $\Theta\left(j_{1}\right), \ldots, \Theta\left(j_{k}\right)$, that best capture the data uncertainty. The model parameter values corresponding to these model outputs are $P_{j_{1}}, \ldots, P_{j_{k}}$, following the notation mentioned before.

Now, we set the healthy upper and lower bounds of glucose for diabetic patients, $\mathrm{U}=160 \mathrm{mg} / \mathrm{dL}$ and $\mathrm{L}=90 \mathrm{mg} / \mathrm{dL}$. Furthermore, we define $693(\mathrm{~m}, \mathrm{~s}, \mathrm{c})$ scenarios, corresponding to values of (minute, insulin dose, carbohydrates intake). That is

- over the next $m$ minutes, $m \in\{5,10,15\}$, administer

- $s m U$ of insulin, $s \in\{0,10000,20000,30000, \cdots, 190000,200000\}$ and, after 15 minutes, eat

- $c$ grams of carbohydrates, $c \in\{0,5,10,15,20,25,30,35,40,45,50\}$.

For a given scenario $(m, s, c)$, we take the $k$ model parameter values $P_{j_{i}}, i=1, \ldots k$, and run the model to obtain the model output (glucose levels) of the patient over the next 4 hours (240 minutes), considering that carbohydrates are taken 15 minutes after insulin doses. Thus, for each time instant, we have $k$ glucose values and we compute their percentiles 2.5 and 97.5 denoted as $\lambda=\left(\lambda_{1}, \ldots, \lambda_{240}\right)$ and $v=\left(v_{1}, \ldots, v_{240}\right)$, respectively. Similarly to expressions (10.7)-(10.8), we define the error function

$$
g(\lambda, v)=\sum_{i=1}^{240} \sigma\left(\lambda_{i}\right)+\sigma\left(v_{i}\right)
$$

where 


$$
\sigma(x)= \begin{cases}0, & \text { if } \mathrm{L}=90 \leq x \leq \mathrm{U}=160 \\ \min \{|\mathrm{L}-x|,|\mathrm{U}-x|\} & \text { otherwise }\end{cases}
$$

and, the error of the simulation is calculated. We repeat the procedure for all the 693 scenarios and among them, we select the scenario with minimum $g(\lambda, v)$. That is, the one which deviation from the healthy levels of glucose $\mathrm{U}$ and $\mathrm{L}$ is as small as possible, or equivalently, the time in range is maximum.

\subsection{Results}

The designed procedure, in the first step, attempts to explain the situation of the patient using a dynamic mathematical model. Then, in the second step we simulate scenarios, using the parameters that depict the current situation, in order to find which one is the best to be recommended to the patient.

To test the procedure, we are going to experiment with data from two real patients, namely Patient 1 and Patient 2, with high, medium and low levels of glucose. The weight of both patients is $W=70 \mathrm{~kg}$. In each of the 6 situations, we calibrate the model regarding the data uncertainty, we perform simulations considering the 693 scenarios, calculate the $95 \%$ confidence interval and select the one with the maximum time in range minimizing the error function (10.12)-(10.13).

This procedure has been been implemented in Python 3 software and executed in a computer with a Intel Core i7 7700HQ and $16 \mathrm{~Gb}$ of RAM. The calibration with uncertainty (Step 1, Section 10.4.1) takes an average of 160 seconds and Step 2 (Section 10.4.1) needs an average of 70 seconds to be executed. The selection of the best strategy explained in Section 10.4.2 takes an average of 155 seconds.

Graphical results can be seen in Figure 10.3, for Patient 1, and Figure 10.4 for Patient 2. In each graphic of both figures, we can see: on the left of the vertical grey dashed line, the result of the model calibration with the data and their uncertainty; on the right the prediction of the maximum time in range scenario for the patient. 
In both columns of the Figures 10.3 and 10.4, we represent the same. The difference is that on the left column we show all the trajectories and on the right only the 95\% confidence interval (band).

The bounds $\mathrm{U}=160 \mathrm{mg} / \mathrm{dL}$ and $\mathrm{L}=90 \mathrm{mg} / \mathrm{dL}$ are represented in the graphics by the dashed red horizontal lines.

In Table 10.2, we can see the best recommendation $(m, s, c)$ for each patient in each situation.

\begin{tabular}{|c|c|c|c|}
\hline $\begin{array}{c}\text { Patient } \\
\text { Scenario }\end{array}$ & $\begin{array}{c}\text { Minute } \\
\text { insulin }(\mathrm{m})\end{array}$ & $\begin{array}{c}\text { Quantity } \\
\text { insulin }(\mathrm{s})\end{array}$ & $\begin{array}{c}\text { Quantity } \\
\text { carbohydrates }(c)\end{array}$ \\
\hline Patient 1 / high level & 5 & $50000 \mathrm{mU}$ & $0 \mathrm{gr}$ \\
\hline Patient 1 / medium level & 15 & $0 \mathrm{mU}$ & $5 \mathrm{gr}$ \\
\hline Patient 1 / low level & 15 & $0 \mathrm{mU}$ & $10 \mathrm{gr}$ \\
\hline Patient 2 / high level & 5 & $80000 \mathrm{mU}$ & $0 \mathrm{gr}$ \\
\hline Patient 2 / medium level & 10 & $90000 \mathrm{mU}$ & $0 \mathrm{gr}$ \\
\hline Patient 2 / low level & 15 & $0 \mathrm{mU}$ & $15 \mathrm{gr}$ \\
\hline
\end{tabular}

Table 10.2: Minute when the insulin should be administered, which quantity and, after 15 minutes, the amount of carbohydrates the patient should eat for his/her best recommendation.

Furthermore, in Tables 10.3 and 10.4 we can see the obtained model parameter values for each patient in each situation. It is noteworthy that the values of $a_{x}=0.04$ and $a_{g}=0.03$ given in [15] for a linearisation of the minimal model (therapy parameter-based model), lie inside the obtained CI95\% and close to the obtained means in all the experiment situations. 


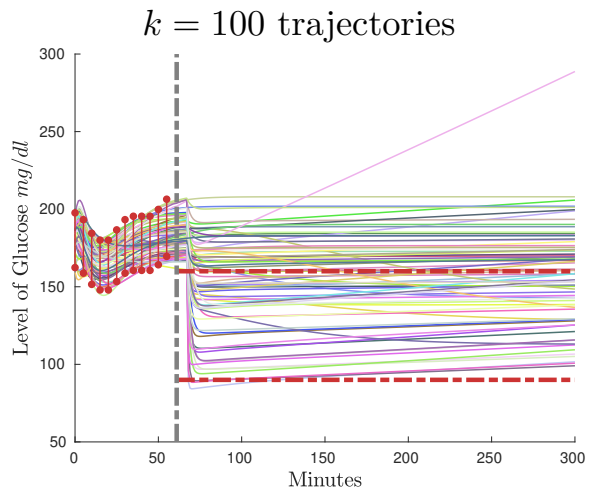

(a)

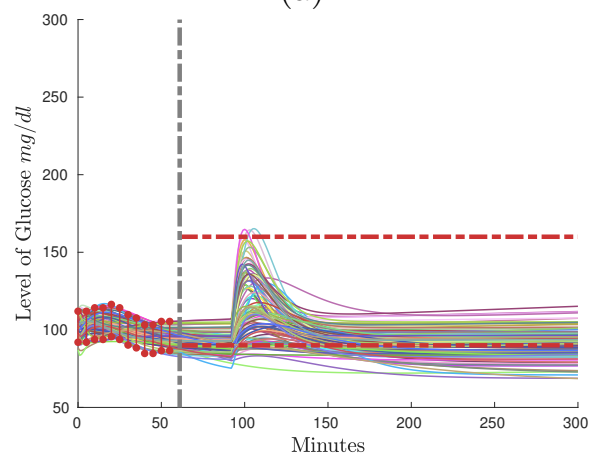

(c)

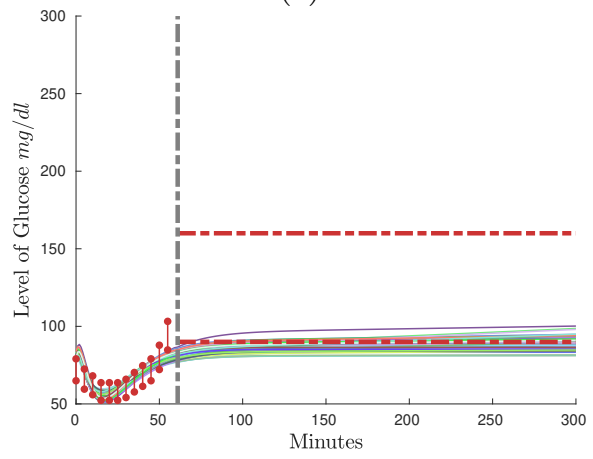

(e)

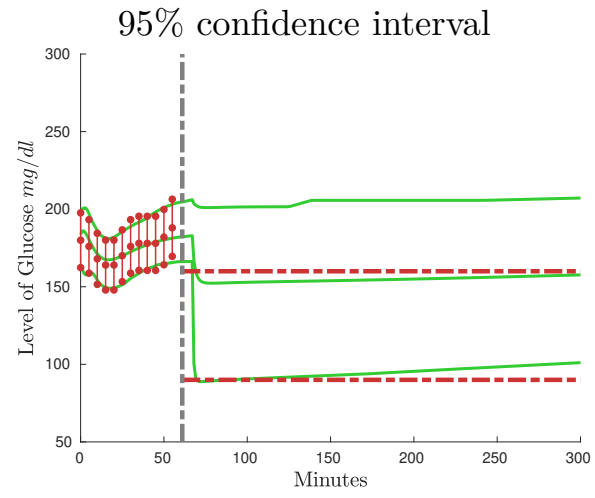

(b)

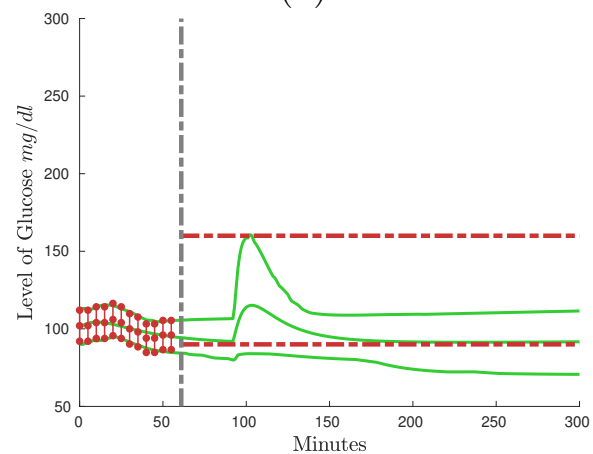

(d)

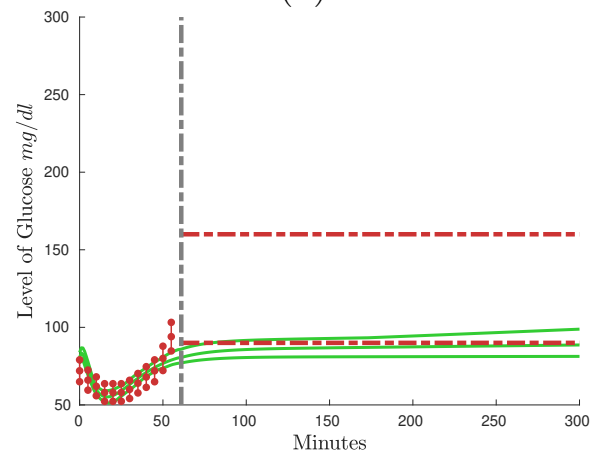

(f)

Figure 10.3: Patient 1. Three situations: (a) and (b) high levels of glucose; (c) and (d) medium levels of glucose; (e) and (f) low levels of glucose. On the left of the vertical grey line, the calibration with uncertainty. On the right of the grey vertical line, the prediction for the best scenario. The horizontal red dashed lines correspond to the healthy levels of glucose $\mathrm{U}=160$ and $\mathrm{L}=90$. On the left column, the $k=100$ selected model outputs in the calibration procedure and the $k=100$ simulations over the next 4 hours for the maximum time in range scenario. On the right column, the same but only the mean and the $95 \%$ confidence intervals. 


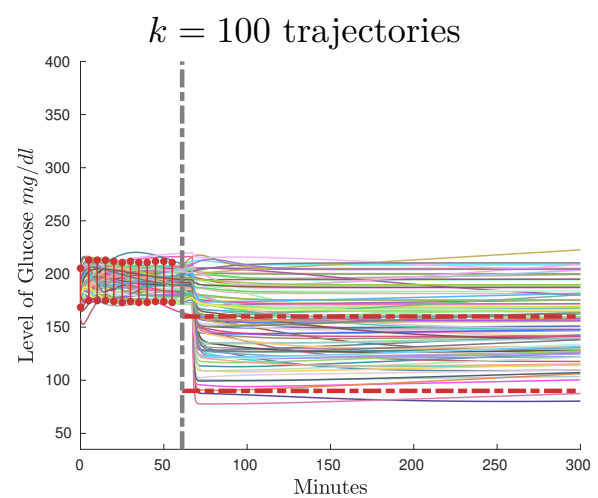

(a)

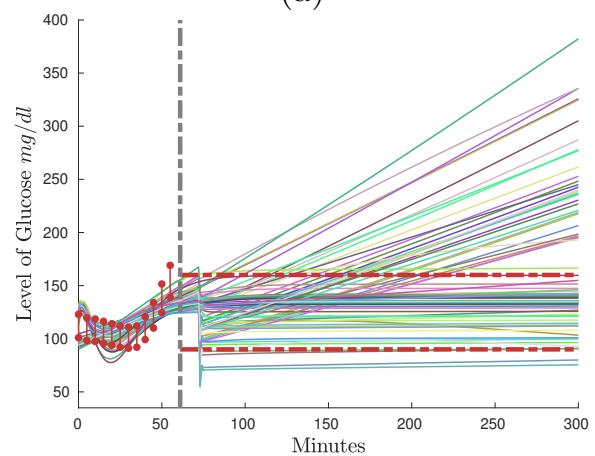

(c)

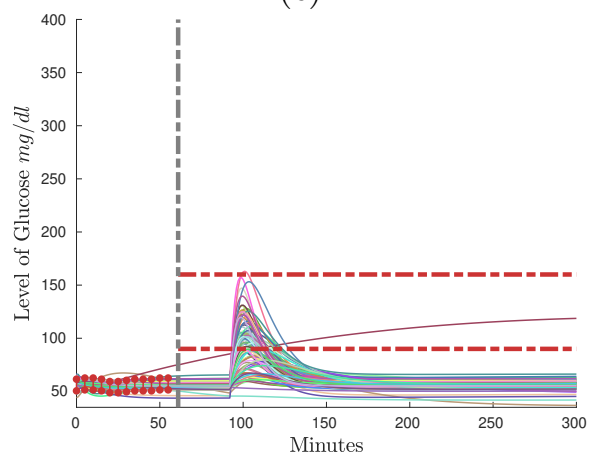

(e)

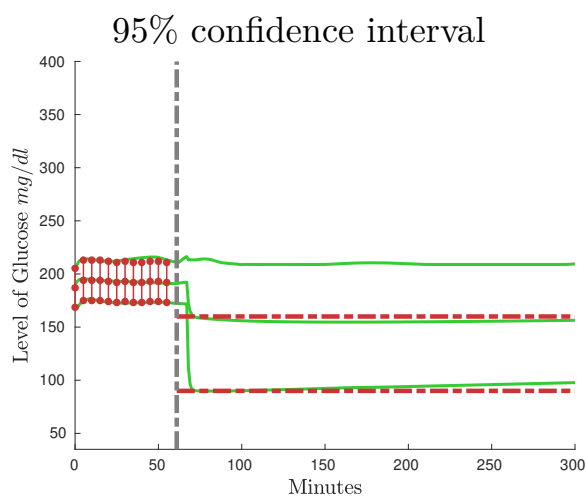

(b)

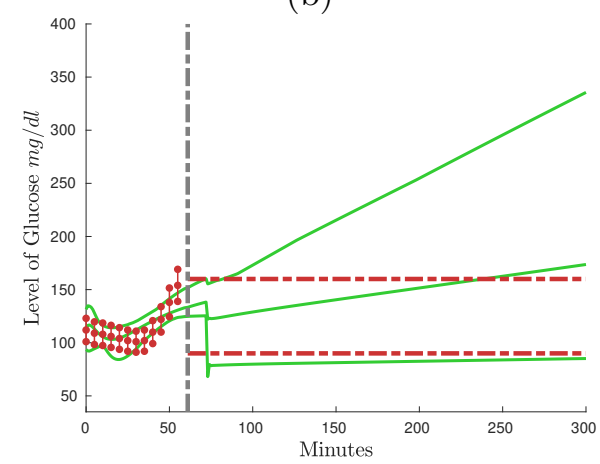

(d)

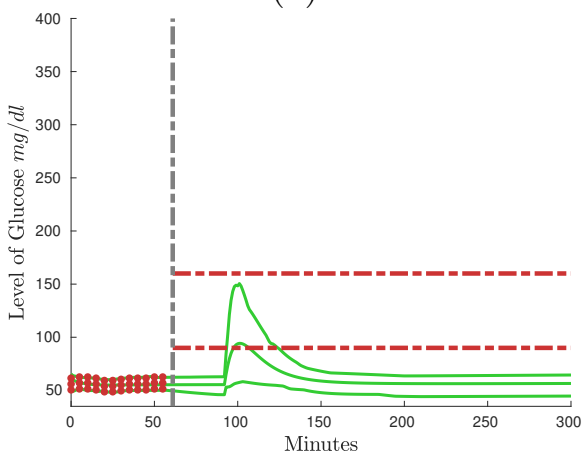

(f)

Figure 10.4: The same as Figure 10.3 but for Patient 2. 
Chapter 10. A random computational procedure to recommend insulin and carbohydrates intakes to diabetic patients

\begin{tabular}{|c|cccccc|}
\hline Patient 1-HL & $a_{g}$ & $K_{g}$ & $S_{g 0}$ & $U_{\text {endo }}$ & $a_{x}$ & $K_{x}$ \\
\hline Mean & 0.0872 & 570.4638 & 0.1904 & 64.479 & 0.0145 & 0.4217 \\
\hline 2.5 Percentile & 0.0142 & 0.0 & 0.0 & 32.7249 & 0.0 & 0.01 \\
\hline 97.5 Percentile & 0.1407 & 1421.9153 & 0.4525 & 87.0179 & 0.0286 & 0.9336 \\
\hline \hline Patient 1-LL & $a_{g}$ & $K_{g}$ & $S_{g 0}$ & $U_{\text {endo }}$ & $a_{x}$ & $K_{x}$ \\
\hline Mean & 0.074 & 274.6364 & 0.189 & 39.2978 & 0.0168 & 0.324 \\
\hline 2.5 Percentile & 0.0642 & 0.0 & 0.0086 & 26.0809 & 0.0 & 0.01 \\
\hline 97.5 Percentile & 0.0851 & 1016.648 & 0.4394 & 53.4756 & 0.0267 & 0.7757 \\
\hline \hline Patient 1-IL & $a_{g}$ & $K_{g}$ & $S_{g 0}$ & $U_{\text {endo }}$ & $a_{x}$ & $K_{x}$ \\
\hline Mean & 0.0731 & 682.3165 & 0.4499 & 60.8165 & 0.0153 & 0.3905 \\
\hline 2.5 Percentile & 0.0217 & 0.0 & 0.0882 & 25.344 & 0.0004 & 0.01 \\
\hline 97.5 Percentile & 0.1392 & 1337.3788 & 0.7627 & 82.8101 & 0.028 & 0.8771 \\
\hline
\end{tabular}

Table 10.3: Patient 1. Mean and CI95\% of the model parameter values in the situations HL (high level of glucose), LL (low level of glucose), IL (levels of glucose inside the healthy range).

\begin{tabular}{|c|cccccc|}
\hline Patient 2-HL & $a_{g}$ & $K_{g}$ & $S_{g 0}$ & $U_{\text {endo }}$ & $a_{x}$ & $K_{x}$ \\
\hline Mean & 0.0715 & 771.7842 & 0.1526 & 69.8948 & 0.0117 & 0.494 \\
\hline 2.5 Percentile & 0.0076 & 0.0 & 0.0 & 33.8751 & 0.0 & 0.01 \\
\hline 97.5 Percentile & 0.1351 & 1394.3786 & 0.4867 & 87.9031 & 0.0267 & 0.9074 \\
\hline \hline Patient 2-LL & $a_{g}$ & $K_{g}$ & $S_{g 0}$ & $U_{\text {endo }}$ & $a_{x}$ & $K_{x}$ \\
\hline Mean & 0.0915 & 816.4032 & 0.6083 & 49.5728 & 0.0152 & 0.4911 \\
\hline 2.5 Percentile & 0.0205 & 0.0 & 0.3322 & 33.4541 & 0.0 & 0.0168 \\
\hline 97.5 Percentile & 0.1414 & 1454.1741 & 0.762 & 64.1808 & 0.0285 & 0.9392 \\
\hline \hline Patient 2-IL & $a_{g}$ & $K_{g}$ & $S_{g 0}$ & $U_{\text {endo }}$ & $a_{x}$ & $K_{x}$ \\
\hline Mean & 0.0421 & 692.7575 & 0.2968 & 56.2248 & 0.0111 & 0.4074 \\
\hline 2.5 Percentile & 0.0 & 0.0 & 0.0 & 28.5421 & 0.0 & 0.01 \\
\hline 97.5 Percentile & 0.0994 & 1450.6895 & 0.6757 & 86.5477 & 0.0284 & 0.9334 \\
\hline
\end{tabular}

Table 10.4: Patient 2. Mean and CI95\% of the model parameter values in the situations HL (high level of glucose), LL (low level of glucose), IL (levels of glucose inside the healthy range). 


\subsection{Analysis}

The calibrations are fairly well except, maybe, in the low case of the Patient 1 (Figure 10.3(e) and (f)) where the data uncertainty in the left part is not properly captured. However, most of predictions should be considered as reliable. Nevertheless, the $95 \%$ confidence intervals are not completely confined in the bounds $\mathrm{U}=160 \mathrm{mg} / \mathrm{dL}$ and $\mathrm{L}=90 \mathrm{mg} / \mathrm{dL}$ and there may be potential serious issues.

To estimate the probability to reach these potential concerning issues, for each time instant we take the values of the $k=100$ trajectories and determine the probability to be above $180 \mathrm{mg} / \mathrm{dL}$, to be between $70 \mathrm{mg} / \mathrm{dL}$ and $180 \mathrm{mg} / \mathrm{dL}$, and to be below $70 \mathrm{mg} / \mathrm{dL}$. These new upper and lower bounds are less conservatives than the old ones and their differences may be considered as an usual safety band in order to provide extra patient's protection.

In Figure 10.5 we can see the probability to be inside the band $70 \mathrm{mg} / \mathrm{dL}-$ $180 \mathrm{mg} / \mathrm{dL}$ or above or below, in each minute over the following 4 hours, in all the 6 studied experiments. We expect this figure jointly with the information in Table 10.2 may be helpful to give accurate recommendations to the diabetic patients. For instance, for the Patient 2 Figure 10.5(d), it would be interesting a reevaluation after 3 hours and a half because the probability of being in safe levels of glucose is decreasing. Or in Figure 10.5(f), the patient should be reevaluated very soon in order to try to keep his/her levels of glucose within appropriate ranges. On the other hand, the predictions for the Patient 1 are fairly stable and safe over the time in the three scenarios. 
Chapter 10. A random computational procedure to recommend insulin and carbohydrates intakes to diabetic patients

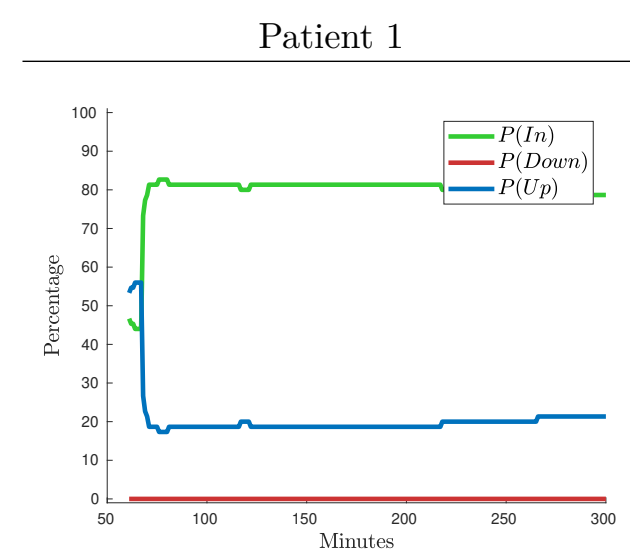

(a)

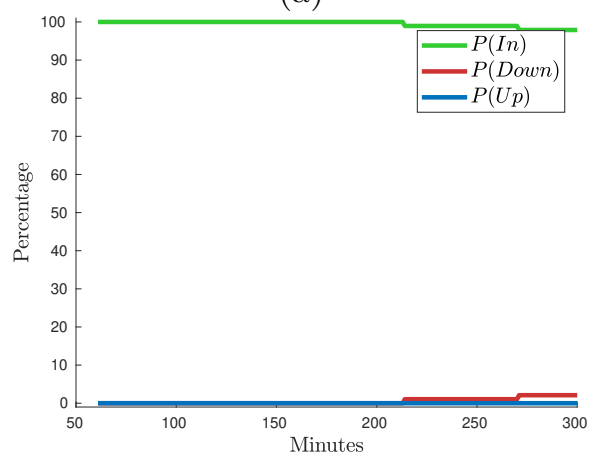

(c)

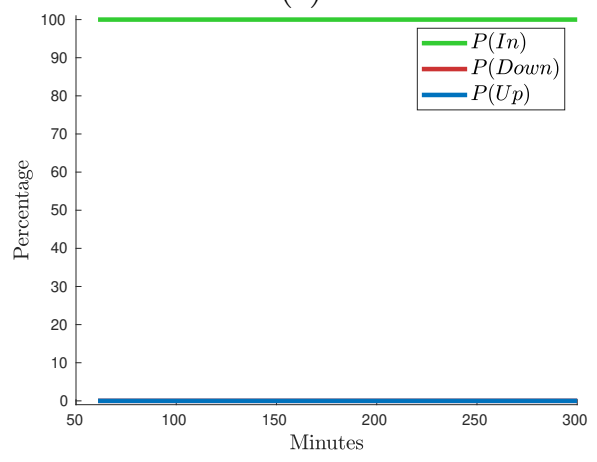

(e)
Patient 2

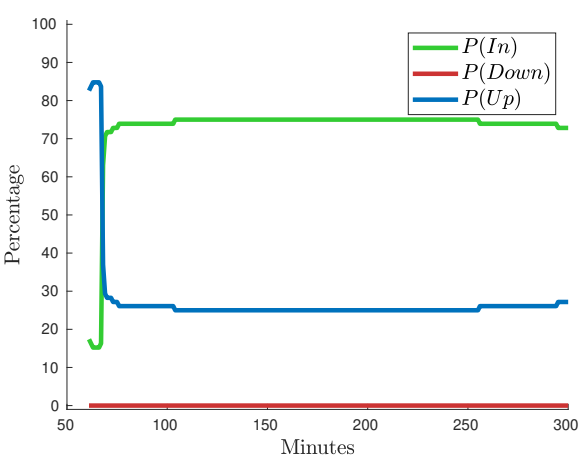

(b)

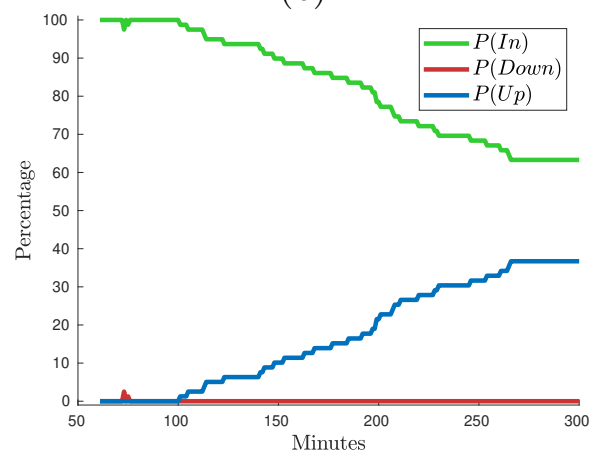

(d)

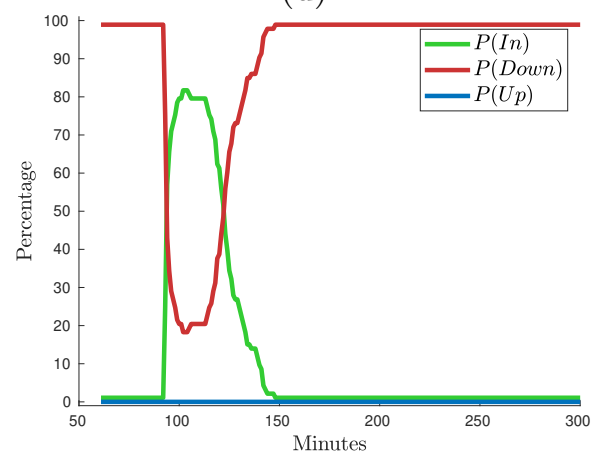

(f)

Figure 10.5: Probability to remain within the healthy levels of glucose $[70 \mathrm{mg} / \mathrm{dL}$, $180 \mathrm{mg} / \mathrm{dL}$ ] over the time, in the 6 studied cases. Green lines show the probability to be inside the healthy levels of glucose [70,180]; blue lines stand for the probability to reach levels of glucose greater than 180; red lines show the probability to reach lower levels of glucose than 70 . 


\subsection{Conclusion}

In this paper we propose a computational procedure to help the diabetic patients and physicians to explain the current levels of glucose and make the appropriate decisions about the doses of insulin to be administered and carbohydrates to be eaten in the proper time instants to maximize the time in range over the next four hours. The major interest of this procedure lies on the fact that to the best of our knowledge, it is only that provide recommendations taking into account the data uncertainty.

The proposed procedure, in the first step, determines the model parameter values that allow the model to describe the levels of glucose and the measure errors (uncertainty) in the last hour.

With the obtained model parameters values and the model, we simulate possible scenarios of insulin administration and carbohydrates consumption in order to find which one is the best to be recommended to the patient.

This procedure has been used in two real patients in three different situations: when the patient has high, medium and low levels of glucose currently.

Experimental results for the two patients are representative of the most common situations that a patient can face, according to physician indications. The results seem to be very promising and allow us to provide, for every patient in every situation, the probability to overpass the safe levels of glucose, moving to concerning situations.

The presented computational procedure can be adapted easily to particular situations of the patient only having his/her levels of glucose in the last hour. For instance, if it would be preferable to eat twice over the next 4 hours, or the patient is going to do some exercise. This would require to design new situations to simulate. Also, the procedure may re-evaluate the patient's situation regularly, updating the predictions and the actions to be done. 
This procedure could be deployed in the cloud in such a way that the patients, with an app installed in his/her smartphone, could get the best recommendations in order to keep the glucose in the safe levels as much time as possible.

\section{Chapter contribution}

With regard to this chapter, the $\mathrm{PhD}$ candidate has contributed by working in its complete development with more emphasis on the development and implementation of the random technique to recommend insulin and carbohydrates intakes to a diabetic patient. 


\section{Chapter

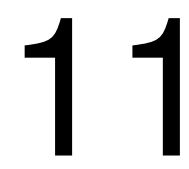

General Conclusions

Applying the techniques described along this dissertation, in models formulated by classical or fractional difference or differential equations, more exhaustive descriptions of a real phenomena can be achieved. We not only obtain a deterministic description of the process, but also we can describe the intrinsic randomness of the phenomena.

In this dissertation uncertainty quantification techniques have been developed in two mathematical areas. The similarity of these two fields are the applicability in the modelling processes. On the one hand, it is well known the use of fractional differential equations in the modelling of real phenomena. In this dissertation, fractional differential equations are studied considering uncertainties in their formulation. On the other hand, computational techniques to quantify the uncertainty support us in the modelling processes. Here, computational methods to study the randomness have been developed and validate in biological dynamic problems.

In Chapters 2, 3, 4, 5 and 6 fractional calculus have been extended in a random framework, concretely the in mean square sense. Initial value problems have been proposed. They have been solved applying a generalized version of the Frobenius 
method obtaining a random generalized power series solution. Mild conditions to guarantee the convergence in mean square sense have been established. Expressions for the main statistical moments and for the first probability density function have been also developed.

Nevertheless, despite the above mentioned chapters the exact solution has been obtained, it is not always possible. In this dissertation one chapter has been devoted (Chapter 7) to develop a numerical method in order to approximate the solution stochastic process from a random fractional initial value problem.

Throughout this thesis, the utility of fractional calculus in mathematical modelling has been pointed out frequently. Chapter 8 has been devoted to illustrate the capability of this mathematical tool to model the outbreaks of meningococcus W-135 in Spain. To do it, a computational method to quantify the uncertainty of meningococcus carriers among the Spanish population has been developed and applied to a fractional Lotka-Volterra model. A confidence interval that describes the percentage of carriers of meningococcus $\mathrm{W}$ over the next few years has been obtained.

Finally, the last two Chapters (Chapter 9 and 10) have been devoted to develop computational strategies to quantify the uncertainty in mathematical models. In particular, Chapter 9 has been devoted to establish a computational approach to obtain the first probability density function of the randomized logistic model that describes the growth of breast tumour. The objective of Chapter 10 has been to determine a recommendation of insulin shoots and carbohydrates intakes for a diabetic patient in order to maintain their glucose level in healthy ranges taking into account the uncertainty given by real data. 


\section{Bibliography}

[1] L. Acedo, M. Botella, J. C. Cortés, J. I. Hidalgo, E. Maqueda, and R. J. Villanueva. "Swarm hybrid optimization for a piecewise model fitting applied to a glucose model". In: Journal of Systems and Information Technology 20.4 (2018), pp. 404-416. DOI: $10.1108 /$ jsit-10-2017-0103 (cit. on pp. 207 sqq.).

[2] L. Acedo, C. Burgos, J. C. Cortés, and R. J. Villanueva. "Probabilistic prediction of outbreaks of meningococcus W-135 infections over the next few years in Spain". In: Physica A: Statistical Mechanics and its Applications 486 (2017), pp. 106-117. DOI: $10.1016 /$ j . physa. 2017.05 .043 (cit. on p. 183).

[3] A. Aghili and H. Zeinali. "Solution to fractional Schrodinger and Airy differential equations via integral transforms". In: British Journal of Mathematics \& Computer Sciences 4.18 (2014), pp. 2630-2664. DOI: 10.9734/ BJMCS/2014/11064 (cit. on p. 108).

[4] J. F. Aguilera, A. Perrocheau, C. Meffre, S. Hahné, W135 Working Group, et al. "Outbreak of serogroup W135 meningococcal disease after the Hajj pilgrimage, Europe, 2000". In: Emerging infectious diseases 8.8 (2002), p. 761. DOI: 10.3201/eid0808.010422 (cit. on pp. 163 sq.).

[5] G. Al-Gaphari, N. Alsohybe, et al. "Survey: mathematical models for the regulation mechanism of glucose pancrea's hormones in the human body". In: International Journal of Advanced Research in Computer Science 11.2 (2020), p. 5. DOI: 10.26483/ijarcs.v11i2.6512 (cit. on p. 207).

[6] A. Ansari. "The fractional Airy transform". In: Hacettepe Journal of Mathematics and Statistics 44.4 (2015), pp. 761-766. DOI: 10.15672/HJMS . 2015449440 (cit. on p. 108). 
[7] L. Arnold. Stochastic Differential Equations: Theory and Applications. New York: Krieger Publishing Company, 1992. ISBN: 978-981-277-063-9 (cit. on p. 9).

[8] American Diabetes Association. "Diagnosis and Classification of Diabetes Mellitus". In: Diabetes Care 32.Supplement_1 (Dec. 2008), S62-S67. DoI: 10.2337/dc09-s062 (cit. on p. 206).

[9] T. Atanacković, M. Nedeljkov, S. Pilipović, and D. Rajter-Ćiri. "Dynamics of a fractional derivative type of a viscoelastic rod with random excitation". In: Fractional Calculus and Applied Analysis 18.5 (2015), 1232-1251. DOI: 10.1515/fca-2015-0071 (cit. on p. 4).

[10] F. Atici and P. Eloe. "Initial value problems in discrete fractional calculus". In: Proceedings of the American Mathematical Society 137.3 (2009), pp. 981989. DOI: 10.1090/S0002-9939-08-09626-3 (cit. on p. 169).

[11] N. C. Atuegwu, L. R. Arlinghaus, X. Li, A. B. Chakravarthy, V. G. Abramson, M. E. Sanders, and T. E. Yankeelov. "Parameterizing the logistic model of tumor growth by DW-MRI and DCE-MRI data to predict treatment response and changes in breast cancer cellularity during neoadjuvant chemotherapy". In: Translational oncology 6.3 (2013), pp. 256-264. DOI: 10.1593/tlo.13130 (cit. on pp. 187 sq.).

[12] N. Bellomo and E. de Angelis. Selected Topics in Cancer Modeling: Genesis, Evolution, Immune Competition, and Therapy. Springer Science \& Business Media, 2008. ISBN: 978-0-8176-4712-4 (cit. on pp. 186 sq.).

[13] R. N. Bergman, Y. Z. Ider, C. R. Bowden, and C. Cobelli. "Quantitative estimation of insulin sensitivity." In: American Journal of PhysiologyEndocrinology and Metabolism 236.6 (June 1979), E667. DOI: 10.1152/ ajpendo.1979.236.6.e667 (cit. on p. 209). 
[14] Z. T. Bloomgarden. "Consequences of Diabetes: Cardiovascular disease". In: Diabetes Care 27.7 (June 2004), pp. 1825-1831. DOI: 10.2337/diacare. 27.7.1825 (cit. on p. 206).

[15] A. Bock, G. François, and D. Gillet. "A therapy parameter-based model for predicting blood glucose concentrations in patients with type 1 diabetes". In: Computer Methods and Programs in Biomedicine 118.2 (2015), pp. 107123. DOI: $10.1016 /$ j.cmpb. 2014.12.002 (cit. on pp. 207 sqq., 219).

[16] D. A Botesteanu, S. Lipkowitz, J. M. Lee, and D. Levy. "Mathematical models of breast and ovarian cancers". In: Wiley Interdisciplinary Reviews: Systems Biology and Medicine 8.4 (2016), pp. 337-362. DOI: 10.1002/wsbm . 1343. (cit. on p. 186).

[17] N. Boyd, L. Martin, A. Gunasekara, O. Melnichouk, G. Maudsley, C. Peressotti, M. Yaffe, and S. Minkin. "Mammographic density and breast cancer risk: evaluation of a novel method of measuring breast tissue volumes". In: Cancer Epidemiology and Prevention Biomarkers 18.6 (2009), pp. 17541762. DOI: 10.1158/1055-9965.EPI-09-0107 (cit. on p. 186).

[18] F. Brauer and C. Castillo-Chavez. Mathematical Models in Population Biology and Epidemiology. Vol. 2. Springer, 2012. ISBN: 978-1-4614-1685-2 (cit. on p. 188).

[19] A. Brown. Time-in-Range: What's an Achievable Goal with Diabetes? https://diatribe.org/time-range-whats-achievable-goal-diabetes. 2017 (cit. on p. 206).

[20] C. Burgos, J. Calatayud, J. C. Cortés, and A. Navarro-Quiles. "A full probabilistic solution of the random linear fractional differential equation via the random variable transformation technique". In: Mathematical Methods in the Applied Sciences 41.18 (2018), pp. 9037-9047. DOI: 10.1002/mma. 4881 (cit. on p. 91). 
[21] C. Burgos, J. Calatayud, J. C. Cortés, and L. Villafuerte. "Solving a class of random non-autonomous linear fractional differential equations by means of a generalized mean square convergent power series". In: Applied Mathematics Letters 78 (2018), pp. 95-104. DOI: 10.1016/j.aml.2017.11.009 (cit. on pp. 82, 106).

[22] C. Burgos, J. C. Cortés, A. Debbouche, L. Villafuerte, and R. J. Villanueva. "Random fractional generalized Airy differential equations: A probabilistic analysis using mean square calculus". In: Applied Mathematics and Computation 352 (2019), pp. 15-29. DOI: 10.1016/j.amc.2019.01.039 (cit. on p. 135).

[23] C. Burgos, J. C. Cortés, D. Martínez-Rodríguez, and R. J. Villanueva. "Computational modelling with uncertainty of frequent users of e-commerce in Spain using an age-group dynamic nonlinear model with varying size population". In: Advances in Complex Systems 22.4 (2019), pp. 1950009-1. DOI: 10.1142/S0219525919500097 (cit. on p. 1).

[24] C. Burgos, J. C. Cortés, L. Villafuerte, and R. J. Villanueva. "Extending the deterministic Riemann-Liouville and Caputo operators to the random framework: A mean square approach with applications to solve random fractional differential equations". In: Chaos, Solitons \& Fractals 102 (2017), pp. 305-318. DOI: 10.1016/j.chaos.2017.02.008 (cit. on pp. 44, 60, 82).

[25] C. Burgos, J. C. Cortés, L. Villafuerte, and R. J Villanueva. "Mean square calculus and random linear fractional differential equations: Theory and applications". In: Applied Mathematics and Nonlinear Sciences 2.2 (2017), pp. 317-328. DOI: 10.21042/amns.2017.2.00001 (cit. on p. 156).

[26] C. Burgos, J. C. Cortés, L. Villafuerte, and R. J. Villanueva. "Mean square convergent numerical solutions of random fractional differential equations: Approximations of moments and density". In: Journal of Computational and Applied Mathematics (2020), p. 112925. DOI: 10.1016/j.cam.2020.112925 (cit. on p. 160). 
[27] C. Burgos, J. C. Cortés, L. Villafuerte, and R. J. Villanueva. "Solving random mean square fractional linear differential equations by generalized power series: Analysis and computing". In: Journal of Computational and Applied Mathematics 339 (2018), pp. 94-110. DOI: 10.1016/j.cam.2017.12.042 (cit. on p. 74).

[28] C. Burgos, D. Cortés J. C. Martínez-Rodríguez, and R. J. Villanueva. "Modeling breast tumor growth by a randomized logistic model: A computational approach to treat uncertainties via probability densities". In: The European Physical journal Plus 135.826 (2020). DOI: 10.1140/epjp/s13360-02000853-3 (cit. on p. 203).

[29] C. Burgos, N. García-Medina, D. Martínez-Rodríguez, and R. J. Villanueva. "Mathematical modeling of the dynamics of the bladder cancer and the immune response applied to a patient: Evolution and short-term prediction". In: Mathematical Methods in the Applied Sciences 42.17 (2019), pp. 57465757. DOI: 10.1002/mma. 5536 (cit. on p. 186).

[30] G. Q. Cai and W. Q. Zhu. Elements of Stochastic Dynamics. Singapure: World Scientific, 2016. ISBN: 987.981-4723-32-9 (cit. on p. 25).

[31] G. Calbo, J. C. Cortés, L. Jódar, and L. Villafuerte. "Solving the random Legendre differential equation: Mean square power series solution and its statistical functions". In: Computers and Mathematics with Applications 61.9 (2011), pp. 2782-2792. DOI: 10.1016/j.camwa.2011.03.045 (cit. on p. 25).

[32] K. Cartwright et al. Meningococcal disease. Wiley Chichester, 1995. IsBn: 978-0471952596 (cit. on p. 162).

[33] M. C. Casabán, J. C. Cortés, A. Navarro-Quiles, J. V. Romero, M. D. Roselló, and R. J. Villanueva. "A comprehensive probabilistic solution of random SIS-type epidemiological models using the random variable transformation technique". In: Communications in Nonlinear Science and 
Numerical Simulation 32 (2016), pp. 199-210. DOI: $10.1016 /$ j.cnsns . 2015.08.009 (cit. on pp. 76, 188).

[34] M. C. Casabán, J. C. Cortés, J. V. Romero, and M. D. Roselló. "Probabilistic solution of random SI-type epidemiological models using the Random Variable Transformation technique". In: Communications in Nonlinear Science and Numerical Simulation 24 (1-3 2015), pp. 86-97. DOI: 10.1016/ j.cnsns.2014.12.016 (cit. on p. 188).

[35] M. C. Casabán, J. C. Cortés, J. V. Romero, and M.D. Roselló. "Solving random homogeneous linear second-order differential equations: a full probabilistic description". In: Mediterranean Journal of Mathematics 13.6 (2016), pp. 3817-3836. DOI: 10.1007/s00009-016-0716-6 (cit. on p. 76).

[36] G. Casella and R.L. Berger. Statistical Inference. Duxbury Advanced Series. New York: Brooks Cole, 2002. ISBN: 9780534243128 (cit. on p. 119).

[37] H. Caysa, S. Hoffmann, J. Luetzkendorf, L. P. Mueller, S. Unverzagt, K. Mäder, and T. Mueller. "Monitoring of xenograft tumor growth and response to chemotherapy by non-invasive in vivo multispectral fluorescence imaging". In: PLoS One 7.10 (2012), e47927. DOI: 10.1371/journal.pone. 0047927 (cit. on p. 191).

[38] S. Chakraborty, A. Debbouche, and P. K. Roy. "A mathematical modelling for treatment of HPV associated cervical cancer: NK and effector T cell based control study". In: Nonlinear Studies 27.2 (2020), pp. 325-336 (cit. on p. 186).

[39] H. Christensen, M. Hickman, W. J. Edmunds, and C. L. Trotter. "Introducing vaccination against serogroup B meningococcal disease: an economic and mathematical modelling study of potential impact". In: Vaccine 31.23 (2013), pp. 2638-2646. DOI: 10.1016/j.vaccine.2013.03.034 (cit. on p. 164). 
[40] M. P. Christiansen et al. "A Prospective multicenter evaluation of the accuracy of a novel implanted continuous glucose sensor: PRECISE II". In: Diabetes Technology \&5 Therapeutics 20.3 (Mar. 2018), pp. 197-206. DOI: 10.1089/dia.2017.0142 (cit. on p. 206).

[41] M. Clerc. Particle Swarm Optimization. New York: Wiley-ISTE, 2013. IsBn: 978-1-905-20904-0 (cit. on p. 104).

[42] J. C. Cortés, L. Jódar, J. Camacho, and L. Villafuerte. "Random Airy type differential equations: mean square exact and numerical solutions". In: Computers and Mathematics with Applications 60.5 (2010), pp. 1237-1244. DOI: $10.1016 /$ j.camwa. 2010.05.046 (cit. on pp. 25, 44, 108, 122 sq.).

[43] J. C. Cortés, A. Navarro-Quiles, J. V. Romero, and M. D. Roselló. "(CMMSE2018 paper) Solving the random Pielou logistic equation with the random variable transformation technique: theory and applications". In: Mathematical Methods in the Applied Sciences 42.17 (2019), pp. 5708-5717. DOI: 10.1002/mma. 5440 (cit. on pp. 189 sq.).

[44] J. C. Cortés, F. J. Santonja, A. C. Tarazona, R. J. Villanueva, and J. Villanueva-Oller. "A probabilistic estimation and prediction technique for dynamic continuous social science models: the evolution of the attitude of the Basque Country population towards ETA as a case study". In: Applied Mathematics and Computation 264 (2015), pp. 13-20. DOI: 10.1016/j . amc.2015.03.128 (cit. on pp. 1, 164, 171).

[45] J. C. Cortés, P. Sevilla-Peris, and L. Jódar. "Analytic-numerical approximating processes of diffusion equation with data uncertainty". In: Computers and Mathematics with Applications 49.7-8 (2005), pp. 1255-1266. DOI: 10.1016/j. camwa.2004.05.015 (cit. on p. 14).

[46] J. C. Cortés, L. Villafuerte, and C. Burgos. "A mean square chain rule and its applications in solving the random Chebyschev differential equation". In: Mediterranean Journal of Mathematics 14.1 (2017), pp. 1-14. DOI: 10.1007/s00009-017-0853-6 (cit. on pp. 13, 55 sq.). 
[47] A. Cunha Jr, R. Nasser, R. Sampaio, H. Lopes, and K. Breitman. "Uncertainty quantification through the Monte Carlo method in a cloud computing setting". In: Computer Physics Communications 185.5 (2014), pp. 13551363. DOI: $10.1016 /$ j.cpc. 2014.01 .006 (cit. on p. 1).

[48] P. De Wals. "Immunization strategies for the control of serogroup C meningococcal disease in developed countries". In: Expert Review of Vaccines 5.2 (2006), pp. 269-275. DOI: 10.1586/14760584.5.2.269 (cit. on p. 162).

[49] A. Debbouche and J. J. Nieto. "Sobolev type fractional abstract evolution equations with nonlocal conditions and optimal multi-controls". In: Applied Mathematics and Computation 245 (2014), pp. 74-85. DOI: $10.1016 / \mathrm{j}$. amc.2014.07.073 (cit. on pp. 2, 4).

[50] D. Delen, G. Walker, and A." Kadam. "Predicting breast cancer survivability: a comparison of three data mining methods". In: Artificial Intelligence in Medicine 34.2 (2005), pp. 113 -127. ISSN: 0933-3657. DOI: $10.1016 / \mathrm{j}$. artmed.2004.07.002 (cit. on p. 186).

[51] L. Devroye. "Nonuniform random variate generation". In: Handbooks in operations research and management science 13 (2006), pp. 83-121. DOI: 10.1016/S0927-0507(06)13004-2 (cit. on p. 195).

[52] K. Diethelm. The Analysis of Fractional Differential Equations: An ApplicationOriented Exposition Using Differential Operators of Caputo Type. Springer, 2010. ISBN: 978-3-642-14573-5 (cit. on p. 110).

[53] F. A. Dorini, M. S. Cecconello, and L.B. Dorini. "On the logistic equation subject to uncertainties in the environmental carrying capacity and initial population density". In: Communications in Nonlinear Science and Numerical Simulation 33 (2016), pp. 160-173. DOI: 10.1016/j.cnsns.2015. 09.009 (cit. on pp. 2, 76).

[54] H. Enderling, M. A. J. Chaplain, A. R. A. Anderson, and J. S Vaidya. "A mathematical model of breast cancer development, local treatment and 
recurrence". In: Journal of theoretical biology 246.2 (2007), pp. 245-259. DOI: $10.1016 / j \cdot j$ tbi.2006.12.010 (cit. on p. 187).

[55] M. Ezechiáš and T. Cajthaml. "Receptor partial agonism and method to express receptor partial activation with respect to novel full logistic model of mixture toxicology". In: Toxicology 393 (2018), pp. 26-33. DOI: 10.1016/j.tox.2017.10.012 (cit. on p. 188).

[56] V. A. Fonseca et al. "Continuous Glucose Monitoring: a consensus conference of the American Associatin of Clicinal Endocrinologists and American College of Endocrinology". In: Endocrine Practice 22.8 (Aug. 2016), pp. 1008-1021. DOI: 10.4158/ep161392.cs (cit. on p. 206).

[57] C. Fulai, L. Xiannan, and Z. Yong. "Existence results for nonlinear fractional difference equation". In: Journal of Advances in Difference Equations 2011.1 (2011), pp. 1-12. DOI: 10.1155/2011/713201 (cit. on p. 170).

[58] A. K. Golmankhaneh, R. Arefi, and D. Baleanu. "Synchronization in a nonidentical fractional order of a proposed modified system". In: Journal of Vibration and Control 21 (6 2015), pp. 1154-1161. DOI: 10.1177 / 1077546313494953 (cit. on p. 3).

[59] A. K. Golmankhaneh, N. A. Porghoveh, and D. Baleanu. "Mean square solutions of second-order random differential equations by using homotopy analysis method". In: Romanian Reports in Physics 65 (2013), pp. 350-362 (cit. on p. 3).

[60] R. Gorenflo, A. A. Kilbas, F. Mainardi, and Sergei V. R. Mittag-Leffler Functions, Related Topics and Applications. Berlin Heidelberg: SpringerVerlag, 2014. ISBN: 978-3-662-52324-7 (cit. on pp. 15, 41).

[61] E. F D. Goufo, Y. Khan, and Q. A. Chaudhry. "HIV and shifting epicenters for COVID-19, an alert for some countries". In: Chaos, Solitons 83 Fractals 139 (2020), p. 110030. DOI: $10.1016 / \mathrm{j}$. chaos . 2020.110030 (cit. on p. 203). 
[62] RM Gray. Entropy and Information Theory. Springer, 1990 (cit. on p. 119).

[63] G. R. Grimmett and D. R. Stirzaker. Probability and Random Processes. New York: Clarendon Press, 2000. ISBN: 0-19-857223 (cit. on pp. 11, 54, $117)$.

[64] G. Guzzetta, P. Manfredi, R. Gasparini, D. Panatto, and W. J. Edmunds. "On the relationship between meningococcal transmission dynamics and disease: remarks on humoral immunity". In: Vaccine 27.25-26 (2009), pp. 34293434. DOI: 10.1016/j.vaccine.2009.01.092 (cit. on p. 164).

[65] M. Hahn and S. Umarov. "Fractional Fokker-Planck-Kolmogorov type equations and their associated stochastic differential equations". In: Fractional Calculus and Applied Analysis 14.1 (2011), 56-79. DOI: 10.2478/s13540011-0005-9 (cit. on p. 4).

[66] Y. Hong, K. L. Hsu, H. Moradkhani, and S. Sorooshian. "Uncertainty quantification of satellite precipitation estimation and Monte Carlo assessment of the error propagation into hydrologic response". In: Water resources research 42.8 (2006). DOI: 10.1029/2005WR004398 (cit. on p. 1).

[67] R. Hovorka et al. "Nonlinear model predictive control of glucose concentration in subjects with type 1 diabetes". In: Physiological Measurement 25.4 (2004), pp. 905-920. DOI: 10.1088/0967-3334/25/4/010 (cit. on p. 209).

[68] W. Hsiu-Chuan. "Mathematical modeling of tumor growth: the MCF-7 breast cancer cell line". In: 16.mbe-16-06-325 (2019), p. 6512. DOI: 10 . 3934/mbe. 2019325 (cit. on pp. 186 sq.).

[69] A. Hussein and M. M. Selim. "Solution of the stochastic radiative transfer equation with Rayleigh scattering using RVT technique". In: Applied Mathematics and Computation 218.13 (2012), pp. 7193-7203. DOI: 10.1016/j . amc.2011.12.088 (cit. on pp. 2, 76). 
[70] National Center for Immunization and Respiratory Diseases. Centers for Disease Control and Prevention. 2017. URL: http: / / www . cdc . gov / meningococcal/index.html (visited on 01/12/2017) (cit. on pp. 162 sq.).

[71] The MathWorks Inc. Particle Swarm Optimization. 2020. URL: https: / / es . mathworks . com / help/gads / particleswarm . html (visited on 07/25/2020) (cit. on p. 192).

[72] Instituto de Salud Carlos III. Protocolos de la red nacional de vigilancia epidemiológica (protocols for the national network for epidemiological surveillance). 2017. URL: http://www . isciii.es/ISCIII/es/contenidos/ $\mathrm{fd}$-servicios-cientifico-tecnicos/fd-vigilancias-alertas/fdprocedimientos/PROTOCOLOS_RENAVE-ciber.pdf (visited on 01/12/2017) (cit. on pp. 162, 165).

[73] L. Jódar and J. C. Cortés. "Rational matrix approximation with a priori error bounds for non-symmetric matrix Riccati equations with analytic coefficients". In: IMA Journal of Numerical Analysis 18.4 (1998), pp. 545561. DOI: 10.1093/imanum/18.4.545 (cit. on p. 103).

[74] J. E. Joy, E. E. Penhoet, and D. B. Petitti. Saving women's Lives: Strategies for Improving Breast Cancer Detection and Diagnosis. National Academies Press (US), 2005. ISBN: 978-0-309-09438-2 (cit. on p. 186).

[75] J. Kapur and J. N. Kesavan. Entropy Optimization Principles with Applications. Academic Press, 1992. ISBN: 978-94-010-5072-2 (cit. on p. 119).

[76] Y. Khan, S. P. Ali-Beik, K. Sayevand, and A. Shayganmanesh. "A numerical scheme for solving differential equations with space and time-fractional coordinate derivatives". In: Quaestiones Mathematicae 38.1 (2015), pp. 4155. DOI: $10.2989 / 16073606.2014 .981699$ (cit. on p. 159).

[77] Y. Khan, M. Fardi, K. Sayevand, and M. Ghasemi. "Solution of nonlinear fractional differential equations using an efficient approach". In: Neural 
Computing and Applications 24 (1 2014), pp. 187-192. DOI: $10.1007 /$ s00521-012-1208-7 (cit. on pp. 3, 159).

[78] Y. Khan, H. Vazquez-Leal, and Q. Wu. "An efficient iterated method for mathematical biology model". In: Neural Computing and Applications 23.3-4 (2013), pp. 677-682. DOI: 10.1007/s00521-012-0952-z (cit. on p. 203).

[79] Y. Khan, Q. Wu, N. Faraz, A. Yildirim, and M. Madani. "New fractional analytical approach via a modified Riemann-Liouville derivative". In: $A p$ plied Mathematics Letters 25 (11 2012), pp. 1729-1733. DOI: 10.1016/j . aml.2012.02.001 (cit. on p. 3).

[80] N. Khemka and C. Jacob. "Exploratory toolkit for evolutionary and swarmbased pptimization". In: The Mathematica Journal 11.3 (2010), pp. 376-391. DOI: $10.3888 / \mathrm{tmj}$.11.3-5 (cit. on pp. 176, 198, 212).

[81] M. Khodabin, K. Maleknejad, M. Rostami, and M. Nouri. "Numerical solution of stochastic differential equations by second order Runge-Kutta methods". In: Mathematical and Computer Modelling 53 (2011), pp. 19101920. DOI: $10.1016 /$ j.mcm. 2011.01 .018 (cit. on p. 3).

[82] A. A. Kilbas, H. M. Srivastava, and J. J. Trujillo. Theory and Applications of Fractional Differential Equations. The Netherlands: Elsevier Science, 2006. ISBN: 978-0444518323 (cit. on p. 3).

[83] P.E. Kloeden and E. Platen. Numerical Solution of Stochastic Differential Equations. Applications of Mathematics. Berlin: Springer, 1992 (cit. on p. 2).

[84] D. P. Kroese, T. Taimre, and Z. I. Botev. Handbook of monte carlo methods. Vol. 706. John Wiley \& Sons, 2013. ISBN: 978-3-642-08107-1 (cit. on p. 177).

[85] S. N. Ladhani, M. Ramsay, R. Borrow, A. Riordan, J. M. Watson, and A. J. Pollard. "Enter B and W: two new meningococcal vaccine programmes 
launched". In: Archives of disease in childhood 101.1 (2016), pp. 91-95. DOI: $10.1136 /$ archdischild-2015-308928 (cit. on p. 164).

[86] C. Li and F. Zeng. Numerical Methods for Fractional Calculus. Vol. 24. CRC Press, 2015. IsBn: 978-1482253801 (cit. on p. 159).

[87] M. Loève. Probability Theory I. Vol. 45. Graduate Texts in Mathematics. New York: Springer-Verlag, 1977. ISBN: 978-1-4684-9464-8 (cit. on pp. 41, $46,50,82)$.

[88] V. Lupulescu and K. N. Ntouyas. "Random fractional differential equations". In: International Electronic Journal of Pure and Applied Mathematics 4.2 (2012), 119-136 (cit. on p. 4).

[89] V. Lupulescu, D. O'Reagan, and G. Rahman. "Existence results for random fractional differential equations". In: Opuscula Mathematica 34.4 (2014), 813-825. DOI: 10.7494/OpMath.2014.34.4.813 (cit. on p. 4).

[90] J. Manimaran, L. Shangerganesh, A. Debbouche, and V. Antonov. "Numerical solutions for time-fractional cancer invasion system with nonlocal diffusion". In: Frontiers in Physics 7 (2019), p. 93. DOI: 10.3389/fphy . 2019.00093 (cit. on p. 186).

[91] J. V. Michalowicz, J. M. Nichols, and F. Bucholtz. Handbook of Differential Entropy. Chapman and Hall/CRC, 2013. ISBN: 9781138374799 (cit. on pp. 118, 120, 186).

[92] K. Mourad and A. Debbouche. "Complete controllability of nonlocal fractional stochastic differential evolution equations with Poisson jumps in Hilbert spaces". In: International Journal of Advances in Applied Mathematics and Mechanics 3.1 (2015), pp. 41-48 (cit. on pp. 2, 4).

[93] M. Mursaleen and S. A. Mohiuddine. Convergence Methods for Double Sequences and Applications. India: Springer, 2014. DOI: 10.1007/978-81322-1611-7 (cit. on p. 15). 
[94] Gianluca N. and Claudio C. "Models of subcutaneous insulin kinetics. A critical review". In: Computer Methods and Programs in Biomedicine 62.3 (2000), pp. 249 -257. ISSN: 0169-2607. DOI: 10.1016/s0169-2607(00) 00071-7. (cit. on p. 209).

[95] T. Neckel and F. Rupp. Random Differential Equations in Scientific Computing. London: Versita, 2013. ISBN: 978-83-7656-025-0 (cit. on p. 3).

[96] K. Nouri and H. Ranjbar. "Mean square convergence of the numerical solution of random differential equations". In: Mediterranean Journal of Mathematics 12 (2015), 1123-1140. DOI: $10.1007 /$ s00009-014-0452-8 (cit. on p. 3).

[97] B. Øksendal. Stochastic Differential Equations: An Introduction with Applications. Berlin-Heidelberg: Springer-Verlag, 2003. ISBN: 978-3540047582 (cit. on p. 2).

[98] S. Oueida, P. A. Char, S. Kadry, and S. Ionescu. "Simulation models for enhancing the health care systems". In: FAIMA Business 85 Management Journal 4.4 (2016), p. 5. DOI: 10.1016/j ·promfg. 2015.07 .155 (cit. on p. 207).

[99] A. Papoulis and S. U. Pillai. Probability, Random Variables and Stochastic Processes. 4th. New York: McGraw-Hill, 2002. ISBN: 0-07-366011-6 (cit. on pp. 50 sq., 171 sq.).

[100] R. K. Pathria and P. Beale. Statistical Mechanics. Academic Press, 2011. ISBN: 9780123821881 (cit. on p. 119).

[101] J. C. Pedjeu and G. S. Ladde. "Stochastic fractional differential equations: Modeling, method and analysis". In: Chaos, Solitons \& Fractals 45.3 (2012), pp. 279-293. DOI: $10.1016 /$ j.chaos.2011.12.009 (cit. on p. 2).

[102] L. Pérez-Breva, R. J. Villanueva, J. Villanueva-Oller, L. Acedo, F. Santonja, J. A. Moraño, R. Abad, J. A. Vázquez, and J. Díez-Domingo. "Optimizing 
strategies for meningococcal C disease vaccination in Valencia (Spain)". In: BMC Infectious Diseases 14.1 (2014), p. 280. DOI: 10.1186/1471-233414-280 (cit. on p. 164).

[103] E. C. Pielou. "An introduction to mathematical ecology". In: (1969) (cit. on pp. 188 sq.).

[104] C. Po-Chen, D. Yimai, M. Vuk, and K. Mladen. "Uncertainty of measurement error in intelligent electronic devices". In: 2014 IEEE PES General Meeting | Conference \& Exposition. IEEE, July 2014. DOI: 10.1109/pesgm. 2014.6938884 (cit. on p. 211).

[105] I. Podlubny. Fractional Differential Equations: An Introduction to Fractional Derivatives, Fractional Differential Equations, to Methods of Their Solution and Some of Their Applications. London: Academic Press, 1998. ISBN: 978-0125588409 (cit. on pp. 164, 169).

[106] T. Prud'homme, A. Bock, G. François, and D. Gillet. "Preclinically assessed optimal control of postprandial glucose excursions for type 1 patients with diabetes". In: 2011 IEEE International Conference on Automation Science and Engineering. 2011, pp. 702-707. DOI: 10.1109/CASE. 2011.6042510 (cit. on pp. 207, 209 sq.).

[107] C. Salas-Eljatib, A. Fuentes-Ramirez, T. G. Gregoire, A. Altamirano, and V. Yaitul. "A study on the effects of unbalanced data when fitting logistic regression models in ecology". In: Ecological Indicators 85 (2018), pp. 502508. DOI: $10.1016 /$ j.ecolind.2017.10.030 (cit. on p. 188).

[108] J. G. Skellam. "Random dispersal in theoretical populations". In: Biometrika 38.1/2 (1951), pp. 196-218. DOI: 10.2307/2332328 (cit. on p. 188).

[109] T. T. Soong. Random Differential Equations in Science and Engineering. New York: Academic Press, 1973. ISBN: 9780080956121 (cit. on pp. 2 sq., 9, 11 sq., 29, 57, 78, 94, 109 sq., 141 sq., 144 sq., 189). 
[110] D. Stanescu, B. Chen-Charpentier, J. J. Brandi, and P. J. S. Colberg. "Random coefficient differential models of growth of anaerobic photosynthetic bacteria". In: Electronic Transactions on Numerical Analysis 34 (2009), pp. $44-58$ (cit. on pp. 104 sq.).

[111] M. F van Stee, S. Krishnan, A. K. Groen, and A. A. de Graaf. "Determination of physiological parameters for endogenous glucose production in individuals using diurnal data". In: BMC Biomedical Engineering 1.1 (2019), p. 29. DOI: $10.1186 /$ s42490-019-0030-z (cit. on p. 207).

[112] C. L. Trotter, N. J. Gay, and W. J. Edmunds. "Dynamic models of meningococcal carriage, disease, and the impact of serogroup $\mathrm{C}$ conjugate vaccination". In: American journal of epidemiology 162.1 (2005), pp. 89-100. DOI: 10.1093/aje/kwi160 (cit. on pp. 164, 178).

[113] V. G. Vaidya and Frank J. Alexandro J. "Evaluation of some mathematical models for tumor growth". In: International journal of bio-medical computing 13.1 (1982), pp. 19-35. DOI: 10.1016/0020-7101(82)90048-4 (cit. on p. 188).

[114] M. T. Valenzuela, G. Moreno, A. Vaquero, M. Seoane, J. C. Hormazabal, M. P. Bertoglia, D. Gallegos, V. Sotomayor, and J. Diaz. "Emergence of W135 meningococcal serogroup in Chile during 2012". In: Revista medica de Chile 141.8 (2013), pp. 959-967. DOI: 10.4067/S0034-98872013000800001 (cit. on pp. 163 sq.).

[115] O. Vallée and M. Soares. Airy Functions and Applications to Physics. London: Imperial College Press, 2010. ISBN: 9781848165489 (cit. on p. 108).

[116] C. F. Van Loan and G. H. Golub. Matrix computations. Johns Hopkins University Press Baltimore, 1983. ISBN: 9780801830105 (cit. on pp. 176, 215). 
[117] V. Varlamov. "Fractional derivatives of products of Airy functions". In: Journal of Mathematical Analysis and Applications 337.1 (2008), pp. 667685. DOI: 10.1016/j.jmaa. 2007.03 .098 (cit. on p. 108).

[118] P. F. Verhulst. "Notice sur la loi que la population suit dans son accroissement". In: Corresp. Math. Phys. 10 (1838), pp. 113-126. DoI: 10.1007/ BF02309004 (cit. on p. 189).

[119] L. Villafuerte, C. A. Braumann, J. C. Cortés, and L. Jódar. "Random differential operational calculus: Theory and applications". In: Computers and Mathematics with Applications 59.1 (2010), pp. 115-125. DOI: 10.1016/ j.camwa.2009.08.061 (cit. on pp. 3, 9).

[120] Roberto Visentin, Michele Schiavon, Rita Basu, Ananda Basu, Chiara Dalla Man, and Claudio Cobelli. "Chapter 6 - Physiological models for artificial pancreas development". In: The Artificial Pancreas. Ed. by R. S. SánchezPeña and Cherñavvsky, D. R. Academic Press, 2019, pp. 123 -152. ISBN: 978-0-12-815655-1. DOI: 10.1016/B978-0-12-815655-1.00015-6 (cit. on p. 207).

[121] T. Weise. Global optimization algorithms-theory and application. 2009. ISBN: 9781611972665 (cit. on p. 196).

[122] M. M. R. Williams. "Polynomial chaos functions and stochastic differential equations". In: Annals of Nuclear Energy 33.9 (2006), pp. 774-785. DOI: 10.1016/j. anucene.2006.04.005 (cit. on p. 2).

[123] A. Worschech, N. Chen, A. Y. Yong, Q. Zhang, Z. Pos, S. Weibel, V. Raab, M. Sabatino, A. Monaco, H. Liu, et al. "Systemic treatment of xenografts with vaccinia virus GLV-1h68 reveals the immunologic facet of oncolytic therapy". In: BMC genomics 10.1 (2009), p. 301. DOI: 10.1186/1471-2164-10-301 (cit. on pp. 190 sq.).

[124] S. B. Yuste and L. Acedo. "An explicit finite difference method and a new von Neumann-type stability analysis for fractional diffusion equations". 
In: SIAM Journal of Numerical Analysis 42.5 (2005), pp. 1862-1874. DOI: 10.1137/030602666 (cit. on p. 164).

[125] S. B. Yuste, L. Acedo, and K. Lindenberg. "Reaction front in an A+ B $\rightarrow$ C reaction-subdiffusion process". In: Physical Review E 69.3 (2004), p. 036126. DOI: 10.1103/PhysRevE.69.036126 (cit. on pp. 164, 169).

[126] Y. Zhu. "Uncertain fractional differential equations and an interest rate model". In: Mathematical Methods in the Applied Sciences 38.15 (2015), pp. 3359-3368. DOI: 10.1002/mma.3335 (cit. on p. 2). 
Uncertainty quantification collects different methods and computational techniques aimed at describing the randomness in real phenomena. These methods are useful in the modelling of different processes as biological, physical, natural or social, since they present some aspects that can not be determined exactly. They may not always be fully described and therefore involve uncertainties that affects on the final result. The main objective of this $\mathrm{PhD}$ thesis is to extend techniques to quantify the uncertainty in two mathematical areas: fractional calculus and mathematical modelling.

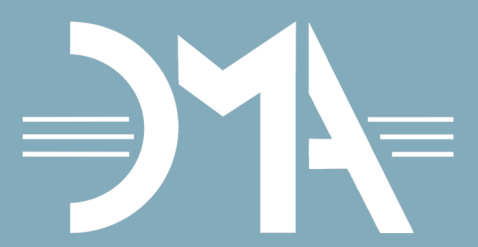

DEPARTAMENT

DE MATEMÀTICA

APLICADA 